
eHealth vs. Infection

Participatory development of persuasive eHealth to support safe care

Nienke Beerlage-de Jong 
Thesis, University of Twente, 2016

(C) Nienke Beerlage-de Jong, Enschede, the Netherlands ISBN: 978-90-365-4199-2

Cover design by Buro Bam!

Printed by Gildeprint, the Netherlands 


\title{
EHEALTH vs. INFECTION \\ PARTICIPATORY DEVELOPMENT OF PERSUASIVE EHEALTH TO SUPPORT SAFE CARE
}

\author{
PROEFSCHRIFT
}

\author{
Ter verkriiging van \\ de graad van doctor aan de Universiteit Twente, \\ op gezag van de rector magnificus, \\ prof. dr. H. Brinksma, \\ volgens besluit van het College voor Promoties \\ in het openbaar te verdedigen \\ op donderdag 24 november 2016 om 12.45 uur

$$
\text { door }
$$ \\ Nienke Beerlage-de Jong \\ geboren op 20 december 1986 \\ te Oldenzaal
}


Dit proefschrift is goedgekeurd door de promotor Prof. dr. J.E.W.C. van Gemert-Pijnen en copromotoren Prof. dr. R. Sanderman en Prof. dr. A.W. Friedrich. 
Samenstelling promotiecommissie

Promotor Prof. dr. J.E.W.C. van Gemert-Pijnen

University of Twente, University Medical Centre Groningen, University of Groningen

Co-promotoren Prof. dr. R. Sanderman

University Medical Centre Groningen, University of Groningen,

University of Twente

Prof. dr. A.W. Friedrich

University Medical Centre Groningen, University of Groningen

Leden

Dr. C.J.M. Doggen

University of Twente

Dr. R.M.G. Hendrix

Certe, University Medical Centre Groningen, University of Groningen

Prof. dr. H. Hermens

University of Twente, Roessingh Research and Development

Prof. dr. H. Oinas-Kukkonen

University of Oulu

Prof. dr. J. Scheres

Maastricht University Medical Centre

Prof. dr. B.N.M. Sinha

University Medical Centre Groningen, University of Groningen 



\section{Contents}

Chapter $1 \quad$ General introduction 9

Part 1 Participatory development 29

Chapter 2 Opening the black box of Clinical Decision Support 33

Systems for antimicrobial stewardship - A scoping review

Chapter 3 The importance of Participatory Development for 59

antimicrobial stewardship

\section{Part 2 Persuasive design}

Chapter 4 Practical guideline for developing eHealth technology for healthcare professionals, by combining UCD with the PSD model

\section{Part 3}

Measuring persuasiveness

Chapter 5 Combining user-tests, log-data and expert-assessments to evaluate constructs of the Perceived Persuasiveness Questionnaire

Chapter 6 Evaluation of the Perceived Persuasiveness Questionnaire

Part 4

Reflecting on our work

Chapter 7 Technology to support integrated Antimicrobial Stewardship

Programs; A user-centered and stakeholder driven

development approach

Chapter 8 General discussion

Samenvatting (Summary in Dutch)

Dankwoord (Acknowledgements in Dutch)

Publications and other output 



\section{General introduction}


Before you start reading this thesis, may I ask you to do one thing? Imagine a wise scientist thinking about how the world can be just a little safer for patients. Imagine this scientist trying to devise a way to prevent infections from passing to patients and to avoid antimicrobial resistance.

Now allow me to guess what the picture in your head looks like. First of all (unless you are emancipated or work at the Center for eHealth and Wellbeing Research in The Netherlands), you probably pictured a man with slightly grey hair. Maybe you pictured this man sitting behind a stack of thick medical books piled upon his desk?

Many observations could be ascertained about this picture. But let's focus on the desk. Why is he sitting there? Why is he not walking around, talking to the people in the field that he wants to improve? Why isn't he examining the conditions under which they perform their work?

This thesis is about how scientists (of any age or gender) can step out from behind their desk and enter the world of clinical practice. In the field that we focus on - antimicrobial stewardship and infection management - a gap exists between protocols and guidelines (written by experts) on the one hand and clinical practice on the other. We think we can bridge this gap through our approach to eHealth. Within this thesis, eHealth is used to bring the two worlds of experts and clinical practice together. Why? One of the participants (a member of the infection committee of a Dutch hospital) in our research clearly explains it for you.

"When we check the protocols, we just read them through and find all the necessary information. We don't think to do it the other way around, starting with the problem, like you are doing now. And how easy is it to find the information that you need then! Because, I really know these protocols, probably more than others since I am a member of our hospital's Infection Committee. Even still, if I look at them from this new perspective, I have to conclude that some things are really inconvenient."

[Infectieziekten Bulletin, 2013, 24(8), p. 259]

The quote above describes the essence of what this thesis is about. It is illustrative of the differences in approaching protocols and guidelines between experts and clinical practice. So from one perspective, this could have been the shortest thesis ever.

Yet, having come this far, we will seize the opportunity to explain the logistics behind the research upon which this thesis is based. After all, the development of eHealth technology is not pursued for the sole purpose of developing eHealth as such. Rather, in promoting eHealth technology, we aim to contribute to modern healthcare by supporting safe care. 


\section{About this thesis}

Within the first chapter of this thesis, we introduce the foremost threat to patient safety throughout the world: the problem of antimicrobial resistance [3]. We describe how this problem is currently dealt with through the application of Antimicrobial Stewardship Programs (ASPs). Next, we describe the challenges of providing successful ASPs, as suboptimal use of and adherence to its interventions can prevent the best application of ASPs. We elaborate on why we feel eHealth can play a role in increasing ASPs' success, specifically in supporting physicians, who are key stakeholders in ASPs. We believe participatory development of persuasive eHealth technology (applied via the CeHRes Roadmap) is pivotal to supporting the use of, adherence to and success of eHealth to support physicians in ASPs. This viewpoint is further supported by our research as described in Chapters 2 to 7.

In Chapter 2, we show that the current technology that supports ASPs is expert- or guideline-driven, as illustrated by Clinical Decision Support Systems (CDSSs). Physicians (end-users) were barely involved in the development of these CDSSs. In Chapter 3, we provide insights into how the participation of physicians (and other stakeholders) can shape the development of CDSSs for ASPs and the added value of doing so.

Besides the contribution stakeholders can provide in the development of such programs, referred to as the participatory development process of technology, in Chapter 4, we demonstrate that the application of persuasive elements within a technology can offer users support and motivation during its use. In particular, we show how participatory development and persuasive systems design can be combined during the development of a web-based registration and monitoring system for nursing home infections.

In addition to developing and designing persuasive eHealth technology to support physicians in ASPs, the evaluation of the success of eHealth technology is, of course, pivotal to be able to tell whether this was done successfully. Therefore, Chapters 5 and 6 focus on the evaluation of persuasive eHealth technology. These chapters concentrate on the preliminary stages of establishing a validation of the Perceived Persuasiveness Questionnaire, which measures whether technology users experience persuasive elements.

Finally, in Chapter 7, we provide an overview of the lessons learned from the several technologies that we developed to support ASPs. In particular, we provide understanding of how the CeHRes Roadmap was applied to the development and implementation of these technologies and what kind of knowledge this Roadmap yielded.

Let us now turn to the problem that underpins this thesis: antimicrobial resistance. This is one of the major threats to patient safety in modern healthcare. After describing why it is such a threat to safe care, we describe how eHealth can contribute to dealing with the presented problem. 


\section{Safe care}

Offering high-quality healthcare requires healthcare to be safe, not only for the people working in the field of healthcare, but also for their patients. Patients place their faith in the safety of the healthcare system and the people working to take care of them. The extensively cited book To Err is Human describes how patients can be in harm's way just because of the fact that they require and make use of healthcare [7]. Patient safety is and always has been of paramount importance for high-quality healthcare. It is defined as the "discipline in the health care sector that applies safety science methods toward the goal of achieving a trustworthy system of health care delivery. Patient safety is also an attribute of health care systems; it minimizes the incidence and impact of, and maximizes recovery from, adverse events" [8]. Standards of what good patient safety is, and the definition of high-quality healthcare have progressed with the development of modern medicine. Never before have clinicians been able to treat so many illnesses. Never before have so many terminal illnesses been turned into chronic ones. These recent developments are, of course, very positive. Nevertheless, they present healthcare and society with new challenges, in particular antimicrobial resistance [9].

Many of the advances in treatment possibilities have been enabled by antimicrobials. Their important discovery, in the 1920's, might very well have provided the highest impact, revolutionizing modern healthcare. Many once fatal illnesses can now be treated or even cured thanks to antimicrobials [10]. However, with this significant progress in healthcare also came new challenges. Nowadays, the misuse of antimicrobials is the number one threat to patient safety, as this misuse can lead to Healthcare-Associated Infections (HAls).

\subsection{The significance of Antimicrobial Stewardship Programs [ASPs]}

Despite the seemingly endless possibilities of antimicrobials, they remain definitely limited. To be precise, every time an antimicrobial is used, pathogens become slightly more resistant against the antimicrobial [1 1]. If this trend is not slowed down or changed, antimicrobials might ultimately lose their overall effectiveness [12, 13], making infections that are now common and treatable, once again life threatening. Therefore, the World Health Organization recognizes antimicrobial resistance as one of the major threats for not only patient safety, but even for human health as a whole [3].

With the rise of resistant pathogens, the number of Healthcare-Associated Infections (HAls) also increases. These are infections that people acquire merely because they are hospitalized or admitted to any kind of healthcare institution. In fact, the Centers for Disease Control and Prevention stated that in the United States of America, "on any given day, one in every 25 hospital patients has at least one healthcare-associated infection" [14]. In Europe, this number is one in every 18 hospital patients [15]. Besides the fact that these infections are difficult to treat, they also tend to (rapidly) spread from one 
patient to the next due to the density of populations in healthcare settings. Even friends or family members visiting a patient can 'carry' a pathogen with them and infect patients.

Due to the extreme complexity of this challenge, dealing with the threat of antimicrobial resistance requires a multimodal approach, referred to as an Antimicrobial Stewardship Program (ASP) [16]. Within such a program, multiple interventions are (preferably) combined to ensure that the right antibiotic, in the right dosage, for the right duration is used to treat a patient [9]. An intervention within an ASP can, for example, use protocols and guidelines for the prescribing of antimicrobials or the implementation of technology that provides informational support to healthcare professionals [17, 18]. However, the implementation of such interventions remains challenging $[4,19]$.

The development and implementation of ASPs are medically based and clinically focused, which appears logical. After all, these ASPs serve a clinical purpose, they operate in a clinical setting, and are aimed at helping clinicians with their daily clinical tasks. However, a singular clinical focus actually results in ASP interventions that are available, but not frequently used nor adhered to. A clinical focus has been shown to contribute to a poor fit between ASP interventions on the one hand and healthcare professionals' needs and clinical practice on the other. Such a myopic vision neglects the behavioral change that is often required from healthcare professionals if they want to adhere to an ASP intervention [6]. Additionally, the attitudes and perceptions of physicians and nurses towards the severity of antimicrobial resistance can greatly contribute to - or hinder - the ASP's success. Strikingly, several studies have shown that both physicians and nurses believe that the problems of antimicrobial resistance are more urgent on a national level than within their own institution [20, 21]. Additionally, there is a lack of incentives to adhere to ASPs, and the negative effects of antimicrobial misuse are not visible to or experienced by the individuals responsible for implementing the ASP [22]. Such attitudes and perceptions may contribute to a low feeling of urgency and a lack of problem ownership, hindering the adoption of ASP interventions [23].

Undoubtedly, a clinical focus is required to create and validate the content of ASPs. However, the importance of behavioral and social sciences for the successful implementation of ASPs is recently being recognized and acknowledged [24-26]. In fact, the limited use of and adherence to such interventions is a highly complex behavioral, perception and attitude problem. ASPs require healthcare professionals to adjust their own (safety) behavior to mainly protect the health of others (i.e., patients), but also their own health. To do so, they must become aware of their own role within the chain of events that cause antimicrobial resistance. Problems of this nature have been classified as 'wicked,' meaning that the exact cause of the problem is unclear, that many stakeholders are involved, and that no clear end-point can be reached by finding a solution [27].

We strongly believe that technology offers a great opportunity to deal with the behavior, perceptions and attitudes that enable a 'wicked' problem such as antimicrobial resistance 
to persist. For example, the use of technology offers a means to easily communicate and share information [4]. Technology also contributes to creating a better fit between ASP interventions and clinical practice $[4,6]$. To be able to design the most fitting solutions, the development of such technologies must be synthesized with a behavioral or social approach. Or rather: it requires a socio-technical approach. Such an approach focuses on the interplay between technology and its social and technical context, such as existing work processes, technologies and social interactions [28].

This current thesis builds upon three prior theses that have examined the problem of antimicrobial resistance and infection management from different perspectives. Each study focused on using technology (I) to bridge the gap between the end-users who have to work with these interventions in daily clinical practice and the experts who develop the interventions and/or guidelines [4], (II) to overcome the gap between the intervention and the context within which it is intended to be used [6], or (III) to increase the 'implementability' of the interventions for sustainable use [5]. The first thesis adopted the perspective of protocols and guidelines [4], the second thesis focused on the nurses' perspective [6] and the third applied an organizational perspective [5].

As a continuation of these three theses and their line of research (see also Box 1), this thesis zooms in on the perspective of physicians and how they can be supported in performing their tasks in ASPs. From the earlier theses, we have learned that it is essential to cooperate with end-users and other stakeholders during the development of eHealth technology for ASPs and that it is of paramount importance that a technology is pleasant to work with and motivates its users [4-6]. Within the current thesis, we aim to provide insights into why this is the case, how this knowledge can be applied to support physicians in performing their tasks in ASPs and the lessons learned that might be applicable to future development processes. We describe what a socio-technical approach to eHealth development entails, what kind of knowledge it can yield, and how these results can be evaluated. To begin, we first elaborate on our perspective on what eHealth is and how it can contribute to ASPs. 
Box 1. Crossing borders in antimicrobial stewardship and infection management

As cross-border traffic of people, livestock and patients increases, pathogens are crossing borders as well [2]. No matter how stringent a nation's border control, resistant pathogens are obviously able to pass undetected through customs. In addition to crossing national borders, antimicrobial stewardship and infection management must cross institutional borders as well. Patients often travel from hospital to nursing home or revalidation centers (and vice versa), and pathogens travel with them. And last but not least, pathogens cross disciplinary borders. To ensure successful antimicrobial stewardship and infection prevention and management, all kinds of healthcare providers (e.g., nurses, clinicians and laboratory technicians) and other stakeholders (e.g., patients, hospital administration and technology developers) are required to closely cooperate to guard against pathogens spreading.

In 2005, the EUREGIO MRSA-net Twente/Münsterland was launched as a platform to enable such cross-border cooperation. It was initiated to create a network of healthcare providers who could cooperate in the surveillance, prevention and management of infections caused by MRSA (Methicillin Resistant Staphylococcus aureus) [2]. This cooperation is enabled and supported through the use of technology [4].

The work initiated in the MRSA-net project continued on a broader scale in the INTERREG IVa EurSafety Health-net project. This project also strives towards creating a strong network of healthcare providers and other stakeholders to increase patient safety in cross-border healthcare. No longer specifically aimed at MRSA, their work includes all Healthcare-Associated Infections. The University of Twente is involved in the projects by providing (eHealth) technology to support its aims $[5,6]$. The research in this thesis was also performed in the context of the EurSafety Health-net project.

\section{2. eHealth technology to support safe care}

As stated in Box 1, the University of Twente is involved in the fight against antimicrobial resistant pathogens through the development of supportive technology. Such technology is referred to as "eHealth," which refers to the use of Information and Communication Technology (ICT) to enhance health(care) and wellbeing. In our view, eHealth technology is a very broad term, perhaps best captured by the statement of Eysenbach [29], who in 2001 wrote:

"(...) the term characterizes not only a technical development, but also a state-of-mind, a way of thinking, an attitude, and a commitment for networked, global thinking, to improve healthcare locally, regionally, and worldwide by using information and communication technology" (p. e20) 
In line with this broad definition of eHealth, many different types of eHealth technology are available, ranging from relatively straightforward informative websites to highly complex systems. Today eHealth exists to improve mental health and wellbeing [30, 31$]$ and support healthcare [4, 6], incorporating web-based systems that are solely focused on PC use, as well as responsive technology that can be used on an individual's PC, tablet, smartphone, or even accessed through Virtual Reality [32, 33].

Despite the broad range of possibilities of eHealth and its (widely acknowledged) added value for safe care, eHealth technology often does not succeed in reaching its full potential [34, 35]. Problems with the successful application of eHealth are not new, having always co-existed within the field of eHealth technology [36]. However, in addition to its unquestionable potential, eHealth will become more of a necessity in the near future to ensure high-quality healthcare because of the increase in the aging population and the growing number of treatment possibilities provided by modern medicine [10].

Perhaps one of the paradoxes of eHealth technology is that, despite its potential and the seemingly endless variety of ways it can be developed, implemented and evaluated, a 'golden standard' or recipe for eHealth success is difficult to find [34]. Nevertheless, some prerequisites are available. It is known that key to the development of successful and sustainable eHealth technology is to involve stakeholders in every stage from problem definition to the technology's evaluation [6, 34, 35]. Consequently, this also holds true for eHealth that is designed to support physicians in ASPs since working with a technology affects their work and work processes [4, 6]. Additionally, the sustained use of and adherence to such technology is a well-known problem for eHealth in general $[37,38]$ and for ASP specifically [39, 40]. Previous studies have shown that applying persuasion within technologies to support and motivate users during their use of the technology is beneficial for its success [6].

One approach that combines participatory development and persuasive design is the CeHRes Roadmap, developed by the Centre for eHealth and Wellbeing Research of the University of Twente [35]. This Roadmap and its implications for eHealth technology are described in the next section. By applying the Roadmap to deal with the challenges for eHealth technology in ASPs, (from a scientific point of view) we expand the Roadmap with knowledge on: how a participatory development process of persuasive eHealth technology to support physicians in an ASP can be shaped, what kind of information it yields, and how its effects can be evaluated. From a practical point of view, by applying such an approach, within this project, eHealth technology was developed to fit the working- and thinking-processes of its users. All this research was conducted in order to enable and support safe care via eHealth technology. 


\section{Our approach for the development of eHealth technology}

This section describes our approach towards the development of a successful eHealth technology, focusing on the CeHRes Roadmap in general, and participatory development and persuasive design in particular.

\subsection{The CeHRes Roadmap}

The Center for eHealth and Wellbeing Research of the University of Twente has developed a framework - a Roadmap - for the holistic participatory development of eHealth technology [35]. Drawing from participatory development, persuasive technology and business modeling, the Roadmap is based upon six main principles. It assumes that eHealth development: (I) is a participatory process, (II) involves continuous (formative) evaluation cycles, (III) is intertwined with implementation, (IV) changes the organization of healthcare, $(\mathrm{V})$ should involve persuasive design techniques, and (VI) needs advanced methods to assess impact.

The Roadmap guides eHealth technology developers, researchers and policy makers through the processes of problem definition to successful eHealth technology. By applying participatory development principles throughout all stages of the Roadmap, it contributes to achieving a good fit between technology and its use context. Persuasive design (mainly reflected in the Design stage of the Roadmap) can enable and motivate potential endusers to actually use the technology. Through business modeling, which is used throughout the Roadmap with a focus on successful Operationalization, the Roadmap strives towards the development of sustainable eHealth technology. As mentioned before, this thesis focuses on the first two underlying elements of the Roadmap: participatory development (see Section 3.1.1) and persuasive design (see Section 3.1.2).

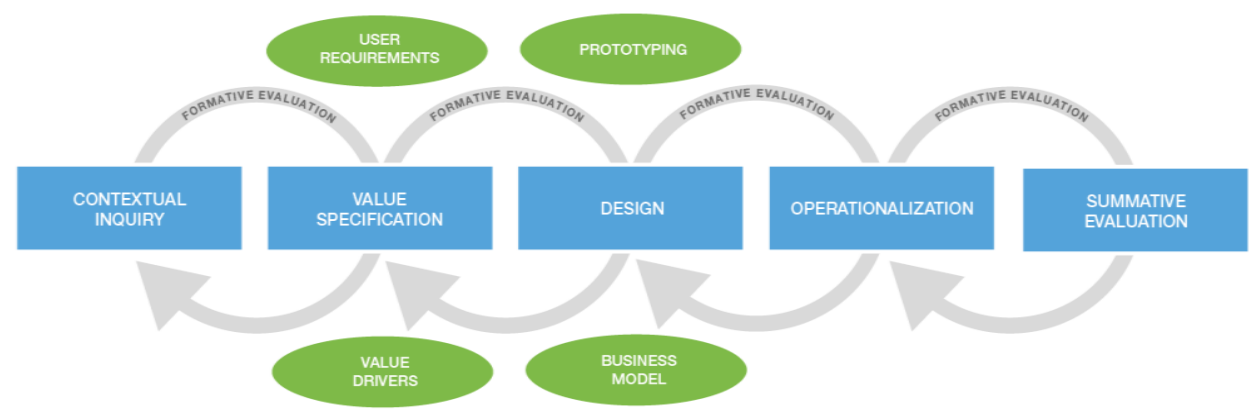

Figure 1. The CeHRes Roadmap [35]

\subsubsection{Participatory development}

In their study into the factors affecting the long-term acceptance and use of an eHealth technology, Nijland et al. [41] found that applying participatory development was one of the keys to success. Throughout the years, participatory (IT) development has been 
defined and applied in different ways, varying in both the degree of participation and in whom is invited to participate. However, it is widely accepted that participation of stakeholders in the development process is crucial for eHealth success [42]. Therefore, participatory development is one of the pillars upon which the CeHRes Roadmap is based.

While Kukafka et al. [43] promote participatory design via a framework that allows for the effective engagement of 'system end-users' from the start of development, in our view, participatory development can and should, in fact, reach beyond involving system end-users. Other stakeholders, such as policy makers, IT developers, and management, can also provide essential input. This approach is also supported by Bødker et al. [44], who state that 'only' involving potential end-users of a technology creates a risk that the technology will be impaired from 'inside reasoning'. Potential end-users of a technology all usually have some traits in common; they belong to a particular healthcare profession [17], suffer from a certain disease [45], or their lives are affected by a similar event [30]. By solely relying on their input for the development of technology, their perspectives and visions may become overbearing. For example, the Nurse Antibiotic Information app is a technology that supports a particular healthcare profession, that is, hospital nurses. The technology has shown to successfully support nurses in performing their tasks and in communicating with physicians in ASPs [46]. However, nurses might not have even been recognized as (key) stakeholders for ASPs without the application of participatory development that started with an elaborate stakeholder analysis, in which not only nurses but also other (key) stakeholders participated (e.g., physicians, pharmacists, clinical microbiologists and technology developers) [47].

Regarding the degree of involvement of stakeholders, Bødker et al. [44] suggest that 'insider reasoning' can be prevented by informing other stakeholders about the technology's goals, visions and plans during development. They refer to this as 'anchoring visions' [44], allowing stakeholders to become accustomed to organizational changes that may be required to work with the technology and to prevent unrealistic expectations. However, participatory development should entail more than 'informing' stakeholders. They should play an active role during every stage of development - from problem definition and stakeholder analysis to the implementation and evaluation of the technology. This active role can be shaped by such tasks as being involved in formulating the problem statement, providing stakeholder analysis, and testing and suggesting improvements to technology prototypes. But such participatory development may also extend as far as actively involving end-users and other stakeholders in planning, managing and executing the entire developmental process [44].

Finally, especially in the field of infection control and antimicrobial stewardship, another kind of stakeholder should not be overlooked. For decades, protocols and guidelines have been the cornerstones of providing evidence-based care [48], allowing physicians to prescribe antimicrobials according to state-of-the-art scientific evidence. Thus, 
protocols and guidelines should (as far as they are available) in our view also be 'participating' in the development. Consequently, protocols should always be considered, ensuring that the technology used in the clinical practice does not contradict them, thus causing unnecessary confusion and frustration among healthcare professionals.

All in all, the development of eHealth technologies that involve end-users and other stakeholders in every stage of their process and carefully consider relevant protocols and guidelines are more likely to succeed than expert- or guideline-based technologies. In addition to participatory development in which technology designers structurally cooperate with stakeholders, persuasive design can also help achieve successful use of and adherence to eHealth amongst end-users [35]. The next section describes the background and possibilities of this aspect of the CeHRes Roadmap.

\subsubsection{Persuasive eHealth technology}

Persuasive technology is a way to design eHealth technology in order to change its users' behavior and/or attitudes [49] without using coercion or deceiving the technology's users [1]. In our view, it is paramount that users of eHealth are continuously motivated and engaged through the technology itself. Persuasion can take place through both humancomputer communication (when a message travels from a computer to a human, or vice versa) and computer-mediated communication (when a message travels between humans, through a computer) [1, 37]. The latter places more emphasis on the communicative character, rather than its informative character, of the technology [37], implying that the way the technology itself 'interacts' with the user has an effect on how willing the users are to 'interact' with it. That being said, to stimulate a pleasant communication, and thus also sustainable use of eHealth technology, the Persuasive Systems Design (PSD) model offers a framework for applying and evaluating persuasive design elements [1]. This model is mainly incorporated in the Design stage of the CeHRes Roadmap [35].

\section{The Persuasive Systems Design model}

The PSD model builds upon earlier research by Fogg [49]. Based on and complementing Fogg's work, Oinas-Kukkonen \& Harjumaa provide a framework to concretize and apply the theoretical concept of persuasive technology [1] by suggesting a stepwise approach to studying the intended 'persuadees' (i.e., the end-user) and their persuasion context. Based on their framework, certain techniques can be selected and applied in the Design phase of the CeHRes Roadmap and hence be incorporated into the actual design of the technology's persuasive content and functionality [1]. These techniques are divided into four categories: Primary Task Support, Dialogue Support, Social Support and System Credibility Support (see Figure 2). The category of Primary Task Support is described as the means the technology offers to aid its user in performing his or her primary task [1]. This support is for example provided via Tunneling, which has the technology guide the user through a prescribed process. Dialogue Support is the interaction between the technology and its users that aims to keep the user active and motivated [1]. An example 
of a design technique through which this support is offered is Suggestion, wherein the technology offers suggestions that fit the specific characteristics of an individual user [1]. Social Support allows the user to be supported by and part of a social network via the technology. An example of such support is when the technology offers the possibility to compete with other users of the technology toward specific goals (referred to as 'Competition') [1]. Finally, System Credibility Support is the believability of the technology, based on the user's first impression (subjective component) and by providing respected and renowned sources (objective component). This technique is, for example, achieved by making certain the technology looks and feels competent to its users [1].

To date, studies have yet to determine whether all of these design principles should be combined and incorporated into a technology for it to be persuasive, nor is it known what elements work best for whom and in what situation [38,50], or whether it works best if users are consciously aware of the persuasive design elements. Thus, the PSD model not only provides great opportunities for the development of successful eHealth technology, it also presents us with many interesting questions to investigate and answer.

\section{Persuasive Systems Design model}

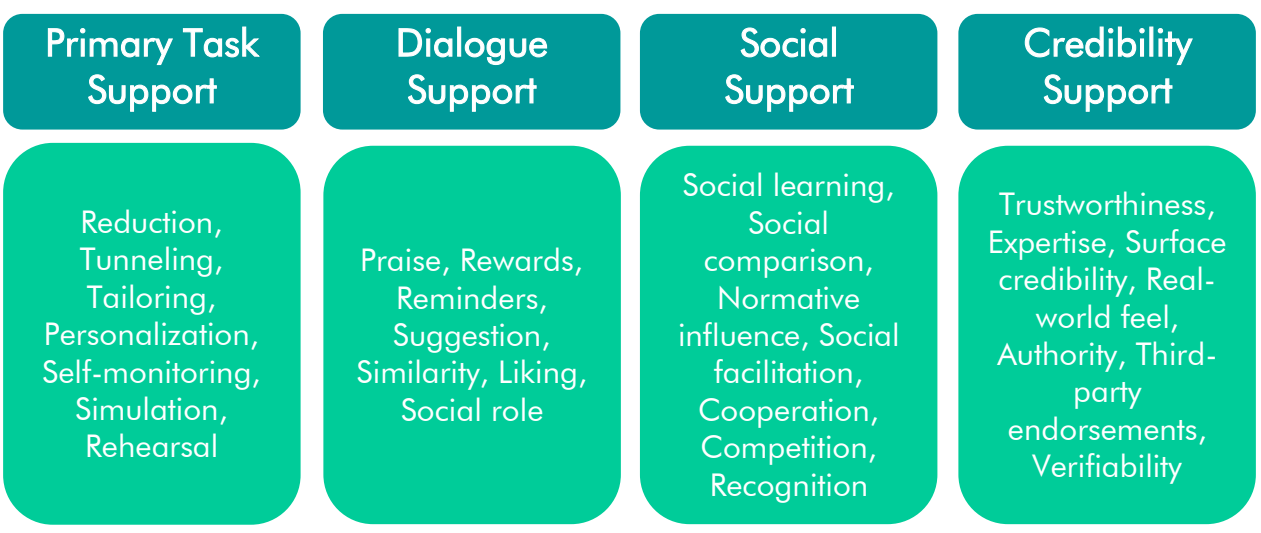

Figure 2. The Persuasive Systems Design model (based on [1])

\subsubsection{Evaluation of persuasive eHealth technology}

By applying the CeHRes Roadmap, and particularly the principles of participatory development and persuasive design, eHealth technology can be developed that is intended to optimally support healthcare professionals in ASPs. However, to what extent the eHealth technologies are actually supportive, usable and user friendly can only be determined through a thorough evaluation. Moreover, evaluation of such technologies can provide insights into the (to date largely unknown) working mechanisms of persuasion and the factors that contribute to sustained use of and adherence to eHealth technology. Very few methods for such evaluations are available, although recently the Perceived Persuasiveness Questionnaire (PPQ) [51] based on the PSD model [1] has been formulated to evaluate whether the users of a technology actually experience its 
persuasive elements. The PPQ contains a total of 31 questions about the different aspects of the PSD constructs, complemented with Perceived Effectiveness, Perceived Effort, Perceived Unobtrusiveness, Perceived Persuasiveness and Use Continuance. The PPQ has already been applied in several studies to evaluate various eHealth technologies [52, 17]. However, the PPQ has not been validated yet, making it impossible to compare scores between technologies. Moreover, no research to date has been conducted on whether the questions actually measure the constructs they intent to measure (construct validity). Despite these unknown factors, the PPQ offers a great opportunity for gaining insights into the persuasive mechanisms within eHealth technology and explore its possibilities.

\section{Research questions}

These previously described frameworks and models form the basis of the research within this thesis, which further focuses on how such frameworks and models can be used to support physicians in ASPs. This thesis supplements earlier research that focused on protocols and guidelines [4], nurses [6] and an organizational perspective [5], by adding the perspective of physicians. Thus, the main research question of this thesis is:

How can eHealth technology stimulate safe care by supporting physicians in Antimicrobial Stewardship Programs?

Specifically, the current thesis focuses on how participatory development and persuasive design can answer this question. Therefore, the main research question is answered through several sub-questions that are elaborated upon in the chapters of this thesis:

- How can a participatory development process of eHealth technology contribute to supporting physicians in ASPs? (Chapters 2 and 3)

- How can persuasive design of eHealth technology contribute to supporting physicians in ASPs? (Chapter 4)

- How can the success of persuasive eHealth technology be evaluated? (Chapters 5 and 6)

- What can we learn from applying the CeHRes Roadmap in the development and implementation of technology to support ASPs? (Chapter 7) 


\section{Conclusions and thesis outline}

Within this chapter, we introduced the most pressing threat to patient safety in modern healthcare - antimicrobial resistance and Healthcare-Associated Infections. Dealing with this threat requires us to take action to prevent such infections from occurring (for example, via good hygiene) and to diminish the rise of antimicrobial resistance (for example, via prudently prescribing antimicrobials). ASPs bundle the efforts that are undertaken in this context, and we propose to make use of the possibilities of eHealth technologies to support such programs. Earlier research has already rendered insights into some of the possibilities for providing such support. The current thesis specifically aims at using eHealth technology to support physicians in their ASP tasks, such as registering and monitoring infections and prudently prescribing antimicrobials. We do so, using two frames of reference in the development of such technology: participatory development and persuasive design.

The aim of this thesis is to provide insight into how participatory development and persuasive design can contribute to supporting physicians in ASPs who use eHealth technology, why it works and how its effects can be evaluated. This thesis is divided into four parts, each focusing on a different aspect that is relevant to studying the core tasks of physicians in ASPs.

The first part is focused on Clinical Decision Support Systems (CDSSs) that support physicians to prudently prescribe antimicrobials. Chapter 2 concerns a scoping literature review, aiming to look into the research to date regarding how CDSSs are currently being developed. Part 1 demonstrates that despite the fact that prior research has proven the importance of participatory development, end-users and other stakeholders are hardly involved in current development. Chapter 3 further elaborates on this finding. Via prototype evaluations and an online questionnaire, we demonstrate why it is so important to involve end-users and other stakeholders in the development process of eHealth technology to support physicians in an ASP (specifically in the case of CDSSs).

The second part of this thesis is based on the case of a web-based registration and monitoring system for HAls in nursing homes. Chapter 4 provides an overview of the development process of this technology, wherein participatory development and persuasive design were combined to achieve an optimal fit between the technology, its users and context. This process was achieved via an expert discussion and online questionnaire, in depth-interviews and scenario-based user-tests with potential endusers. The findings demonstrate how the combination of participatory development and persuasive design can be applied and how it can provide an understanding of and the means to deal with the needs and wishes of the end-users.

Part 3 explains the development of an evaluation method for the persuasiveness of (eHealth) technology, via the Perceived Persuasiveness Questionnaire (PPQ). Chapter 5 presents a pilot study that is the first to assess the possibilities of the PPQ by retrospectively 
applying the questionnaire to a technology (described elsewhere [17]) that supports nurses in ASP. We analyzed the match between expert-assessments of the presence of persuasive elements within the technology and user-tests with potential end-users, and results were promising. Chapter 6 describes the efforts that were then undertaken to progress towards the validation of the PPQ. Subjective evaluations of a technology (the RunKeeper app) were combined with a closed-ended card sort study to evaluate the construct validity of the PPQ. This study has identified the strengths and some of the limitations of the PPQ and has shown opportunities for future work that is needed in order to obtain a validated method for the evaluation of perceived persuasiveness.

Part 4 consists of reflections on the work that was performed in this and former [4-6] theses. Chapter 7 provides an overview of several technologies that were developed by our research group to support ASPs. Specifically, in this chapter, the developed technologies illustrate how the different stages of the CeHRes Roadmap were and can be applied. We provide practical suggestions for the development of technology to support ASPs, but also demonstrate what kind of information it yields and how it can contribute to the success of ASPs. Finally, Chapter 8 provides the General Discussion of the research performed in this thesis, reflecting on its main findings and describing the implications for successful ASP technology. In addition, the strengths and limitations of the research are discussed, suggestions for future research are offered and a final conclusion is drawn. 


\section{References}

1. Oinas-Kukkonen, H. and Harjumaa, M., Persuasive systems design: Key issues, process model, and system features. Communications of the Association for Information Systems, 2009, 24(1), p. 28.

2. Friedrich, A., Daniels-Haardt, I., Kock, R., et al., EUREGIO MRSA-net Twente/Munsterland-a Dutch-German cross-border network for the prevention and control of infections caused by methicillin-resistant Staphylococcus aureus.

Eurosurveillance, 2008, 13(35), p. 1-5.

3. World Health Organization, Antimicrobial resistance global report on surveillance: 2014 summary. 2014.

4. Verhoeven, F., When staff handle staph: user-driven versus expert-driven communication of infection control guidelines, 2009, University of Twente: Enschede.

5. Limburg, A.H.M., Implementing antibiotic stewardship: involving stakeholders in eHealth, 2016, University of Twente: Enschede.

6. Wentzel, M.J., Keeping an eye on the context: participatory development of eHealth to support clinical practice, 2015, Universiteit Twente: Enschede.

7. Kohn, L.T., Corrigan, J.M., and Donaldson, M.S., To err is human: building a safer health system. 2000: National Academies Press.

8. Emanuel, L., Berwick, D., Conway, J., et al., What exactly is patient safety? In: Hendriksen, K., Battles, J.B., Keyes et al. eds. Advances in patient safety: new directions and alternative approaches, Rockville: Agency for Healthcare Research and Quality, August 2008.

9. Pollack, L.A. and Srinivasan, A., Core elements of hospital antibiotic stewardship programs from the Centers for Disease Control and Prevention. Clinical Infectious Diseases, 2014, 59(suppl 3), p. S97-S100.

10. Clardy, J., Fischbach, M.A., and Currie, C.R., The natural history of antibiotics. Current Biology, 2009, 19(11), p. R437-R441.

11. MacDougall, C. and Polk, R.E., Antimicrobial stewardship programs in health care systems. Clinical Microbiology Reviews, 2005, 18(4), p. 638-656.

12. Alanis, A.J., Resistance to antibiotics: are we in the post-antibiotic era? Archives of Medical Research, 2005, 36(6), p. 697-705.

13. Wentzel, J., Limburg, M., Karreman, J., et al. Participatory Development of a 2.0 Platform to Implement an International Antibiotic Stewardship Program. In: Medicine 2.0 Conference, 2011, Stanford, USA.

14. Centers for Disease Control and Prevention, National and state healthcare associated infections progress report, 2014, Atlanta, GA.

15. European Centers for Disease Control and Prevention, Point prevalence survey of healthcare-associated infections and antimicrobial use in European acute care hospitals, 2013, Stockholm, Sweden.

16. Davey, P., Brown, E., Charani, E., et al., Interventions to improve antibiotic prescribing practices for hospital inpatients. Cochrane Database of Systematic Reviews, 2013, (4).

17. Wentzel, J., Van Velsen, L., Van Limburg, M., et al., Participatory eHealth development to support nurses in antimicrobial stewardship. BMC medical informatics and decision making, 2014, 14(45).

18. National Quality Forum, National Quality Partners Playbook: Antibiotic stewardship in acute care, N.Q. forum, Editor 2016, National Quality forum: Washington.

19. Wentzel, J., De Jong, N., Karreman, J., et al., Implementation of MRSA Infection Prevention and Control Measures-What Works in Practice? In: Infection Control Updates. InTech. ISBN 9789535100553. 
20. Abbo, L., Smith, L., Pereyra, M., et al., Nurse practitioners' attitudes, perceptions, and knowledge about antimicrobial stewardship. The Journal for Nurse Practitioners, 2012, 8(5), p. 370-376.

21. Stach, L.M., Hedican, E.B., Herigon, J.C., et al., Clinicians' attitudes towards an antimicrobial stewardship program at a children's hospital. Journal of the Pediatric Infectious Diseases Society, 2012, p. pis045.

22. When the drugs don't work, 2016, The Economist, Retrieved from: http://www.economist.com/news/leaders/21699116-how-combat-dangerous-riseantibiotic-resistance-when-drugs-donu2019t-work.

23. Fishman, N., Antimicrobial stewardship. American Journal of Infection Control, 2006. 34(5), p. S55-S63.

24. Eccles, M., Grimshaw, J., Walker, A., et al., Changing the behavior of healthcare professionals: the use of theory in promoting the uptake of research findings. Journal of Clinical Epidemiology, 2005, 58(2), p. 107-112.

25. Hulscher, M.E., Grol, R.P., and Van Der Meer J.W., Antibiotic prescribing in hospitals: a social and behavioural scientific approach. The Lancet Infectious Diseases, 2010, 10(3), p. 167-175.

26. Janes, C.R., Corbett, K.K., Jones, J.H., et al., Emerging infectious diseases: the role of social sciences. The Lancet, 2012, 380(9857), p. 1884-1886.

27. Van Woezik, A.F., Braakman-Jansen, L.M.A., Kulyk, O., et al., Tackling wicked problems in infection prevention and control: a guideline for co-creation with stakeholders. Antimicrobial Resistance \& Infection Control, 2016, 5(20).

28. Harrison, M.I., Koppel, R., and Bar-Lev, S., Unintended consequences of information technologies in health care-an interactive sociotechnical analysis. Journal of the American Medical Informatics Association, 2007, 14(5), p. 542-549.

29. Eysenbach, G., What is e-health? Journal of medical Internet Research, 2001, 3(2), p. e20.

30. Köhle, N., Drossaert, C.H.C., Schreurs, K.M.G., et al., A web-based self-help intervention for partners of cancer patients based on Acceptance and Commitment Therapy: a protocol of a randomized controlled trial. BMC Public Health, 2015, 15(1), p. 1.

31. Bolier, J., Positive psychology online: using the internet to promote flourishing on a large scale, 2015, Universiteit Twente.

32. Cesa, G.L., Manzoni, G.M., Bacchetta, M., et al., Virtual Reality for Enhancing the Cognitive Behavioral Treatment of Obesity With Binge Eating Disorder: Randomized Controlled Study With One-Year Follow-up. Journal of Medical Internet Research, 2013, 15(6), p. e113.

33. Gorini, A., Gaggioli, A., and Riva, G., A second life for eHealth: prospects for the use of 3-D virtual worlds in clinical psychology. Journal of Medical Internet Research, 2008, 10(3), p. e21.

34. Nijland, N., Grounding eHealth: towards a holistic framework for sustainable eHealth technologies, 2011, University of Twente: Enschede.

35. Van Gemert-Pijnen, J.E.W.C., Niiland, N., Van Limburg, M., et al., A Holistic Framework to Improve the Uptake and Impact of eHealth Technologies. Journal of Medical Internet Research, 2011, 13(4), p. e111.

36. Shortliffe, T. and Davis, R., Some considerations for the implementation of knowledgebased expert systems. SIGART Bull., 1975, (55), p. 9-12.

37. Kelders, S.M., Understanding adherence to web-based interventions 2012: Universiteit Twente. 
38. Kelders, S.M., Kok, R.N., Ossebaard, H.C., et al., Persuasive system design does matter: a systematic review of adherence to web-based interventions. Journal of Medical Internet Research, 2012, 14(6), p. e152.

39. Bourgeois, F.C., Linder, J., Johnson, S.A., et al., Impact of a computerized template on antibiotic prescribing for acute respiratory infections in children and adolescents. Clinical Pediatrics, 2010, 49(10), p. 976-83.

40. Hum, R.S., Cato, K., Sheehan, B., et al., Developing clinical decision support within a commercial electronic health record system to improve antimicrobial prescribing in the neonatal ICU. Applied Clinical Informatics, 2014, 5(2), p. 368-387.

41. Niiland, N., Van Gemert-Piinen, J.E.W.C., Kelders, S.M., et al., Factors Influencing the Use of a Web-Based Application for Supporting the Self-Care of Patients with Type 2 Diabetes: A Longitudinal Study. Journal of Medical Internet Research, 2011, 13(3), p. e71.

42. Van De Belt, T.H., Engelen, L.J., Berben, S.A.A., et al., Definition of Health 2.0 and Medicine 2.0: A Systematic Review. Journal of Medical Internet Research, 2010, 12(2), p. e18.

43. Kukafka, R., Johnson, S.B., Linfante, A., et al., Grounding a new information technology implementation framework in behavioral science: a systematic analysis of the literature on IT use. Journal of Biomedical Informatics, 2003, 36(3), p. 218-227.

44. Bødker, K., Kensing, F., and Simonsen, J., Participatory IT design: designing for business and workplace realities, 2009: MIT press.

45. Van Der Vaart, R., Drossaert, C.H.C., Taal, E., et al., Patient preferences for a hospitalbased rheumatology Interactive Health Communication Application and factors associated with these preferences. Rheumatology, 2011, 50(9), p. 1618-1626.

46. Wentzel, J., Van Drie-Pierik, R., Nijdam, L., et al., Antibiotic information application offers nurses quick support. American Journal of Infection Control. 44(6), p. 677-684.

47. Wentzel, J., Limburg, A.H.M., Karreman, J., et al. Co-creation with stakeholders: A web 2.0 antibiotic stewardship program. In: Proceedings of The Fourth International Conference on eHealth, Telemedicine, and Social Medicine: January 30, 2012 to February 4, 2012; Valencia, Spain.

48. McColl, A., Smith, H., White, P., et al., General practitioners' perceptions of the route to evidence based medicine: a questionnaire survey. BMJ, 1998, 316(7128), p. 361-365.

49. Fogg, B.J., Persuasive technology: using computers to change what we think and do, Ubiquity, 2002, p. 5.

50. Oinas-Kukkonen, H., Behavior Change Support Systems: A Research Model and Agenda, In: $5^{\text {th }}$ International Conference on Persuasive Technology, 2010, Springer Berlin Heidelberg, p. 4-14.

51. Lehto, T., Oinas-Kukkonen, H., and Drozd, F., Factors Affecting Perceived Persuasiveness of a Behavior Change Support System. In: '33th International Conference on Information Systems, 2012, Association for Information Systems, Orlando, Florida.

52. De Jong, N., Wentzel, J., Kelders, S.M., et al., Evaluation of Perceived Persuasiveness Constructs by Combining User Tests and Expert Assessments. In: Second International Workshop on Behavior Change Support Systems, In conjunction with the 9th International Conference on Persuasive Technology, 2014, Padova, Italy. 



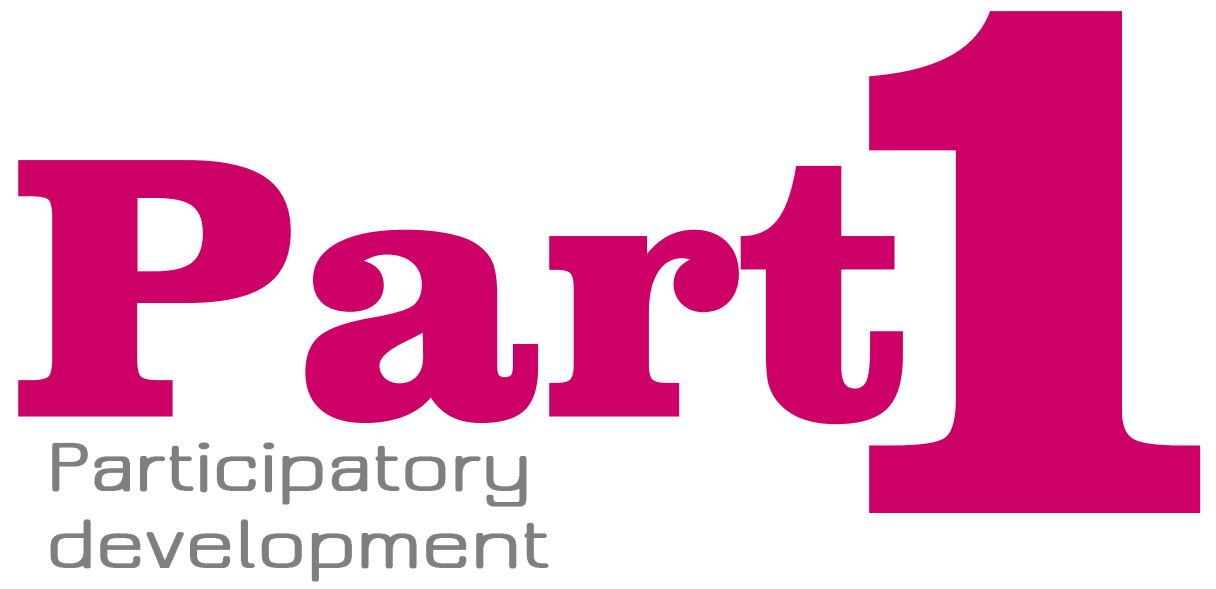




\title{
Case
}

\section{Clinical Decision Support Systems for antimicrobial prescribing}

\begin{abstract}
Although the concept of computerized Clinical Decision Support Systems to support the prudent prescribing of antimicrobials is a very broad umbrella term, and no widely accepted definition exists, it is most certainly not a new one. On the contrary, already in the early "70s Shortliffe et al. [1] in their study evaluated an "artificial intelligence program", which aimed at advising physicians regarding antimicrobial therapy. Remarkably, just a couple of years later the same authors report on "some problems of 'real-world' implementation for this kind of system" [2]. To date, successful implementation and uptake of these systems is still one of the main challenges for their success. The fact that these exact problems had already been reported on in the '70s, is illustrative of the complexity of the matter.
\end{abstract}

But what are Clinical Decision Support Systems (CDSSs) for antimicrobial prescribing? In short, it concerns an umbrella term for a system that uses logic (or: decision rules) to analyze patient data input to help clinicians make clinical decisions [3, 4]. CDSSs exist in many ways, shapes and forms. Although this thesis mainly focuses on computerized CDSSs, they are also available in paper-based form $[5,6]$. However, research has shown that these systems have been less effective in clinical practice [6]. Another factor that varies from system to system, is the amount of data input that is required or used. It may be elaborate or limited to one or two variables [7].

Although we have established that computerized CDSSs are not a new phenomenon, it seems that over the last decades more and more research has focused on them [3]. This is very likely related to the worldwide rise of antimicrobial resistance. The Centers for Disease Control and Management (CDC) in their recent report on "Core elements of hospital antibiotic stewardship programs" also underscored the importance of involving information technology staff in antimicrobial stewardship programs, to aid in the implementation of CDSSs for antimicrobial use [8]. 


\section{References}

1. Shortliffe, E.H. , Axline, S.G., Buchanan, B.G., et al., An Artificial Intelligence program to advise physicians regarding antimicrobial therapy. Computers and Biomedical Research, $1973,6(6)$, p. 544-560.

2. Shortliffe, T. and R. Davis, Some considerations for the implementation of knowledgebased expert systems. SIGART Bulletin, 1975, (55), p. 9-12.

3. Beerlage-de Jong, N., Wentzel, J., Dik, J.-W., et al., Opening the black box of Clinical Decision Support Systems for antimicrobial stewardship - A scoping review. International Journal of Medical Informatics, Submitted.

4. Kawamoto, K., Houlihan, C.A., Balas, E.A., et al., Improving clinical practice using clinical decision support systems: a systematic review of trials to identify features critical to success. BMJ, 2005, 330(7494), p. 765.

5. Samore, M.H., Bateman, K., Alder, S.C., et al., Clinical decision support and appropriateness of antimicrobial prescribing: A randomized trial. Jama, 2005, 294(18), p. 2305-2314.

6. Leung, G.M., Johnston, J.M., Tin, K.Y.K., et al., Randomised controlled trial of clinical decision support tools to improve learning of evidence based medicine in medical students. BMJ, 2003, 327(7423), p. 1090.

7. McGinn, T.G., McCullagh, L., Kannry, J., et al., Efficacy of an evidence-based clinical decision support in primary care practices: a randomized clinical trial. JAMA Internal Medicine, 2013, 173(17), p. 1584-1591.

8. Pollack, L.A. and A. Srinivasan, Core elements of hospital antibiotic stewardship programs from the Centers for Disease Control and Prevention. Clinical Infectious Diseases, 2014, 59(suppl 3), p. S97-S100. 



\section{Opening the black box of Clinical Decision Support Systems for antimicrobial stewardship - A scoping review}

N. Beerlage-de Jong, J. Wentzel, J.-W. Dik, B. Sinha,

R. Sanderman, L. Van Gemert-Pijnen, International Journal of Medical Informatics (submitted) 


\section{Abstract}

Clinical Decision Support Systems (CDSSs) are frequently used to support physicians in their daily practice, for example to prescribe antimicrobials prudently in an Antimicrobial Stewardship Program (ASP). CDSSs are usually developed expert- or policy-driven, and therefore might not optimally fit the clinical context in which they are used. Most studies on the use of these CDSSs mainly focus on the decision making using clinical values. Whether the system is supportive in doing so, is often neglected. The aim of this Scoping Review (a literature review method, suitable for broad fields of study and a focus on contextual information) is to gain insight into the values and requirements for developing usable and user-friendly CDSSs. Ultimately, the goal is to support decision making in ASP.

Scopus, Cochrane Library and PubMed were searched to identify relevant articles that (I) provide a description of the CDSS, (II) are aimed at appropriate antimicrobial prescribing, (III) concern a CDSS that is aimed at physicians, and (IV) report on behavioral, clinical, or financial outcome measures. Based on our scoped search query, a total of 22 articles were included for this study. Data were systematically extracted, collated, summarized and reported using a standardized extraction form and spreadsheet.

A broad diversity in CDSSs for the support of prudently prescribing antibiotics was found. The studied CDSSs provided support to (I) select microbiological tests, (II) select a (presumptive) diagnosis, (III) select an appropriate antibiotic, and / or (IV) offered 'other' support (e.g., patient information). Most of the studies did not or only scarcely report on how the CDSSs were developed and how the look-and-feel of the system was determined. More attention was paid to the implementation. Most of the articles applied one or more activities (e.g., demonstrations of the system, academic detailing) to support implementation of the CDSS. The articles evaluated the CDSSs via different outcome variables, but none of the included studies evaluated the system itself or the way it offers support.

Prior research has demonstrated that in order to achieve clinical success, it is paramount that CDSSs are easy to use. However, few of the included studies pay attention to the usability and user-friendliness of the CDSSs. We propose expanding an existing framework by Wright \& Sittig, for evaluation of CDS architectures. This expansion consists of crucial elements from the user-perspective, covering the usability and user-friendliness of CDSSs. 


\section{Background}

Antimicrobial Resistance (AMR) is a fast growing, major threat to patient safety in modern day health care [1]. A significant driver of AMR is the suboptimal use of antimicrobials. It is estimated that up to $50 \%$ of antimicrobial use is inappropriate, e.g., of a suboptimal kind, duration, dose or dosing regimen [2-5]. Thus, the prudent prescribing and use of antimicrobials is of paramount importance for dealing with the rapid development of AMR [6]. To support physicians in doing so, Antimicrobial Stewardship Programs (ASPs) are implemented in healthcare institutions. Aim of ASPs is "ensuring effective treatment of patients with infection and minimizing collateral damage from antimicrobial use [6]. Collateral damage relates to e.g., toxicity or side effects and enhancing AMR.

Implementing an ASP, requires different healthcare professionals (e.g., prescribing physicians, microbiologists, pharmacists) and different institutions (e.g., hospital, laboratory, pharmacy) to closely, constructively and rapidly cooperate. In our view, applying (eHealth) technology is very suitable to support such interdisciplinary and cross institutional cooperation [7,8]. For example, an eHealth technology was developed and is used to support nurses in prescribing antimicrobials by offering them easy access to prescribing information [9]. eHealth technology allows for and stimulates (real-time) data and knowledge exchange, and it offers a means for communicating understandable and easily accessible information and instructions $[8,10,11]$.

One such technology, which has frequently been studied and applied in practice, is a Clinical Decision Support System (CDSS). Many different definitions of CDSSs exist [12]. For example, Kawamoto et al. [13] define CDSSs as "any electronic or non-electronic system designed to aid in clinical decision making, in which characteristics of individual patients are used to generate patient-specific assessments or recommendations that are then presented to clinicians for consideration". In ASPs, these systems can offer support in prudently prescribing antimicrobials [14].

Concerning CDSSs, Wright and Sittig [15] mention that "the people who develop a clinical system (often computer programmers) and the people who develop the decision support content (usually clinicians and information specialists) are frequently different". This implies and underscores our belief, that the term 'Clinical Decision Support Systems' actually consists of two parts, i.e., a decision that is made based on support content, and a system that is used to come to that decision. Most research focuses on the effect of the 'Decision content' part of CDSSs, i.e., they focus on the clinical effectiveness that is realized by a CDSS in clinical practice. At the same time, the 'Support System' part of CDSSs is in most cases overlooked in literature.

Yet, prior research has shown that for CDSSs to be successful, it is of paramount importance that they are easy to use [13], by e.g., being integrated in workflow, enabling timely decision support. Thus, to what extend a CDSS is usable and user-friendly are key factors for its success. Even more so, considering the fact that adherence to these kinds of systems is notoriously challenging. Unfortunately, only a few existing papers cover the use and user-centeredness of CDSSs for ASP $[14,16]$. Therefore, to explore this aspect of CDSSs for ASP, the current scoping review is done as part of a Contextual Inquiry [8, 17]. Meaning that its aim is to gain insight into the values and requirements for developing usable and user friendly eHealth technology, to support decision making in ASP. Ultimately, we strive to enable the emergence of CDSSs that fit their users and use context. 
With this paper, we do not seek to evaluate the success of existing CDSSs for ASP or to evaluate the study quality of found articles. Rather, we want to gain insight in the many different varieties of CDSSs for ASP that exist, and in what was done to make them as supportive as possible. That is also why we chose to perform a scoping literature review, rather than a systematic literature review or meta-analysis [18]. This approach allows researchers to focus on (contextual) information about the CDSS itself, and about its development, design and implementation. In this manner, insight is gained in how a CDSS can support healthcare providers in the decision making within an ASP, and how these systems can be developed or improved to enlarge its supportiveness. Thus, with this paper, we want to provide a framework for the development of usable and user friendly CDSSs for ASP. Therefore, the main research question of this review is:

How user-centered and supportive are Clinical Decision Support Systems for Antimicrobial Stewardship in practice?

\section{Methods}

\subsection{Study design}

Aim of this paper is to formulate values and requirements for the development or improvement of CDSSs for ASP, so that they are usable and user-friendly. This is done as part of a Contextual Inquiry [17]. As this already implies, we are currently 'scoping the field'. To do so, this scoping literature study consisted of five stages, based on the methodological framework by Arksey \& O'Malley: (I) identifying the research question; (II) identifying relevant studies; (III) study selection; (IV) charting the data; and (V) collating, summarizing and reporting the results [18].

\subsubsection{Identifying the research question[s]}

The main research question is answered by focusing on six aspects of CDSSs, to describe how they have come to be and how they intent to offer support to its users: (I) what are Clinical Decision Support Systems for Antimicrobial Stewardship Programs (ASPs) - how are they described and defined, (II) how are they developed, (III) how are they designed, (IV) what kind of support do they offer, (V) how are they implemented, and (VI) how are their effects measured.

\subsubsection{Identifying relevant studies}

Scopus (covering the field of health sciences), PubMed (covering the field of (bio)medical and life sciences) and Cochrane Library (for clinical trials and health technology assessments) were searched for relevant literature about (the use of) existing CDSSs for antimicrobial prescribing. The search query was deliberately composed using very broad terms, in order to generate breadth of coverage [18]. The search query consisted of a combination of different keywords, given in Table 1. 
Table 1: Search query of the scoping review

\begin{tabular}{ll}
\multicolumn{1}{c}{ Field } & \multicolumn{1}{c}{ Keywords } \\
\hline Title-abs-key & "anti*bacterial*" OR "antibiotic*" OR "antimicrobial*" \\
\hline AND & \\
\hline Title-abs-key & "clinical decision support" OR "clinical decision aid" OR "CDS" OR "CDSS" \\
AND & \\
\hline Pubyear & $>1999$ \\
\hline
\end{tabular}

\subsubsection{Study selection}

Article inclusion consisted of three stages: (I) title, (II) abstract, and (III) full text screening (see Figure 1). Articles that were included had the following characteristics: scientific articles; written in English; reporting on primary data; providing a clear description of the CDSS, aimed at prudent antimicrobial prescribing (in both in- and outpatient settings); the CDSS has been implemented; the CDSS is aimed at physicians; behavioral, clinical, or financial outcome measures are reported. If inclusion of an article was doubtful, it was discussed with fellow researchers.

\subsubsection{Charting the data}

The next stage in the scoping review involved 'charting' the data. For this scoping study, an approach that is 'akin to a narrative review' was used, following Arksey and O'Malley [18]. By using this approach, more contextual information about the underpinning of the intervention and its development and design process is extracted in detail. Less emphasis is placed on the impact and effects of the CDSSs. Data were charted about the study design, the development of the system, its look-and-feel and characteristics (e.g., aim of the support), and activities which were undertaken for its implementation.

\subsubsection{Collating, summarizing and reporting the results}

In the stage of collating, summarizing and reporting the results, emphasis is placed on gaining more insight into the field of study, and in the different kinds of CDSSs that are found within the research scope (as can be the case in qualitative studies). The data were collected in a template (in the form of an Excel worksheet). Literature was summarized thematically, based on the various topics of interest. 
Search query in PubMed,

Scopus \& Cochrane Library $(n=620)$

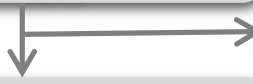

Duplicates removed $(n=169)$

Publications entered in title screening

Publications excluded in title screening ( $n=323$ )

Publications entered in abstract screening $(n=128)$

Publications entered in full-text screening $(n=49)$

Publications excluded in abstract screening $(n=79)$

- No CDSS implemented $(n=30)$

- No ASP ( $n=22)$

- Systematic Review ( $n=13$ )

- No Outcome measures $(n=8)$

- No scientific article $(n=6)$

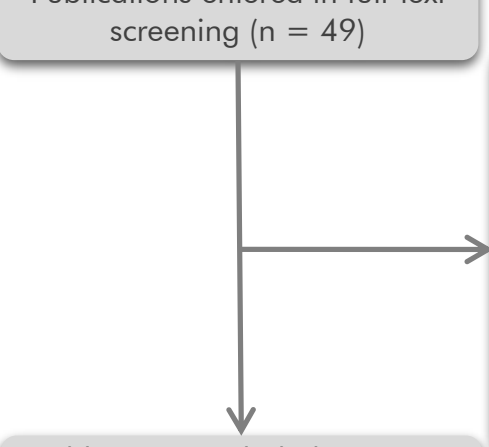

Publications included in review

Publications excluded in full-text screening $(n=27)$

- No ASP $(n=5)$

- No CDSS implemented $(n=6)$

- Not aimed at physicians $(n=4)$

- No description of CDSS $(n=1)$

- No scientific article $(n=2)$

- No outcome measures $(n=4)$

- Pilot test for other article $(n=1)$

- No primary data $(n=2)$

- No full-text available $(n=2)$

$$
(n=22)
$$

Figure 1: Graphical overview of the study selection process

\section{Findings}

Out of 620 identified studies (including 169 duplicates), 22 studies were included in the final scoping review, which was performed on February $8^{\text {th }} 2016$ [19-36]. An overview of the characteristics of the included studies is given in Table 2 . Most (72\%) of the studies are performed in the USA $[19,21,23-30,32-34,36-38]$ and most $(86 \%)$ were published in 2008 or later [19-28, 31, 33-40].

\subsection{How are Clinical Decision Support Systems defined and described?}

The 22 studies that were included in this scoping review rarely explicitly mentioned how they define their CDSS. Only two of the studies [27, 29] explicitly provide a definition of a CDSS. Within one of those studies, it is stated that the authors focus on "Computerized Clinical Decision-Support Systems (CDSSs), which integrate clinical, patient, and reference information to provide assistance with decision-making in patient care" [29]. Within the 
other study, it is mentioned that "most forms of CDS involve input of 1 or 2 simple data points. (...) More complex forms of decision support that are customized and pull data from various data fields" [27].

Although all included studies are about a CDSS, these CDSSs differ quite a lot (concerning development, design and content). Additionally, the descriptions of the CDSSs that were available were often quite brief and (medical) content-focused. They mainly focus on the decision support content (as specified by Wright and Sittig [15]: the application of clinical rules, protocols or guidelines to predict and advice about the likelihood of an infection or appropriate therapy. Thus, descriptions of CDSSs tend to neglect the support that is given (and how) and the role the system plays in doing so.

Working with and reporting on a clear definition of a CDSS, would help researchers in developing and describing their system. It allows other researchers to determine which definition would fit their own needs and expectations, so they can learn from the description and results of existing CDSSs. 


\begin{tabular}{|c|c|c|c|c|c|}
\hline Author(s) & $\begin{array}{l}\text { Year, } \\
\text { Country }\end{array}$ & Study design & Setting & N Pts/Enc & N Providers \\
\hline Agwu et al. & $\begin{array}{l}2008 \\
\text { USA }\end{array}$ & Before-and-after & $\begin{array}{l}\text { 175-bed tertiary care children's } \\
\text { hospital }\end{array}$ & 4104 requests & 121 (survey) \\
\hline $\begin{array}{l}\text { Bourgeois } \\
\text { et al. }\end{array}$ & $\begin{array}{l}2010 \\
\text { USA }\end{array}$ & Case-Control & 12 ambulatory pediatric clinics & $\begin{array}{l}9409 \text { pts in intervention-, } \\
\text { and } 2907 \text { pts in control- } \\
\text { group }\end{array}$ & N/A \\
\hline $\begin{array}{l}\text { Buising et } \\
\text { al. }\end{array}$ & $\begin{array}{l}2008 \\
\text { AUS }\end{array}$ & $\begin{array}{l}\text { Two-stage pre- and } \\
\text { post-intervention } \\
\text { cohort }\end{array}$ & $\begin{array}{l}\text { ED of a } 350 \text {-beds urban adult } \\
\text { tertiary teaching hospital }\end{array}$ & $\begin{array}{l}\text { Baseline: } 392 \text { pts; } \\
\text { Academic detailing: } 215 \\
\text { pts; Intervention group: } \\
133 \text { pts. }\end{array}$ & N/A \\
\hline $\begin{array}{l}\text { Carman et } \\
\text { al. }\end{array}$ & $\begin{array}{l}2011 \\
\text { USA }\end{array}$ & $\begin{array}{l}\text { Quasi-experimental, } \\
\text { pretest-posttest }\end{array}$ & $\begin{array}{l}1 \text { level-one trauma center with } \\
\text { adult and pediatric EDs, } 1 \\
\text { community hospital ED and } 2 \\
\text { freestanding EDs }\end{array}$ & 873 encounters & N/A \\
\hline $\begin{array}{l}\text { Charani et } \\
\text { al. }\end{array}$ & 2013, UK & In-depth case-study & $\begin{array}{l}5 \text { teaching hospitals at three } \\
\text { sites }\end{array}$ & $\mathrm{N} / \mathrm{A}$ & $\begin{array}{l}\text { Pre-intervention: } 71 \\
\text { dctrs, } 16 \text { pharm; post- } \\
\text { intervention: } 81 \text { dctrs }\end{array}$ \\
\hline Chow et al. & $\begin{array}{l}2015 \\
\text { Singa- } \\
\text { pore }\end{array}$ & $\begin{array}{l}\text { Prospective } \\
\text { observational cohort }\end{array}$ & $\begin{array}{l}\text { 1500-bed academic tertiairy } \\
\text { care hospital }\end{array}$ & 1886 pts & $\mathrm{N} / \mathrm{A}$ \\
\hline Dean et al. & $\begin{array}{l}2015 \\
\text { USA }\end{array}$ & $\begin{array}{l}\text { Prospective quasi- } \\
\text { experimental controlled } \\
\text { trial }\end{array}$ & 7 EDs in 7 different hospitals & $4758 \mathrm{pts}$ & $\mathrm{N} / \mathrm{A}$ \\
\hline $\begin{array}{l}\text { Demonchy } \\
\text { et al. }\end{array}$ & 2014, FR & $\begin{array}{l}\text { Prospective before- } \\
\text { and-after controlled } \\
\text { interventional study }\end{array}$ & 3 EDs in 3 different hospitals & $912 \mathrm{pts}$ & $\mathrm{N} / \mathrm{A}$ \\
\hline Filice et al. & $\begin{array}{l}2013 \\
\text { USA }\end{array}$ & Retrospective cohort & $\begin{array}{l}\text { 279-bed university-affiliated } \\
\text { Dept. of Veterans Affairs } \\
\text { hospital }\end{array}$ & $\begin{array}{l}500 \text { pts who received an } \\
A B \text { therapy }\end{array}$ & $\mathrm{N} / \mathrm{A}$ \\
\hline $\begin{array}{l}\text { Hingorani } \\
\text { et al. }\end{array}$ & $\begin{array}{l}2015 \\
\text { USA }\end{array}$ & Before-and-after & $\begin{array}{l}\text { Internal medicine department } \\
\text { in Reading }\end{array}$ & 240 enc & N/A \\
\hline
\end{tabular}




\begin{tabular}{|c|c|c|c|c|c|}
\hline $\begin{array}{l}\text { Hulgan et } \\
\text { al. }\end{array}$ & $\begin{array}{l}2004 \\
\text { USA }\end{array}$ & Interrupted time-series & $\begin{array}{l}\text { University hospital in the south- } \\
\text { eastern United States }\end{array}$ & 15194 quinolone orders & $\mathrm{N} / \mathrm{A}$ \\
\hline Hum et al. & $\begin{array}{l}2014 \\
\text { USA }\end{array}$ & $\begin{array}{l}\text { Post-intervention survey } \\
\text { and log-data }\end{array}$ & $\begin{array}{l}2 \text { NICUs with a total of } 107 \\
\text { beds }\end{array}$ & 2009 pts & 83 dctrs \\
\hline Linder et al. & $\begin{array}{l}2009 \\
\text { USA }\end{array}$ & Clustered RCT & 27 primary care clinics & 21961 visits & 443 dctrs \\
\hline Litvin et al. & $\begin{array}{l}2012 \\
\text { USA }\end{array}$ & Before-and-after & $\begin{array}{l}9 \text { primary care practices, } \\
\text { representing } 27 \text { physicians, } 6 \\
\text { nurses and } 6 \text { physicians' } \\
\text { assistants. }\end{array}$ & 38592 ARI visits & N/A \\
\hline $\begin{array}{l}\text { Mainous et } \\
\text { al. }\end{array}$ & $\begin{array}{l}2012 \\
\text { USA }\end{array}$ & Quasi-experimental & 70 primary care practices & $\mathrm{N} / \mathrm{A}$ & $\begin{array}{l}9 \text { intervention- and } 61 \\
\text { control-practices }\end{array}$ \\
\hline $\begin{array}{l}\text { McGinn et } \\
\text { al. }\end{array}$ & $\begin{array}{l}2013 \\
\text { USA }\end{array}$ & Case-Control & $\begin{array}{l}2 \text { large urban ambulatory } \\
\text { primary care practices }\end{array}$ & 984 pts & $\mathrm{N} / \mathrm{A}$ \\
\hline $\begin{array}{l}\text { Nachtigall } \\
\text { et al. }\end{array}$ & 2014, GE & $\begin{array}{l}\text { Prospective before- } \\
\text { and-after cohort }\end{array}$ & $\begin{array}{l}5 \mathrm{ICUs}(61 \mathrm{beds}) \text { of } 3200-\text { bed } \\
\text { tertiary care medical centre }\end{array}$ & 1316 pts & N/A \\
\hline $\begin{array}{l}\text { Rattinger et } \\
\text { al. }\end{array}$ & $\begin{array}{l}2012 \\
\text { USA }\end{array}$ & Before-and-after & $\begin{array}{l}\text { Veterans affairs Health Care } \\
\text { System }\end{array}$ & $\begin{array}{l}3831 \text { unique pts with } \\
\text { initial ARI visit }\end{array}$ & N/A \\
\hline Rubin et al. & $\begin{array}{l}2006 \\
\text { USA }\end{array}$ & Observational & $\begin{array}{l}\text { Primary care providers in } 6 \\
\text { rural communities }\end{array}$ & N/A & $\begin{array}{l}99 \text { primary care } \\
\text { providers }\end{array}$ \\
\hline $\begin{array}{l}\text { Samore et } \\
\text { al. }\end{array}$ & $\begin{array}{l}2005 \\
\text { USA }\end{array}$ & $\begin{array}{l}\text { Cluster randomized } \\
\text { trial }\end{array}$ & $\begin{array}{l}\text { Primary care providers in } 12 \\
\text { study, and } 6 \text { non-study } \\
\text { communities }\end{array}$ & $\mathrm{N} / \mathrm{A}$ & 176 primary care detrs \\
\hline $\begin{array}{l}\text { Tafelski et } \\
\text { al. }\end{array}$ & $\begin{array}{l}2010 \\
\text { DU }\end{array}$ & $\begin{array}{l}\text { Pre- / post-intervention } \\
\text { cohort }\end{array}$ & $\begin{array}{l}3 \text { closed ICUs of a } 3200-\text { bed } \\
\text { community acute-care } \\
\text { university hospital, providing } \\
\text { tertiary care }\end{array}$ & 977 pts & $\mathrm{N} / \mathrm{A}$ \\
\hline $\begin{array}{l}\text { Vincent et } \\
\text { al. }\end{array}$ & $\begin{array}{l}2009 \\
\text { USA }\end{array}$ & Case-Control & $\begin{array}{l}\text { 473-bed, tertiary academic } \\
\text { medical center }\end{array}$ & $\begin{array}{l}48 \text { pts in study-, } 49 \text { pts in } \\
\text { control group }\end{array}$ & N/A \\
\hline
\end{tabular}

ED: Emergency Department, ICU: Intensive Care Unit, Pts: patients, Enc: encounters, Dctrs: doctors, Pharm: pharmacists, ARI: Acute Respiratory Infections. 


\subsection{How are CDSSs for ASPs developed?}

The development process of (eHealth) technology is of great importance for its success [17]. Therefore, we focused on what the studies report about the development process of the CDSS (see also Table 3). Information about the development is scarce, half of the articles do not report on how the CDSS was developed [19, 20, 23, 28-30, 34-36, 41]). There are articles which do mention some aspects of the development process (e.g., who were involved, what the system is based on), but they do so very briefly [21, 22, 25-27, 31-33, 37-40], e.g., mentioning that "the Department of Biomedical Informatics (DBMI) at Vanderbilt University Medical Center (VUMC) has developed" the system [32]. Most CDSSs are developed based on a policy (a guideline or protocol about prescribing antibiotics) that must be communicated [21, 27], based on a collaboration between experts [32,33], or based on a combination of the above [25, 31].

Potential end-users are included in the development of the CDSS by a minority of the included studies. However, the more recent studies (2013-2015) tend to more often involve them (practice representatives / -liaisons) [22, 26, 37-40]. This is most often combined with a CDSS that is developed based on an existing policy [22, 37-40]. This suggests that in the past and even (to some extent) to date, CDSSs are usually developed expert- or policy-driven, and therefore might not optimally fit the clinical context which they are used in.

\subsection{How are CDSSs for ASPs designed?}

The design (e.g., layout, look and feel) of eHealth technologies should be considered to ensure its success [17]. Thus, we investigated what is reported about the design (process) of the CDSSs for ASPs (see also Table 3).

Information thereon is scarce. In fact, nine articles do not report anything about the design of the CDSS [20, 23, 28-30, 33-36]. Some articles [21, 22, 26, 27, 32, 37, 38, 40] do provide screenshots, but these are often unclear (due to suboptimal quality or limited amount of screenshots), so they are still limited in the information they provide about the design of the CDSS [21, 22, 26, 27, 32]. Two articles provide a link to (a standalone demo version of) the CDSS, but one of these does not work (website does not exist) [19] and the other one is in French [39], creating a barrier to view and use them.

This apparent lack of attention for the design of the CDSSs may be a missed opportunity. Prior research on the persuasiveness of technology has shown that, as Oinas-Kukkonen and Harjumaa put it, "technology is never neutral" [42]. This implies that the technology (and its design) can play an important role in its success in supporting ASPs.

One study [31] does explicitly mention the design choices that were made, namely that "the pathways (of their CDSS, eds.) were translated into a web-based form, with similarities to common browser systems, thereby the system was familiar and convenient to users" [31]. However, the study did not report on user-testing this 'familiarity' and 'convenience' among potential end-users. Another study [22] provides multiple clear (black \& white) screenshots of their CDSS and explicitly mentions the involvement of the organization's communication team and an application designer ("a person who plans the look or workings of something prior to it being made, by preparing drawings or plans" [43]) in the development of the CDSS [22]. 
Table 3: Description of the CDSSs, and their development, design

\begin{tabular}{|c|c|c|c|c|c|}
\hline \multicolumn{4}{|c|}{ Characteristics of the CDSS } & \multirow[t]{2}{*}{ Development info given } & \multirow[t]{2}{*}{ Design info given } \\
\hline Author(s) & Aim of the support & $\begin{array}{l}\text { Specificity of } \\
\text { support }\end{array}$ & $\begin{array}{l}\text { Decision CDSS } \\
\text { generated }\end{array}$ & & \\
\hline Agwu et al. & $\mathrm{AB}$ selection & General & No & Expert collaboration & No \\
\hline $\begin{array}{l}\text { Bourgeois et } \\
\text { al. }\end{array}$ & $\begin{array}{l}\text { Lab test-, AB-selection, } \\
\text { Pt information }\end{array}$ & ARI & Yes & No & $\begin{array}{l}\text { Web link (does not } \\
\text { work) }\end{array}$ \\
\hline Buising et al. & $\begin{array}{l}\text { Diagnosis-, AB-selection, Other (Info on } \\
\text { unusual pathogens, medical literature) }\end{array}$ & CAP, ED & Yes & No & No \\
\hline Carman et al. & $\begin{array}{l}\text { Lab test-, AB-selection, Other (Easy } \\
\text { access to ordering tests) }\end{array}$ & $\begin{array}{l}\text { CA-MRSA related } \\
\text { SSTIs in ED }\end{array}$ & Yes & Guideline & $\begin{array}{l}\text { Single / unclear } \\
\text { screenshot }\end{array}$ \\
\hline Charani et al. & Lab test-, Diagnosis-, AB-selection & General & Yes & $\begin{array}{l}\text { Expert and stakeholder } \\
\text { collaboration, guidelines }\end{array}$ & $\begin{array}{l}\text { Screenshots \& } \\
\text { designer involved }\end{array}$ \\
\hline Chow et al. & AB-selection & Specific AB & Yes & No & No \\
\hline Dean et al. & $\begin{array}{l}\text { Lab test-, Diagnosis-, AB-selection, } \\
\text { other (Site of care) }\end{array}$ & Pneumonia in EDs & Yes & $\begin{array}{l}\text { Expert-, stakeholder-, end- } \\
\text { user collaboration, } \\
\text { guidelines }\end{array}$ & Screenshots \\
\hline $\begin{array}{l}\text { Demonchy et } \\
\text { al. }\end{array}$ & Lab test-, Diagnosis-, AB-selection & UTIs in EDs & Yes & $\begin{array}{l}\text { Expert collaboration, } \\
\text { guidelines }\end{array}$ & Link \\
\hline Filice et al. & $\mathrm{AB}$ selection & General & Yes & No & No \\
\hline $\begin{array}{l}\text { Hingorani et } \\
\text { al. }\end{array}$ & Diagnosis selection & ARI & Yes & No & No \\
\hline Hulgan et al. & $A B$ selection & Specific AB & Unknown & Collaboration & $\begin{array}{l}\text { Single / unclear } \\
\text { screenshot }\end{array}$ \\
\hline Hum et al. & $\mathrm{AB}$ selection & $\mathrm{HAl}$ in $\mathrm{NICU}$ & Yes & $\begin{array}{l}\text { Expert-, stakeholder-, and } \\
\text { end-user collaboration, } \\
\text { guidelines }\end{array}$ & Screenshots \\
\hline Linder et al. & $\begin{array}{l}\text { Lab test-, Diagnosis-, AB-selection, } \\
\text { Other (Patient handouts, Info on } \\
\text { potential interactions or allergies) }\end{array}$ & ARI & Yes & No & $\begin{array}{l}\text { Described } \\
\text { elsewhere }\end{array}$ \\
\hline
\end{tabular}




\begin{tabular}{|c|c|c|c|c|c|}
\hline Litvin et al. & Lab test-, Diagnosis-, AB-selection & ARI & Yes & $\begin{array}{l}\text { Expert collaboration, } \\
\text { guidelines }\end{array}$ & $\begin{array}{l}\text { Described } \\
\text { elsewhere }\end{array}$ \\
\hline $\begin{array}{l}\text { Mainous et } \\
\text { al. }\end{array}$ & $\begin{array}{l}\text { Diagnosis-, AB-selection, Other } \\
\text { (Printable patient and provider } \\
\text { education) }\end{array}$ & General & Yes & Expert collaboration & $\begin{array}{l}\text { Single / unclear } \\
\text { screenshot }\end{array}$ \\
\hline McGinn et al. & Lab test-, Diagnosis-, AB-selection & $\begin{array}{l}\text { Pneumonia or } \\
\text { pharyngitis }\end{array}$ & Yes & Existing CPR & $\begin{array}{l}\text { Single / unclear } \\
\text { screenshot }\end{array}$ \\
\hline $\begin{array}{l}\text { Nachtigall et } \\
\text { al. }\end{array}$ & $\begin{array}{l}\text { Lab test-, Diagnosis-, AB-selection, } \\
\text { Other (Link to references, background } \\
\text { info, local resistance patterns) }\end{array}$ & $\begin{array}{l}\text { Relevant surgical } \\
\text { ICU infections }\end{array}$ & Yes & $\begin{array}{l}\text { Expert- and end-user } \\
\text { collaboration, guidelines }\end{array}$ & Screenshots \\
\hline $\begin{array}{l}\text { Rattinger et } \\
\text { al. }\end{array}$ & Diagnosis-, AB-selection & ARI & Yes & No & No \\
\hline Rubin et al. & Diagnosis-, $A B$-selection & General & Yes & No & No \\
\hline Samore et al. & Diagnosis-, AB-selection & General & Yes & No & No \\
\hline Tafelski et al. & Lab test-, Diagnosis-, AB-selection & Sepsis & Yes & $\begin{array}{l}\text { Expert collaboration, } \\
\text { guidelines }\end{array}$ & $\begin{array}{l}\text { Clear description } \\
\text { of design }\end{array}$ \\
\hline Vincent et al. & Lab test-, AB-selection & General & No & No & No \\
\hline
\end{tabular}

ARI: Acute Respiratory Infections, CAP: Community-Acquired Pneumonia, ED: Emergency Department, CA-MRSA: Community-Acquired Methicillin-resistant Staphylococcus aureus, SSTI: Skin and Soft Tissue Infection, AB: Antibiotics, CPR: Clinical Prediction Rule. 


\subsection{What kind of support do the CDSSs for ASP offer?}

\subsubsection{Aim of the support}

To optimally counteract the development of antimicrobial resistance, we have proposed an integrated stewardship model wherein antimicrobial stewardship, infection prevention stewardship, and diagnostic stewardship are combined (in an AID stewardship model) and are mutually reinforcing [44]. To the best of our knowledge, CDSSs which incorporate all three aspects of infection management are scarce. Thus, in our paper we did not include the infection prevention stewardship. We focused on CDSSs which offer support for making an appropriate diagnosis and/or for optimally prescribing antibiotics. Yet, the AID model does subscribe our view that offering support at multiple aspects of the care process is most beneficial for patient safety $[7,9,45]$.

In the current scoping literature review (mainly based on the content of the included studies), we made a distinction between support (1) to select adequate microbiological laboratory tests, (2) to select a correct diagnosis, (3) to select an appropriate antimicrobial, and (4) 'other' support (e.g., patient information, easy access to scientific literature) (Table 3). As mentioned before, it is preferable that a combination of multiple of these decisions and functionalities is combined within a CDSS, to provide optimal support during the entire care process.

Most of the studied Clinical Decision Support Systems indeed offered a combination of two or more of the possible support types [19-22, 24-31, 34, 37, 39, 40]. Some even offer the full scope of support to select laboratory tests, diagnosis and antimicrobials (sometimes completed with 'other' support) $[22,24,25,27,31,37,39,40]$. This means that these CDSSs offer support on multiple aspects of ASPs. Yet, some of the studied CDSSs offer a single type of support $[23,32,33,35,36,38]$, thereby supporting only part of the ASP process.

\subsubsection{Specificity of the support: generic and specific}

The focus of some of the studied CDSSs is generic [22, 23, 26, 29, 30, 33, 34]: offering support for prescribing all kinds of antimicrobials, for all kinds of infections (see also Table 3 ). The other CDSSs offer specific support [19-21, 24, 25, 28, 31, 32, 35-40]: aiming at a specific (site of) infection (e.g., acute respiratory infections) or antimicrobial (e.g., ciprofloxacin), sometimes in combination with a focus on a specific department (e.g., Emergency Department) (Table 3).

In the current review, we included all kinds of CDSSs for ASPs. Yet, since the scopes of these systems differ, their impact and effects may differ as well. This is for example especially true for CDSSs that are used in an Emergency Department setting [20, 21, 37, 39], since their patients are highly heterogeneous and may require swift and aggressive treatment.

\subsubsection{Decision making via the CDSSs}

Most of the studied CDSSs automatically generate a treatment advice, based on programmed algorithms [19-32, 35-40] (Table 3). The others use the CDSS essentially as a tool to facilitate communication between prescribing physicians and a supporting pharmacist, by efficiently documenting the rationales for antimicrobial requests [33] and the subsequent advice that is given by a pharmacist [34] (Table 3). 


\subsection{How are CDSSs for ASPs implemented?}

The implementation of eHealth technology is an important aspect of its success [17]. Most of the included studies [19-21, 24-27, 31, 33, 36, 37, 39, 40] do report on applying one or more implementation strategies (see also Table 4). They offer some kind of education or training to introduce the CDSS into clinical practice. Of these studies, most use multifaceted implementation strategies [19, 21, 24, 25, 27, 29-31, 33, 36, 37, 40]. Combining multi-faceted implementation strategies has previously been shown to indeed be most effective $[17,46]$, although this does not necessarily mean it is also most efficient. Three studies provide a single-faceted implementation strategy [20, 26, 39]. Seven studies [22, $23,28,32,34,35,38$ ] do not report on applying any implementation strategy at all (which might not necessarily mean they did not offer it). Overall, giving demonstrations of how and when to work with the CDSS system is by far the most often used training method [19-21, 24-27, 31, 33, 39, 40].

Additionally, to optimize implementation in the care process, most of the studied CDSSs for ASPs were computer-based [19-21, 23-28, 31-40]. The use of computer-based CDSSs is in line with the results of a previously performed RCT by Leung et al., suggesting that paper-based CDSSs are indeed less effective than CDSSs that are used on a handheld computer [47]. The CDSSs which used a handheld computer, were a native Smartphone app [22], or standalone Personal Digital Assistant (PDA)-based CDSS [29, 30].

Of the computer-based systems, fifteen were integrated in the hospital's Electronic Health Record [19-21, 23-28, 37-39] or Computerized Physician Order Entry system [32, 34, 35]. Both research [15] and practice have shown that such integration of different technologies is not always an easy task. However, none of the studies report on any difficulties or barriers that they encountered. Insight into the experiences of others, would again contribute to advancing this field of study. 
Table 4: Implementation activities for and integration in care process of CDSSs in ASP

\begin{tabular}{|c|c|c|c|c|c|c|c|c|c|c|c|c|}
\hline \multirow[t]{2}{*}{ Article } & \multicolumn{3}{|c|}{ Introduction } & \multicolumn{3}{|c|}{ Demonstration } & \multicolumn{2}{|c|}{ Reminder } & \multicolumn{2}{|c|}{ ASP } & \multirow{2}{*}{ 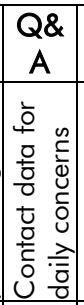 } & \multirow[t]{2}{*}{ Device } \\
\hline & $\begin{array}{l}\frac{0}{2} \\
\frac{1}{0} \\
\frac{1}{\overline{0}} \\
\overline{\overline{0}} \\
\frac{0}{0} \\
>\end{array}$ & 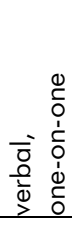 & $\begin{array}{l}0 \\
0 \\
0 \\
0 \\
0 \\
0 \\
0 \\
0 \\
3 \\
3\end{array}$ & 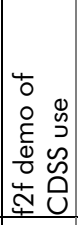 & 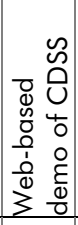 & 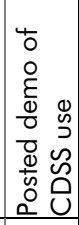 & 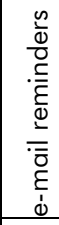 & 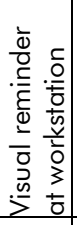 & 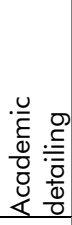 & 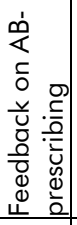 & & \\
\hline Agwu et al. & + & - & - & + & - & + & - & - & - & - & - & $\begin{array}{l}\text { Web-based; } \\
\text { broadband laptop }\end{array}$ \\
\hline $\begin{array}{l}\text { Bourgeois et } \\
\text { al. }\end{array}$ & - & - & - & + & - & - & + & - & - & - & - & $\begin{array}{l}\text { PC-based, } \\
\text { EHR-integrated }\end{array}$ \\
\hline Buising et al. & - & - & - & + & - & - & - & - & - & - & - & $\begin{array}{l}\text { PC-based, } \\
\text { EHR-integrated; web- } \\
\text { based }\end{array}$ \\
\hline Carman et al. & - & - & + & - & + & - & - & - & - & - & - & $\begin{array}{l}\text { PC-based, } \\
\text { EHR-integrated }\end{array}$ \\
\hline Charani et al. & - & - & - & - & - & - & - & - & - & - & - & $\begin{array}{l}\text { Native, smartphone } \\
\text { app }\end{array}$ \\
\hline Chow et al. & - & - & - & - & - & - & - & - & - & - & - & $\begin{array}{l}\text { PC-based, CPOE- } \\
\text { integrated }\end{array}$ \\
\hline Dean et al. & + & - & - & - & - & - & + & - & + & - & + & $\begin{array}{l}\text { PC-based, EMR- } \\
\text { integrated }\end{array}$ \\
\hline $\begin{array}{l}\text { Demonchy et } \\
\text { al. }\end{array}$ & - & - & - & + & - & - & - & - & - & - & - & $\begin{array}{l}\text { PC -based, EMR- } \\
\text { integrated }\end{array}$ \\
\hline Filice et al. & - & - & - & - & - & - & - & - & - & - & - & $\begin{array}{l}\text { PC -based, EHR- } \\
\text { integrated }\end{array}$ \\
\hline $\begin{array}{l}\text { Hingorani et } \\
\text { al. }\end{array}$ & - & - & - & - & - & - & + & + & + & + & - & PC -based \\
\hline Hulgan et al. & - & - & - & - & - & - & - & - & - & - & - & $\begin{array}{l}\text { PC -based, CPOE- } \\
\text { integrated }\end{array}$ \\
\hline Hum et al. & - & - & - & - & - & - & - & - & - & - & - & $\begin{array}{l}\text { PC -based, EHR- } \\
\text { integrated }\end{array}$ \\
\hline Linder et al. & - & - & + & - & + & - & - & - & - & - & - & $\begin{array}{l}\text { PC -based, EHR- } \\
\text { integrated }\end{array}$ \\
\hline Litvin et al. & - & - & - & + & - & - & - & - & + & + & - & $\begin{array}{l}\text { PC -based, EHR- } \\
\text { integrated }\end{array}$ \\
\hline Mainous et al. & - & - & - & + & - & - & - & - & - & - & - & $\begin{array}{l}\text { PC -based, EHR- } \\
\text { integrated }\end{array}$ \\
\hline McGinn et al. & - & - & - & + & + & - & - & - & - & - & - & $\begin{array}{l}\text { PC -based, EHR- } \\
\text { integrated }\end{array}$ \\
\hline $\begin{array}{l}\text { Nachtigall et } \\
\text { al. }\end{array}$ & - & - & - & + & - & - & - & - & - & - & + & PC -based \\
\hline Rattinger et al. & - & - & - & - & - & - & - & - & - & - & - & $\begin{array}{l}\text { PC -based, EHR- } \\
\text { integrated }\end{array}$ \\
\hline Rubin et al. & + & + & - & - & - & - & - & - & - & - & - & $\begin{array}{l}\text { Stand-alone, PDA- } \\
\text { based }\end{array}$ \\
\hline Samore et al. & + & + & - & - & - & - & - & - & - & - & - & $\begin{array}{l}\text { Paper-based or PDA- } \\
\text { based }\end{array}$ \\
\hline Tafelski et al. & - & - & - & + & - & - & - & - & - & - & + & PC -based \\
\hline Vincent et al. & - & - & - & - & - & - & - & - & - & - & - & $\begin{array}{l}\text { PC -based, CPOE- } \\
\text { integrated }\end{array}$ \\
\hline
\end{tabular}

PC: Personal Computer, EHR: Electronic Health Record, EMR: Electronic Medical Record, CPOE: Computerized Physician Order Entry system, PDA: Personal Digital Assistant. 


\subsection{How is the effect of the CDSSs for ASPs measured?}

Primary aim of this scoping review is not to evaluate how effective or successful the studied CDSSs for ASPs are. Rather, we strive to explore and gain more insight into the aspects of support itself. Therefore, we summarize what kind of outcome measures are taken into account in the evaluation of the CDSSs. The systems aim at optimally supporting an ASP. According to McGowan [48], the effects of an ASP can be measured using four outcome variables: patient outcomes, patient safety, resistance and cost. Patient outcomes refers to "all of the clinically justifiable outcomes of stewardship", e.g., optimized antimicrobial therapy. Patient safety concerns all kinds of adverse effects, e.g., mortality rates, complications, toxicity, super-infections. Resistance relates to the effects of an ASP that go beyond the individual patient and is about the preservation of antimicrobials so they remain successful in the future. Finally, ASP interventions should be the most cost-effective option (i.e., the best ratio of outcome versus costs), compared to other interventions or baseline practice [49].

Besides the above-mentioned outcome variables, we propose to add a fifth outcome variable, based on previously-published data [17, 46, 50]: behavioral outcomes, e.g., physicians' adherence (a.k.a. 'compliance') to the advice given by the CDSS. The purpose of including behavioral outcomes is to gain insight in to what extent the effects may correlate with the use of a given CDSS. After all, if the CDSS is not being used, or if its advice is not adhered to, any effects are probably not related to its use.

We screened studies for whether they have evaluated the effects of the CDSS on these five variable groups (Table 5). The included articles report on one [28, 34] to six [20, 27] different outcome variables (median 3).

Out of all studied outcome variables, by far the most are patient outcomes [19-21, 23$35,37,39,40]$. All but three $[22,36,38]$ of the included studies have used at least one such outcome. Behavioral outcome variables (e.g., frequency of CDSS use) were also relatively often included [19-22, 24-27, 29-33, 36, 38, 40]. Seven studies did not mention any behavioral outcome variables [23, 28, 32, 34, 35, 37, 39]. Patient safety outcome variables (e.g., mortality) were less often used, a total of ten studies included patient safety outcome variables $[20,23,24,27,31,33,35-37,40]$. Cost outcome variables were evaluated in four of the included studies [20, 22, 32, 33].

From an antimicrobial stewardship point of view, the resistance outcomes are of great importance. However, to measure these, researchers are faced with many challenges (e.g., effects only being found in long term measurements) [49]. Therefore, resistance outcome variables are often not included [48], or poorly reported on [51]. At least in the studies that were included in this review, none considered resistance outcomes. 
Table 5: Description of the outcome variables used to evaluate CDSS success

\begin{tabular}{|c|c|c|c|c|c|c|c|c|c|c|c|c|c|c|}
\hline \multirow[t]{2}{*}{ Author(s) } & \multicolumn{5}{|c|}{ Patient outcomes } & \multicolumn{4}{|c|}{ Patient safety } & \multirow{2}{*}{$\begin{array}{l}\text { Resis- } \\
\text { tance }\end{array}$} & \multirow{2}{*}{$\begin{array}{l}\text { Cost } \\
\text { Cost }\end{array}$} & \multicolumn{3}{|c|}{ Behavioral outcomes } \\
\hline & $\begin{array}{l}A B- \\
\text { use }\end{array}$ & $\begin{array}{l}\text { Test } \\
\text { use }\end{array}$ & ASP & $\begin{array}{c}\text { Diag- } \\
\text { nosis } \\
\text { change }\end{array}$ & $\begin{array}{l}\text { Admis- } \\
\text { sion } \\
\text { rate }\end{array}$ & LOS & Return & $\begin{array}{l}\text { MDRO, } \\
\text { CDI inci- } \\
\text { dence }\end{array}$ & $\begin{array}{c}\text { Morta- } \\
\text { lity }\end{array}$ & & & $\begin{array}{c}\text { CDSS- } \\
\text { use }\end{array}$ & $\begin{array}{l}\text { Satis- } \\
\text { faction }\end{array}$ & $\begin{array}{l}\text { Adhe- } \\
\text { rence }\end{array}$ \\
\hline Agwu et al. & + & - & + & - & - & + & - & - & - & - & + & - & + & - \\
\hline Bourgeois et al. & + & - & + & - & - & - & - & - & - & - & - & + & + & - \\
\hline Buising et al. & + & - & + & - & - & + & - & - & + & - & + & - & - & + \\
\hline Carman et al. & - & + & + & - & - & - & - & - & - & - & - & + & + & + \\
\hline Charani et al. & - & - & - & - & - & - & - & - & - & - & + & + & + & - \\
\hline Chow et al. & + & - & - & - & - & - & + & + & + & - & - & - & - & - \\
\hline Dean et al. & + & - & + & - & + & + & - & - & + & - & - & - & - & - \\
\hline Demonchy et al. & - & - & + & + & - & - & - & - & - & - & - & - & - & - \\
\hline Filice et al. & - & - & + & - & - & - & - & - & + & - & - & - & - & - \\
\hline Hingorani et al. & - & - & - & - & - & - & + & - & - & - & - & + & - & + \\
\hline Hulgan et al. & - & - & + & - & - & - & - & - & - & - & + & - & - & - \\
\hline Hum et al. & - & - & - & - & - & - & - & - & - & - & - & + & + & - \\
\hline Linder et al. & - & - & + & - & - & - & + & - & - & - & - & + & - & - \\
\hline Litvin et al. & - & - & + & - & - & - & - & - & - & - & - & + & - & - \\
\hline Mainous et al. & + & - & + & - & - & - & - & - & - & - & - & + & - & - \\
\hline McGinn et al. & + & + & + & - & - & - & + & - & - & - & - & + & - & + \\
\hline Nachtigall et al. & + & - & + & - & - & - & - & - & + & - & - & - & - & + \\
\hline Rattinger et al. & - & - & + & - & - & - & - & - & - & - & - & - & - & - \\
\hline Rubin et al. & - & + & + & - & - & - & - & - & - & - & - & + & + & + \\
\hline Samore et al. & + & - & + & - & - & - & - & - & - & - & - & + & - & - \\
\hline Tafelski et al. & + & + & + & - & - & - & - & - & + & - & - & - & - & + \\
\hline Vincent et al. & - & - & + & - & - & - & - & - & - & - & - & - & - & - \\
\hline
\end{tabular}

AB: Antibiotic, ASP: Antibiotic Stewardship Program, LOS: Length of Stay, CDSS: Clinical Decision Support System, MDRO: Multi Drug Resistant Organism, CDI: Clostridium Difficile Infection. 


\section{Discussion}

Aim of this scoping review was to provide insight in key factors, or a guideline for, the development of usable and user-friendly CDSSs for ASP. Therefore, the current study focused on the research question:

How user-centered and supportive are Clinical Decision Support Systems for Antimicrobial Stewardship in practice?

First step in being able to improve current practice, is knowing what current practice is. As mentioned before, in our view, both the user-centeredness and user-friendliness of eHealth technology are key to its success [7-10, 17, 50]. Based on the current scoping literature review, we must conclude that user-centeredness of CDSSs is often overlooked. Or, if not overlooked, the amount of attention that is paid to the user-centeredness often remains limited, due to the (myopic) clinical viewpoint of the studies. The same goes for the supportiveness of the CDSSs. Although some studies did measure behavioral outcomes such as CDSS use or adherence, the focus on what was done to make the CDSSs as supportive as possible, often lacks.

Rather than prescribing how a CDSS should be defined, how it should offer support, we propose to formulate a framework for the development, implementation, evaluation of and reporting on CDSSs for ASP. By such a framework, we hope that the field of CDSSs could progress, since it allows for a more rigorous evaluation of the strengths and weaknesses of the systems, and a better comparison of tools.

Wright and Sittig [15], in their paper, already proposed a method for the evaluation of CDS architectures. They represent a technical approach to evaluation, e.g., focusing on features which a CDSSs should possess and how it can be integrated in other systems.

While such method is highly useful and desirable, we feel that it might (or should) be complemented with a framework to evaluate the user-side of CDSSs. For example, Wright \& Sittig mention that it is important that "content (of a CDSS, eds.) is integrated into workflow". We strongly believe this has value for work flow integration, but to achieve this with CDSS the user-perspective should be taken into account. The preconditions from the user-perspective, that we found via this scoping literature review, are discussed below.

\section{Description of CDSSs should be standardized}

In the included studies, hardly any explicit definition of a CDSS was reported. Also, the way the studied CDSSs were described, varied strongly. To improve and standardize the evaluation and reporting of CDSSs, we propose to use a standardized framework for the description of all CDSSs. For this purpose, the model by Wright \& Sittig can be applied. Additionally, the CONSORT-EHEALTH checklist can be used, offering a guideline on what information should be described and how this can be done [52].

\section{Potential end-users should be involved in development}

The development of the studied CDSSs for ASP received relatively little attention. If something was mentioned, it was noticeable that their development was policy- or expertdriven. The fit between eHealth technology and clinical practice is of paramount importance for its success. Involving end-users throughout the development process of eHealth technology has shown to facilitate such a fit between user, use context and 
technology $[9,53]$. However, potential end-users were hardly involved in the development process. This is unfortunate, since it has been shown that adherence and the fit with clinical practice can be improved by closely cooperating with end-users during every stage of development $[17,53]$. This was also recognized in one of the included studies, where it was stated that "the sustained improvement in guideline adherence observed in our study may be due in part to the process of developing the CDSS, which actively involved ward physicians as a target user group" (pp. 7) [40].

\section{The look and feel of the CDSS should explicitly be considered}

Design choices that were made, were hardly made explicit. For the articles that did provide some information about the design of their CDSS, only a few mention the involvement of a designer (to plan the look and feel of the CDSS), or even making deliberate design choices. Moreover, it was hardly ever indicated whether these choices, or rather, the design as a whole, had been evaluated with (potential) end-users. Yet, the importance of doing so is not only subscribed by our own research in the field of eHealth technology in general $[9,50,53,54]$, but also by Thursky \& Mahemoff [14] who specifically focused on CDSSs.

\section{A CDSS for ASP should offer support throughout the care process}

An ASP consists of several stages in the care process: performing lab tests, setting a diagnosis and prescribing antimicrobials. Most of the studied CDSSs support several stages or even the full process of ASP. However, some studies only offer support at one stage of ASP. Ideally, a CDSS should offer support in all of these stages so that the greatest impact can be achieved. This would fit an integrated stewardship model [44] and would make optimal use of the added value and benefits of technology.

\section{Implementation activities are pivotal for CDSS success}

We found that most of the included studies describe the activities they have undertaken for successful implementation of the CDSS. Even more so, most of them applied multi-faceted implementation interventions, e.g., a combination of education and training. Multi-faceted implementation strategies are preferable over single-faceted strategies, as reported previously [46].

However, we want to emphasize that successful implementation is not something that is done at a single time point. Rather, it is a process that starts during development and continues for as long as the technology is being used in practice. In the medical field, knowledge progresses fast and healthcare practice must always keep up with it. This requires frequent updates for the content of eHealth technology that is used. Maintenance of eHealth technology (such as CDSSs) in order to continuously keep it up to date, is an essential but tricky part of successful long-term implementation [55].

\section{Evaluation of the effects of CDSSs should be holistic and robust}

Not only the outcome variables which were used in the included studies varied, also the methodology and study design were diverse. The studies used very different units of measurement (i.e., physicians, patients, encounters). This makes it difficult to compare the success of different CDSSs. Furthermore, sometimes an ASP with a CDSS is compared to an ASP without CDSS, while sometimes an ASP that includes a CDSS is compared with no ASP at all. This makes comparing the success of CDSSs for ASP rather difficult, since moderating variables may have great influence. 
It is not only important to standardize reporting on CDSSs, but the evaluation itself should be approached in a more rigorous and holistic way. More emphasis should be placed on thoroughly understanding the effects of CDSSs, instead of 'simply' comparing clinical outcome variables. Data on CDSS use are essential since this will give an indication whether any found effects could be attributed to the CDSS. Suboptimal adherence is a known barrier for success of CDSSs. Therefore, more emphasis must be placed on the evaluation of behavioral outcomes (such as actual use), to gain more insight into what aspects of a CDSS lead to optimal adherence levels, and thereby may have contributed to the found effects [17]. Log-data analysis has already shown to be an excellent method to gain more insight into how technology is being used [55-58].

\subsection{Limitations of the current study}

Inherent to choosing to perform a scoping literature review, our study may suffer from some limitations. We used strict inclusion and exclusion criteria. This also meant that some potentially relevant articles may have been missed if they did not use the keywords that were used in the search query in keywords, title or abstract. However, using a scoped search strategy allowed us to investigate a rather broad field of study (CDSSs for ASP) with a rather specific focus (user-friendliness and usability of such systems). Also, due to the nature of this study, we did not evaluate the quality of the included studies. However, we believe that this would not be of added value for the current study aim, i.e., gaining insight into the field of study of CDSSs for ASP.

\section{Conclusion}

Aim of Clinical Decision Support Systems (CDSSs) is to offer clinicians with the support they need in daily clinical practice. However, to what extent they are actually supportive remains unclear. Although prior research has demonstrated that in order to achieve clinical success, it is paramount that CDSSs are easy to use, few of the included studies pay attention to the usability and user-friendliness of the CDSSs. We propose expanding an existing framework by Wright \& Sittig, for evaluation of CDS architectures, adding crucial element from the user-perspective, covering the usability and user-friendliness of CDSSs. 


\section{Authors' contributions}

NBdJ was involved in all phases of the study. LvGP and JW were involved in study design. JW was involved as a second reviewer in the scoped literature review. JWD and BS contributed as second reviewers in the evaluation of the outcome measures. NBdJ drafted the manuscript. All authors drafted and revised the manuscript, and all authors read and approved the final manuscript.

\section{Acknowledgements}

This project was funded by the Interreg IVa "EurSafety Health-net" project. The funding source had no involvement in study design, execution or reporting. We would like to thank E. Baluch, MSc. for her work in exploring the possibilities of performing a scoping literature review in this field of study.

\section{Competing interests}

The authors declare that they have no competing interests. 


\section{References}

1. World Health Organization. The evolving threat of antimicrobial resistance - options for action. Geneva, Switzerland: WHO Press; 2012.

2. Fishman, N., Antimicrobial stewardship, The American Journal of Medicine, 2006, 119(6, Supplement 1), p. S53-S61.

3. Gonzales, R., Malone, D.C., Maselli, J.H., et al., Excessive antibiotic use for acute respiratory infections in the united states, Clinical Infectious Diseases, 2001, 33(6), p. 757-762.

4. Van Houten, M.A., Luinge, K., Laseur, M., et al., Antibiotic utilisation for hospitalised paediatric patients, International Journal of Antimicrobial Agents, 1998, 10(2), p. 161 164.

5. Owens, R.C., Ambrose, P.G., Antimicrobial stewardship and the role of pharmacokinetics-pharmacodynamics in the modern antibiotic era, Diagnostic Microbiology and Infectious Disease, 2007, 57(3), p. S77-S83.

6. Davey, P., Brown, E., Charani, E., et al., Interventions to improve antibiotic prescribing practices for hospital inpatients, Cochrane Database Syst Rev, 2013, 4(4).

7. Wentzel, M.J., Keeping an eye on the context: Participatory development of ehealth to support clinical practice, 2015, University of Twente, Enschede.

8. Van Gemert-Pijnen, J.E.W.C., Peters, O., Ossebaard, H.C., Improving eHealth, Eleven International Publications; 2013.

9. Wentzel, J., Van Velsen, L., Van Limburg, M., et al., Participatory ehealth development to support nurses in antimicrobial stewardship, BMC Medical Informatics and Decision Making, 2014, 14(45).

10. Verhoeven, F., When staff handle staph: User-driven versus expert-driven communication of infection control guidelines, 2009, University of Twente, Enschede.

11. Hunt, D.L., Haynes, R.B., Hanna, S.E., et al., Effects of computer-based clinical decision support systems on physician performance and patient outcomes: A systematic review, Jama, 1998, 280(15), p. 1339-1346.

12. Wright, A., Sittig, D.F., A four-phase model of the evolution of clinical decision support architectures, International Journal of Medical Informatics, 2008, 77(10), p. 641-649.

13. Kawamoto, K., Houlihan, C.A., Balas, E.A., et al., Improving clinical practice using clinical decision support systems: A systematic review of trials to identify features critical to success, BMJ, 2005, 330(7494), p. 765.

14. Thursky, K.A., Mahemoff, M., User-centered design techniques for a computerised antibiotic decision support system in an intensive care unit, International Journal of Medical Informatics, 2007, 76(10), p. 760-768.

15. Wright, A., Sittig, D.F., A framework and model for evaluating clinical decision support architectures, Journal of Biomedical Informatics, 2008, 41(6), p. 982-990.

16. Kannry, J., McCullagh, L., Kushniruk, A., et al., A framework for usable and effective clinical decision support: Experience from the icpr randomized clinical trial, eGEMS, 2015, 3(2), p. 1150.

17. Van Gemert-Pijnen, J.E.W.C., Niiland, N., Van Limburg, M., et al., A holistic framework to improve the uptake and impact of eHealth technologies, Journal of Medical Internet Research, 2011, 13(4), p. el11.

18. Arksey, H., O'Malley, L., Scoping studies: Towards a methodological framework, International Journal of Social Research Methodology, 2005, 8(1), p. 19-32.

19. Bourgeois, F.C., Linder, J., Johnson, S.A., et al., Impact of a computerized template on antibiotic prescribing for acute respiratory infections in children and adolescents, Clinical Pediatrics, 2010, 49(10), p. 976-983.

20. Buising, K.L., Thursky, K.A., Black, J.F., et al., Improving antibiotic prescribing for adults with community acquired pneumonia: Does a computerised decision support system 
achieve more than academic detailing alone? - a time series analysis, BMC Medical Informatics and Decision Making, 2008, 8(1).

21. Carman, M.J., Phipps, J., Raley, J., et al., Use of a clinical decision support tool to improve guideline adherence for the treatment of methicillin-resistant staphylococcus aureus: Skin and soft tissue infections, Advanced Emergency Nursing Journal, 2011, 33(3), p. 252-266.

22. Charani, E., Kyratsis, Y., Lawson, W., et al., An analysis of the development and implementation of a smartphone application for the delivery of antimicrobial prescribing policy: Lessons learnt, Journal of Antimicrobial Chemotherapy, 2013, 68(4), p. 960967.

23. Filice, G.A., Drekonia, D.M., Thurn, J.R., et al., Use of a computer decision support system and antimicrobial therapy appropriateness, Infection Control and Hospital Epidemiology, 2013, 34(6), p. 558-565.

24. Linder, J.A., Schnipper, J.L., Tsurikova, R., et al., Documentation-based clinical decision support to improve antibiotic prescribing for acute respiratory infections in primary care: A cluster randomised controlled trial, Informatics in Primary Care, 2009, 17(4), p. 231 240.

25. Litvin, C.B., Ornstein, S.M., Wessell, A.M., et al., Use of an electronic health record clinical decision support tool to improve antibiotic prescribing for acute respiratory infections: The abx-trip study, Journal of General Internal Medicine, 2013, 28(6), p. 810-816.

26. Mainous, A.G., Lambourne, C.A., Nietert, P.J., Impact of a clinical decision support system on antibiotic prescribing for acute respiratory infections in primary care: Quasiexperimental trial, Journal of the American Medical Informatics Association, 2013, 20(2), p. 317-324.

27. McGinn, T.G., McCullagh, L., Kannry, J., et al, Efficacy of an evidence-based clinical decision support in primary care practices: A randomized clinical trial, JAMA Internal Medicine, 2013, 173(17), p. 1584-1591.

28. Rattinger, G.B., Mullins, C.D., Zuckerman, I.H., et al., A sustainable strategy to prevent misuse of antibiotics for acute respiratory infections, PLoS ONE, 2012, 7(12), p. e51147.

29. Rubin, M.A., Bateman, K., Donnelly, S., et al., Use of a personal digital assistant for managing antibiotic prescribing for outpatient respiratory tract infections in rural communities, Journal of the American Medical Informatics Association, 2006, 13(6), p. 627-34.

30. Samore, M.H., Bateman, K., Alder, S.C., et al., Clinical decision support and appropriateness of antimicrobial prescribing: A randomized trial, Journal of the American Medical Association, 2005, 294(18), p. 2305-2314.

31. Tafelski, S., Nachtigall, I., Deja, M., et al., Computer-assisted decision support for changing practice in severe sepsis and septic shock, Journal of International Medical Research, 2010, 38(5), p. 1605-1616.

32. Hulgan, T., Rosenbloom, S.T., Hargrove, F., et al., Oral quinolones in hospitalized patients: An evaluation of a computerized decision support intervention, Journal of Internal Medicine, 2004, 256(4), p. 349-57.

33. Agwu, A.L., Lee, C.K.K., Jain, S.K., et al., A world wide web-based antimicrobial stewardship program improves efficiency, communication, and user satisfaction and reduces cost in a tertiary care pediatric medical center, Clinical Infectious Diseases, 2008, 47(6), p. 747-53.

34. Vincent, W.R., Martin, C.A., Winstead, P.S., et al., Effects of a pharmacist-to-dose computerized request on promptness of antimicrobial therapy, Journal of the American Medical Informatics Association, 2009, 16(1), p. 47-53. 
35. Chow, A.L.P., Lye, D.C., Arah, O.A., Mortality benefits of antibiotic computerised decision support system: Modifying effects of age, Scientific Reports, 2015, 5(17346).

36. Hingorani, R., Mahmood, M., Alweis, R., Improving antibiotic adherence in treatment of acute upper respiratory infections: A quality improvement process, Journal of Community Hospital Internal Medicine Perspectives, 2015, 5(3).

37. Dean, N.C., Jones, B.E., Jones, J.P., et al., Impact of an electronic clinical decision support tool for emergency department patients with pneumonia, Annals of Emergency Medicine, 2015, 66(5), p.511-520.

38. Hum, R.S., Cato, K., Sheehan, B., et al., Developing clinical decision support within a commercial electronic health record system to improve antimicrobial prescribing in the neonatal icu, Applied Clinical Informatics, 2014, 5(2), p. 368-387.

39. Demonchy, E., Dufour, J.C., Gaudart, J., et al., Impact of a computerized decision support system on compliance with guidelines on antibiotics prescribed for urinary tract infections in emergency departments: A multicentre prospective before-and-after controlled interventional study, Journal of Antimicrobial Chemotherapy, 2014, 69(10), p. 2857-2863.

40. Nachtigall, I., Tafelski, S., Deja, M., et al., Long-term effect of computer-assisted decision support for antibiotic treatment in critically ill patients: A prospective 'before/after' cohort study, BMJ Open, 2014, 4(12), p. e005370.

41. Linder, J.A., Schnipper, J.L., Tsurikova, R., et al., Documentation-based clinical decision support to improve antibiotic prescribing for acute respiratory infections in primary care: A cluster randomised controlled trial. Informatics in Primary Care, 2009, 4, p. 231-240.

42. Oinas-Kukkonen, H., Harjumaa, M., Persuasive systems design: Key issues, process model, and system features, Communications of the Association for Information Systems, 2009, 24(28), p. 484-501.

43. Oxford University Press; 2014. Designer (def. 1).

44. Dik, J.-W.H., Poelman, R., Friedrich, A.W., et al., An integrated stewardship model: Antimicrobial, infection prevention and diagnostic (AID), Future Microbiology, 2016, $11(1)$, p. 93-102.

45. Van Limburg, M., Van Gemert-Pijnen J.E.W.C., Niiland, N., et al., Why business modeling is crucial in the development of eHealth technologies, Journal of Medical Internet Research, 2011, 13(4), p. e124.

46. Wentzel, J., De Jong, N., Karreman, J., et al., Implementation of MRSA Infection Prevention and Control Measures-What Works in Practice? In: Infection Control Updates. InTech. ISBN 9789535100553.

47. Leung, G.M., Johnston, J.M., Tin, K.Y., et al., Randomised controlled trial of clinical decision support tools to improve learning of evidence based medicine in medical students, BMJ, 2003, 327(7423), p. 1090.

48. McGowan, J.E., Antimicrobial stewardship_the state of the art in 2011: Focus on outcome and methods, Infection Control and Hospital Epidemiology, 2012, 33(4), p. 331-337.

49. Dik, J.-W., Hendrix, R., Poelman, R., et al., Measuring the impact of antimicrobial stewardship programs, Expert Review of Anti-Infective Therapy, 2016, 14 (6), p. 569 575.

50. Verhoeven, F., Steehouder, M.F., Hendrix, R.M.G., et al., Factors affecting health care workers' adoption of a website with infection control guidelines, International Journal of Medical Informatics, 2009, 78(10), p. 663-678.

51. Tacconelli, E., Cataldo, M.A., Paul, M., et al., Strobe-ams: Recommendations to optimise reporting of epidemiological studies on antimicrobial resistance and informing improvement in antimicrobial stewardship, BMJ Open, 2016, 6(2), p. e010134. 
52. Eysenbach G., Consort-eHealth: Improving and standardizing evaluation reports of webbased and mobile health interventions, Journal of Medical Internet Research, 2011, 13(4), p. e126.

53. Beerlage-de Jong, N., Eikelenboom-Boskamp, A., Voss A., et al., Combining usercentered design with the persuasive systems design model; the development process of a web-based registration and monitoring system for healthcare-associated infections in nursing homes, International Journal on Advances in Life Science, 2014, 6(3\&4), p. 262 271.

54. Kelders, S.M., Pots, W.T., Oskam, M.J., et al., Development of a web-based intervention for the indicated prevention of depression, BMC Medical Informatics and Decision Making, 2013, 13(26), p. 1-11.

55. Wentzel, M.J., De Jong, N., Niidam, L., et al., Understanding eHealth use from a persuasive system design perspective: An antibiotic information application for nurses, International Journal on Advances in Life Sciences, 2014, 6(3\&4), p. 210-219.

56. Sieverink, F., Kelders, S.M., Braakman-Jansen, L.M.A., et al., The added value of log file analyses of the use of a personal health record for patients with type 2 diabetes mellitus: Preliminary results, Journal of Diabetes Science and Technology, 2014, 8(2), p. 247 255.

57. Van Gemert-Piinen, J.E.W.C., Kelders, S.M., Bohlmeijer, E.T., Understanding the usage of content in a mental health intervention for depression: An analysis of log data, Journal of Medical Internet Research, 2014, 16(1), p. e27.

58. Kelders, S.M., Van Gemert-Pijnen, J.E.W.C., Using log-data as a starting point to make eHealth more persuasive, In: S. Berkovsky, J. Freyne, eds, Persuasive Technology: Springer Berlin Heidelberg, 2013, p. 99-109. 


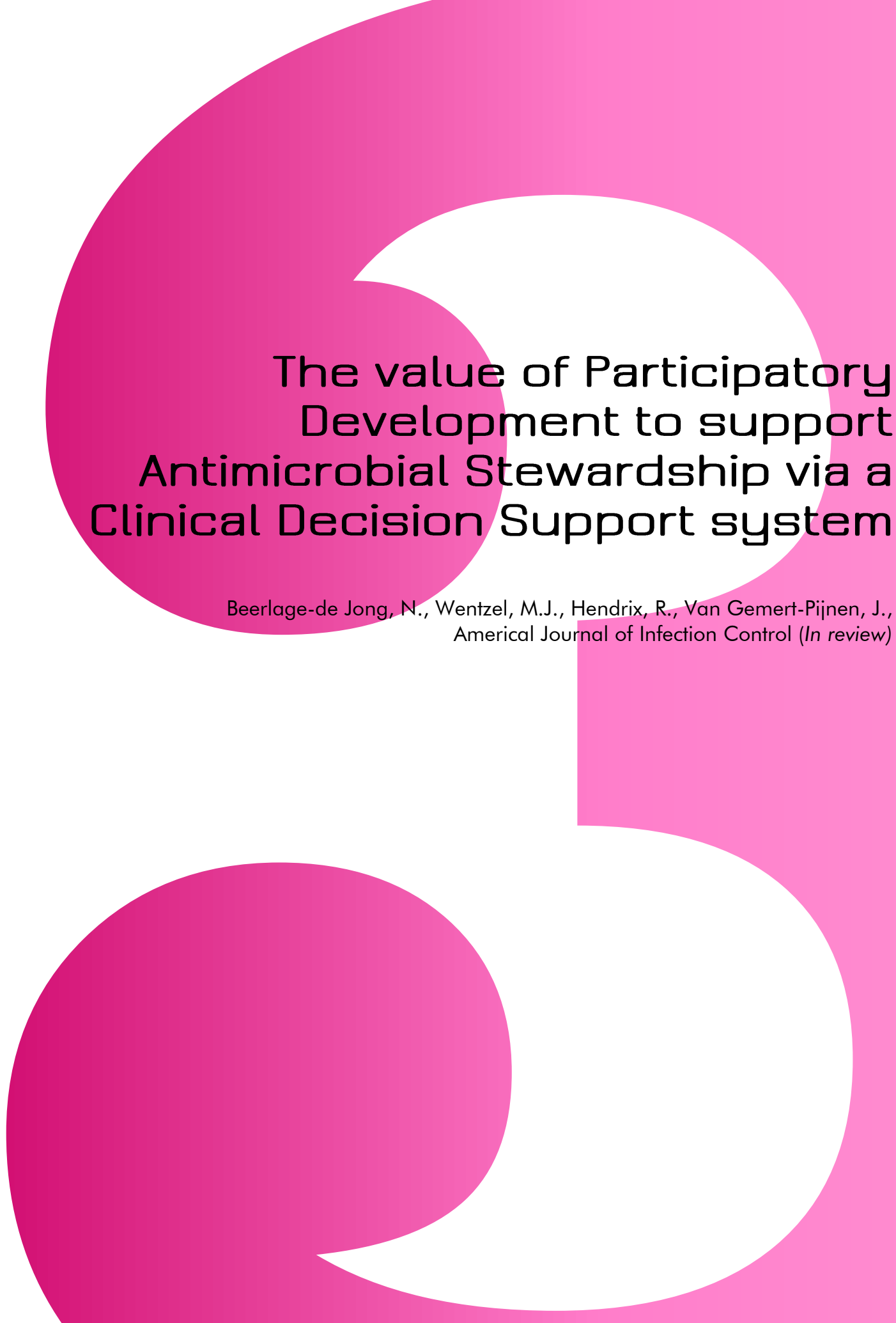


\section{Abstract}

Current Clinical Decision Support Systems (CDSSs) for Antimicrobial Stewardship Programs (ASP) are guideline- or expert-driven. They are focused on (clinical) content, not on supporting real time workflow. Thus, CDSSs fail to optimally support prudent antimicrobial prescribing in daily practice. The aim of this paper is to demonstrate why and how participatory development (involving end-users and other stakeholders) can contribute to the success of CDSSs in ASP.

A mixed-methods approach was applied, combining scenario-based prototype evaluations (to support verbalization of work processes and out of the box thinking) among six medical residents with an online questionnaire (to cross-reference findings of the prototype evaluations) among 54 Dutch physicians.

The prototype evaluations rendered insight into the end-users and their way of working, but also in their needs and expectations. The online questionnaire among a larger group of medical specialists, but also among lung- and infection experts, complemented the findings of the prototype evaluations.

Low-fidelity prototypes of a technology allow researchers to get to know the end-users, their way of working and their work context. Involving experts allows technology developers to continuously check the fit between technology and clinical practice. The combination of both enables the participatory development of technology to successfully support ASP. 


\section{Introduction}

Clinical Decision Support Systems (CDSSs) are a relatively well-studied and widely used form of eHealth technology to support physicians in prudently prescribing antimicrobials [1]. This makes these systems a valuable asset for an Antimicrobial Stewardship Program (ASP). An ASP, a multilevel program based on a "theragnostic" approach [2], is intended to deal with the global health problem of infections with multi-drug resistant organisms, by guiding prudent use of antimicrobials based on adequate diagnostics [2, 3]. However, despite promising results [4], the implementation of such programs still remains challenging [5]. Implemented systems are not always used or adhered to [6, 7].

A recently performed scoping review [1] has dug deeper into this phenomenon. It has shown that end-users were hardly involved in the development of the CDSSs. This means that it is very likely that CDSSs are successful at reflecting clinical standards, but fail to support their users in practice [1]. It has been suggested that this contributes to the low use and adherence rates [6, 8]. Increasingly, studies show a need for a behavioral and/or social approach to the development of interventions in ASP [9, 10]. This can be done by adopting a participatory development approach (involving end-users and other stakeholders).

Via the description of the development process of a CDSS for ASP, the current paper aims to demonstrate why a participatory development approach renders important insights to develop successful ASP-supporting technology. In addition, we describe what kind of information it yields that contributes to the success of the technology. This is done by applying a mixed methods design to (I) gain insight into the potential end-users' perceived needs, and (II) cross-referencing those perceived needs with actual needs. Perceived needs are evaluated via scenario-based prototype evaluations among medical residents, with a low-fidelity prototype of a CDSS for ASP. Actual needs are evaluated via an online questionnaire among a larger group of physicians. The questionnaire focuses on clinical practice of a specific aspect of ASP (diagnostic tests and parameters) that is often overlooked, but is - in our view - one of the pillars of ASPs [2].

\section{Methods}

\subsection{Scenario-based prototype evaluations}

Gaining insight into the perceived needs of potential end-users of a technology can be more difficult than it seems. Merely inquiring about their needs, without offering some concrete example of what the technology might look like, requires great imagination. In this part of the study, physicians who work in practice are presented with an example of what a CDSS might look like. In addition, they are given a real-life scenario. This is a description of a situation that they could encounter in clinical practice. Offering a scenario triggers participants to think about how they make clinical decisions. Also, it makes it easier for them to imagine what it would be like to work with the technology in clinical practice [11]. Thus participants can visualize and verbalize their perceived needs, while researchers gain insight in the end-users' work- and think-processes.

\subsubsection{Participants}

The prototype evaluations took place at an 800-bed teaching hospital. Participants were selected via convenience and snowball sampling and were invited via e-mail. Six medical 
residents (four female) were invited and participated in the study. Their average age was 26.7 years (26-28 years), with working experience of on average 11.7 months (4-22 months), at different departments (i.e., surgery, gastroenterology, cardiology, pediatrics and neurology).

\subsubsection{Procedure and materials}

First, participants were asked about their background and work processes, to gain insight in the context within which the results should be interpreted. Specifically, questions focused on experience with prescribing antimicrobials and on support systems that are currently being used for that.

Then, participants were presented with a low-fidelity prototype of a CDSS. The prototype of a CDSSs to support the prudent prescribing of antimicrobials was created based on what we had learned from prior research about existing CDSSs [1]. The prototype (see Figure 1) was created using Balsamiq software, allowing the physicians to click through the screens of the prototype. To guide them in doing so, a real-life scenario was developed in cooperation with a clinical microbiologist:

A patient is referred to you by his General Practitioner. He has a high fever and an increased respiratory rate. You suspect that the patient has pneumonia.

Participants are asked what kind of support they would like to receive in the given situation and why. The individual prototype evaluations took about 45 minutes each. With permission of the participants, all prototype evaluations were audio-recorded.
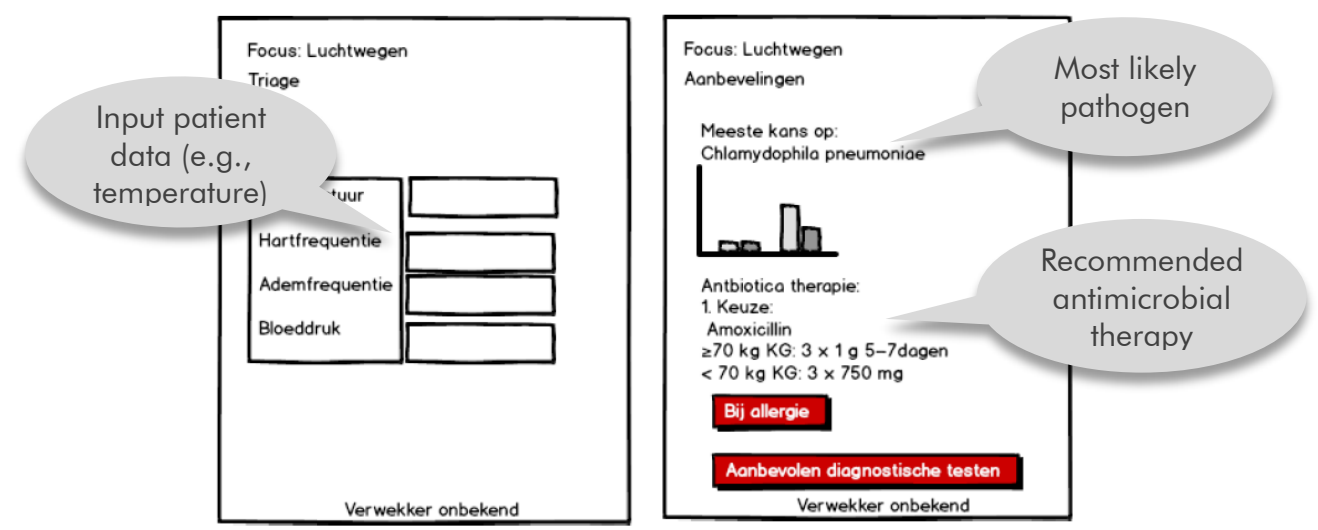

Figure 1. Example screens of prototype (in Dutch) of a CDSS for antimicrobial prescribing

\subsubsection{Data-analysis}

Verbatim transcripts of the audio-recordings were analyzed using a codebook. To do so, the coder (NBdJ) read and reread all transcripts, to familiarize herself with the data. Based on these data a codebook was developed. It contained codes related to the various topics of interest, i.e., experience with support systems, need for a CDSS, type of support needed and the evaluation of the low-fidelity prototype CDSS. Two researchers (NBdJ and JW) independently coded $10 \%$ of the dataset to check the reliability of the codebook. This resulted in a Cohen's Kappa of 0.807 , indicating very good reliability. For data-analysis, the codes were grouped and quotes were summarized. Descriptive statistics were calculated for all codes. 


\subsection{Online questionnaire to gain insight in current ASP practice}

An online questionnaire was used to cross-reference some of the findings of the prototype evaluations. This is done (1) to disclose potential "say/do problems" [12]; (2) to investigate the use context and prototype's fit with it; but also (3) to show why one should not only ask end-users what they think they need, but should also validate this with practice and other stakeholders (in this example: specialists in the field of the given case -lung disease- or in the field of infections and/or antimicrobial prescribing).

The current paper does not intent to fully and into detail describe the development of a CDSS, but rather to illustrate some of the challenges and possible ways to deal with them. Therefore, we zoom in on a single type of support (i.e., the selection of appropriate diagnostic tests) of which we know from literature that it is first and foremost necessary [2], but also available [1], and from scenario-based prototype evaluations that the perceived need for this kind of support is limited. The aim of the questionnaire is to investigate whether this kind of support should be a need. We assume that for such support not to be needed, it would require representatives from clinical practice and experts to agree on a clear vision about what actions should be taken. In this case, that means they should consent on what diagnostic tests should be used for a given scenario. The scenario matched the one that was used in user testing.

\subsubsection{Participants}

The scenario-based user tests demonstrated that one of the most important reasons for experiencing a need for support was that clinicians often treat patients with infections caused by pathogens outside of their own medical specialty. Thus, it was decided send email invitations to clinicians from any kind of medical specialty to participate in the online questionnaire, via hospital clinical microbiologists. A total of 75 physicians started filling out the online questionnaire, 54 of whom actually completed it. These 54 physicians (33 male) represent 26 different medical specialties (e.g., pediatrics, medical microbiology, cardiology, gynecology, internal medicine). Their mean age was 46.3 years (ranging from 32-64 years), with on average 12.7 years of work experience (range $<1-32$ years).

\subsubsection{Procedure and materials}

The real-life scenario that was used in the prototype evaluation was also used in the online questionnaire. It aided the participants in determining what diagnostic tests they considered important. Physicians were asked to indicate (on a 5-point Likert scale) how important they consider different diagnostic parameters and tests to be. For that purpose, they were presented with a list of possibilities: 
- Temperature

- CRP

- Venous oxygen saturation

- Heart rate

- Procalcitonin

- Creatinine

- Blood pressure

- Leukocyte count

- Urea test

- Respiratory rate
- Differentiation

- Ultrasound abdomen

- Blood culture

- BSE

- Lumbar Puncture

- Sputum culture

- X-thorax

- Urine culture

- X-abdomen

- Feces culture
- Rapid test for Pneumococcal colonization

- Clostridium rapid test

- Intravenous pyelogram

- Urine sediment

- Lactate

- Other

This list was developed in cooperation with a clinical microbiologist, and was based on scientific literature and medical guidelines [13-15]. The potentially relevant parameters were complemented with less relevant parameters, to provide additional options. Questions were included to gain insight into demographic characteristics of the participants, and their motivation for their rating of the diagnostic parameters and tests.

\subsubsection{Data-analysis}

IBM SPSS Statistics 20.0 was used to analyze data. The frequencies with which physicians indicated a certain parameter or test was (very) important, neutrally important or (very) unimportant were analyzed using descriptive statistics. The aim of this part of the study was to measure consensus. In existing literature, different thresholds for consensus are applied [16, 17]. In striving towards strong consensus, the rather conservative threshold of $80 \%$ agreement was set for the current study.

\section{Results}

\subsection{Scenario-based user tests}

\subsubsection{Current clinical practice}

All six participating medical residents indicated having experience with prescribing antimicrobials. Additionally, they all have experience with using some kind of support system to support them in doing so. Most of the systems that participants report to be working with are digital support systems (e.g., online guidelines of the Dutch Working Party on Antibiotic Policy). Inter-personal support systems (e.g., consultations with medicalmicrobiologists or other medical specialists) and paper-based support systems (e.g., hospital protocols) are less often mentioned.

Quote 1. "For more complicated cases [...] we regularly consult a medicalmicrobiologist. Because it is his everyday work, he knows exactly what should be used for what, and has all the test results at hand." surgery, Male

Mostly, patients are initially treated with a broad spectrum antibiotic. Some participants pro-actively explained the triggers to start any antimicrobial therapy. Triggers that were mentioned were: anamnesis, the patient's history (i.e., prior infections, prior lab test results, prior healthcare institution stay, recent time spend abroad, recent stays in an environment with a risk of extraordinary bacteria), estimation of severity/mortality scores, 
most likely pathogen, lung imaging and whether infection is community- or hospitalacquired.

Quote 2. "And whether the patient went to a sauna or something like that, because of which he would be at risk for other bacteria that the ones that you would find in normal open air. Those are things you keep in mind."

Gastroenterology, female

Ideally, upon initiating an antimicrobial therapy, diagnostic tests should be requested. Although not explicitly asked, five of the respondents pro-actively indicated what kind of diagnostic tests they would request. Diagnostic tests that they mentioned were sputum culture, blood culture, lung imaging, temperature, blood pressure, heart frequency. Some tests were each mentioned by one participant: respiratory frequency, anamnesis, physical examination, saturation, Early Warning System (EWS) score (a measure for severity of septic shock), legionella rapid test, leukocyte count and CRP.

One participant (Surgeon, female) mentioned almost all (79\%) of the tests and another one (Gastroenterology, female) also mentioned relatively many tests (57\%). The others each mentioned significantly less (lab) tests (21-29\%).

\subsubsection{Perceived need for a CDSS}

All participants felt that having a CDSS available, would improve their work. However, their reasons for that differ. Participants mention that a CDSS would (1) help them select the right kind, dosage and duration of antimicrobials for rare pathogens or in case of organ (e.g., kidney) failure; (2) save them time, since it automatically integrates a lot of scientific literature into a single advice; (3) be especially helpful for treating patients who have an infection that does not lie within their own specialty; (4) help to avoid unnecessary use of broad spectrum antimicrobials; and (5) help to alert physicians about possible allergies that a patient has, if the CDSS is linked to an Electronic Health Record.

\section{Quote 3. "We encounter all kinds of infections that are not always related to neurology. (...) And basically, we do want to treat all our patients ourselves." \\ Neurology, Male}

On the other hand, barriers were mentioned that would prevent participants from using a CDSS for prescribing antimicrobials. Prerequisites for a CDSS, to deal with perceived barriers are: (1) a CDSS' advice should not be restrictive (2) it should not discard any antimicrobials that would not be harmful if they were used; (3) the participants still want to see alternative possible antimicrobials, so that they can have a final say in which one they select; and (4) the system should not prevent physicians from thinking themselves, because they may come to rely on it too much.

\subsubsection{Perceived system needs}

The CDSS should be offered on smartphone, or a combination of smartphone with personal computer (which is available in the clinic) and tablet. The CDSS should be available 24/7. It would mainly be used during rounds or when starting empiric antimicrobial therapy for newly admitted patients.

\subsubsection{Perceived service needs}

Participants appreciated the fact that the prototype was kept concise. However, some challenges arose concerning its user-friendliness. For example, the term "MDRO" (Multi Drug Resistant Organisms) was unclear, and participants were unfamiliar with the AMBU- 
65 score (a severity index for Pneumonia), preventing them from adequately using part of the prototype.

A CDSS should use and provide references to the reliable guidelines that it was based on, for the users to be able to work with and trust the system. Also, two participants explicitly mentioned that a CDSS should not be restrictive, meaning that is should suggest courses of action, but not compel physicians to do as it says.

Quote 4: "I think you should really see it as guidance and not as something
binding. Of course, protocols are really nice and in many cases you can work
with them but there are also plenty of reasons why you would deviate from
them. (...) there are numerous factors that can change your choice: previous
lab test results, or previous experiences." Surgery, male

Participants indicate that the CDSS should highlight one preferred antimicrobial, and provide others as alternatives, based on degree of illness or other input data. They felt this would help them to quickly select the correct one. Finally, one participant stated that it is necessary for users to have insight in the reasoning - the algorithms - behind the system.

\subsubsection{Perceived content needs}

Existing CDSSs mainly offer decision support on three aspects of prescribing antimicrobials: (1) selecting adequate microbiological laboratory tests, (2) selecting a correct diagnosis, and/or (3) selecting an appropriate antibiotic. Some of the existing CDSSs combine these with 'other' support, e.g., patient information, easy access to scientific literature.

\section{Lab test selection}

Participants mentioned that recommendations of appropriate lab tests might be useful as a reminder for physicians. However, other participants explicitly state that this feature is not needed. This is the only feature of which respondents explicitly mentioned not appreciating or needing it.

Quote 5. "Actually, I think every doctor knows what to do." Gastroenterology, Female

\section{Diagnosis selection}

Participants want a CDSS system to provide the most likely pathogen, and information about whether the infection is most likely community- or hospital-acquired. The prototype version of a CDSS made use of the AMBU-65 score [18] to calculate a severity score for pneumonia. Not all participants were familiar with this specific score, but it was acknowledged as a useful feature for a CDSS.

\section{Antimicrobial selection}

Antimicrobial selection was to most appreciated type of support of the prototype CDSS. Participants saw value in recommendations about the kind of antimicrobial. This also goes for the feature of the prototype where alternative antimicrobial agents were suggested, in case a patient has an allergy.

Quote 6. "That is what makes it difficult: in case of an allergy. That's a practical example that I experienced, it made me realize that I could not find it anywhere." Surgery, Female 
Participants appreciated recommendations about the dosage and duration of the antimicrobial therapy. They mentioned that especially the optimal duration of a therapy is often unclear.

Quote 7. "It is often decided like: 'well, let's make it seven days, or actually, make it ten'. Even though five days might have sufficed." Gastroenterology, Female

Participants also find possible drug-drug interactions and possible side effects useful information. Finally, some aspects of antimicrobial selection support were mentioned by a single participant (i.e., dosage in case of kidney failure, alternative administration routes, when to switch from IV to oral administration and contra-indications).

\section{Other support}

Participants appreciate a link to an information database that was provided within the prototype. It referred to a web-based app for administering antimicrobials in clinical practice that was previously developed for, and greatly appreciated by nurses [19, 20]. They especially liked the combination of a concise CDSS with optional further (in depth) reading in an additional database (see Quote 8 ).

Quote 8. "I do not think you should strive to incorporate everything in this system. That would make it too cluttered. And it remains to be seen whether you always need to know everything. In nine out of ten cases this would suffice." Surgery, Male

\subsection{Online questionnaire}

In the prototype evaluations, participants indicated that they would know what diagnostic tests to perform in the given scenario case. Thus, this kind of support was not needed by some of them.

The participants in the questionnaire study represented many different medical specialties. Among them were experts in the field of infections (i.e., clinical microbiologists, specialists in internal medicine) and experts in the field of the case in the scenario (i.e., lung specialists). The other participating medical specialties do not have prescribing antimicrobials or lung diseases as their core clinical task. It might be that the judgments of 'infection or lung experts' $(n=12)$ and 'other specialties' $(n=42)$ differ. That is investigated in the following paragraphs.

\subsubsection{Expert consensus}

When focusing on the lung or infection experts, ten (40\%) parameters reached consensus: sputum culture, respiratory rate, blood culture, chest radiography, heart rate and blood pressure were all six (24\%) considered (very) important by this sub-group; experts also consented about the unimportance of four (16\%) of the tests: lumbar puncture, ultrasound abdomen, intravenous pyelogram and clostridium rapid test. This automatically means that experts differed in opinion about $60 \%$ (15) of the diagnostic tests (see Figure 2). 


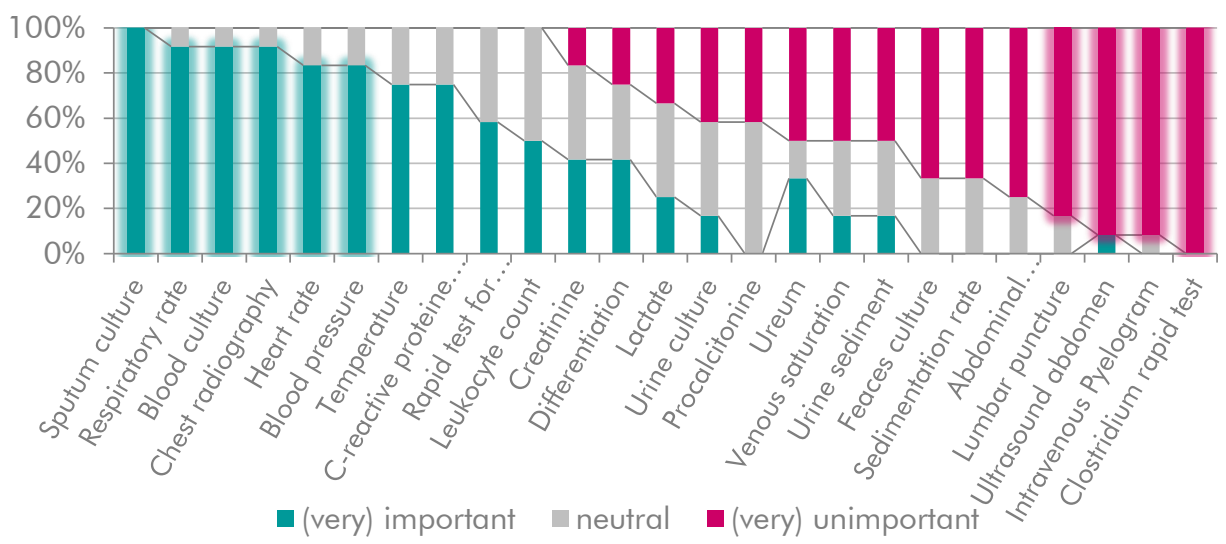

Figure 2. Consensus among lung- and infection specialists

\subsubsection{Consensus among other specialties}

The other medical specialties reached consensus for a total of seven (28\%) of the parameters or tests. One of these $(4 \%)$ was considered important: respiratory rate. The others (24\%) were found (very) unimportant: lumbar puncture, abdominal radiography, ultrasound abdomen, feces culture, Clostridium rapid test and intravenous pyelogram. Thus, their opinions differed for 18 (72\%) of the diagnostic tests (see Figure 3 ).

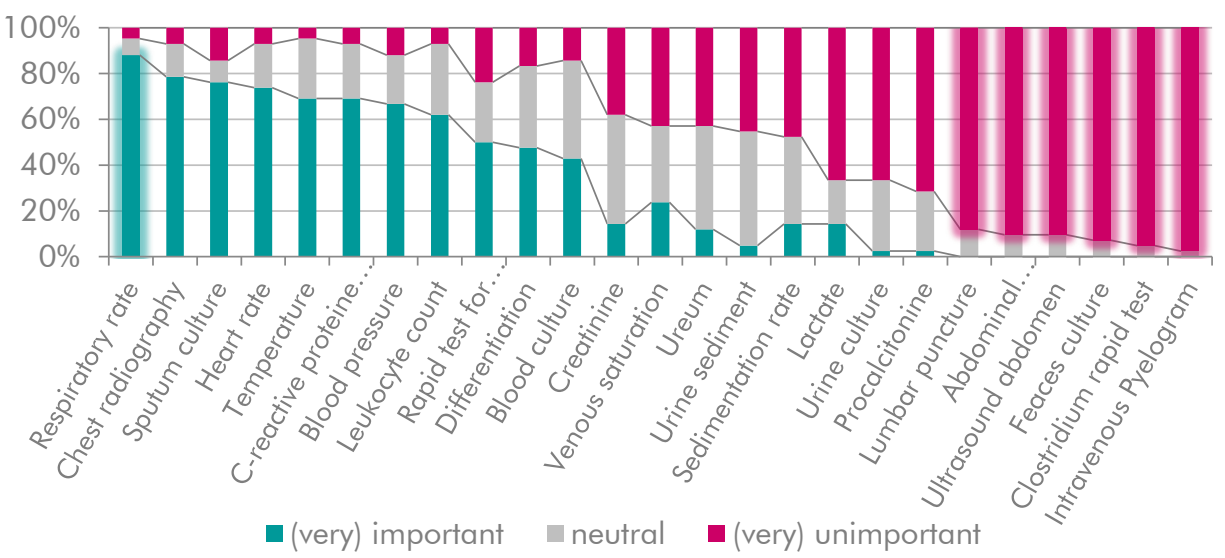

Figure 3. Consensus among other medical specialties

\subsubsection{Comparing lung and infection experts with other medical specialties}

Neither lung and infection experts nor other medical specialties reached consensus very often (both far less than half of the time). Experts did reach consensus more often than the other specialties, but considering the amount of times that no consensus was reached, there is a broad range in preferences or opinions among all medical specialists. Additionally, more than half (59\%) of the times that consensus was reached, a diagnostic test was found unimportant. Rather than agreeing on what should be done in clinical practice, physicians in these cases just agree on what should not or does not have to be done. 


\section{Discussion}

Existing CDSSs for ASP are medically based and clinically focused. They are expert- or guideline driven. Such myopic view leads to CDSSs that do not support the actual processes in patient care and their supportiveness remains limited. At the same time such supportiveness is pivotal to be able to provide 'Stewardship' within an ASP, especially in an integrated stewardship model, with many and complex (intertwined) decisions and considerations to be made. The aim of the current paper is to demonstrate how a participatory development 'Stewardship' can be provided, by developing technology (such as a CDSS) via a participatory development process.

\subsection{Lessons learned}

The main lessons learned, concerning the added value of participatory development of eHealth technology for ASPs, are discussed in this section.

\subsubsection{The technology}

In the current study, end-users and other stakeholders were very much involved. The scenario-based prototype evaluations allowed users to experience what it would be like to work with such a technology and to verbalize clear notions of what the technology should or should not do. Participants were pertinent in stating that it should not be restrictive, as they find it crucial to still be able to use their tacit knowledge (or: 'clinical eye') in treating their patients. This was noted in other studies [21-23], but was also confirmed in the current online questionnaire. The latter helped to surface clear differences in opinions, both among experts and among other medical specialties, about the importance of certain diagnostic tests. The discordance in the opinions of experts is an indication that it is undesirable or even impossible for a CDSS in ASP related diagnostics to provide directive instructions about what to do. Rather, it should provide an instrument to support, motivate and remind physicians to take all the necessary steps for antimicrobial stewardship. This requires for a CDSS to be flexible, to be able to adapt to changing settings and conditions.

\subsubsection{The users}

A previous scoping review [1] demonstrated that CDSSs for ASP generally offers support on one or more aspects of ASP (e.g., selecting lab tests, selecting a diagnosis, selecting an antimicrobial therapy). Of these, the selection of lab tests was least often supported. In the current study, the participatory development approach provided insight in the CDSSs endusers and their support needs. The end-users needs are found on different levels: the need for a CDSS as a whole, but also the need for the different kinds of support such a system can provide. However, 'only' inquiring end-users about their needs does not suffice. This paper has revealed some so called 'say/do' problems [12] and has also provided an example of how researchers can forestall such problems, by including other stakeholders as well. In this case, end-users indicated not appreciating a certain kind of support (selecting diagnostic tests) provided in the CDSS prototype, whereas the online questionnaire demonstrated that this kind of support would never the less be necessary for optimal clinical practice. Experts can and should be involved to cross-reference the perceived needs of end-users, to make sure their needs still fit clinical practice. Nevertheless, some challenges remain: if users do not think they need (a certain kind of) support, then how can we get them to use the technology nevertheless? We suggest, also based on prior research [24], to first of all make sure that using the system is made an integral part of physician's work processes. Thus, the work process itself must be a trigger 
for physicians to use the CDSS. Second, we advocate applying Persuasive Systems Design [25] elements, to persuade users (e.g., via reminders) to use the system at a time that they might not know they need it. In doing so, it is very important to provide reminders scarcely, to prevent 'alert-blindness' in the users [26].

\subsubsection{The context}

In the scoping review [1] hardly any information was found (and if so, information was scarce) about the context within which the CDSSs were to be used. Working with the low fidelity prototype of a CDSS for ASP allowed end-users to clearly indicate how they would like the proposed system to be integrated with existing systems and work processes. They mainly appreciated an integration of the CDSS with Electronic Health Records, since that could save them valuable time with inputting patient data. Additionally, they liked the system to be linked to an information database. This enables the CDSS itself to be kept concise, while its users can still easily and quickly find additional information when necessary.

\subsection{Implications for clinical practice}

For any kind of technology to be able to have a positive impact on clinical practice, it is of paramount importance that technology developers must really gain insight in it. Not only in who is involved in clinical practice, but also e.g., their context, work relations, beliefs, needs, likes and dislikes. This paper offers insights in factors that can contribute to making a CDSS for ASP successful. But more importantly, rather than 'just' providing rules for what a CDSS should be like, it offers a means of revealing such information: via methods for participatory development. The participatory development approach that is described in the current paper can be applied to the development of CDSSs for physicians, but it has also already proven useful for other kinds of technology and other healthcare professionals in ASP [20].

\subsection{Future work}

Based on this and prior research [1, 5, 24, 27-29], we are convinced that the greatest added value of technology in an ASP, lies in creating a 'network' of technologies to support an integral approach to Antimicrobial Stewardship [2]. Adequate and timely diagnostics are the basis for such integrated approach to stewardship, so this should be supported through technology (e.g., via CDSSs). It also requires prudent prescribing of antimicrobials (also supported via e.g., CDSSs), adequate administration and monitoring of antimicrobial therapy (supported via e.g., an information app [19]) and applying infection control measures (supported via e.g., 'plain language' translations of and easy access to protocols and guidelines [28]). Such a network of technologies has a value that is greater than the sum of its parts, as the connection between such technologies allows for a 'smart' system to be developed that draws data from numerous sources (e.g., protocols, patient data). In doing so, it can use big data sets to model and predict outbreaks and provide tailored advice for the treatment of individual patients.

\section{Conclusions}

Participatory development is of great added value for the development of successful ASP. Low-fidelity prototypes of a technology allow researchers to get to know a system's endusers, their way of working and their work context. Additionally, it helps potential end- 
users to visualize what it would be like to work with the system, and to indicate their needs. Involving experts allows technology developers to continuously check the fit between needs and clinical practice. The combination of the both enables the development of technology that is not only user-friendly and successful in terms of uptake, but also is successful in terms of impact and clinical outcomes.

\section{Authors' contributions}

All authors were involved in the design of this study. NBdJ performed data collection. NBdJ and JW performed data analysis. All authors were involved in drafting the manuscript. All authors read and approved the final manuscript.

\section{Acknowledgements}

This project was funded by the Interreg IVa "EurSafety Health-net" project. 


\section{References}

1. Beerlage-de Jong, N., Wentzel, J., Dik, J.-W., et al., Opening the black box of Clinical Decision Support Systems for Antimicrobial Stewardship - A scoping review, International Journal of Medical Informatics, Submitted.

2. Dik, J.-W.H., Poelman, R., Friedrich, A.W., et al., An integrated stewardship model: Antimicrobial, infection prevention and diagnostic (AID), Future Microbiology, 2016, $11(1)$, p. 93-102.9

3. MacDougall, C. and Polk, R.E., Antimicrobial stewardship programs in health care systems, Clinical Microbiology Reviews, 2005, 18(4), p. 638-656.

4. Davey, P., Brown, E., Charani, E., et al., Interventions to improve antibiotic prescribing practices for hospital inpatients, Cochrane Database of Systematic Reviews, 2013, (4).

5. Limburg, A.H.M., Implementing antibiotic stewardship: involving stakeholders in eHealth, 2016, University of Twente: Enschede.

6. Hum, R.S., Cato, K., Sheehan, B., et al., Developing clinical decision support within a commercial electronic health record system to improve antimicrobial prescribing in the neonatal ICU, Applied Clinical Informatics, 2014, 5(2), p. 368-387.

7. Bourgeois, F.C., Linder, J., Johnson, S.A., et al., Impact of a computerized template on antibiotic prescribing for acute respiratory infections in children and adolescents, Clinical Pediatrics, 2010, 49(10), p. 976-983.

8. Nachtigall, I., Tafelski, S., Deja, M., et al., Long-term effect of computer-assisted decision support for antibiotic treatment in critically ill patients: A prospective 'before/after' cohort study, BMJ Open, 2014, 4(12), p. e005370.

9. Hulscher, M.E., Grol, R.P., and Van Der Meer J.W., Antibiotic prescribing in hospitals: a social and behavioural scientific approach, The Lancet Infectious Diseases, 2010, 10(3), p. 167-175.

10. Janes, C.R., Corbett, K.K., Jones, J.H., et al., Emerging infectious diseases: the role of social sciences, The Lancet, 2012, 380(9857), p. 1884-1886.

11. Carroll, J.M., Rosson, M.B., Chin, G., et al., Requirements development in scenariobased design, IEEE Transactions on Software Engineering, 1998, 24(12), p. 1156-1170.

12. Bødker, K., F. Kensing, and J. Simonsen, Participatory IT design: designing for business and workplace realities, 2009: MIT press.

13. Dellinger, R.P., Levy, M.M., Rhodes, A., et al., Surviving Sepsis Campaign: international guidelines for management of severe sepsis and septic shock, 2012, Intensive care medicine, 2013, 39(2), p. 165-228.

14. Müller, F., Christ-Crain, M., Bregenzer, T., et al., Procalcitonin levels predict bacteremia in patients with community-acquired pneumonia: a prospective cohort trial, CHEST Journal, 2010, 138(1), p. 121-129.

15. Watkins, R.R. and Lemonovich, T.L., Diagnosis and management of community-acquired pneumonia in adults, Mortality, 2011, 100, p. 12.

16. Nikolaus, S., Bode, C., Taal, E., et al., Selection of items for a computer-adaptive test to measure fatigue in patients with rheumatoid arthritis: a Delphi approach, Quality of Life Research, 2012, $21(5)$, p. 863-872.

17. Powell, C., The Delphi technique: myths and realities, Journal of advanced nursing, 2003. $41(4)$, p. 376-382.

18. Lim, W., et al., Defining community acquired pneumonia severity on presentation to hospital: an international derivation and validation study, Thorax, 2003, 58(5), p. 377 382.

19. Wentzel, J., Van Velsen, L., Van Limburg, M., et al., Participatory eHealth development to support nurses in antimicrobial stewardship, BMC Medical Informatics and Decision Making, 2014, 14(45). 
20. Wentzel, J., Van Drie-Pierik, R., Nijdam, L., et al., Antibiotic information application offers nurses quick support, American Journal of Infection Control, 2016, 44(6), p. 677 684.

21. Chow, A.L., Lye, D.C., and Arah, O.A., Patient and physician predictors of patient receipt of therapies recommended by a computerized decision support system when initially prescribed broad-spectrum antibiotics: a cohort study, Journal of the American Medical Informatics Association, 2016. 23(e1), p. e58-e70.

22. Chow, A., D.C.B. Lye, and O.A. Arah, Psychosocial determinants of physicians' acceptance of recommendations by antibiotic computerised decision support systems: $A$ mixed methods study, International Journal of Antimicrobial Agents, 2015, 45(3), p. 295-304.

23. Broom, J., Broom, A., Plage, S., et al., Barriers to uptake of antimicrobial advice in a UK hospital: a qualitative study, Journal of Hospital Infection, 2016, 93(4), p. 418-422.

24. Wentzel, M.J., Keeping an eye on the context: participatory development of eHealth to support clinical practice, 2015, University of Twente: Enschede.

25. Oinas-Kukkonen, H., Harjumaa, M., Persuasive systems design: Key issues, process model, and system features, Communications of the Association for Information Systems, $2009,24(28)$, p. 484-501.

26. Kesselheim, A.S., Cresswell, K., Phansalkar, S., et al., Clinical Decision Support Systems could be modified to reduce 'alert fatigue' while still minimizing the risk of litigation, Health Affairs, 2011, 30(12), p. 2310-2317.

27. Gemert-Pijnen, J., Het totstandkomen en functioneren van infectiepreventieprotocollen. Een onderzoek naar communicatie gestuurd door wet-en regelgeving, 2003, University of Twente, Enschede.

28. Verhoeven, F., When staff handle staph: user-driven versus expert-driven communication of infection control guidelines, 2009, University of Twente: Enschede.

29. Beerlage-de Jong, N., Eikelenboom-Boskamp, A., Voss A., et al., Combining usercentered design with the persuasive systems design model; the development process of a web-based registration and monitoring system for healthcare-associated infections in nursing homes, International Journal on Advances in Life Science, 2014, 6(3\&4), p. 262 271. 



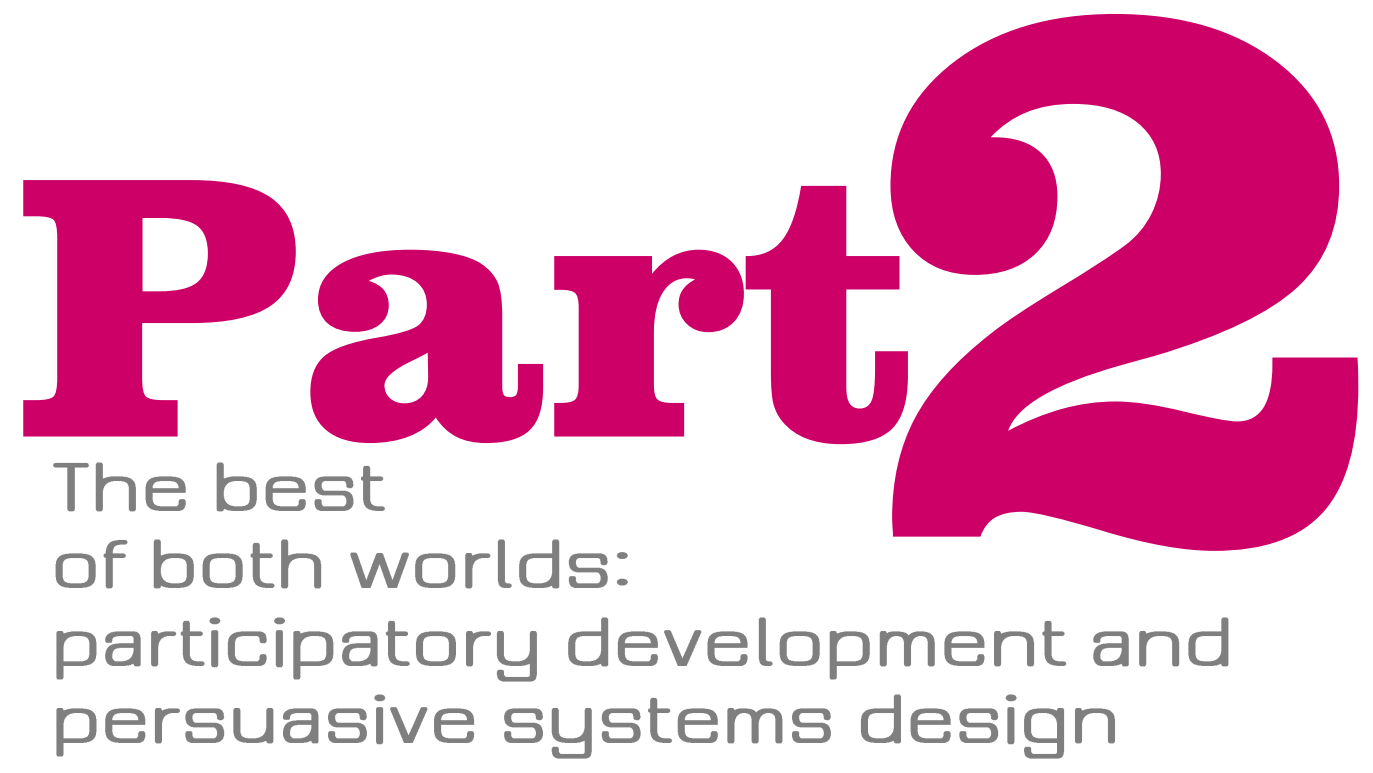




\section{Case}

\section{Measuring and monitoring the prevalence of healthcare- associated infections in nursing homes}

You may not think about it every day (and maybe it is so much the better if you don't, provided that it is not your job to think about it), but it is not only a hospital stay that increases one's risk of acquiring a healthcare-associated infection. Modern day healthcare is dealing with an aging population. This implies that increasingly many people will spend some time of their lives in a long-term care facility (e.g., a nursing home). In such facilities, as in hospital settings, relatively many people live closely together. Also, they often use catheters, IVs (risks for acquiring an infection) or use antimicrobials [1].

It seems clear that the occurrence of healthcare-associated infections (HAls) in nursing homes is just as relevant as their occurrence in hospitals. Yet, whereas up to the ' 60 many studies had been performed about surveillance data for the occurrence of HAls in hospitals $[2,3]$, none have been found to cover nursing homes. It was in 1997 that the Society for Healthcare Epidemiology of America and the Association for Professionals in Infection Control programs (SHEA/APIC) published their position paper, stressing the need for surveillance in long-term care facilities [4].

But what does surveillance even entail? Basically, there are two kinds of surveillance: incidence or prevalence measurements. Incidence stands for the rate of new cases of the disease within a given time period. Thus, this requires constant registration of e.g., infections. Prevalence is the actual number of cases that exist at a certain time point or period. Thus, this requires a single (or a short period of) measurement.

We just mentioned that up to a few decades ago, no surveillance of HAls had been performed in nursing homes. Fortunately, prevalence measures have lately started to be performed in nursing homes as well [5]. These prevalence measures (and the monitoring of its results) allow nursing homes to adjust their (e.g., hygiene) policies as needed. But of course, this will be most effective if they are optimally supported during both registration and monitoring of the data, since registration is a time consuming and complicated task.

For that purpose, the 'Prevalence app' was developed in a cooperation between University of Twente and iPrevent. It applies persuasive design principles to guide elderly care physicians through the registration of their clients, and provides clear feedback of the nursing home's prevalence data. This is complemented with (anonymized) benchmark data of the prevalence data of all participating nursing homes. The development and design process of the Prevalence app is described in Chapter 4. 


\section{References}

1. Centers for Disease Control and Prevention, Antimicrobial resistance surveillance in Europe 2014, in Annual Report of the European Antimicrobial Resistance Surveillance Network (EARS-Net), 2015, ECDC: Stockholm.

2. Barrett, F.F., Casey, J.I., and Finland, M., Infections and antibiotic use among patients at Boston City Hospital, february, 1967, New England Journal of Medicine, 1968, 278(1), p. 5-9.

3. Sherwood, L.M., Parris, E.E., and Feingold, D.S., Hospital-Acquired Infections. New England Journal of Medicine, 1970, 283(25), p. 1384-1391.

4. Smith, P.W. and Rusnak, P.G., Infection prevention and control in the long-term-care facility. Infection Control and Hospital Epidemiology, 1997, 18(12), p. 831-849.

5. Eikelenboom-Boskamp, A., Cox-Blaessens, J.H.M., Boom-Poels, P.G.M., et al., Threeyear prevalence of healthcare-associated infections in Dutch nursing homes, BMC Proceedings, 2011, 5(Suppl 6), p. 158. 



\section{Combining Participatory Development with the Persuasive Systems Design Model}

The Development Process of a Web-Based Registration and Monitoring System for Healthcare-Associated Infections in Nursing Homes

N. Beerlage-de Jong, A. Eikelenboom-Boskamp, A. Voss, R. Sanderman, L. van Gemert-Piinen, International Journal on Advances in Life Science, 2014.

Presented as oral presentation at eTelemed 2014 Barcelona, Spain. 


\section{Abstract}

To improve the usefulness and user-friendliness of eHealth interventions, a framework for the development of eHealth technology has been developed. It combines User-Centered Design with the Persuasive System Design model. The current paper is aimed at offering a (practical) method for the integration of these two design approaches. Via a case study, the paper demonstrates how User-Centered Design and the Persuasive Systems Design model can complement and mutually enrich each other. 


\section{Introduction}

In this paper, we present an extended version of work presented at the Sixth International Conference on eHealth, Telemedicine and Social Medicine (eTELEMED 2014) in Barcelona, Spain [1].

In healthcare, and specifically in infection prevention and-control, many apps have been developed. They are intended to improve quality of care and patient safety, but are definitely not always successful. They often don't fit with the way the intended end-users work or think, or they fulfill a non-existent need. Therefore, the Center for eHealth Research and Disease Management, has developed a framework to develop apps that are successful in daily clinical practice: the CeHRes Roadmap [2]. This framework makes use of User-Centered- Design (UCD) and the Persuasive Systems Design (PSD) model.

Within UCD, during the entire development and design processes of a technology, close cooperation is sought with end-users and other stakeholders. This is done to achieve an optimal fit between the newly developed technology, the context in which it is used and the way the intended end-users work or think [3]. User problems can thus be recognized and prevented. For the prevention of such issues, and to motivate and support the end-users, the PSD model is used [4]. According to this model, technology can be developed with the aim of changing the users' behavior, without using coercion or deception [5][6]. Thus, the technology itself can make a substantial contribution to its own success. We, the Center for eHealth Research and Disease Management, are convinced that the combination of these two models can be of great added value for the development of successful eHealth technology.

This paper describes such a development and design process, with the aim of offering a tool for the development of persuasive and user-friendly eHealth technology. To do so, the different stages of the iterative development process (following the CeHRes Roadmap, see Fig. 1) of a single app is described.

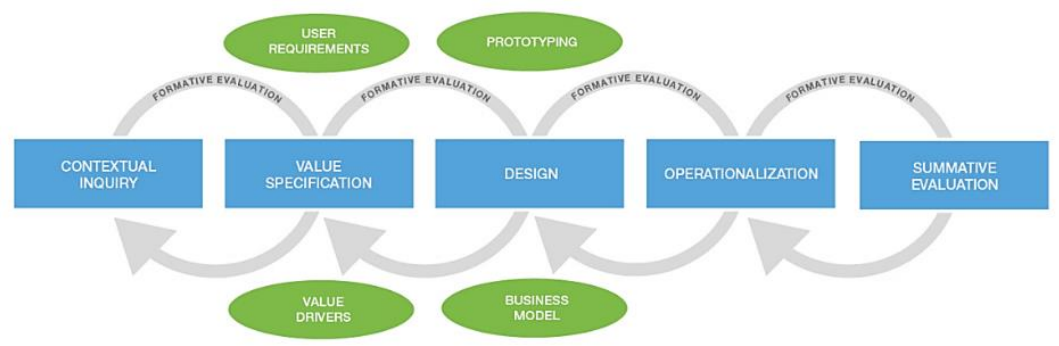

Figure 1. The CeHRes Roadmap [2].

Whereas most development processes start with the design of a technology, we consciously take a step back. The first stage of the CeHRes Roadmap is the Contextual Inquiry. In this stage, the developer seeks cooperation with known stakeholders of the technology to explore the context in which the technology must be used, and which preconditions it should meet [2]. Based on the outcomes of this inquiry, and again in cooperation with known stakeholders, it is then studied whether there are any potentially relevant stakeholders missing, and what values and needs they have. This is all done during the stage of Value Specification [2]. Then, in the Design stage, attention is given to the question of how solutions to the found problems and preconditions can be incorporated within the 
technology. A prototype of the technology is developed and evaluated by intended endusers [2].

As mentioned before, within this paper, the development process of a single app will be described, i.e., the Prevalence App. Aim of this app is to support Elderly Care Physicians in nursing homes during the registration of their clients during prevalence measurements of Healthcare Associated Infections (HAls). HAls form an increasingly pressing threat to patient safety [7]. To be able to adequately protect patients against this threat, one of the first steps is to gather knowledge on its occurrence [7]. For hospitals, already a vast amount of surveillance data is available [7-13]. For other healthcare institutions (such as nursing homes), prevalence studies have more recently begun to take place. The results of the first prevalence study of HAls in Dutch nursing homes were published in 2011 [14].

To enable prevalence research in nursing homes, data must be collected on all clients that are present in the nursing homes at one point in time. The success of such data collection is entirely dependent on the willingness and capability of the elderly care physicians to register their clients in a correct and timely manner. Preferably, the registered data should be collected in a standardized way to fit other (e.g., nation-wide) surveillance programs [7][8]. The prior registration system consisted of many (often irrelevant) long and very complicated questions, which are prone to interpretation errors. Physicians sometimes doubted the exact meaning of the questions, which could harm the reliability of the data. Registration via this system also took its users quite some time.

The eventual aim of the new registration system is to use user-centered and persuasive design to optimally support nursing home physicians during the registration of their clients in HAl prevalence measurements. However, for the current paper, that is not the main purpose or focus. Here, we describe the process towards achieving such aim. To reach the end goal, our approach is applied. In this paper, we describe how UCD and PSD can together contribute to the development of a successful registration system for HAls in nursing homes.

\section{Methods}

\subsection{Contextual Inquiry - An Expert Discussion}

This project started with a request from iPrevent [15] to aid in the development of a new 'mobile' registration system. iPrevent is a regional infection control network within which healthcare institutions, elderly care physicians, medical microbiologists and Infection Control Professionals (ICPs) work together to structurally offer high quality care in the field of infection prevention [15].

Instead of immediately initiating the development and design of a technology, the CeHRes approach required us to take a step back, to perform a Contextual Inquiry [2]. Therefore, to gain insight into what iPrevent specifically wants or needs from this project, and into the prerequisites for the registration system, an expert discussion was held.

\subsubsection{Participants}

Participants in the expert discussion were invited to the meeting via e-mail. Two of them are project leaders (a medical microbiologist and an ICP) of the iPrevent network; they are also the initiators of the development of a Prevalence App. One of the project leaders is also the data analyst, who is responsible for collecting, analyzing and reporting the 
registration data (both for publication purposes and as feedback towards the participating nursing homes). Also, two behavioral researchers, specializing in UCD for persuasive technology, participated in the expert discussion.

\subsubsection{Procedure}

The expert discussion included relatively few people. Therefore, it was decided to keep the setting and discussion informal. The expert discussion thus took the shape of a conversation, during which the behavioral scientists did ask additional questions to acquire all the information that was necessary for the development of a new registration system, e.g., who have an interest in the performance or results of the prevalence measurements, and why.

\subsubsection{Data-analysis}

Notes were made by one of the behavioral researchers, during the entire discussion. These were used to form a 'working document' that would be used as a communication tool (between behavioral researcher and project leaders) during the entire development process. In the document, the outcomes of the discussion were complemented with literature about the registered HAls and national surveillance programs. This was done to make sure the registration system would fit the context as described by the experts.

\subsection{Value Specification - A Questionnaire}

Then, following the CeHRes Roadmap, the Value Specification stage was initiated, to gain insight in what values end-users had (concerning a registration system) and whether the project aims match these values [2]. For this purpose an online questionnaire was developed, based on the results of the Contextual Inquiry.

\subsubsection{Participants}

Elderly care physicians were invited to participate, by one of the project leaders, via email. They were informed about the Prevalence App that was being developed, and that their input was needed to make sure that it would fit their needs and work processes. A total of 24 elderly care physicians, who worked at different nursing homes within the iPrevent network, were willing to participate in the study. Their ages ranged from 30 to 61 (mean age 47 years). Most participants were female (19 female vs. 5 male).

\subsubsection{Procedure}

The invitation e-mail included a direct link to the questionnaire. The questionnaire consisted of some basic questions about the demographics of the respondent, his / her technology use and how the registration is currently being performed. Then, questions were asked about how relevant the physician feels the prevalence measures are, and how, when and via what kind of device the physician prefers to register clients. Additionally, questions were included about what information or feedback the physician expects to get from the registration system and what would motivate them to adopt a new system.

\subsubsection{Data-analysis}

Questionnaire results are analyzed to obtain descriptive statistics. Further statistical analyses were deemed redundant, given the developmental purpose of the study. Answers that are given to open-ended questions were summarized if they overlapped and then their frequencies were analyzed as an indication of how many respondents share a specific opinion. 


\subsection{Design - In-Depth Interviews and Scenario- Based Tests}

Next step in combining user-centered and persuasive design is to start the Design stage of the CeHRes Roadmap [2]. Thus, to validate the questionnaire data and to optimize the user-friendliness and persuasiveness of the prototype, scenario-based user-tests and indepth interviews with end-users are performed.

\subsubsection{Participants}

Participants were invited to the study by one of the project leaders. If they agreed, they were contacted by the researcher to schedule a meeting at a location that was convenient for the participant. Four female elderly care physicians who worked in different nursing homes within the iPrevent network participated. Their ages varied from 33 to 59 (mean age 45 years). One of the nursing homes they worked at, already used Electronic Client Files (ECFs), the others expected they would start to do so in the near future.

\subsubsection{Procedure}

First of all, a prototype of the Prevalence app was developed, in close cooperation with an $I C P$, using Balsamiq software [16]. It was based on the outcomes of the expert discussion, about the context of requirements of the registration. The prototype incorporated elements of the PSD model [4]. Not all elements of the PSD model were deemed relevant, only elements that are relevant in this specific context and for this specific app have been applied.

Then, two scenarios were developed to be used in the user-tests. They were developed in close cooperation with an ICP and made use of literature on the HAI definitions. The scenarios addressed critical issues for registration e.g., a client with multiple infections or a lab test that has been performed without the results being known yet.

The physician was instructed to talk out loud during the entire user-test, not only mentioning what she thought, but also what she saw or sought, did or wanted to do during the registration of the fictional client. The entire conversation was audio recorded, with permission of the participant.

\subsubsection{Data-analysis}

The conversations, including both interview and user tests, took about 45-60 minutes each. Audio recordings of the conversations were transcribed verbatim and analyzed using a code book, which aided in the structuring of data. Some examples of the codes are given in Table 1. All codes were combined and the frequencies with which they were mentioned were analyzed.

Table 1. Examples of codes used for analysis of user-tests

\begin{tabular}{|c|c|c|}
\hline Category & Code & Description \\
\hline Contextual Inquiry & $\mathrm{Cl}$ & $\begin{array}{l}\text { Subject describes a problem that is experienced in the } \\
\text { current work process }\end{array}$ \\
\hline Value Specification & V1 & Subject thinks working with the new system might be faster \\
\hline Design & D1 & Subject thinks the order of the items in the mock up is wrong \\
\hline Operationalization & 01 & $\begin{array}{l}\text { Subjects talks about a possible barrier for using the new } \\
\text { system }\end{array}$ \\
\hline
\end{tabular}




\section{Results}

Aim of the current paper is to describe how UCD and the PSD model can simultaneously and complementarily be applied, to develop successful eHealth technology. For that purpose, the development process of the Prevalence app is used. In this section, we will describe the results that were rendered during the different stages of the CeHRes Roadmap.

\subsection{Contextual Inquiry - Description of the current situation}

First of all, the Contextual Inquiry generated more in-depth insight into the current situation (the situation prior to the development of a new registration system).

iPrevent has, over the last years, worked together with the approximately 30 nursing homes within their network, to perform annual prevalence measurements for HAls in nursing homes. This implies that the elderly care physicians are required to once a year register all relevant data about the residents that live in their nursing homes. This not only entails information about the presence of HAls, but also about the presence of risk factors for the occurrence or spread of infections. Risk factors are e.g., the use of antibiotics or catheters, or staying in a room with multiple other clients. Inherent to the fact that it is a prevalence measurement, all clients must be registered within a short timeframe around a reference date.

The content of the registered data is largely determined by the given definitions of HAls. These definitions were developed by the regional network in cooperation with the national surveillance system (PREZIES) [17]. In the hospital setting, surveillance is performed by trained infection control nurses. The use of the definitions in the nursing home setting, where registration is performed by physicians with little or no experience with surveillance, registration is far more complicated. The prior registration system directly used the definitions of $\mathrm{HAl}$ as questions. It did not, however, offer additional explanation or clarification about their meaning, or any other kind of support with answering the questions. Physicians had indicated that they sometimes debated with their colleagues about how to interpret and answer a certain question (see Quote 1). This, of course, caused some issues with standardization and analysis of data.

(Quote 1 - Originally in Dutch) "[...] The way the questions are asked. They
are not always clear, even though that is important. Now, I sometimes have
to go to one of my colleagues to ask them, like: hey, how do you answer this?
And it is important that everyone registers in the same way."

The prior registration system consisted of an online questionnaire, developed by experts in the field of Infection Prevention and Control. The questionnaire consisted of a long list of complicated questions (see for example the screenshot of the prior registration system in Fig. 2). Many of them are irrelevant for most residents. For example, if a client does not use an antibiotic, the question about what an antibiotic is used for, is rather redundant. Also, most questions were presented on a single page. Physicians thus had to scroll down for quite some time. They had to read all questions, including irrelevant ones, to check whether they applied to the client. 
2. If there currently is an infection, was it already present at the time of admission to the nursing home?

Yes, infection currently present, was present during admission and admission $<2$ weeks ago (=end of registration)

Yes, infection currently present, was present during admission but admission $>2$ weeks ago (=continue registration)

Not applicable, currently no infection present or infection is currently present, but was not present during admission

\section{Prev. Next}

Figure 2. Screenshot of the prior registration system

Furthermore, an increasing amount of nursing homes $(>30)$ participate in the prevalence measurements that iPrevent performs. Thus, increasingly large datasets are collected. Data processing, data analysis and presentation of feedback of the results to the nursing homes were all performed by a single data-analyst. This will soon no longer be feasible. Therefore, project leaders (and the data-analyst) would like the system to perform these tasks automatically. Finally, project leaders requested that the new system would be 'mobile' (to be used on a smartphone) to enable bedside registration of clients by elderly care physicians.

\subsection{Value Specification - Users' Needs and Values}

For the second stage of the CeHRes Roadmap, the questionnaire that was used resulted in insight in the users' values and needs. First of all, most (83,3\% out of 24$)$ participants indicated that they did consider prevalence measurements of HAls in the nursing home setting to be relevant. They found it important since it contributes to gaining insight into the current status of HAls in nursing homes, and enables organizational policies to be adapted to the findings. One of the participants also considered it to be of great importance for the quality of care (see Quote 2).

(Quote 2 - Originally in Dutch) "Thus far, too little research has been performed, among the nursing home population, to be able to act in a meaningful and evidence based way."

When asked what kind of device they would like to use for the registration, most subjects indicated they preferred a PC $(50,0 \%)$ or laptop $(20,8 \%)$. The other subjects preferred to use a Smartphone $(12,5 \%)$, tablet $(8,3 \%)$, or paper $(8,3 \%)$.

Most important reasons for users to be willing to use the new registration system were: (1) if they can interrupt registration without losing data; (2) if the new system is more user friendly; (3) if it can be opened simultaneously with ECFs; (4) if clear insight is given in the results; and (5) if registration can be performed faster. 
One of the prerequisites that were found during the expert discussion said that it would be desirable if registration could be performed at the residents' bedside. This would enable the physicians to directly see how the resident is doing. However, the questionnaire showed that none of the physicians considered this to be desirable. Most of them $(54,2 \%)$ did indicate that it would be of added value for them to be more flexible in the location in which they register their clients, but didn't want to do so at bedside. Also, 41,7\% said that it would not be of added value at all since they just liked to register their residents in their offices. One physician (4,2\%) wanted to register her clients in the department's office, with the client files at hand.

The in-depth interviews gave even more insight into the situation. The clients that are to be registered with the prevalence app are mainly elderly people, who have health issues. Unfortunately, these elderly clients are quite often also somewhat lonely. So, upon doing their rounds or checking up on their clients, the elderly care physicians had experienced that clients were in need of attention and wanted to interact with them. The physicians felt it would be poor bedside manners to be standing next to a client, while being entirely focused on the registration. At the same time, if they were to pay more attention to the client, they feared that the registration would take up to much time, or would be prone to errors due to a lack of concentration. They are therefore opposed to bedside registration of clients.

At the same time, physicians did indicate that being 'mobile' during registration would have advantages. They explained that their nursing homes were (going to be) using ECFs. This is software that contains highly personal and private information about the residents. Therefore, many safety measures have been taken to protect this information. Because of one of these safety measures it is impossible to simultaneously open the ECF and the World Wide Web. In practice, this meant that elderly care physicians had to open the ECF, and write down all the information about all of their clients that they needed to register in the prevalence measurements. Then they had to close the ECF, open the registration system and enter the information they had written down. Not to mention that if they had forgotten any information, the entire procedure had to be repeated. Therefore, some subjects did want registration to be possible on a mobile device, but for reasons that differed from what was expected, i.e., so they could simultaneously open the ECF on their pc and the registration system on the other device. 


\subsection{Design - Developing and evaluating a prototype}

The results that were generated in the Design stage are twofold: (1) we can now describe how we applied certain elements of the PSD model to the prototype of the prevalence; and (2) we can make a brief analysis of the user-friendliness and perceived persuasiveness of the prototype of the app.

\subsubsection{The use of the PSD model in the Prototype}

Here, a description is given of how elements of the PSD model were incorporated in the prototype. This is intended to benefit other eHealth technology developers, who might use it as an example for how the theoretical constructs of the PSD model can be applied in practice.

\section{Primary Task Support}

One of the main concerns with working with the prior registration system was that working with it required the physician to read through many irrelevant questions for every client. One of the most important elements of PSD that were to be used in the new system was therefore tunneling [4]. Tunneling is defined by Oinas-Kukkonen and Harjumaa as 'Using the system to guide users through a process or experience' [4]. The prototype system was designed to guide the user through the process of registration: questions to be answered are dependent on the answers given to prior questions. Thus, the entire system is one big decision tree, to make sure every client is registered via the shortest (fastest) possible route.

Another concern with the prior registration system was the complexity of the used questions: a single question could actually consist of multiple individual questions (see for example Quote 3). To reduce the complexity, and thus the risk of interpretation errors, reduction was used. The aim of reduction is 'to reduce complex behavior into simple tasks, to help users perform the target behavior' [4]. This was done by translating complex and long questions into multiple shorter and easier questions with a routing structure between them. For example, the originally used question for Gastro-Enteritis, was rather lengthy and complicated (see Quote 3).

Does the resident have Gastro-Enteritis? The diagnosis Gastro-Enteritis is given

if one of the following symptoms occurs in the client:

- Three times or more diarrhea (different from normal for this client, frequency is not applicable when using incontinence materials)

- Diarrhea and two of the following symptoms: fever, vomiting, nausea, stomach ache, stomach cramps, blood or mucus in feces.

- Vomiting three times within 24 hours, without any additional symptoms (if vomiting is not associated with medicine use)

- Vomiting and two of the following symptoms: fever, nausea, stomach ache, stomach cramps, blood or mucus in feces.

Quote 3. Question about Gastro-Enteritis in the prior registration system (Originally in Dutch)

The different elements of this one question were split up into multiple questions. These were one-by-one presented to the users. A build-in logic system directed the users to the appropriate follow-up questions on different screens. Some of these screens are shown in Fig. 3. 


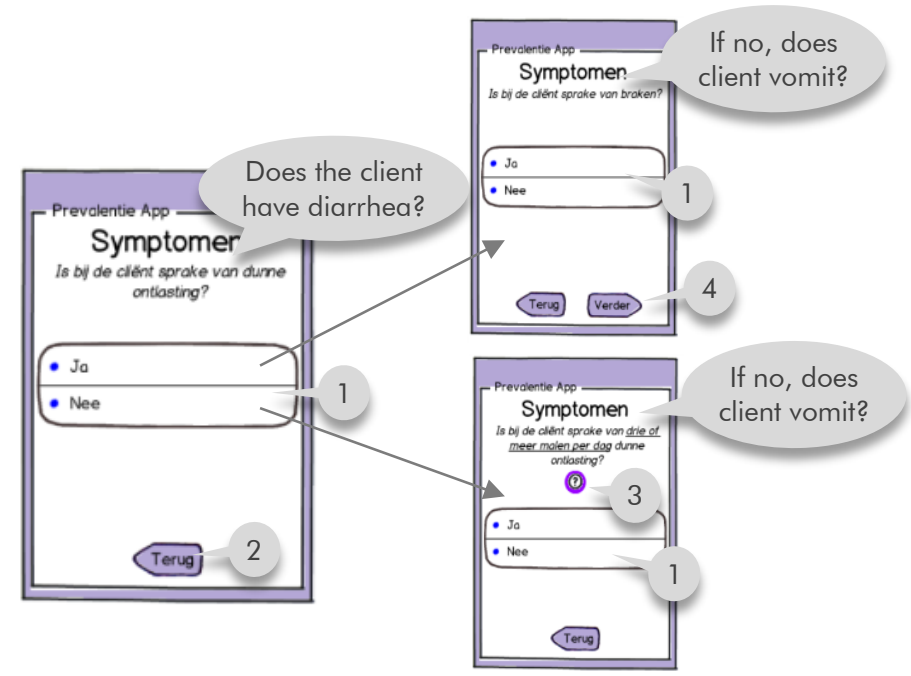

\section{(1) Answer options: Yes / No; (2) Button to return to previous screen; (3) Help-button for additional information; (4) Button to continue to next screen.}

Figure 3. Part of the screens of the prototype used for Gastro-Enteritis; Example of Reduction As mentioned before, it was of great importance that registration would be faster and could be paused without losing data. To enable this, elements of tailoring are applied to the prototype. Tailoring, according to Oinas-Kukkonen and Harjumaa, stands for 'the adaptation of the offered infor mation to the potential needs, interests, personality, usage context, or other factors relevant to a user group' [4]. In this case, the system tailored the information to the usage context factors. Every nursing home was given a unique log-in code and password. A physician had to log-in once, and was then able to continue registering their clients one after another. Moreover, when starting the system, physicians are given two options: to register a new client or to edit data of an existing client (see Fig. 4). For the latter, an overview was generated of all clients that had previously been registered by that specific nursing home. Clients of other nursing homes are not shown. By selecting a client, their data are shown and can be adjusted.

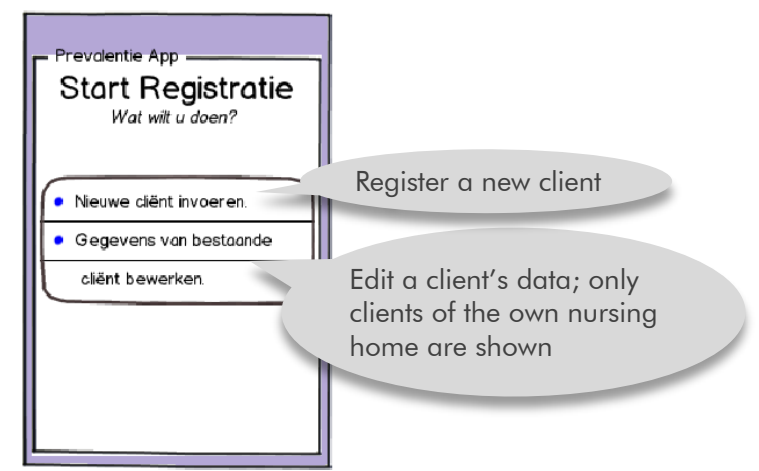

Figure 4. Example of a screen that leads to Tailored information 


\section{Dialogue Support}

Dialogue support stands for the feedback that a system gives to its users, to help them keep moving towards their goal (completing the registration) [4]. In this case, the prototype registration system requires physicians to indicate which pathogens caused an infection. However, during the user tests it became clear that within nursing homes, little funding is available to perform the laboratory tests to acquire this knowledge. Furthermore, if a laboratory test was performed at the moment of registration, its results were not always known yet. Therefore, in the final registration system, questions are added to ask whether a laboratory test was performed. If yes, the system inquires whether its results are already known. If the latter question was answered with 'no' a pop-up screen appeared. This screen reminder was shown, which means that the system reminds its users of their target behavior [4]. In this case, the reminder made the physician aware that lab results should be added later.

Also, suggestion [4] is added to the final registration system. According to Oinas-Kukkonen and Harjumaa 'systems offering fitting suggestions will have greater persuasive powers' [4]. Within the prevalence app, this is done by letting the system offer fitting suggestions for reaching the goal of registering clients. A suggestion might concern a suggested behavior, but in the case of the prevalence app, it consisted of suggestions in the sense of finishing the words that the user started to type. For example, physicians have to register all antibiotics that are used by their clients. However, the variety of antibiotics that exist is enormous and their names are complex. Initially, the intention was to let physicians scroll through an alphabetical list of generic names of all available antibiotics (see Fig. 5).

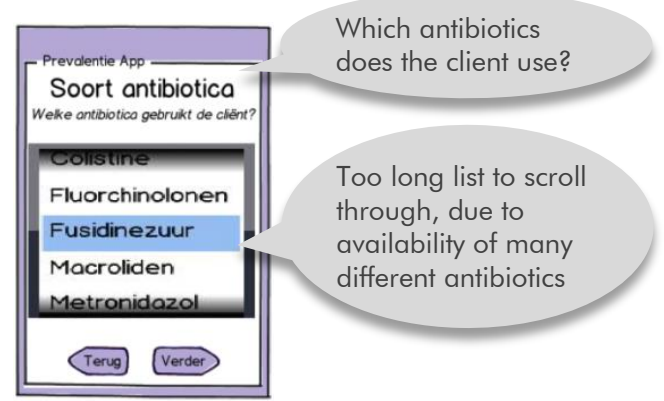

Figure 5. Example of the screen that subscribes the need for Suggestion

However, due to the large number of antibiotics that are available, this was still too time consuming. Therefore, in the final registration system, a search system in which the physician enters the first three letters of the antibiotic is added. The system then automatically generates only antibiotics that start with these letters. The same search system was then used for selecting the pathogens that have caused the infection.

\section{System Credibility Support}

Finally, in healthcare in general and for the registration of HAls in nursing homes specifically, it is of great importance that the system is credible according to its users. Therefore, a website was created about the services provided by the registration system [18]. The Infection Manager website was developed within the (INTERREG IVa) EurSafety Health-net project [19]. This is a large and successful European cross-border project, involving many hospitals, microbiology laboratories and other healthcare institutions. It aims to increase patient safety by preventing (the spread of) HAls. The part of the Infection 
Manager website about the registration system consists of e.g., background information about the project and the parties that were involved in the development process. This was intended to give more clarity about the trustworthiness and expertise of the project and its project members [4].

Lastly, in the final registration system, it was decided to add the EurSafety Health-net logo, to give the system more surface credibility [4]. This entails the initial assessment that users make about the credibility of the system, based on first hand inspection [4].

\subsubsection{Analysis of the User-Friendliness of the Prototype}

User-testing the developed prototype is aimed at evaluating whether the elements of PSD have indeed contributed to the development of a user-friendly and persuasive eHealth technology. Based on the performed user-centered scenario-tests, we found that issues with the prior registration system had indeed been resolved, or had at least been improved. For example, to implement the concept of tailoring, elderly care physicians now had to log-in to be able to register their clients. Besides allowing us to tailor the presented information, it also had other (unintended) benefits: some questions had become redundant, which could possibly speed-up the registration process (see Quote 4).

(Quote 4 - Originally in Dutch) "Cause now, I have to indicate every time: Nursing home X, Department so-and-so. But we fill in the registration for one entire department. So it saves time if we would only have to fill it in once."

According to the physicians, an additional advantage of the log-in system is that registration can be paused without losing the data (see Quote 5).

(Quote 5 - Originally in Dutch) "Well, the advantage is that one can keep adding (new) clients. You could for example continue at a later moment. Such as when you only have time to complete the registration of 10 clients, then register 10 now, and just continue two hours later."

Still, major and minor adjustments had to be made in the mock ups. These concerned the clarity of wording, sequence of questions, completeness, user-friendliness, design and location of the buttons. For example, initially, there were two screens in our prototype for 'Aids', which asks whether the client uses any aids such as a catheter or tracheotomy; and 'Incontinence', which asks whether the client is incontinent. During the user-tests, the subjects had several comments about these screens. First of all, whereas we interpreted the term 'aid' as being a catheter of some kind, the subjects indicated that the term 'aid' to them meant 'walker' (see Quote 6). So, they suggested using a different term.

(Quote 6 - Originally in Dutch) "Yes, we use the word 'Aid' for something completely different. We use this word for walkers. So I would try to come up with a different word here."

Furthermore, they found the screen about incontinence unclear. In one of the scenarios, a client was described who had a catheter. The participants indicated that, although incontinence is a possible reason for clients to get a catheter, they did not consider this client as being incontinent anymore (see Quote 7). Therefore, they said the option of having a catheter or stoma should be added to this screen. 
(Quote 7 - Originally in Dutch) "You see, this client is not incontinent, but has a urethra catheter... So this is strange. You should add catheter here I guess. Because with a catheter you are not really incontinent anymore."

As a result, the two screens where replaced by a new screen. This screen asks whether a client is incontinent. However, an additional answer option has been added, to indicate that a client has a catheter or stoma.

After the fourth user-test, no major issues where found anymore. Therefore, meetings with Information and Communication Technology (ICT) developers were held to further discuss how to incorporate the requirements into the registration system and to finally develop it.

\section{Discussion}

As far as the authors know, this study was the first to demonstrate how User-Centered Design (UCD) and the Persuasive Systems Design (PSD) model can be combined, and can even complement each other, in the development of easy to use eHealth technology. It was intended to provide insight into the steps that might be taken for applying elements of the PSD model during the developmental stages of an eHealth technology. It did so, by describing the development process of a single app: the Prevalence App.

First of all, the use of UCD has proven its benefits: the constant and structural cooperation with end-users during the development process, gave us the opportunity to make it an iterative and reflexive process. This means that it was possible to evaluate the eHealth technology with end-users, in every stage of its development, and to (at any time) adjust the direction that it was going in. This aids in the dynamic development of an eHealth technology that fits its users' needs and context, and could potentially prevent high costs of re-design if major necessary adjustments are only found after final release of the technology.

Second, this paper has shown that the PSD model is indeed useful and applicable for the design of any kind of eHealth technology. However, and more importantly, we believe that it is the combination of the two theoretical frameworks above, within the CeHRes Roadmap, which has proven to be of the greatest added value. We feel that by 'simply' applying the PSD model to an eHealth technology, one would not be using it to its full potential. Merely incorporating elements of the PSD model in eHealth technology, does not necessarily mean that the end-users of the technology also have a need for these elements. If there is no such need, developers are at risk of using sledgehammers to crack nuts, to be greatly overreaching themselves. This paper has demonstrated that the success of a technology is not dependent on the quantity of persuasive elements that are used. Rather, the key to success is to use elements of the PSD model in a focused and usercentered manner. This is why the combination with UCD is so beneficial. Using UCD, for instance in the form of scenario-based user-tests, developers can expose which elements of the PSD model should be used, to cope with any kind of usability issues and to create an optimal fit with the end-users.

In their paper, Oinas Kukkonen and Harjumaa present the PSD model as being part of development process [4]. They indicate that before the actual design of the technology, the first step in that development should be to understand 'fundamental issues behind the persuasive systems' [4]. Then, 'the context for persuasive systems needs to be analyzed, 
recognizing the intent, event, and strategies for the use of a persuasive system' [4]. The integration of the PSD model within the Contextual Inquiry, Value Specification and Design stages of the CeHRes Roadmap offers a structure for the application of these steps in the development of a successful eHealth technology. It offers a practical framework by which elements of the PSD model can be used in practice.

We believe that the holistic approach using the CeHRes Roadmap has provided the opportunity to further ground the PSD model. It has proven to be a very suitable tool to integrate UCD and the PSD model in a way that developers not only profit of their individual strengths, but were they also complement and further strengthen each other. Via UCD, for which we used e.g., scenario-based tests, we found essential issues during the use of the prototype. Via the PSD model, we were able to address these issues, and to eliminate or at least decrease their presence.

Thanks to the CeHRes Roadmap, we developed a registration system to optimally support elderly care physicians in the correct and timely registration of their clients, taking into account the national prevalence studies $[7,8]$ with which collected data should be compatible. Certainly, we still want to evaluate the speed, user-friendliness, fit with work processes, ease of use, clarity and persuasiveness of the final registration system. Thus, a summative evaluation is currently being planned. This evaluation will combine both qualitative and quantitative methods and will e.g., focus on user friendliness, speed of registration (both were found important by the end-users) and amount of errors that are made (important for the quality of the data). But for now, it can be said that the new system has already been used in two rounds of prevalence measurements, successfully registering over 3000 nursing home residents. It is web-based, so it can be used on any device capable of connecting to the World Wide Web. An example of a screen of the eventual registration system is given in Fig. 6.

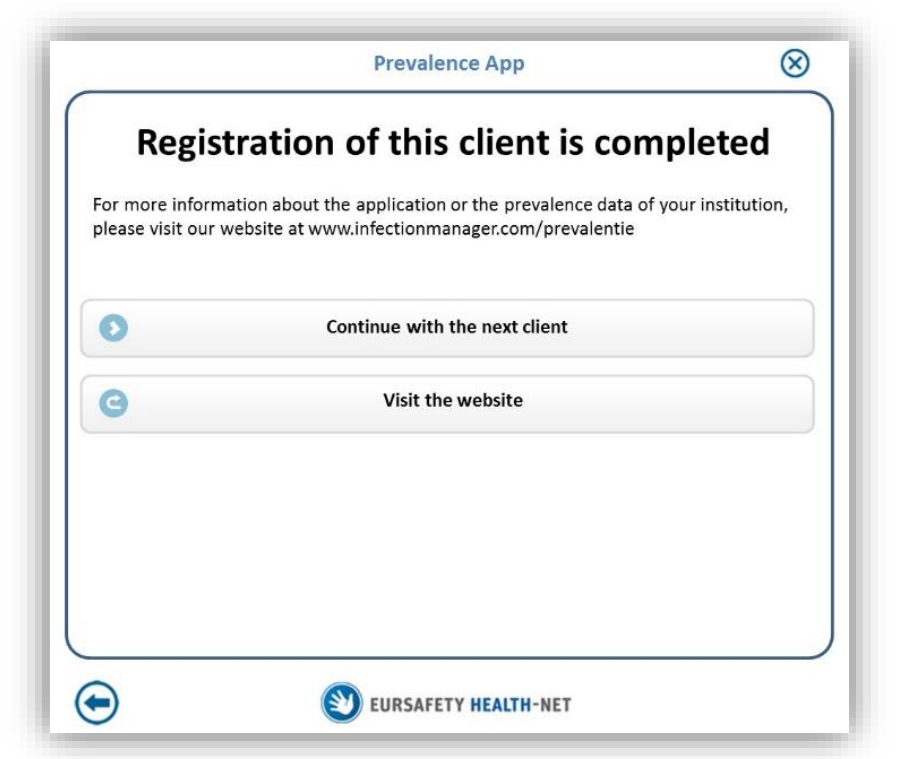

Figure 6. Example of the look and feel of the eventual registration system (Originally in Dutch) 


\subsection{Limitations}

A limitation of this study is that it suffered from very strict and ambitious deadlines. This limited us in the amount of effort that we could put into the implementation stage of the development. Although this is thought to be a very relevant stage according to the CeHRes Roadmap, in this project its influence might be limited. The elderly care physicians, who are the end-users of the registration system in this study, are obligated to use it if their nursing home participates in the prevalence measurements.

Another possible limitation might be that our study had a relatively low number of participants. However, aim of this study was solely the serve as an example of a development process of an eHealth technology, not to perform an evaluation of its effects.

Also, the prevalence measurements have to be suited to be used in national surveillance (by PREZIES). Therefore, the new registration system has to meet certain requirements (e.g., data that must be collected, or in what form). Thus, in this case, and in most cases where a new eHealth technology is being developed, legislation was part of our Contextual Inquiry and also may have had an influence on the design of the technology.

Finally, the given setting for this project (nursing homes) presented us with its very own challenges. The opportunities for using technology were limited, because of the technological infrastructure of Dutch nursing homes (wherein often outdated PCs are used), and the degree to which people are used to working with technology (e.g., only $47,1 \%$ of the physicians used a Smartphone). However, this gave us an interesting opportunity to put ourselves and the possibilities of the CeHRes Roadmap to the test, to see how it and how we would cope with such limitations.

\section{Conclusion}

This article goes beyond the mere development of eHealth technologies. It has subscribed our strong believe that combining UCD and the PSD model is of paramount importance for the creation of successful and persuasive eHealth technologies, because (1) UCD gives insight in the needs and wishes of the end-users, that have to be met by the eHealth technology; (2) the PSD model offers opportunities to deal with the issues and needs that are found using UCD; and (3) although they might have used their own words to express themselves, end-users appeared to have very clear ideas about the their needs regarding Persuasive Systems Design. Also, our approach, using the CeHRes Roadmap has allowed for the development to be an iterative process, which may prevent costly redesign to be necessary.

\section{Acknowledgments}

This project was funded by the Interreg IV "EurSafety Health-net" project. We also thank the elderly care physicians who participated in this study. 


\section{References}

1. De Jong, N., Eikelenboom-Boskamp, A., Voss, A., et al., User-centered and persuasive design of a web-based registration and monitoring system for healthcare-associated infections in nursing homes, In: Proceedings of the $6^{\text {th }}$ International Conference on eHealth, Telemedicine, and Social Medicine (eTELEMED 2014) IARIA, 2014, p. 152157.

2. Niiland, N., Grounding eHealth; towards a holistic framework for sustainable eHealth technologies, University of Twente: Enschede.

3. Van Gemert-Piinen, J.E.W.C., Karreman, J., Vonderhorst, S., et al., Participatory development via user-involvement - a case study about the development of a web-based patient-communication system about Methicillin-resistant Staphylococcus aureus, Electronic Journal of Health Informatics, 2011 , 6(4), p. e28.

4. Oinas-Kukkonen, H. and Harjumaa, M., Persuasive systems design: Key issues, process model, and system features. Communications of the Association for Information Systems, 2009, 24(1), p. 28.

5. Oinas-Kukkonen, H., Behavior Change Support Systems: A Research Model and Agenda, In: $5^{\text {th }}$ International Conference on Persuasive Technology, 2010, Springer Berlin Heidelberg, p. 4-14.

6. Oinas-Kukkonen, H., and Harjumaa, M., Towards deeper understanding of persuasion in software and information systems, In: $1^{\text {st }}$ International Conference on Advances in Computer-Human Interaction (ACHI 2008), 2008, IEEE, p. 200-205.

7. European Centers for Disease Control and Prevention, Point prevalence survey of healthcare-associated infections and antimicrobial use in European acute care hospitals, 2013, Stockholm, Sweden.

8. PREZIES. Referentiecijfers maart 2007 t/m maart 2012: Prevalentieonderzoek, 2012 cited 2014 6-11, Available from:

http://www.rivm.nl/Onderwerpen/P/PREZIES/Prevalentieonderzoek_Ziekenhuizen/Refere ntieciifers_Prevalentieonderzoek_ziekenhuizen.

9. Mayon-White, R.T., Ducel, G., Kereselidze, T., et al., An international survey of the prevalence of hospital-acquired infection, Journal of Hospital Infection, 1988, 11 (Suppl A), p. 43-48.

10. Klevens, R.M., Edwards, J.R., Richards, C.L., et al., Estimating health care-associated infections and deaths in US hospitals, Public health reports, 2007, 122(2), p. 160-166.

11. Smyth, E.T.M., Mcllvenny, G., Enstone, J.E., et al., Four country healthcare associated infection prevalence survey 2006: overview of the results, Journal of Hospital Infection, 2008, 69(3), p. 230-248.

12. Gravel, D., Taylor, G., Ofner, M., et al., Point prevalence survey for healthcareassociated infections within Canadian adult acute-care hospitals, Journal of Hospital Infection, 2007, 66(3), p. 243-248.

13. Lyytikäinen, O., Kanerva, M., Agthe, N., et al., Healthcare-associated infections in Finnish acute care hospitals: a national prevalence survey, 2005, Journal of Hospital Infection, 2008, 69(3), p. 288-294.

14. Eikelenboom-Boskamp, A., Cox-Claessens, J.H., Boom-Poels, P.G., et al., Three-year prevalence of healthcare-associated infections in Dutch nursing homes, Journal of Hospital Infection, 2011, 78(1), p. 59-62.

15. iPrevent, www.i-prevent.net, cited 2014 6-11.

16. Balsamiq, Available from: https://balsamiq.com/

17. iPrevent, Definities van verpleeghuisinfecties, cited 2014 6-11, Available from: www.infectionmanager.com/definities

18. Infection Manager, cited 2014 6-11, Available from: www.infectionmanager.com/prevalentie 
19. Wentzel, M.J., Karreman, J., and Van Gemert-Piinen, J.E.W.C., Towards an internetbased infectious disease management platform to increase patient safety, In:

Proceedings of the $3^{\text {rd }}$ International Conference on eHealth, Telemedicine, and Social Medicine (eTELEMED 2011) IARIA, 2011, p. 47-50. 



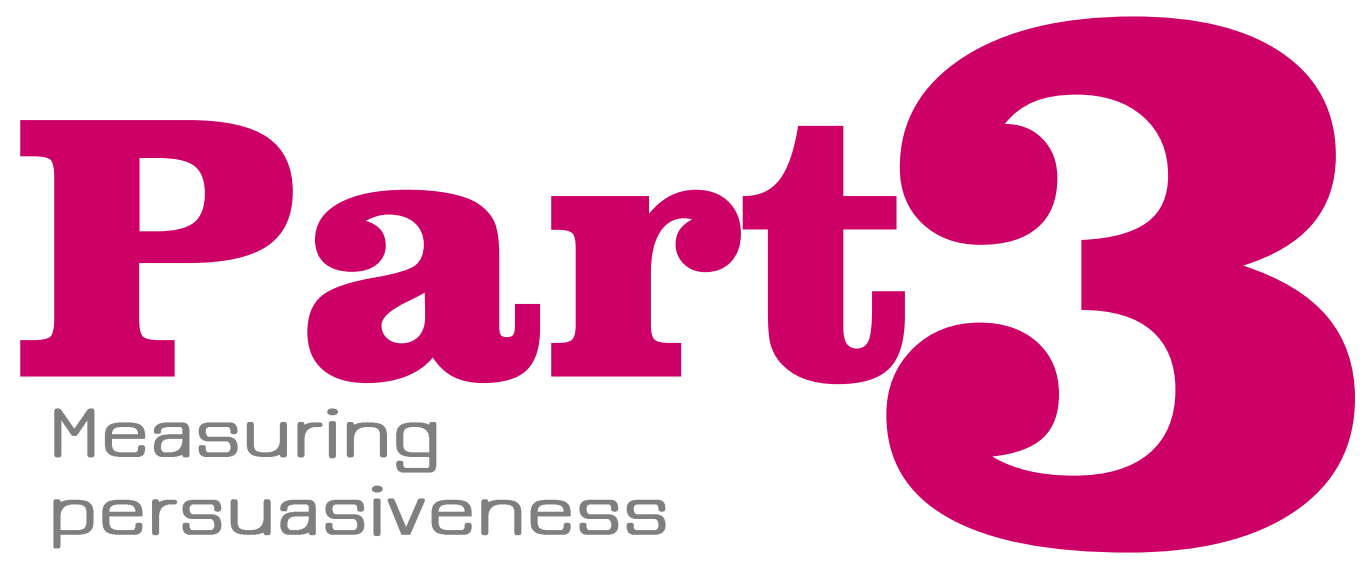





\section{Combining user-tests, log-data and expert-assessments to evaluate constructs of the Perceived Persuasiveness Questionnaire}

N. de Jong, J. Wentzel, S. Kelders, H. Oinas-Kukkonen, J. Van Gemert-Piinen Proceedings of the Second International workshop on Behavior Change Support Systems (May 22)

Persuasive 2014, Padua, Italy 


\section{Abstract}

To develop effective behavior change support systems, persuasive technology can be used. The persuasive systems design model offers a framework to identify and operationalize such elements. In this pilot study, we evaluate the questionnaire developed to measure perceived persuasiveness of information technology. We analyzed verbatim user-test transcripts, and performed expert-assessments of the Nurse Antibiotic Information App (NAIA). These data were compared to questionnaire results on this app.

Expert-assessment identified task support, perceived persuasiveness, unobtrusiveness, credibility, perceived effort and perceived effectiveness (as defined in the Persuasive Systems Design model) as being present within the NAIA. These constructs also scored satisfactory in the questionnaire. User-test transcripts are in line with questionnaire results. Given the consistent results in this pilot study, our approach seems promising for evaluating the questionnaire and will be applied to other settings and websites/applications. 


\section{Introduction}

Any interactive computing system, designed to change users' attitudes and/or behavior, is called persuasive technology [1]. Oinas-Kukkonen and Harjumaa [2] state that the changing of users' attitudes and/or behavior should be achieved without using coercion or deception. For the development and design of such technology, the Persuasive Systems Design model (PSD) can be used [3]. However, using this model during development and design of a Behavior Change Support System (BCSS), does not necessarily mean that users feel more motivated for behavior change. Therefore, Lehto et al. developed the Perceived Persuasiveness Questionnaire (PPQ) to predict the perceived persuasiveness of a BCSS [4]. However, thus far, the PPQ has not been fully validated yet. It is of importance that this is done, to be able to reliably compare different eHealth technologies, or their application within different settings, with each other. In this study, the University of Twente and the University of Oulu cooperate, to evaluate the $P P Q$, with the ultimate goal to have a validated tool to measure perceived persuasiveness available. This is important, since the PPQ offers eHealth developers an opportunity to measure the perceived persuasiveness of their technology and to test the assumptions of the PSD-model. For the validation, we evaluate the results of the PPQ in different settings, aimed at civilians, patients and professionals [4-8]. We will perform expert-assessments to determine which elements of the PSD model are actually incorporated in the ICT system under investigation.

In this paper, we describe an explorative pilot study in which a questionnaire, usability tests, and expert-evaluations are combined to evaluate PPQ constructs, applied to the Nurse Antibiotic Information App (NAIA) [5]. Research questions are:

- Does the users' perceived persuasiveness relate to expert evaluations of the presence of categories for persuasive system principles?

- Does the users' perceived persuasiveness relate to verbalized user-experiences during user-tests?

\subsection{The Nurse Antibiotic Information App}

Nurses need easily accessible, centralized information support at the point of care, especially regarding medication safety $[9,10]$. As part of an antimicrobial stewardship program (promoting prudent use of antimicrobials), the web-based NAIA [11] was developed. A more detailed description of the NAIA and its participatory development process is given elsewhere [5, 12]. The NAIA includes information on the preparation and administration, but also optionally provides additional background information.

\section{Methods}

\subsection{Study Setting}

The NAIA was implemented in two lung wards of a local 1000-bed teaching hospital. A total of 62 nurses (45 FTE) worked at these wards during the pilot phase. The app was incorporated within the nurses' personal hospital start-page, which allowed for easy access [12]. At the time of the study, the app had been available for $>6$ months at the ward, offering nurses many occasions to use it and get familiar with it. 


\subsection{The Perceived Persuasiveness Questionnaire}

The Perceived Persuasiveness Questionnaire (as it was available at the time of study) was aimed at evaluating a weight loss application. The questionnaire was used as part of a larger study for summative evaluation of the NAIA, including other measures for behavior change specifically relevant for antimicrobial stewardship [5]. The PPQ was adapted, to fit the research goals of the evaluation study [5]. This means that some constructs of the PPQ were omitted (i.e., dialogue support and social support). Thus, these are also not included in the current study. Perceived task support, perceived persuasiveness, unobtrusiveness and credibility were included. Two of the credibility items were merged, since no distinguishing Dutch translations could be formulated. We only incorporated one task support item, addressing the overall aim of the behavior change (appropriate antimicrobial use), since the behavior itself (e.g., correct administration, preparation, recognition of side effects) is too diverse to address with one item. The questionnaire was translated into Dutch and back-translated into English. Negative items were conversed and construct scores were calculated as the average score of its items.

\subsection{Expert-Assessment of Persuasive Elements Within the Nurse Antibiotic Information App}

The expert-assessment was executed by two native Dutch speaking researchers, who were both familiar with the app and its purpose. They also had several years of experience with working with the PSD model. Experts independently scored the presence of PSD constructs in a demo-version of the app. Only those constructs that can (as a persuasive strategy) be built into the technology itself, as features or characteristics of the system, were scored. This means that use continuance (the users' intention to continue working with the system [8]) was omitted as this is more an outcome of persuasive strategies than a strategy in itself. Scoring was performed on a 5-point Likert scale, differences were discussed to reach consensus.

\subsection{User-Test Analysis for Reported Persuasiveness}

Analysis of the scenario-based user-tests of the NAIA is currently work-in progress, whereas here we report on preliminary results of the summative evaluation via user-tests. It should be emphasized that, in this part of the study, nurses were not specifically asked to comment on persuasiveness elements. Rather the user-tests were aimed at the more general evaluation of the user friendliness of the NAIA. Two independent researchers analyzed the verbatim transcripts of 16 of the 34 user-tests that have been performed. This is done by scanning for any remarks, made by the nurse, about constructs of the PSD model. First, the researchers checked whether they identified the same text fragments for coding (thus, text excerpts that exemplify a persuasiveness construct). Second, they checked whether the same code was applied to the fragment. If researchers disagreed, consensus was reached via discussion about the relevance and content of particular comments. The definitions of PPQ constructs and the PSD model were used to guide the discussions. Based on the discussion, the constructs primary task support and perceived effectiveness were merged, since they overlapped greatly. For example, when users indicated that they think the app supports them in their information-search tasks, this indicates primary task support (the complex task of searching for information is made easier by using the app), but also perceived effectiveness (working with the app is beneficial for nurses in quickly and easily finding relevant information). 


\section{Results}

\subsection{Use of the Nurse Antibiotic Information App}

To gain insight in actual use of the App, log-data were recorded for eight months, between pre- and post-intervention measurement. In that period, the app was visited a 1251 times. It was used an average of 5.11 (SD 3.14) times per day. Most visitors did not only log-in but explored the App further $(10.71 \%$ of the visits consisted of viewing one page only; the entry page). On average, 5.03 pages were seen per visit, and a visit lasted on average 2 minutes and 26 seconds.

\subsection{Perceived Persuasiveness}

A total of 34 nurses were invited to complete the questionnaire, of these, 30 nurses actually participated (88.24\%). The participants' mean age was 30.8 (SD 9.06), 26 of them were female. On average, they had 8.45 years ( $\min 0.5$, $\max 38$, SD 8.52) of work experience as a nurse. They used the internet for work and private, for an average of 2.54 hours (SD 1.86) per day. Table 1 shows the accumulated, average scores of the measured constructs.

Table 1. Results of expert-assessment and PPQ questionnaire.

\begin{tabular}{lcc}
\hline PSD construct & Presence & PPQ Score* \\
\hline Primary task support & 5 & 4.25 \\
\hline Dialogue support & 1 & - \\
\hline Credibility & 4 & 4.13 \\
\hline Social support & 1 & - \\
\hline Unobtrusiveness & 4 & 4.11 \\
\hline Perceived persuasiveness & 4 & 4.11 \\
\hline Perceived effort & 4 & $\#$ \\
\hline Perceived effectiveness & 5 & $\#$ \\
\hline Use continuance & - & $\#$ \\
\hline Items were scored on a 5-point Likert scale, ranging from 1 (totally disagree), 2 (disagree), 3 \\
(don't agree, don't disagree), 4 (agree), to 5 (totally agree); ${ }^{*}:$ Negative items are conversed; \\
$\quad$ \#: At the time of study, this construct was not part of the PPQ yet.
\end{tabular}

\subsection{Presence of PSD Constructs in the App}

Participating experts reached high consensus about the presence of the PSD constructs in the app. Primary task support, credibility, unobtrusiveness, perceived persuasiveness, perceived effort and perceived effectiveness were present in the app. Consensus ratings (reached after discussion) are displayed in Table 1.

\subsection{Remarks About PSD Constructs During User- Tests}

From the verbatim user test transcripts, remarks on perceived persuasiveness were identified. The results of the analysis (including exemplary quotes) are shown in Table 2. Overall, more positive than negative remarks were made. Most remarks concerned primary task support. Perceived persuasiveness, unobtrusiveness, perceived effort and use continuance were also (positively and negatively) commented on. 
Table 2. Overview of user-test analysis results

PPQ N $\left({ }^{*}\right)$ Quote

Primary task support

Pos. $\quad 32(14)$ "[...] that it clearly shows: dose, preparation and administration. That is what I want to know. That's why I use the App."

Neg. 11(7) "I don't think it always says how long administration of an antibiotic may take."

\section{Perceived persuasiveness}

Pos. $\quad 8(5) \quad$ "[...] And it's very convenient that it is so easy to search. That's much like our good old 'yellow booklet' [paper-based antibiotic information, ed.]."

Neg. 4(3) "That's difficult to read, so it is less interesting, because you'll soon feel like you don't understand and I would then just leave it to the physician."

Credibility

Pos. 1(1) "[...] Information that you find on the internet is not specifically written for our hospital. This is."

Neg. $0(0) \quad$ n.a.

Social Support

Pos. 6(6) "Or just for your own information. [...] Because you want to be as well informed as possible when you call the physician."

Neg. $0(0) \quad$ n.a.

Dialogue support

Pos. 3(2) "As soon as you type in 'am', that Amoxicilline and Augmentin are already suggested to you. I personally find that really convenient."

Neg. $0(0)$ n.a.

Unobtrusiveness

Pos. 7(4) "This is really easy to find... just type it in and there it is! For the old systems, we had to go through many steps before you find the information you need. That's much easier here."

Neg. 5(4) "[...] It might be convenient, that if you have a EPS**, you can select the drug and are automatically brought to the information and don't have to open the App separately."

Perceived effort

Pos. 6(4) "This nicely describes how to prepare the antibiotic, while that [prior information source, ed.] requires you to read through the whole story, before you find the 'preparation' heading."

Neg. 6(3) "I notice that I am using it [the app] increasingly often, but I still have to search for a little while."

Use Continuance

Pos. 7(7) "Well, as far as antibiotics are concerned, I check the app. At least I do, and I think my colleagues do too."

Neg. 3(2) "Augmentin [an antibiotic, ed.] is something we use very often, so I don't really check the app for that."

*number of unique participants making one or more remarks in this category

** EPS: Electronic Prescribing System 


\section{Discussion}

This study combined user-tests, and expert-assessment to evaluate constructs of the Perceived Persuasiveness Questionnaire. Log-data show that, over the eight months between pre- and post-intervention measurement, the Nurse Antibiotic Information App (NAIA) is structurally being used relatively frequently, repeatedly motivating nurses to lookup information. This indicates that the NAIA was incorporated in daily clinical practice, and fulfils a need for easily accessible and well-structured information about antimicrobials. This was also found in prior research [5].

Agreement between experts about the presence of different constructs of PSD was high. Primary task support, credibility, unobtrusiveness, perceived persuasiveness, perceived effort and perceived effectiveness were found in the app. The constructs perceived effort, perceived effectiveness and use continuance were added to the PPQ after the evaluation study of the NAIA. They are therefore omitted in the questionnaire, but they all are included in the user-tests and perceived effort and perceived effectiveness are included in the expertevaluation.

All four constructs, that experts rated as being present in the NAIA, and that were included in the PPQ at the time, were also positively perceived by the nurses in the questionnaire study (score $>4$ ). The user-tests analysis showed similar results: positive remarks are made concerning primary task support, perceived persuasiveness and unobtrusiveness. Credibility did not get as many remarks; it appears to play a relatively smaller role in the practical use of the app. However, even though nurses did not proactively mention the credibility of the app, when asked (with the questionnaire), the app is considered to be credible. So, the mere fact that it was not mentioned, does not necessarily mean it is absent in the app.

A remarkable finding, based on the discussions during the user-tests analysis, was that primary task support and perceived effectiveness had to be merged. Researchers were unable to structurally distinguish these constructs within the users' comments (the comments simultaneously fitted-in with both constructs). This might be due to the nature and purpose of the app (which is directly aimed at influencing the task performance of nurses), but it might also be an indication of the importance of having a validated Perceived Persuasiveness Questionnaire available, to be able to distinguish between constructs. This pilot study only included a single system, it is therefore impossible to determine which of the two (the system or the questionnaire) caused the problem mentioned above. To avoid such bias (caused by including a single system), we will include multiple apps in the validation study. The currently studied app mainly focuses on primary task support. For the validation study, it is necessary to cover the full range of constructs of the PSD model. Therefore, other apps are included, that might aim at different constructs (e.g., Facebook, Twitter, Prevalence app, Ned i Vekt and Virtual Health Check). These have different aims (e.g., social interaction, weight loss, infection control) and different target audiences (e.g., professionals, civilians). The PPQ has, in more or lesser extent, been applied to all of these apps, which allows for comparisons to be made.

With this pilot study, we have shown that the PSD model generates consistent results, when measured using different methods. However, results of this study should be interpreted with care, due to some limitations. As this was a pilot study, it had a relatively low number of participants (users and experts). Also, not all constructs of the PPQ were included in the 
questionnaire study. Finally, its results may have been influenced by other questionnaires that were simultaneously used (concerning e.g., usability and empowerment).

In future research, we will do more in-depth log-file analyses, focusing on which parts of the NAIA are mainly used and at what moments, as prior research has shown that logfiles may be used to study the effect of persuasive elements in eHealth technology [13, 14]. Additional user-tests will be analyzed, to allow for conclusions in the field of effectiveness of the NAIA. The study will, as mentioned before, additionally be applied to other apps and other settings, and will be complemented with thorough evaluation of a Dutch PPQ. These are all important steps to be taken to enable valid PSD evaluations in summative research. The current pilot study gave us a framework, based on which we will work towards validating the PPQ. We created a protocol for expert-assessment of a behavior change and its support system, we showed how this evaluation enables a PSD focus, and provided an example of validation via user-tests. 


\section{References}

1. Fogg, B.J., Persuasive Technology: Using computers to change what we think and do, 2003: Morgan Kaufmann Publishers.

2. Oinas-Kukkonen, H., and Harjumaa, M., Towards deeper understanding of persuasion in software and information systems, In: $1^{\text {st }}$ International Conference on Advances in Computer-Human Interaction (ACHI 2008), 2008, IEEE, p. 200-205..

3. Oinas-Kukkonen, $\mathrm{H}$. and Harjumaa, M., Persuasive systems design: Key issues, process model, and system features. Communications of the Association for Information Systems, 2009, 24(1), p. 28.

4. Lehto, T., Oinas-Kukkonen, H., and Drozd, F., Factors Affecting Perceived

Persuasiveness of a Behavior Change Support System. In: '33th International Conference on Information Systems, 2012, Association for Information Systems, Orlando, Florida.

5. Wentzel, J., and Van Gemert-Piinen, J.E.W.C., Antibiotic Information App for nurses. In: Proceedings of the 6th International Conference on eHealth, Telemedicine, and Social Medicine (eTELEMED 2014), IARIA, 2014, p. 174-179.

6. Beerlage-de Jong, N., Eikelenboom-Boskamp, A., Voss A., et al., Combining usercentered design with the persuasive systems design model; the development process of a web-based registration and monitoring system for healthcare-associated infections in nursing homes, International Journal on Advances in Life Science, 2014, 6(3\&4), p. 262271.

7. Lehto, T., Oinas-Kukkonen, H., Pätiälä, T., et al., Consumers' Perceptions of a Virtual Health Check: An Empirical Investigation, In: Proceedings of the 20th European Conference on Information Systems (ECIS 2012), 2012, 154.

8. Lehto, T., and Oinas-Kukkonen, H., Explaining and Predicting Perceived Effectiveness and Use Continuance Intention of a Behavior Change Support System, Behaviour and Information Technology, 2014, 34(2), p. 176-189.

9. Koch, S.H., Weir, C., Haar, M., et al., Intensive care unit nurses' information needs and recommendations for integrated displays to improve nurses' situation awareness, Journal of American Medical Informatics Association, 2012. 19(4), p. 583-590.

10. Ndosi, M. and Newell, R., Medicine information sources used by nurses at the point of care, Journal of Clinical Nursing, 2010, 19(17-18), p. 2659-2661.

11. Demo Information Application for nurses, in Dutch, cited 2014 03-10, Available from: http://abnurseapp.infectionmanager.com.

12. Wentzel, J., Van Velsen, L., Van Limburg, M., et al., Participatory eHealth development to support nurses in antimicrobial stewardship. BMC medical informatics and decision making, 2014, 14(45).

13. Van Gemert-Pijnen, J.E.W.C., Kelders, S.M., Bohlmeijer, E.T., Understanding the usage of content in a mental health intervention for depression: An analysis of log data, Journal of Medical Internet Research, 2014, 16(1), p. e27.

14. Kelders, S.M., Van Gemert-Piinen, J.E.W.C., Using log-data as a starting point to make eHealth more persuasive, In: S. Berkovsky, J. Freyne, eds, Persuasive Technology:

Springer Berlin Heidelberg, 2013, p. 99-109. 



\section{Evaluation of the Perceived Persuasiveness Questionnaire \\ N. Beerlage-de Jong, O. Kulyk, L. Kuonanoja, J. Wentzel, H. Oinas-Kukkonen, J. Van Gemert-Pijnen International Journal of Human-Computer Studies (Submitted)}




\section{Abstract}

The Perceived Persuasiveness Questionnaire (PPQ) was developed to study and explain the working mechanisms of persuasion. It measures whether aspects of the Persuasive Systems Design model (which it was based on) are perceived by the users of a technology. However, its reliability and (construct) validity have not been elaborately studied yet. We present the results of a mixed-method study that was performed to explore whether PPQ constructs are rightfully part of the questionnaire and whether PPQ items adequately represent its constructs. Participants were 65 Dutch and Finnish university students. A closed card sort task with PPQ items and constructs was performed to gain insight in the construct validity of PPQ constructs. This was combined with a subjective evaluation of RunKeeper application using the PPQ, to gain information about the internal consistency of the PPQ items.

The results show that the PPQ offers a promising and much needed tool to evaluate whether the persuasive elements of a technology are experienced by its users. At the same time, in its current form the PPQ also has some limitations: internal consistency and construct validity vary strongly and are relatively low. We strongly suggest and we will be going back to the drawing board to study the best way to cover the full scope of the PSD model and to make best use of its strengths in a "PPQ 2.0". 


\section{Introduction}

What works best: if people are aware of persuasive elements in eHealth technology, or if they are not? This is one of the questions that are inherent to the use of modern eHealth technology, as many of such systems use persuasive design to increase adherence and to influence its users' (health) behavior [1, 2]. To offer a framework for the development and evaluation of such persuasive systems, the Persuasive Systems Design (PSD) model was developed [3]. Among others, the PSD model offers practical guidelines for the design of various system features and for how these design principles can be applied [3]. To date, even though the aim of persuasive technology is to change users' attitudes and/or behavior, it often does not achieve the intended goals and the effect of specific software design features on persuasion is still to large extent unknown.

To be able to open 'the black box' of persuasion, to study and explain the working mechanisms of persuasion in more detail, validated evaluation measures are needed. Some efforts have been undertaken to develop such evaluation measures. The Persuasive Potential Questionnaire is currently being developed to evaluate persuasiveness of a technology during its development [4]. However, since it is in practice not always possible to develop a technology from scratch, it is of paramount importance to be able to evaluate persuasiveness not only during development, but also after implementation of a persuasive technology. For this purpose, the Perceived Persuasiveness Questionnaire (PPQ) was developed, based on the PSD model [5].

The PPQ can be used as a subjective evaluation measure to try and answer the question that was stated at the beginning of this article. It measures whether aspects of the PSD model are perceived by the users of technology. These aspects are: perceived primary task support, perceived dialogue support, perceived credibility, perceived social support, perceived unobtrusiveness, perceived persuasiveness, perceived effort, perceived effectiveness and use continuance [5]. The PPQ has already been applied in various studies to evaluate eHealth technology [6, 7]. However, the reliability and (construct) validity of the PPQ measures have not been elaborately studied yet.

In previous research, we performed an exploratory pilot evaluation of the PPQ based on user and expert evaluations [6]. Then, a pilot study was performed for the evaluation of the (construct) validation of the PPQ [8]. The current paper presents the results of a followup study, aimed at the evaluation of the (construct) validity of the PPQ. We applied a mixed-methods approach by combining a closed card sort task (with PPQ items and constructs) with a subjective evaluation of RunKeeper app (https://runkeeper.com/running-app) using the PPQ. In this study, we focus on the constructs and items of the PPQ: should these constructs be part of the PPQ and do these items fit the constructs they represent?

\section{Methods}

The current study uses a mixed-methods approach, combining (a) a closed-ended card sort [9] with (b) the subjective evaluation of a mobile eHealth application. A within-subject design was applied in this study. Participants performed tasks ( $a$ \& b) in a randomized order. Below, methods for the card sort (paragraph 2.2) and the evaluation of the eHealth application (paragraph 2.3) are described. 


\subsection{Participants}

Participants were selected via snowball and convenience sampling. The authors invited students of their departments to participate. A total of 65 university students (29 male, 36 female) participated in this study with various educational backgrounds (see Table 1). Of these, 19 were Finnish and 46 were Dutch students. Their mean age is 24.71 (ranging from 18 and 51). Most participants (89\%) were (somewhat or very) familiar with eHealth. Yet, some $(11 \%)$ participants indicate not being familiar with eHealth at all.

Table 1. Characteristics of the study participants $(N=65)$

\begin{tabular}{|c|c|c|c|}
\hline & $\mathrm{N}$ & $\begin{array}{l}\text { Mean } \\
\text { (Min.-Max.) }\end{array}$ & $\begin{array}{l}\text { Std. } \\
\text { Dev }\end{array}$ \\
\hline \multicolumn{4}{|l|}{ Nationality } \\
\hline Finnish & 19 & & \\
\hline Dutch & 46 & & \\
\hline \multicolumn{4}{|l|}{ Gender } \\
\hline Male & 29 & & \\
\hline Female & 36 & & \\
\hline Age & & $24.71(18-51)$ & 5.261 \\
\hline \multicolumn{4}{|l|}{ Scientific background } \\
\hline Behavioral, Management \& Social Science & 33 & & \\
\hline Information \& Communication Technology & 17 & & \\
\hline Science & & & \\
\hline Science \& Technology & 8 & & \\
\hline Electrical Engineering, Mathematics \& & 2 & & \\
\hline Computer Science & & & \\
\hline Other & 5 & & \\
\hline \multicolumn{4}{|l|}{ eHealth familiarity } \\
\hline Very & 25 & & \\
\hline Somewhat & 33 & & \\
\hline Not at all & 7 & & \\
\hline
\end{tabular}

\subsection{Card Sort}

For this part of the study, a closed card sort [9] was performed, using the items and constructs of the PPQ (see Appendix A for a complete overview of PPQ items and constructs). Originally, card sorting is a method aimed at evaluating or developing a system's information structure [9]. In this method, participants are asked to sort cards with excerpts of the system's content on it, into (for them) meaningful groups. The resulting sorts that participants make, provide insight into users' mental models, including (dis)agreement between users [7]. For the current study, the researchers made cards with PPQ items [5] on them, which users were asked to group into predefined categories (the PPQ constructs). The results allow us to determine the construct validity of the constructs of the PPQ.

\subsubsection{Materials}

Each participant was given a set of 31 white cards, each with a single PPQ item on it [10]. Also, nine colored cards were provided, each with a single PPQ construct and its definition on it. Participants had to group (white) item cards into the (colored) construct cards (see Figure 2). An overview of all PPQ items is given in Appendix A. 


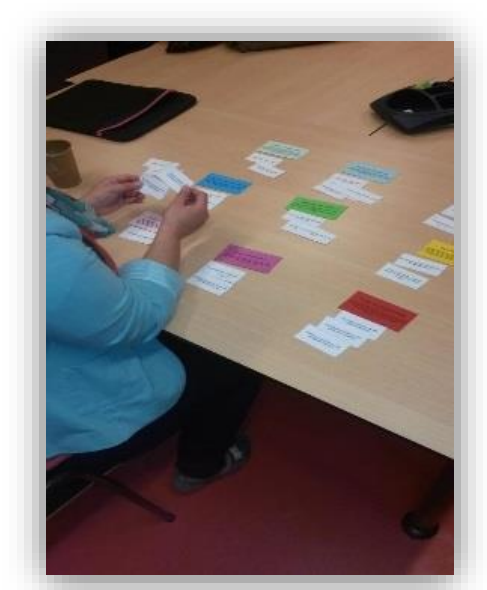

Figure 1. A participant is reading and sorting the PPQ cards during the card sorting task

\subsubsection{Procedure}

All participants were explained the purpose of the study and were asked to sign a consent form. To make sure that knowledge levels between participants was more or less equal, all participants were given a brief one-on-one PowerPoint presentation, explaining the constructs of the PPQ. Then, they were given the stack of item- and construct-cards. Participants were asked to group the items that they considered to be related, and to place them in one of the given construct-categories (See Figure 2). The only preconditions were that (1) all items had to be placed somewhere, and (2) all items had to be placed in no more than one construct. When the participant felt that all items were adequately grouped, the researcher took a picture of the sorted cards for data analysis.

\subsubsection{Data-analysis}

All card sorts were inserted into an Excel spreadsheet, that was developed for card sort analysis by Spencer [11], and was adapted for this study. The adapted version of the spreadsheet has been used in prior research as well [8, 12]. First of all, construct-level agreement was analyzed, to evaluate to what extend participants agreed on the content of each construct (the within construct agreement), using the equation:

Total amount of cards in the group / ( $n^{*}$ amount of unique cards in the group)

If little agreement was found, in-depth analysis on item-level was performed, by evaluating how many participants had grouped individual item in the intended construct.

For the items where participants reached little agreement on the construct they belong to, we explored how often any two items (irrespective of the construct they belong to) were paired together. Additionally, based on these data, a hierarchical cluster analysis (intervals based on squared Euclidian distance) was performed, using IBM SPSS 21.0. It was set to form between 2 and 31 clusters. First impressions of the clusters were based on the Agglomeration schedule. The clusters that were found, were cross-referenced (and if necessary: adjusted) based on (1) the Dendrogram and (2) the Icicle plot. 


\subsection{Evaluation of an eHealth app}

For the evaluation of an eHealth app, an app was sought that (a) stimulates a healthy lifestyle, (b) provides goal-setting support, (c) is available in Play Store and Apple store free of charge, and (d) is easily accessible for the study participants. Based on these criteria, RunKeeper application was selected for the user evaluation of an eHealth app. This app was chosen since it not only met our criteria and is a widely known fitness app.

\subsubsection{Materials}

The Dutch and Finnish participants all evaluated the RunKeeper app, which provides fitness coaching support (see Figure 2). Among various functionalities and features, the app allows a user to track his/her fitness activities using GPS. The user can choose his/her favorite fitness activity, plan a personal training scheme and also share recent fitness activities with friends via Facebook [13]. To ensure consistency, the Android (Google Play store) version of RunKeeper was used by all participants.

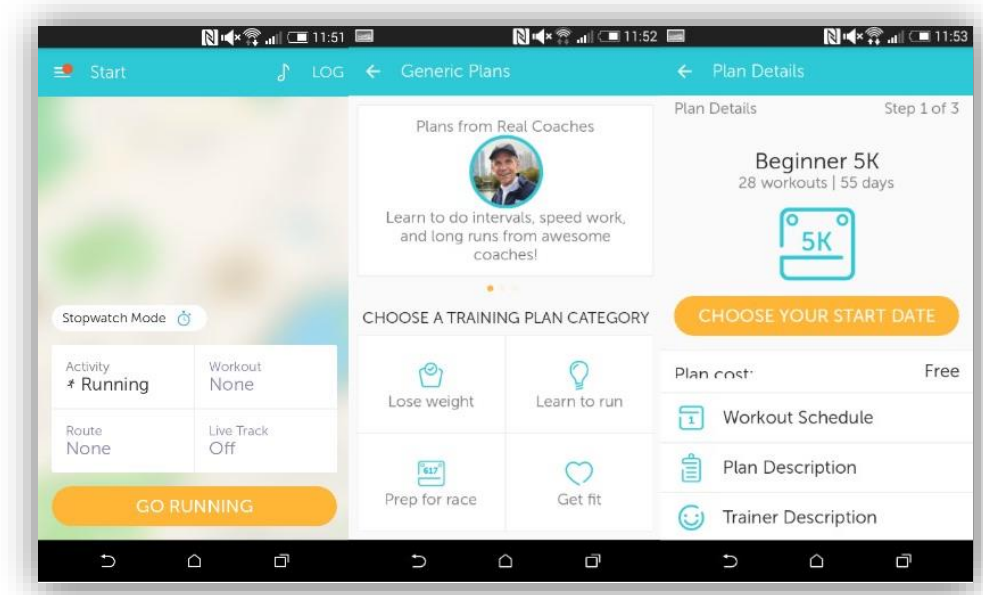

Figure 2 Screenshots of RunKeeper app (CFitnessKeeper ${ }^{1}$

Additionally, participants were handed a paper-based version of the PPQ. Herein, some items are recoded to include at least one reversed item per construct. The PPQ items were placed in random order to avoid bias. See Appendix A for a complete overview of PPQ items as they were used for the RunKeeper evaluation.

\subsubsection{Procedure}

To be able to fill in the PPQ, participants first had to get familiar with RunKeeper. Each participant was presented with a scenario-based task to simulate real-life user experience of the Runkeeper app. The task instructed the participants to choose their preferred sport activity (e.g., running, cycling) and to 'quick start' a $5 \mathrm{k}$ training schedule. After completing this task, participants were given the opportunity to freely explore the app by themselves. After that, participants were asked to score their appreciation of the app, via a paperbased version of the PPQ. This required them to score the $31 \mathrm{PPQ}$ items, on a 7-point Likert scale ranging from 1 (strongly agree) to 7 (strongly disagree). 


\subsubsection{Data-analysis}

For data-analysis, RunKeeper evaluation results were entered in IBM SPSS Statistics 21.0. Internal consistency of the items of the PPQ was rated by calculating Cronbach's Alpha based on RunKeeper evaluations. Additionally, Cronbach's Alpha when one item is removed was calculated.

\section{Results}

\subsection{Card Sort}

First of all, construct agreement was analyzed to gain insight into the degree to which participants agreed about which cards should belong to a construct (section 3.1.1). To look further into within-construct agreement, all items were also analyzed on item-level agreement (section 3.1.2). For the items that reached low agreement, it was evaluated how often two items were grouped together, regardless of (the name of) the construct they are intended to belong to. This was done via a cluster analysis (see section 3.1.3).

\subsubsection{Construct agreement}

The within-group construct agreement is rather low for all constructs. Social support scores the highest, but is still just 0.30 (see Table 2). This outcome is mainly useful when comparing with each other, not with a standard or norm. Thus, this finding requires further (in depth) analysis.

Table 2. Overview of within group agreement of card sort results

\begin{tabular}{lccc}
\hline Standardized category & Total cards* & Unique cards & Agreement \\
\hline Primary Task Support & 198 & 22 & 0.13 \\
\hline Perceived Dialogue Support & 189 & 25 & 0.11 \\
\hline Perceived Credibility Support & 265 & 22 & 0.18 \\
\hline Perceived Social Support & 201 & 10 & 0.30 \\
\hline Perceived Unobtrusiveness & 215 & 20 & 0.16 \\
\hline Perceived Persuasiveness & 223 & 29 & 0.11 \\
\hline Perceived Effort & 195 & 22 & 0.13 \\
\hline Perceived Effectiveness & 256 & 27 & 0.14 \\
\hline Use Continuance & 271 & 22 & 0.18 \\
\hline
\end{tabular}

${ }^{*}$ A single card may be included multiple times, by different participants.

\subsubsection{Item-level agreement}

The agreement per construct varied strongly (see Table 3). In its current form; each item of the PPQ theoretically belongs to just one of the constructs. However, the card sort revealed relatively broad spread of constructs where items are grouped.

Agreement on sixteen of the items was high (70-100\% of participants placed the item in the intended construct) (see Table 3). This goes for items 18 (perceived dialogue support), 4, 10, 19, 27 (perceived credibility), 21, 28, 30 (entire perceived social support construct), 6, 14 (perceived unobtrusiveness), 2 (perceived effort), 29 (perceived effectiveness), 7, 8, 13, 22 (entire use continuance construct).

Seven other items reached less, but still acceptable agreement (50-70\% of participants placing an item in the intended construct). These are 26 (perceived primary task support), 
11, 17 (perceived dialogue support), 24 (perceived unobtrusiveness), 31 (perceived effort), 3, 29 (perceived effectiveness).

The remaining nine items reached unsatisfactory $(<50 \%)$ agreement: 15, 23 (perceived primary task support), 16 (perceived credibility support), 1 (perceived unobtrusiveness), 5, 20, 25 (entire perceived persuasiveness construct), 9 (perceived effort), 12 (perceived effectiveness).

Table 3. Percentage of participants that grouped an item within a construct

\begin{tabular}{|c|c|c|c|c|c|c|c|c|c|c|}
\hline \multirow[t]{2}{*}{ 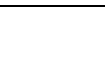 } & \multirow[t]{2}{*}{ Item } & \multicolumn{9}{|c|}{ Category of the PPQ } \\
\hline & & TASK & DIAL & CRED & $\mathrm{SOCl}$ & UNOB & PERS & EFFO & EFFE & CONT \\
\hline \multirow[t]{3}{*}{ TASK } & 15 & $31 \%$ & $5 \%$ & $2 \%$ & & $2 \%$ & $22 \%$ & $5 \%$ & $31 \%$ & $5 \%$ \\
\hline & 23 & $34 \%$ & $9 \%$ & & & $5 \%$ & $15 \%$ & $3 \%$ & $31 \%$ & $3 \%$ \\
\hline & 26 & $51 \%$ & $17 \%$ & & & $3 \%$ & $12 \%$ & $2 \%$ & $15 \%$ & \\
\hline \multirow[t]{3}{*}{ DIAL } & 11 & $12 \%$ & $51 \%$ & $3 \%$ & $3 \%$ & & $23 \%$ & & $6 \%$ & $2 \%$ \\
\hline & 17 & $14 \%$ & $57 \%$ & $9 \%$ & $12 \%$ & & $6 \%$ & & $2 \%$ & \\
\hline & 18 & $12 \%$ & $80 \%$ & & $2 \%$ & & $2 \%$ & $2 \%$ & $3 \%$ & \\
\hline \multirow[t]{5}{*}{ CRED } & 4 & $5 \%$ & $2 \%$ & $77 \%$ & & & $11 \%$ & $2 \%$ & $3 \%$ & $2 \%$ \\
\hline & 10 & $3 \%$ & $2 \%$ & $83 \%$ & & & $5 \%$ & $2 \%$ & $3 \%$ & $3 \%$ \\
\hline & 16 & $5 \%$ & $9 \%$ & $29 \%$ & $3 \%$ & & $23 \%$ & $9 \%$ & $20 \%$ & $2 \%$ \\
\hline & 19 & & $3 \%$ & $86 \%$ & & $2 \%$ & $3 \%$ & $2 \%$ & $5 \%$ & \\
\hline & 27 & $2 \%$ & & $88 \%$ & & $2 \%$ & $9 \%$ & & & \\
\hline \multirow[t]{3}{*}{$\mathrm{SOCl}$} & 21 & & $2 \%$ & & $98 \%$ & & & & & \\
\hline & 28 & & $5 \%$ & & $92 \%$ & $2 \%$ & $2 \%$ & & & \\
\hline & 30 & & $3 \%$ & $2 \%$ & $91 \%$ & & $3 \%$ & & $2 \%$ & \\
\hline \multirow[t]{4}{*}{ UNOB } & 1 & $12 \%$ & & & & $37 \%$ & $8 \%$ & $26 \%$ & $9 \%$ & $8 \%$ \\
\hline & 6 & $2 \%$ & & & & $86 \%$ & $5 \%$ & $3 \%$ & & $5 \%$ \\
\hline & 14 & $2 \%$ & & $2 \%$ & & $83 \%$ & & $9 \%$ & $2 \%$ & $3 \%$ \\
\hline & 24 & & $3 \%$ & $2 \%$ & & $57 \%$ & $2 \%$ & $31 \%$ & $2 \%$ & $5 \%$ \\
\hline \multirow[t]{3}{*}{ PERS } & 5 & $12 \%$ & $3 \%$ & $3 \%$ & $5 \%$ & $9 \%$ & $29 \%$ & $5 \%$ & $25 \%$ & $9 \%$ \\
\hline & 20 & $6 \%$ & $9 \%$ & $2 \%$ & & $2 \%$ & $42 \%$ & $3 \%$ & $34 \%$ & $3 \%$ \\
\hline & 25 & $17 \%$ & $12 \%$ & $2 \%$ & & $3 \%$ & $32 \%$ & $5 \%$ & $28 \%$ & $2 \%$ \\
\hline \multirow[t]{3}{*}{ EFFO } & 2 & $3 \%$ & & & & $8 \%$ & $3 \%$ & $78 \%$ & $2 \%$ & $5 \%$ \\
\hline & 9 & $18 \%$ & $3 \%$ & $6 \%$ & & $15 \%$ & $8 \%$ & $45 \%$ & $2 \%$ & $3 \%$ \\
\hline & 31 & $17 \%$ & $2 \%$ & $2 \%$ & & $11 \%$ & $2 \%$ & $63 \%$ & $2 \%$ & $3 \%$ \\
\hline \multirow[t]{3}{*}{ EFFE } & 3 & $19 \%$ & $3 \%$ & $3 \%$ & $2 \%$ & & $11 \%$ & $3 \%$ & $56 \%$ & $3 \%$ \\
\hline & 12 & $18 \%$ & $6 \%$ & $2 \%$ & & $2 \%$ & $43 \%$ & & $28 \%$ & $2 \%$ \\
\hline & 29 & $11 \%$ & & $5 \%$ & & & $9 \%$ & $2 \%$ & $74 \%$ & \\
\hline \multirow[t]{4}{*}{ CONT } & 7 & & $2 \%$ & $2 \%$ & & $2 \%$ & $2 \%$ & $2 \%$ & $5 \%$ & $88 \%$ \\
\hline & 8 & & $2 \%$ & & $2 \%$ & $2 \%$ & $6 \%$ & & $2 \%$ & $88 \%$ \\
\hline & 13 & & $2 \%$ & $2 \%$ & & $2 \%$ & $2 \%$ & & $2 \%$ & $92 \%$ \\
\hline & 22 & & $2 \%$ & & & & $6 \%$ & $2 \%$ & $6 \%$ & $85 \%$ \\
\hline
\end{tabular}




\subsubsection{Cluster analysis}

An overview of the groupings that were made is given in Table 4 . There was a big difference in how consistently cards were placed. The range of pairs that were made with the items that were found to have low-agreement was very high, being paired with 27 up to all 30 other items. However, the cluster analysis did provide clear insights into the data. Originally, the PPQ consists of nine constructs, whereas via construct analysis 'only' seven constructs were found (see Table 4, Table 6 and Appendices A \& B). Six of these were very clear (see Table 6) and almost perfectly resembled the original PPQ constructs perceived effort, perceived credibility, perceived unobtrusiveness, use continuance, perceived dialogue support and perceived social support. One of the clusters that was found during the cluster analysis, consists of three of the original PPQ constructs: perceived task support, perceived effectiveness and perceived persuasiveness.

Table 4: Outcomes of cluster analysis

\begin{tabular}{llcccc}
\hline Cluster & Original construct & \multicolumn{5}{c}{ Card numbers within the construct } \\
\hline 1 & Perceived effort (+ item 1) & 2 & 31 & 9 & 1 \\
\hline 2 & Perceived social support & 21 & 28 & 30 & \\
\hline 3 & Perceived credibility (- item 16) & 4 & 10 & 19 & 27 \\
\hline 4 & Perceived unobtrusiveness (- item 1) & 6 & 14 & 24 & \\
\hline 5 & Use continuance & 7 & 8 & 13 & 22 \\
\hline 6 & Perceived dialogue support & 11 & 17 & 18 & \\
\hline 7 & Perceived primary task support; Perceived & 3 & 5 & 12 & 15 \\
& persuasiveness; Perceived effectiveness & 16 & 20 & 23 & 25 \\
& (+ item 16) & 26 & 29 & & \\
\hline
\end{tabular}

The inter-item correlations within the clusters that were found via the cluster analysis varied (see Table 5). Three of the new found clusters reached high inter-item correlations: perceived social support, perceived credibility, use continuance and perceived unobtrusiveness. These were all clusters that were more or less similar in cluster analysis and original PPQ. The other clusters still reached rather lower levels of inter-item correlations, with the composite cluster (consisting of 10 items) reaching the lowest correlations (.385).

Table 5. Agreement on items within clusters that were found in the cluster analysis

\begin{tabular}{lcc}
\hline Cluster name & $\begin{array}{c}\text { Inter-item } \\
\text { correlations }\end{array}$ & $\begin{array}{c}\text { \# items in } \\
\text { the cluster }\end{array}$ \\
\hline Perceived effort + item 1 & .658 & 4 \\
\hline Perceived social support & .996 & 3 \\
\hline Perceived credibility (- item 16) & .967 & 4 \\
\hline Perceived unobtrusiveness (- item 1) & .891 & 3 \\
\hline Use continuance & .986 & 4 \\
\hline Perceived dialogue support & .637 & 3 \\
\hline Perceived primary task support; Perceived persuasiveness; & .385 & 10 \\
Perceived effectiveness (+ item 16) & & \\
\hline
\end{tabular}


Table 6. Output of the cluster analysis in Excel spreadsheet

\begin{tabular}{|c|c|c|c|c|c|c|c|c|c|c|c|c|c|c|c|c|c|c|c|c|c|c|c|c|c|c|c|c|c|c|c|}
\hline em & 1 & 2 & 9 & 31 & 3 & 5 & 12 & 15 & 16 & 20 & 23 & 25 & 26 & 29 & 4 & 10 & 19 & 27 & 6 & 14 & 24 & 7 & 8 & 13 & 22 & 11 & 17 & 18 & 21 & 28 & 30 \\
\hline 1 & & 30 & 39 & 30 & 6 & 12 & 10 & 9 & 9 & 10 & 13 & 9 & 9 & 9 & 3 & 6 & 6 & 6 & 36 & 31 & 33 & 10 & 7 & 9 & 10 & 7 & 3 & 4 & 3 & 4 & 3 \\
\hline 2 & 30 & & 42 & 57 & 4 & 6 & 7 & 9 & 15 & 9 & 7 & 7 & 3 & 7 & 4 & 6 & 3 & 4 & 9 & 15 & 30 & 10 & 7 & 6 & 6 & 6 & 3 & 4 & 3 & 4 & 3 \\
\hline 9 & 39 & 42 & & 57 & 7 & 13 & 7 & 12 & 12 & 6 & 18 & 3 & 12 & 4 & 9 & 10 & 7 & 6 & 19 & 19 & 16 & 6 & & 6 & 6 & 6 & 7 & & & & \\
\hline 31 & 30 & 57 & 57 & & 6 & 7 & 3 & 7 & 9 & 6 & 9 & 4 & 12 & 4 & 7 & 7 & 6 & 3 & 12 & 12 & 21 & 6 & 6 & 4 & 4 & 6 & 4 & & 3 & 3 & \\
\hline 3 & 6 & 4 & 7 & 6 & & 21 & 22 & 31 & 18 & 25 & 31 & 24 & 25 & 57 & 3 & 6 & 6 & 7 & 4 & 7 & 6 & 9 & 6 & 7 & 4 & 15 & 13 & 6 & 4 & 4 & \\
\hline 5 & 12 & 6 & 13 & 7 & 21 & & 28 & 27 & 18 & 24 & 18 & 22 & 18 & 25 & & 9 & 9 & 6 & 13 & 12 & 12 & 10 & 15 & 12 & 12 & 15 & 9 & & 7 & 7 & \\
\hline 12 & 10 & 7 & 7 & 3 & 22 & 28 & & 36 & 22 & 33 & 31 & 40 & 22 & 40 & 7 & 6 & 6 & 7 & 7 & 7 & 7 & 6 & 6 & & 4 & 22 & 12 & 13 & & & \\
\hline 15 & & & & & 31 & 27 & 36 & & 16 & 25 & 40 & 25 & 25 & 34 & 6 & 7 & 4 & 4 & 4 & 6 & 7 & 10 & 10 & 7 & 4 & 12 & 6 & 10 & 3 & 3 & \\
\hline 16 & & Perce & eived & & 18 & 18 & 22 & 16 & & 24 & 13 & 16 & 9 & 21 & 25 & 31 & 28 & 30 & 3 & 4 & 7 & 7 & 3 & 4 & 6 & 18 & 15 & 15 & 6 & 7 & \\
\hline 20 & 1 & & fort & & 25 & 24 & 33 & 25 & 24 & & 28 & 39 & 16 & 31 & 6 & 7 & 4 & 9 & 4 & 7 & 7 & 10 & & 6 & 9 & 30 & 9 & 13 & & & \\
\hline 23 & 1 & + ite & em 1 & & 31 & 18 & 31 & 40 & 13 & 28 & & 30 & 28 & 31 & 7 & 6 & 3 & 4 & 7 & 7 & 9 & 7 & 6 & 4 & 3 & 10 & 10 & 10 & 3 & 4 & \\
\hline 25 & & & & 4 & 24 & 22 & 40 & 25 & 16 & 39 & 30 & & 16 & 33 & 9 & 4 & 4 & & & & & 0 & 9 & 6 & 6 & 22 & 12 & 13 & 3 & 4 & \\
\hline 26 & 9 & 3 & 12 & 12 & 25 & 18 & 22 & 25 & 9 & 16 & 28 & 16 & & 19 & 7 & 4 & 3 & 4 & \multirow{3}{*}{\multicolumn{4}{|c|}{$\begin{array}{l}\text { Perceived use } \\
\text { continuance }\end{array}$}} & & & 4 & 21 & 25 & 16 & & 3 & \\
\hline 29 & 9 & 7 & 4 & 4 & 57 & 25 & 40 & 34 & 21 & 31 & 31 & 33 & 19 & & 6 & 9 & 9 & 7 & & & & & & 9 & 7 & 7 & 3 & 3 & \\
\hline 4 & 3 & 4 & 9 & 7 & 3 & 4 & 7 & 7 & 25 & 6 & 7 & 9 & 7 & 6 & & 70 & 75 & 78 & & & & & & & & 7 & 12 & 4 & 3 & 3 & 4 \\
\hline 10 & 6 & 6 & 10 & 7 & 6 & 9 & 6 & 7 & 31 & 7 & 6 & 4 & 4 & 9 & 70 & & 73 & 85 & 3 & 4 & 6 & 9 & & & & 6 & 12 & 3 & 3 & 4 & 4 \\
\hline 18 & 6 & 3 & 7 & 6 & 6 & 9 & 6 & 4 & 28 & 4 & 3 & 4 & 3 & 9 & 75 & 73 & & 75 & 3 & & 6 & 4 & & & & & 16 & 4 & 3 & 3 & 4 \\
\hline 27 & 6 & 4 & 6 & 3 & 7 & 6 & 7 & 4 & 30 & 9 & 4 & 6 & 4 & 7 & 78 & 85 & 75 & & 4 & & & 4 & 3 & 6 & 4 & \multirow{4}{*}{\multicolumn{3}{|c|}{$\begin{array}{l}\text { Perceived } \\
\text { dialogue } \\
\text { support }\end{array}$}} & & 4 & 4 \\
\hline 6 & 36 & 9 & 19 & 12 & 4 & 13 & 7 & 4 & 3 & 4 & 7 & 7 & 4 & 3 & 3 & $\Omega$ & 3 & 4 & & 84 & 55 & 0 & 9 & 9 & 9 & & & & & 4 & 3 \\
\hline 14 & 31 & 15 & 19 & 12 & & In & 7 & & & & & 9 & 3 & 6 & 3 & 4 & 4 & 4 & 84 & & 61 & 7 & & 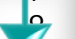 & 6 & & & & & 4 & 3 \\
\hline 24 & 33 & 30 & 16 & 21 & & Perc & eivec & prin & mary & task & & 10 & 4 & 0 & 3 & 6 & 6 & 4 & 55 & 61 & & 9 & 7 & & 6 & & & & & 4 & 3 \\
\hline 7 & 10 & 10 & 6 & 6 & & supp & ort, $p$ & persuc & asive & eness, & & 9 & 3 & & 7 & 0 & 1 & & 9 & 7 & 0 & & 82 & 87 & 79 & & 4 & 3 & \multirow{4}{*}{\multicolumn{3}{|c|}{$\begin{array}{l}\text { Perceived } \\
\text { social } \\
\text { support }\end{array}$}} \\
\hline 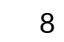 & 7 & 7 & 6 & 6 & & effec & tiven & ess + & + iten & m 16 & & 9 & 3 & & & Perce & eivec & & 9 & 6 & 7 & 82 & & 87 & 81 & 3 & & 3 & & & \\
\hline 13 & 9 & 6 & 6 & 4 & & & u & & & & & 6 & 3 & $t$ & & btrus & sivene & & 9 & 9 & 9 & 87 & 87 & & 90 & 4 & & 3 & & & \\
\hline 22 & 10 & 6 & 6 & 4 & 4 & 12 & 4 & 4 & 6 & 9 & 3 & 6 & 4 & $t$ & & - iter & & & 9 & 6 & 6 & 79 & 81 & 90 & & 7 & & 3 & & & \\
\hline 1 & 7 & 6 & 6 & 6 & 13 & 15 & 22 & 12 & 18 & 30 & 10 & 22 & 21 & c) & & & & & 3 & 4 & & 4 & 2 & 4 & & & 30 & 40 & 4 & & 4 \\
\hline 1 & 3 & 3 & 7 & 4 & 13 & 9 & 12 & 6 & 15 & 9 & 10 & 12 & 25 & 7 & 12 & 12 & 16 & 12 & 3 & & & & & & 3 & 30 & & 60 & 13 & 13 & 10 \\
\hline 1 & 4 & 4 & 6 & 7 & 6 & 6 & 13 & 10 & 15 & 13 & 10 & 13 & 16 & 7 & 4 & 3 & 4 & 3 & 3 & 3 & 4 & 3 & 3 & 3 & 3 & 40 & 60 & & 6 & $V$ & 7 \\
\hline 2 & 3 & 3 & 3 & 3 & 4 & 7 & 3 & 3 & 6 & 3 & 3 & 3 & 3 & 3 & & 3 & & & & & & & & 0 & & 4 & 13 & 6 & & 94 & 93 \\
\hline & 4 & 4 & 3 & 3 & 6 & 7 & 3 & 3 & 7 & 4 & 4 & 4 & 3 & 3 & 3 & 4 & & & & & & & & & & & 13 & & 94 & & 90 \\
\hline & 3 & 3 & 3 & 3 & 4 & 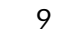 & 4 & 6 & 6 & 6 & & 3 & 4 & 4 & 4 & & & & & & & & & & & & 10 & & & 0 & \\
\hline
\end{tabular}

Darker cell color indicates higher percentage of participants placed two items together. 


\subsection{Evaluation of an eHealth app}

\subsubsection{Internal consistency of original PPQ constructs}

An overview of the Cronbach's Alpha values of all (original) PPQ constructs, including Alpha if one item removed, is given in Table 7. For almost half (44\%) of the constructs, the internal consistency was high; with Cronbach's Alpha being higher than 0.7. This implies the items of the constructs perceived credibility, perceived effort, perceived effectiveness and use continuance are coherent. The list of constructs with high internal consistency could be complemented with perceived dialogue support if one of its items (item 11 "RunKeeper encourages me").

Even for the constructs with high internal consistency, it is noticeable that their internal consistency could sometimes still be improved by removing one item. This goes for perceived credibility (without item 16 "RunKeeper does not provide confidence") and use continuance (without item 8 "I am considering discontinuing using RunKeeper"). The remaining four constructs (primary task support, perceived social support, unobtrusiveness and perceived persuasiveness) scored below the threshold for internal consistency, and remained that way if one item was removed.

Table 7. Overview of Cronbach's Alpha per original PPQ constructs

\begin{tabular}{|c|c|c|c|c|c|c|}
\hline Construct & $\begin{array}{l}\text { Cronbach's } \\
\text { Alpha }\end{array}$ & Items & Mean & SD & $\begin{array}{l}\text { Corrected item- } \\
\text { total correlation }\end{array}$ & $\begin{array}{l}\text { Cronbach's Alpha } \\
\text { if item deleted }\end{array}$ \\
\hline \multirow[t]{3}{*}{ TASK } & .682 & 15 & 3.89 & 1.572 & .484 & .602 \\
\hline & & 23 & 3.77 & 1.477 & .583 & .482 \\
\hline & & 26 & 4.23 & 1.647 & .429 & .678 \\
\hline \multirow[t]{3}{*}{ DIAL } & .654 & 11 & 4.28 & 1.281 & .343 & .723 \\
\hline & & 17 & 4.29 & 1.259 & .500 & .509 \\
\hline & & 18 & 4.89 & 1.134 & .572 & .424 \\
\hline \multirow[t]{5}{*}{ CRED } & .756 & 4 & 4.65 & 1.217 & .568 & .697 \\
\hline & & 10 & 4.72 & 1.125 & .558 & .703 \\
\hline & & 16 & 4.49 & 1.324 & .356 & .774 \\
\hline & & 19 & 3.88 & 1.409 & .517 & .717 \\
\hline & & 27 & 4.55 & 1.146 & .660 & .667 \\
\hline \multirow[t]{3}{*}{$\mathrm{SOCl}$} & .537 & 21 & 3.97 & 1.520 & .360 & .418 \\
\hline & & 28 & 5.43 & 1.185 & .296 & .518 \\
\hline & & 30 & 3.88 & 1.635 & .408 & .334 \\
\hline \multirow[t]{4}{*}{ UNOB } & .498 & 1 & 4.68 & 1.415 & .072 & .602 \\
\hline & & 6 & 4.34 & 1.564 & .418 & .297 \\
\hline & & 14 & 5.06 & 1.435 & .411 & .316 \\
\hline & & 24 & 4.69 & 1.510 & .294 & .424 \\
\hline \multirow[t]{3}{*}{ PERS } & .547 & 5 & 3.58 & 1.609 & .258 & .603 \\
\hline & & 20 & 3.95 & 1.484 & .440 & .321 \\
\hline & & 25 & 3.91 & 1.598 & .389 & .395 \\
\hline \multirow[t]{3}{*}{ EFFO } & .772 & 2 & 4.83 & 1.420 & .547 & .756 \\
\hline & & 9 & 4.83 & 1.387 & .712 & .586 \\
\hline & & 31 & 5.2 & 1.679 & .583 & .734 \\
\hline \multirow[t]{3}{*}{ EFFE } & .706 & 3 & 4.32 & 1.348 & .456 & .695 \\
\hline & & 12 & 4.08 & 1.584 & .595 & .521 \\
\hline & & 29 & 3.92 & 1.524 & .531 & .607 \\
\hline \multirow[t]{4}{*}{ CONT } & .863 & 7 & 3.29 & 1.934 & .806 & .783 \\
\hline & & 8 & 3.38 & 1.791 & .446 & .922 \\
\hline & & 13 & 3.57 & 1.904 & .794 & .789 \\
\hline & & 22 & 3.34 & 1.814 & .824 & .778 \\
\hline
\end{tabular}




\subsubsection{Internal consistency of 'new' PPQ clusters}

When performing an analysis of the internal consistency of the clusters that were formed via cluster analysis, Cronbach's Alpha values were higher (see Table 8). Now, four out of seven clusters (57\%) reached high internal consistency. This goes for the new cluster of Perceived Task Support + Perceived Persuasiveness + Perceived Effectiveness (+item 16 "RunKeeper does not provide confidence"), Perceived Credibility (-item 16 "RunKeeper does not provide confidence"), Perceived Effort (+item 1 "Using RunKeeper is practical / convenient for me") and Use Continuance. Again, this list could be complemented with Perceived Dialogue support if item 11 ("RunKeeper encourages me") were removed.

The other three clusters all did not reach the threshold for internal consistency. Additionally, none of these clusters would improve their internal consistency by removing an item.

Table 8. Overview of Cronbach's Alpha per 'new' PPQ cluster

\begin{tabular}{|c|c|c|c|c|c|c|}
\hline Cluster & $\begin{array}{l}\text { Cronbach's } \\
\text { Alpha }\end{array}$ & Items & Mean & SD & $\begin{array}{l}\text { Corrected } \\
\text { item-total } \\
\text { correlation }\end{array}$ & $\begin{array}{l}\text { Cronbach's } \\
\text { Alpha if item } \\
\text { deleted }\end{array}$ \\
\hline TASK + & .854 & 3 & 4.32 & 1.348 & .394 & .853 \\
\hline PERS + & & 5 & 3.58 & 1.609 & .366 & .857 \\
\hline \multirow[t]{8}{*}{ EFFE $(+16)$} & & 12 & 4.08 & 1.584 & .718 & .825 \\
\hline & & 15 & 3.89 & 1.572 & .572 & .839 \\
\hline & & 16 & 4.49 & 1.324 & .344 & .856 \\
\hline & & 20 & 3.95 & 1.484 & .657 & .831 \\
\hline & & 23 & 3.77 & 1.477 & .786 & .820 \\
\hline & & 25 & 3.91 & 1.598 & .638 & .833 \\
\hline & & 26 & 4.23 & 1.647 & .442 & .851 \\
\hline & & 29 & 3.92 & 1.524 & .676 & .830 \\
\hline \multirow[t]{3}{*}{ DIAL } & .654 & 11 & 4.28 & 1.281 & .343 & .723 \\
\hline & & 17 & 4.29 & 1.259 & .500 & .509 \\
\hline & & 18 & 4.89 & 1.134 & .572 & .424 \\
\hline \multirow[t]{4}{*}{ CRED $(-16)$} & .774 & 4 & 4.65 & 1.217 & .628 & .692 \\
\hline & & 10 & 4.72 & 1.125 & .513 & .751 \\
\hline & & 19 & 3.88 & 1.409 & .574 & .727 \\
\hline & & 27 & 4.55 & 1.146 & .607 & .706 \\
\hline \multirow[t]{3}{*}{$\mathrm{SOCl}$} & .537 & 21 & 3.97 & 1.520 & .360 & .418 \\
\hline & & 28 & 5.43 & 1.185 & .296 & .518 \\
\hline & & 30 & 3.88 & 1.635 & .408 & .334 \\
\hline UNOB & .602 & 6 & 4.34 & 1.564 & .357 & .584 \\
\hline \multirow[t]{2}{*}{$(-1)$} & & 14 & 5.06 & 1.435 & .472 & .416 \\
\hline & & 24 & 4.69 & 1.510 & .409 & .505 \\
\hline \multirow[t]{4}{*}{ EFFO (+ 1) } & .807 & 1 & 4.83 & 1.420 & .625 & .757 \\
\hline & & 2 & 4.68 & 1.415 & .592 & .772 \\
\hline & & 9 & 4.83 & 1.387 & .676 & .734 \\
\hline & & 31 & 5.20 & 1.679 & .612 & .769 \\
\hline \multirow[t]{4}{*}{ CONT } & .863 & 7 & 3.29 & 1.934 & .806 & .783 \\
\hline & & 8 & 3.38 & 1.791 & .446 & .922 \\
\hline & & 13 & 3.57 & 1.904 & .794 & .789 \\
\hline & & 22 & 3.34 & 1.814 & .824 & .778 \\
\hline
\end{tabular}

\section{Discussion}

This paper presents a first step towards the validation of the Perceived Persuasiveness Questionnaire (PPQ). For that purpose, a closed-ended card sort study and a user 
evaluation of an eHealth application via the PPQ were carried out. This paper has presented the authors with some answers and some new and interesting questions.

First of all, the card sort results have shown that construct-level agreement was low and that item-level agreement varied strongly. Performing a cluster analysis allowed for a more in-depth analysis of the results. It demonstrated that the items that originally were divided into nine constructs of the PPQ were now clustered into seven clusters. PPQ constructs that remained more or less unchanged were: Perceived Dialogue Support, Perceived Social Support, Perceived effort, Use Continuance, Perceived Credibility, and Perceived Unobtrusiveness. The last 'new' construct combines three of the original PPQ constructs (Perceived Primary Task Support, Perceived Persuasiveness, and Perceived Effectiveness) into one, complemented with item 16 "RunKeeper does not provide confidence". It seems that, according to the participants the in the latter 'new' construct items are all focused on how well (they feel that) the technology supports them in working towards their goals. Therefore, we suggest naming this construct 'Perceived Goal Support'.

Overall, it is noticeable that the above mentioned new clusters also scored higher on internal consistency (measured based on the subjective evaluation of RunKeeper, via Cronbach's Alpha). Thus, the results of the different methods that were used in this study are consistent as well. This is an indication that results seem to be robust, at least for these two methods.

An additional consideration that should be taken into account for further evaluation and validation of the PPQ, is whether the PPQ constructs cover similar entities. For example, as of yet, some of the constructs / items seem to be outcome- (e.g., perceived effectiveness) and others for example usability- (e.g., perceived persuasiveness) oriented. It should be discussed whether it is useful, or even desirable, to measure such differing entities within a single questionnaire. Additionally, the results of the current study have shown that the participants struggled with the construct perceived persuasiveness during the card sort study. They were unsure how to sort its cards. Looking at the PPQ items, it is noticeable that Perceived Persuasiveness is focused on the users' attitude about the technology [14]. The other constructs, on the other hand, represent a particular aspect of a technology. For example, they are focused on the technology's reliability or the extent to which it enables social interactions with peers. All such different aspects of the technology together determine the users' attitude towards it. So actually, it seems that the Perceived Persuasiveness is the sum of all the other constructs, which means that it should not be a construct in itself.

Moreover, it is questionable whether all constructs of the PSD model are adequately covered in the PPQ as it is now. One of the great strengths of the PSD model, is that next to its theoretical foundation, it offers 28 design principles which allow for a more practical use of the model. Yet, by far not all of these design principles are reflected in the PPQ. At the same time, it should be noted that the PSD model is intended to provide technology designers (experts) a guideline for the incorporation of persuasive elements, whereas the $P P Q$ is aimed at measuring the users' (non-experts) experience. So, based on the results of our study, we opt to cover the PSD model on construct level in the PPQ, inquiring about each construct through two or three very similar questions. If it is possible, the PSD constructs should be redefined and even clearer described. This would allow researchers to measure them with just a few items that really ask the same thing (e.g., "are you supported in reaching your goals?"). This makes the questionnaire more concise, and prevents researchers from having to extensively cover the entire scope of a construct. 
As mentioned before, the PSD model is aimed at offering technology designers a guideline for applying persuasive features. The intention of doing so should be to strive towards reaching positive behavior change. However, as Stibe \& Cugelman [15] describe: not all interventions lead to positive results. In fact, they may unintentionally cause negative outcomes (labeled: 'backfiring'). The PPQ can contribute to preventing such backfiring of a technology, by continuously (both during development and after implementation) evaluate whether aspects of persuasion are experienced.

For future research, we strongly believe that it would be greatly beneficial for researchers to have a valid evaluation tool to measure perceived persuasiveness of different design elements of persuasive technology available. However, further validation of the PPQ is required, including cross comparisons of PPQ evaluation results with different (kinds of) persuasive technology.

Next step towards further validation of PPQ will be to take a step back and determine how to best cover and measure PSD constructs. Important choices will have to be made: should constructs be measured by few similar questions, or by covering the entire spectrum of the construct? After doing so, an item bank should be created to find suitable questions to evaluate the perceived persuasiveness of eHealth technology.

With the Perceived Persuasiveness Questionnaire in its current form (consisting of 31 items), it is not feasible to perform a Factor Analysis with 65 respondents. At least 153 participants would be needed to be able to adequately perform such analysis. For future research, it is advisable and our intention, to perform a more thorough Factor Analysis of the PPQ in its current form, or in a yet to be developed version, with a sufficient number of participants.

\section{Conclusion}

The PPQ is a promising and much needed tool to evaluate whether the users of a technology experience its persuasive design elements. However, it still requires further work in order to reach its full potential. The current study, applying a card sort and a subjective evaluation of an eHealth application, has offered valuable insight into some of the limitations of the PPQ as it is now. As of yet, internal consistency and construct validity vary strongly and are relatively low.

The authors suggest taking a step back, to determine the best way to cover the full scope and make the best use of the strengths of the PSD model in a single questionnaire.

\section{Acknowledgements}

The work that was performed by Nienke Beerlage-de Jong and Lisette van Gemert-Pijnen was funded by the Interreg IVa "EurSafety Health-net" project. 


\section{References}

1. Fogg, B.J., Persuasive Technology: Using computers to change what we think and do, 2003: Morgan Kaufmann Publishers.

2. Morrison, L.G., Yardley, L., Powell, J., et al., What Design Features Are Used in Effective e-Health Interventions? A Review Using Techniques from Critical Interpretive Synthesis. Telemedicine and E-Health, 2012, 18(2), p. 137-144.

3. Oinas-Kukkonen, H. and Harjumaa, M., Persuasive systems design: Key issues, process model, and system features. Communications of the Association for Information Systems, 2009, 24(1), p. 28.

4. Meschtscheriakov, A., Gärtner, M., Mirnig, A., et al., The Persuasive Potential Questionnaire (PPQ): Challenges, Drawbacks, and Lessons Learned, In: Persuasive Technology: 11 th International Conference (PERSUASIVE 2016), Springer International Publishing, 2016, p. 162-175.

5. Lehto, T., Oinas-Kukkonen, H., and Drozd, F., Factors Affecting Perceived Persuasiveness of a Behavior Change Support System. In: '33th International Conference on Information Systems, 2012, Association for Information Systems, Orlando, Florida.

6. De Jong, N., Wentzel, J., Kelders, S.M., et al., Evaluation of Perceived Persuasiveness Constructs by Combining User Tests and Expert Assessments. In: Second International Workshop on Behavior Change Support Systems, In conjunction with the 9th International Conference on Persuasive Technology, 2014, Padova, Italy.

7. Wentzel, J., Van Velsen, L., Van Limburg, M., et al., Participatory eHealth development to support nurses in antimicrobial stewardship. BMC medical informatics and decision making, 2014, 14(45).

8. Beerlage-de Jong, N., Kulyk, O., Wentzel, J., et al., 'Sorting out' the PPQ: A mixedmethods approach to evaluate Perceived Persuasiveness Questionnaire constructs, In: The 10th International Conference on Persuasive Technology (PERSUASIVE 2015), 2015, Chicago, Illinois, USA.

9. Rosenfeld, L. and Morville, P., Information architecture for the World Wide Web: Designing large-scale web sites. 2002: O'Reilly Media, Inc.

10. Lehto, T., and Oinas-Kukkonen, H., Explaining and Predicting Perceived Effectiveness and Use Continuance Intention of a Behavior Change Support System, Behaviour and Information Technology, 2014, 34(2), p. 176-189.

11. Spencer, D., Instructions for use: Card sort analysis spreadsheet, 2007, Maad Mob Interaction Design.

12. Wentzel, J., Müller, F., Beerlage-de Jong, N., et al., Card sorting to evaluate the robustness of the information architecture of a protocol website, International Journal of Medical Informatics, 2016, 86, p. 71-81.

13. RunKeeper (Version 6.6), 2015, FitnessKeeper: Massachusettes, USA.

14. Drozd, F., Lehto, T., and Oinas-Kukkonen, H., Exploring perceived persuasiveness of a behavior change support system: a structural model, In: International Conference on Persuasive Technology, Springer Berlin Heidelberg, 2012, p157-168.

15. Stibe, A. and Cugelman, B., Persuasive Backfiring: When Behavior Change Interventions Trigger Unintended Negative Outcomes. In: International Conference on Persuasive Technology, Springer Berlin Heidelberg, 2016, p. 65-77.

16. Fogg, B.J. and Nass, C., Silicon sycophants: the effects of computers that flatter. International Journal of Human-Computer Studies, 1997, 46(5), p. 551-561.

17. Klein, J., Moon, Y., and Picard, R.W., This computer responds to user frustration: Theory, design, and results, Interacting with computers, 2002, 14(2), p. 119-140.

18. Corritore, C.L., Kracher, B., and Wiedenbeck, S., On-line trust: concepts, evolving themes, a model. International Journal of Human-Computer Studies, 2003, 58(6), p. 737-758. 
19. Wathen, C.N. and Burkell, J., Believe it or not: Factors influencing credibility on the Web. Journal of the American society for information science and technology, 2002, 53(2), p. $134-144$.

20. Crano, W.D. and Prislin, R., Attitudes and persuasion. Annual Review of Psychology, 2006, 57, p. 345-374.

21. Petty, R. and Cacioppo, J.T., Communication and persuasion: Central and peripheral routes to attitude change, 2012: Springer Science \& Business Media.

22. Wood, W., Attitude change: Persuasion and social influence. Annual Review of Psychology, 2000, 51(1), p. 539-570.

23. Oinas-Kukkonen, $\mathrm{H}$. Requirements for measuring the success of persuasive technology applications. In: Proceedings of the 7th international conference on methods and techniques in behavioral research, 2010, ACM.

24. Oinas-Kukkonen, $\mathrm{H}$., A foundation for the study of behavior change support systems. Personal and Ubiquitous Computing, 2013, 17(6), p. 1223-1235.

25. Ayyagari, R., Grover, V., and Purvis, R., Technostress: technological antecedents and implications. MIS quarterly, 2011, 35(4), p. 831-858.

26. Hensel, B.K., Demiris, G., and Courtney, K.L., Defining obtrusiveness in home telehealth technologies. Journal of the American Medical Informatics Association, 2006, 13(4), p. 428-431.

27. Karahanna, E., Agarwal, R., and Angst, C.M., Reconceptualizing compatibility beliefs in technology acceptance research. MIS Quarterly, 2006, p. 781-804.

28. Bhattacherjee, A., Understanding information systems continuance: an expectationconfirmation model. MIS Quarterly, 2001, p. 351-370.

29. Lehto, T. and Oinas-Kukkonen, H., Explaining and predicting perceived effectiveness and use continuance intention of a behaviour change support system for weight loss. Behaviour \& Information Technology, 2015, 34(2), p. 176-189.

30. De Guinea, A.O. and Markus, M.L., Why break the habit of a lifetime? Rethinking the roles of intention, habit, and emotion in continuing information technology use. MIS Quarterly, 2009, p. 433-444.

31. Venkatesh, V., Morris, M.G., Davis, G.B., et al., User acceptance of information technology: Toward a unified view, MIS Quarterly, 2003, p. 425-478. 


\section{Appendices}

\section{Appendix A. Overview of PPQ items land item numbers]}

\begin{tabular}{|c|c|c|c|}
\hline Construct & Item & Item description & Source \\
\hline $\begin{array}{l}\text { Perceived } \\
\text { Primary Task } \\
\text { Support }\end{array}$ & $\begin{array}{l}15 \\
23 \\
26\end{array}$ & $\begin{array}{l}\text { Runkeeper application does not help me to start with exercising. } \\
\text { Runkeeper application helps me change my exercising habits. } \\
\text { Runkeeper application provides me with means to exercise. }\end{array}$ & {$[3,5]$} \\
\hline $\begin{array}{l}\text { Perceived } \\
\text { Dialogue } \\
\text { Support }\end{array}$ & $\begin{array}{l}11 \\
17 \\
18\end{array}$ & $\begin{array}{l}\text { Runkeeper application encourages me. } \\
\text { Runkeeper application provides me with appropriate counselling. } \\
\text { Runkeeper application provides me with appropriate feedback. }\end{array}$ & $\begin{array}{l}{[3,5} \\
16, \\
17]\end{array}$ \\
\hline $\begin{array}{l}\text { Perceived } \\
\text { Credibility }\end{array}$ & $\begin{array}{c}4 \\
10 \\
16 \\
19 \\
27\end{array}$ & $\begin{array}{l}\text { Runkeeper application shows expertise. } \\
\text { Runkeeper application is unreliable. } \\
\text { Runkeeper application does not provide confidence. } \\
\text { Runkeeper application is clearly made by health professionals. } \\
\text { Runkeeper application is trustworthy. }\end{array}$ & $\begin{array}{l}{[3,5} \\
18, \\
19]\end{array}$ \\
\hline $\begin{array}{l}\text { Perceived } \\
\text { Persuasive- } \\
\text { ness }\end{array}$ & $\begin{array}{c}5 \\
20 \\
25\end{array}$ & $\begin{array}{l}\text { Runkeeper application is not personally relevant for me. } \\
\text { Runkeeper application has an influence on me. } \\
\text { Runkeeper application makes me reconsider my exercising habits. }\end{array}$ & $\begin{array}{l}{[5,20-} \\
24]\end{array}$ \\
\hline $\begin{array}{l}\text { Unobtrusive- } \\
\text { ness }\end{array}$ & $\begin{array}{c}6 \\
14 \\
1 \\
24\end{array}$ & $\begin{array}{l}\text { Using Runkeeper application fits into my daily life. } \\
\text { Using Runkeeper application disrupts my daily routines. } \\
\text { Using Runkeeper application is practical / convenient for me. } \\
\text { Finding the time to use Runkeeper application is a problem for me. }\end{array}$ & $\begin{array}{l}{[3,5,} \\
25-27]\end{array}$ \\
\hline $\begin{array}{l}\text { Use } \\
\text { Continuance }\end{array}$ & $\begin{array}{c}7 \\
8 \\
13 \\
22\end{array}$ & $\begin{array}{l}\text { I am not going to use Runkeeper application from now on. } \\
\text { I am considering discontinuing using Runkeeper application. } \\
\text { I will be using Runkeeper application in the future. } \\
\text { I am going to continue using Runkeeper application. }\end{array}$ & $\begin{array}{l}{[5,28-} \\
30]\end{array}$ \\
\hline $\begin{array}{l}\text { Perceived } \\
\text { Effort }\end{array}$ & $\begin{array}{l}9 \\
2 \\
31\end{array}$ & $\begin{array}{l}\text { Using Runkeeper application is straightforward for me. } \\
\text { Using Runkeeper application does not require a lot of effort from } \\
\text { me. } \\
\text { Using Runkeeper application is difficult. }\end{array}$ & $\begin{array}{l}{[29,} \\
31]\end{array}$ \\
\hline $\begin{array}{l}\text { Perceived } \\
\text { Effectiveness }\end{array}$ & $\begin{array}{l}12 \\
3 \\
29\end{array}$ & $\begin{array}{l}\text { In my opinion, using Runkeeper application has an effect on my } \\
\text { willingness to exercise. } \\
\text { My chances of starting with exercising improve by using Runkeeper } \\
\text { application. } \\
\text { In my opinion, Runkeeper application has no effect on my } \\
\text { exercising habits / weight. }\end{array}$ & $\begin{array}{l}{[29,} \\
31]\end{array}$ \\
\hline $\begin{array}{l}\text { Perceived } \\
\text { Social } \\
\text { Support }\end{array}$ & $\begin{array}{l}21 \\
28 \\
30\end{array}$ & $\begin{array}{l}\text { I get support from my peers through Runkeeper application when I } \\
\text { need it. } \\
\text { Through Runkeeper application, I cannot share my experiences with } \\
\text { my peers. } \\
\text { Learning from my peers' actions is beneficial for me. }\end{array}$ & {$[3,29]$} \\
\hline
\end{tabular}


Appendix B. Icicle chart of item-level cluster analysis

Case

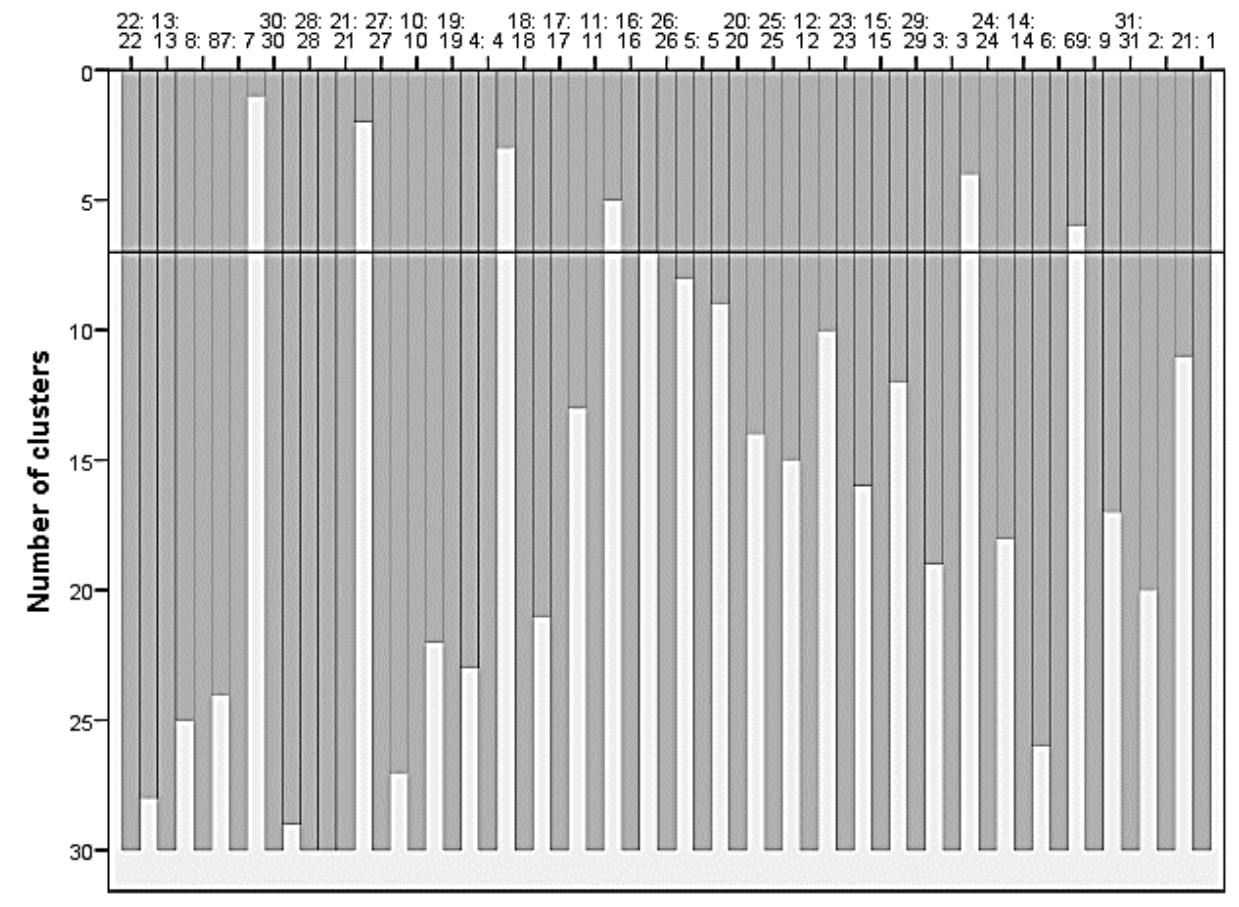




\section{Appendix C. Dendrogram of item-level cluster analysis}

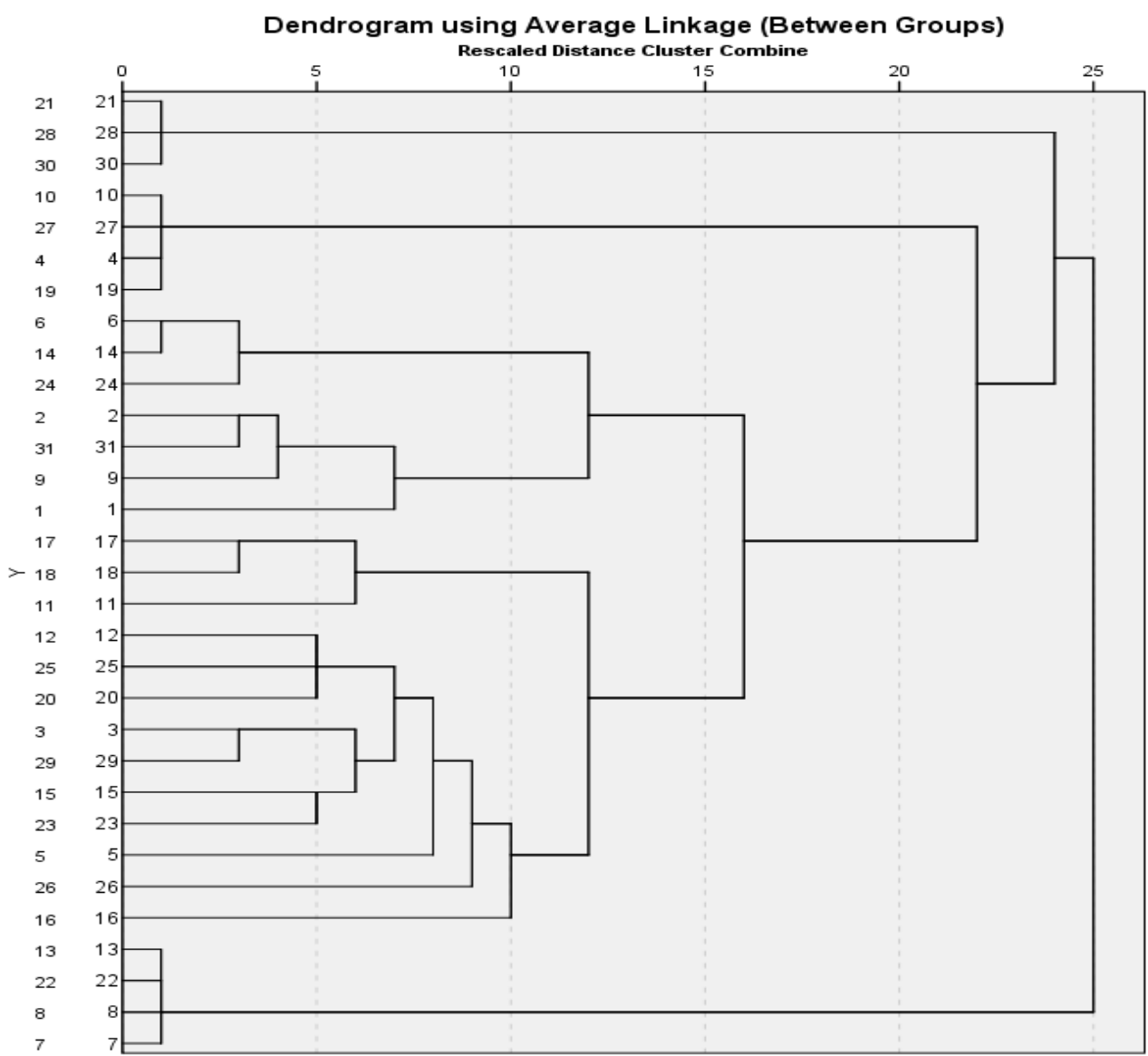





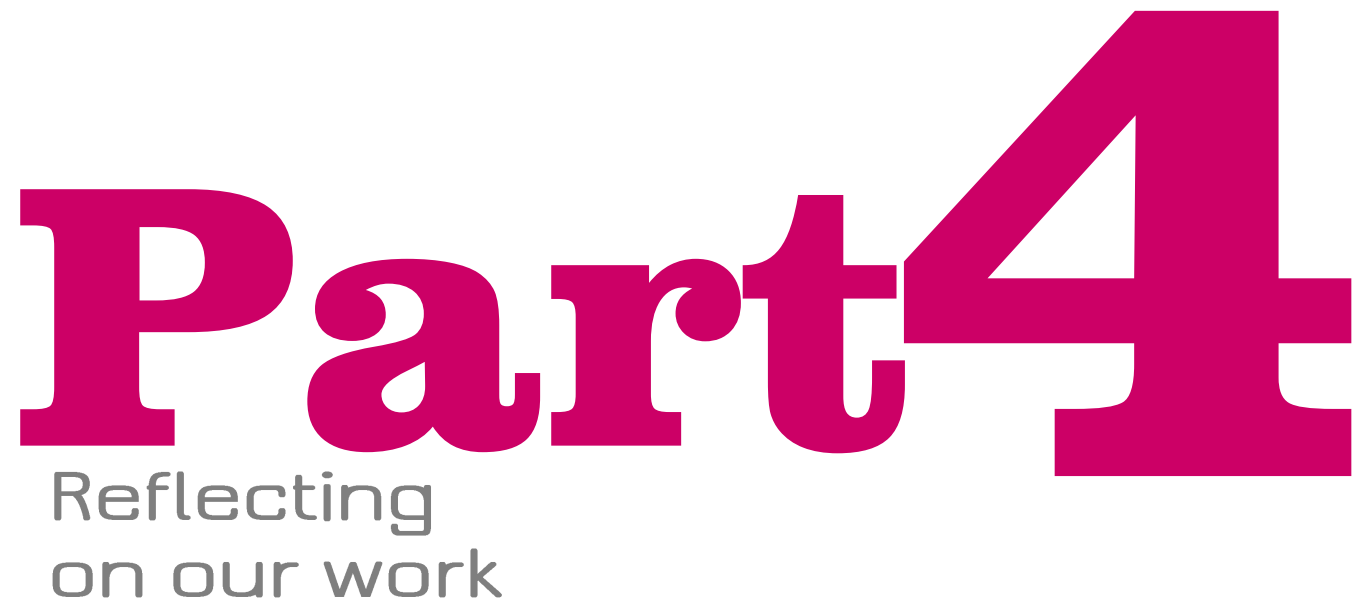





\section{Technology to support integrated Antimicrobial Stewardship Programs}

A user-centered and stakeholder driven development approach

N. Beerlage-de Jong, J. van Gemert-Piinen, J. Wentzel, R. Hendrix, L. Siemons Infectious Disease Reports (In Review) 


\section{Abstract}

The rise of antimicrobial resistance (AMR) is a severe global health problem. Combatting this problem requires the prudent prescribing of antimicrobials. This is promoted through Antimicrobial Stewardship Programs (ASPs). In this paper we describe 1) how a sociotechnical multidisciplinary approach (based on the CeHRes Roadmap) can be of value to support the development of Antimicrobial Stewardship technologies and 2) how these eHealth technologies can be implemented in practice.

The CeHRes Roadmap entails five different phases to explore and test how an eHealth technology can be tailored to the target group and successfully implemented in practice: 1) contextual inquiry, 2) value specification, 3) design, 4) operationalization, 5) evaluation. In this paper we describe the lessons learned from research and practice to guide future developments of technology based ASP interventions. Since AMR is a huge wicked problem on a global level, it requires innovative methods and models to empower general public and professionals to be proactive rather than reactive in a digitalized world. We highlight how to combat the dangerous rise of antimicrobial resistance in the future. 


\section{Introduction}

The rise of antimicrobial resistance (AMR) is a severe problem [1, 2]. Combatting this problem requires the prudent prescribing of antimicrobials. This is promoted through Antimicrobial Stewardship Programs (ASPs). These programs aim to monitor infections, implement safe work strategies, prevent the misuse of antimicrobials and educate healthcare workers. Although they are promising, they do not reach their full potential yet. Due to many factors, such as among others a lack of incentives, bad hygiene behaviours, unworkable regulations, lack of knowledge, and misuse of antimicrobials, our healthcare system becomes a breeding ground for antimicrobial resistance [2]. The mere fact that, in the USA alone over two million people become infected with multidrug resistant organisms each year, resulting in 23.000 deaths annually, and that costs of antimicrobial resistance are estimated at $\$ 20$ billion a year (USA), stresses the necessity to take action [3,4].

AMR is a huge "wicked" problem on a global level [5], with no single straightforward solution, requiring a so called theragnostic model based on infection prevention, antibiotic and diagnostic stewardship programs [6]. This multidisciplinary stewardship approach requires intensive collaboration among stakeholders [5, 7]. The current state-of-the-art is to not only combat superbugs with the development of new drugs, but to also find behavioral solutions in social sciences to create optimal conditions for the stewardship programs. The Centers for Disease Control and Prevention also stated that stewardship programs require a coordinated multidisciplinary approach and the continuous tracking of prescribing behaviors and resistance patterns, as well as information and education strategies to support healthcare workers (HCWs) [3]. This is where technology based behavioral solutions will help. With the introduction of technology in stewardship programs infection prevention, antibiotic prescribing and diagnostic interventions can be personalized to the needs of patients and healthcare workers [6].

In this paper we describe (1) how a socio-technical multidisciplinary approach (based on the CeHRes Roadmap) can be of value to support the development of Antimicrobial Stewardship technologies and (2) what has to be done to implement these technologies in practice. We describe the lessons learned from research and practice, to guide future developments of tech-based ASP technologies. In doing so, we elaborate on the report by the Centers for Disease Control and Prevention, which describes what interventions should be implemented in an ASP [3]. We complement this knowledge with insight in how to realize such interventions, through technology.

\section{The CeHRes Roadmap, a multidisciplinary development approach}

The CeHRes Roadmap was developed at the Centre for eHealth and Wellbeing Research of the University of Twente and introduced in the development of technologies for infection prevention and control in several projects (www.eursafety.eu; mrsa-net.nl) [8]. The Roadmap prioritizes the 'human standard' in the development of technologies for the improvement of healthcare, incorporating user-centered design principles, persuasive technology, and a stakeholder value development approach (business modeling). The integration of a user-centered design approach with a stakeholder-driven collaboration procedure during development enables a sustainable implementation of technologies in healthcare. In our view, the development of such technologies is more than creating a vehicle or tool to support people. It is a process of co-creation with end-users and other 
stakeholders to guarantee a fit between technology, users, and context (way of living and working). This co-creation process is coupled with continuous and iterative cycles of evaluation (formative evaluation) to gain insight in whether a technology can contribute to improving healthcare and what barriers hinder its implementation. Therefore, implementation is not a post design step, but is interwoven with the development of a technology. As such, eHealth development is not an ad hoc activity, but a structural approach to set the conditions for personalized healthcare using technology.

The Roadmap entails five different phases to explore and test how eHealth technology can be perfectly personalized and tailored to the target group and successfully implemented in practice (Figure 1).

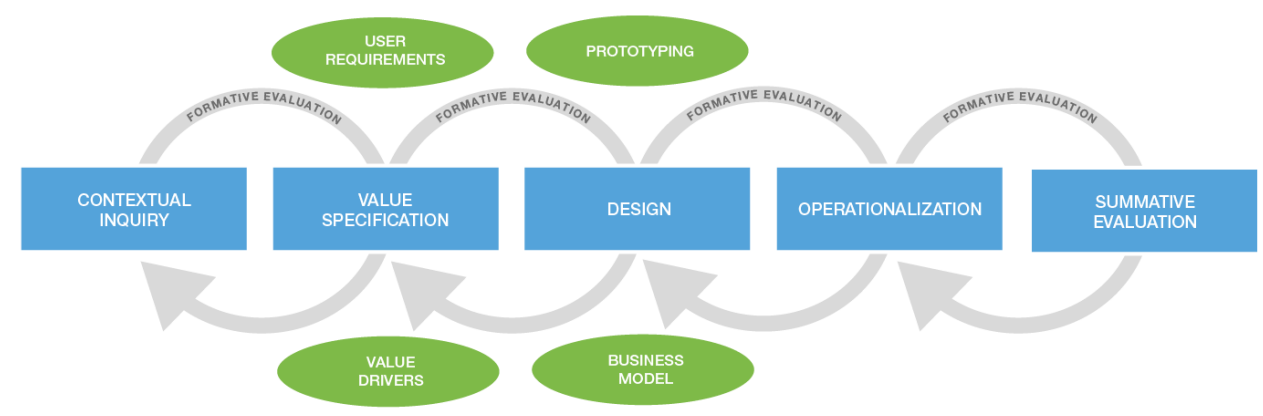

Figure 1. The CeHRes Roadmap

\section{Contextual inquiry}

The objective of the contextual inquiry phase is to identify the weak and strong points of the current situation, for example current Stewardship approaches to reduce AMR, to inquire the needs of stakeholders to improve health, how technology can be used to deal with the problems and needs, and to identify regulations and conditions that should be taken into account (i.e., legislation, regulations, policy) to implement an eHealth technology [9].

\section{Value specification}

The value specification phase builds on the results of the contextual inquiry. The identified key-stakeholders determine the added values (economical, medical, organizational etc.) that they want to achieve by means of a technical solution for the improvements in healthcare, such as a reduction of AMR. These values can be ranked in order to create a feasible technology and to set conditions for implementation (see also www.eHealthWiki. org) [10]. The values will be translated by the project management team (Research \& Development) into system, content and service requirements in a value map.

\section{Design}

Within this phase of the Roadmap, the design (the look-and-feel) of the technology is developed. In close cooperation with end-users (e.g., doctors, nurses, patients) several design-prototypes (from paper-based until fully functioning technology) can be developed and tested using scenarios that represent the context: the use of an AMR technology in healthcare practice. For the design of such prototypes, usability principles and persuasive design models (e.g., the Persuasive Systems Design Model) can be used to ensure a fit between technology, its use context and its users $[9,11,12]$. 


\section{Operationalization}

This phase entails activities to introduce a technology in healthcare. An implementation and a business model (e.g., timing, activities, actors, budget) is developed together with an evaluation plan to examine whether the goals, or values are realized [7].

\section{Evaluation}

To examine whether an eHealth technology works and helps, ongoing evaluation rounds (i.e., formative evaluation) are conducted throughout the whole developmental phase. Additionally, a final evaluation round (i.e., summative evaluation) is performed after implementation to measure the effects on behavior change, attitudes and the impact on the goals to be realized.

The CeHRes Roadmap is a guide that can be used integral to develop and implement technologies, as a guide to assess the developmental process or to guide a (partial) redesign process.

\subsection{Development of technology for Antimicrobial Stewardship Programs via the CeHRes Roadmap}

In this paper, we describe how the CeHRes Roadmap was used to develop several technologies to share information and to communicate with $\mathrm{HCWs}$, about how to prevent infections and how to avoid antimicrobial resistance. The CeHRes Roadmap has a good fit with complex problems like AMR and the integrated stewardship model because of its' multidisciplinary and socio technical based development approach [6]. We elaborate per ASP technology on a specific phase of the Roadmap, most suitable to deal with wicked problems and to focus on 'stewardship' supporting HCWs with technologies relevant for AMR. We describe a) a technology to support decision making about antimicrobials (focusing on Contextual Inquiry), b) a maturity model to guide the implementation of ASP technologies (Value specification \& Operationalization), c) a technology to support nurses in antimicrobial administration (Design to fit the use context), and d) a technology to register infections for nursing homes (Persuasive design). The technologies are available at www.infectionmanager.com, a platform in beta version to support Integrated AMR Stewardship.

\subsubsection{Technology to support decision making about antimicrobials [Contextual Inquiry]}

\section{Background}

Decision making in an ASP is a complex matter. Many factors influence a decision on how to diagnose and decide what treatment a patient needs. To support physicians in making decisions about the prescribing of antimicrobials, Clinical Decision Support Systems (CDSSs) are proposed and often implemented [13]. However, their success largely differs, mainly due to low use and low adherence rates. To increase the chances of success, CDSSs should fit its users' needs and expectations, but also their work processes.

\section{Activities}

We conducted a Contextual Inquiry to identify why and how a clinical decision system can support the prescribing of antimicrobials. A scoping review of the literature on existing CDSSs provided insight in their current development, design and implementation. It revealed a lack of involvement of potential end-users during the development, but also a 
lack of attention for the design process of CDSSs (most publications do not report on how a CDSS was developed). Additionally, existing CDSSs generally have a mainly clinical focus, they lack attention for stewardship and how to support decisions in practice. Insights from the scoping review were used to develop, test, and evaluate scenario-based prototypes with end users. Observing physicians while they interact with these prototypes, provided insight into existing work processes (e.g., current information sources), problems that end-users experience (e.g., indistinctness about duration of an AB therapy) and users' preferences and needs regarding the look and feel of the technology.

\section{Lessons learned}

- Early involvement of stakeholders and end-users is necessary to develop user friendly ASP technologies. Stakeholders, like experts in the field of infections and in other medical specialties, are needed to continuously keep an eye on the fit between technology and clinical practice, and to set conditions for implementation.

- Scenario-based prototype evaluations enable potential end-users to visualize and verbalize their work processes and problems they encounter, and to think outside their comfort zone, to discuss opportunities of technology based ASP.

- Scenario-based prototype evaluations (and cross-referencing its findings among other stakeholders) provide valuable information to harmonize and translate medically focused protocols and guidelines into practice based instructions.

- Involvement of end-users provides clear insight into improvements for the complex work environment that healthcare is. For example, currently most eHealth technology to support ASPs is directional, restrictive and less integrated with practice. Medical autonomy (which is a prerequisite for being able to deal with complex and ever changing circumstances) of e.g., physicians is sometimes neglected in ASP. End-users clearly indicated a need for a technology to respect and keep account with their medical autonomy, and to give room for deliberation (i.e., diagnostics as triage for decision making).

\subsubsection{Maturity model to implement Antimicrobial Stewardship Technologies [Value specification \& Operationalization]}

\section{Background}

Infection prevention and control should be considered as an integrated healthcare approach [6]. To determine what ASP interventions should be developed and to facilitate implementation, involvement of stakeholders is crucial. A maturity model can help to assess the activities already done and to guide the development of interventions, in cooperation with stakeholders such as medical professionals, infection experts, and (local) managers.

\section{Activities}

Business modeling activities were performed to identify the key stakeholders and to determine the values that have to be realized with ASPs in practice [14]. Multidisciplinary focus groups were conducted to decide what ASP technologies should be developed, to define their requirements, and to identify necessary resources and skills, from planning until operationalization of an ASP [15]. 


\section{Lessons learned}

- ASP interventions, supported by technology, can be bundled in a toolkit. Such ASP toolkit offers a set of technologies that are intended to support an integrated ASP approach [16];

- Information app to support antimicrobial prescribing (aimed at physicians)

- Interactive antimicrobial prescription decision support app

- Information app to support antimicrobial administration (in the Netherlands aimed at nurses)

- Information app to transfer patient care

- Tool to facilitate audits by the ASP-team

- Alerts/notifications

- Education via E-Learning

- Our maturity assessment checklist with questions regarding the status nascendi of local technologies can be used to decide the maturity of the ASP initiatives (e.g., done, planned);

- The maturity model that we developed guides the development of these technologies considering the aims, resources and capabilities of a local organization and (inter)national regulations for ASP. It presents the necessary information in manageable and relevant fragments to A-teams that are in the process of implementing ASP. That also makes it different from the few existing ASP guidelines or toolkits that exist [14].

In Figure 2 an overview of the maturity model for implementing ASP. Each button will have submenus with further implementation information. 


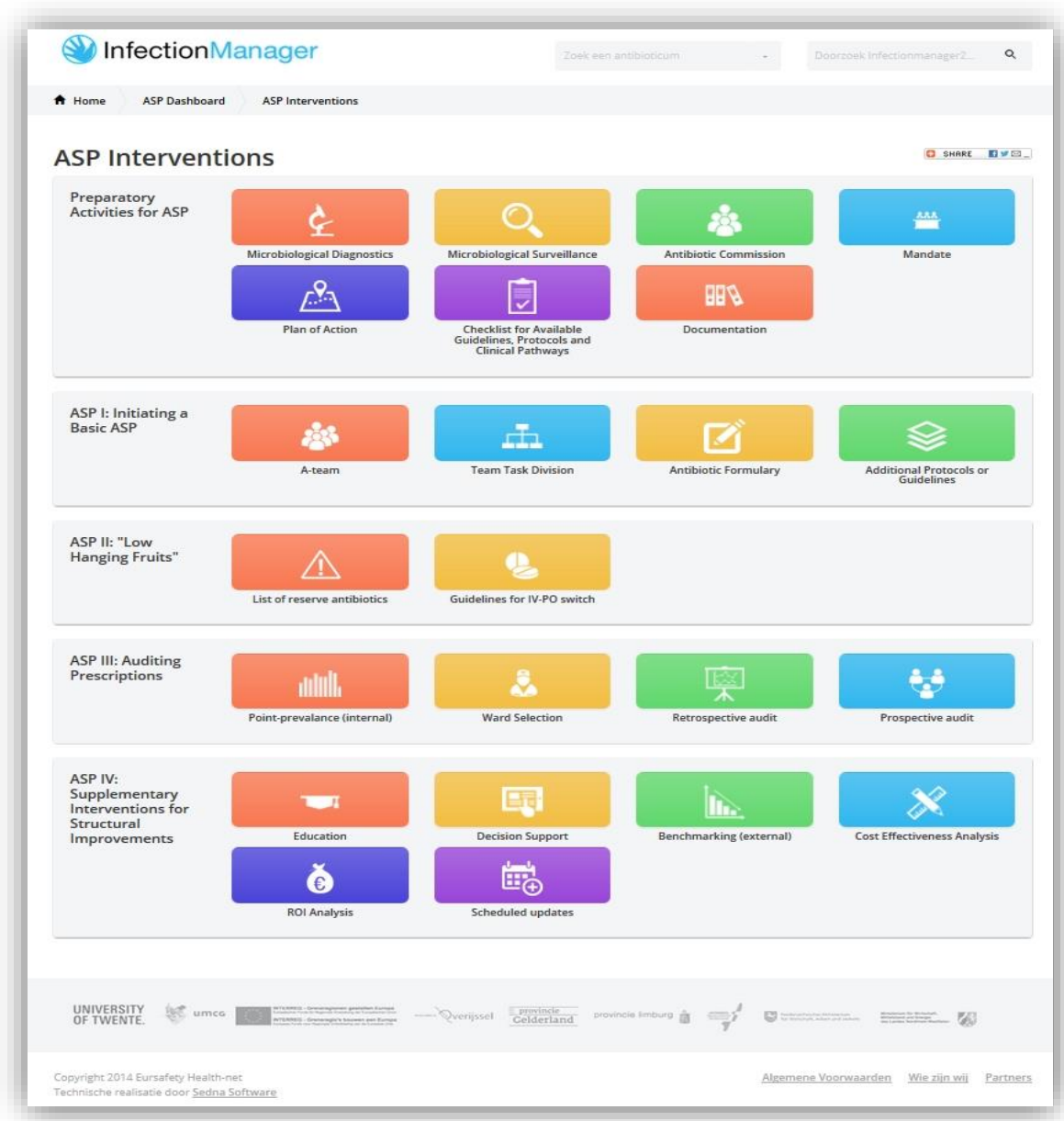

Figure 2. The Maturity model

\subsection{Technology to support nurses in antimicrobial administration [Design]}

\section{Background}

Optimal use of antimicrobials relies on careful prescription and administration. Often, the manuals and protocols that describe these complex activities are expert-driven, difficult to understand, inaccurate, or not in line with novel insights [17].

Nurses play a key role in administration and monitoring of antimicrobials. They inform physicians on the (actual) use and effects of antimicrobials on their patients, based on their daily care activities. This informative role is implicit in medical protocols. Yet, the cooperation between nurses and physicians in streamlining antimicrobial care for patients is crucial to successful ASPs [18]. 


\section{Activities}

Contextual inquiry indicated that nurses need information that supports them in their preparation, administration and monitoring tasks, and that assists them to observe possible risks or adverse events associated with antimicrobial therapy [17]. User-centered design principles with a focus on nurses' daily tasks were used to design prototypes of the Antibiotic Information app with nurses, doctors, microbiologist and pharmacists. To change the protocols into more usable and task-oriented information sources, card sorting was done. This method helps to create intuitive information structure for online information sources [19]. Nurses sorted cards with small chunks of information (e.g., parts of protocols, reference documents) in (what they regard as) logical groups. In addition, they named each group while thinking aloud. On the resulting sorts, a cluster analysis was performed to show how different information parts are related, according to the nurses, based on their experience. The results show that the app should present information in a taskoriented way, including practical instructions (e.g., on dose, preparation, administration and monitoring). In addition, background information (e.g., about the disease that requires antimicrobial treatment or specific characteristics of the antimicrobials) should be included (see also Figure 3).

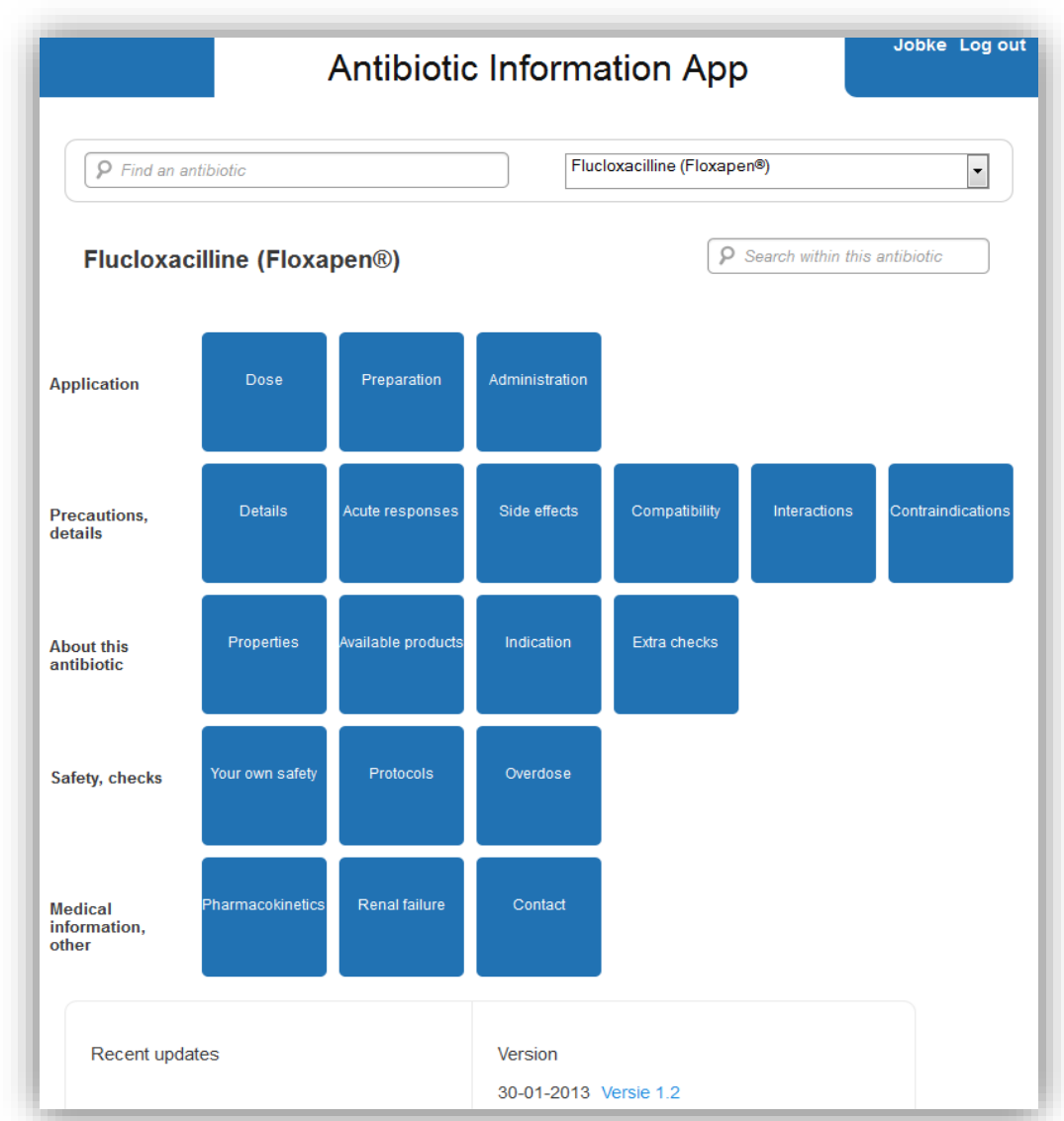

Figure 3. The Antibiotic Information app to support nurses in ASP 


\section{Lessons learned}

- To support the tasks of nurses, insight in information use is required.

- For technologies to be optimally supportive, explicit elicitation of and decisions on functional requirements (i.e., what the technology should be able to do) and design requirements (i.e., what interfaces should look like, how information should be structured to support tasks) is needed.

- Information should primarily be task oriented, but additional (in-depth) explanations and resources should be accessible as well, to reflect medical standards.

- Information availability is a prerequisite for active nurse involvement in ASP, but elearning is needed to stimulate novel practices.

\subsection{Technology to register infections in nursing homes [Design]}

\section{Background}

Once a year, nursing homes that are part of the (Dutch) iPrevent network register all their clients in a prevalence measurement of nursing home-associated infections and related risk factors (e.g., presence of catheters, antimicrobial use). This task is performed by elderly care physicians, who thus have to register numerous clients within a short period. The previous (paper-based) tool that was used to do so was time consuming, laborious and prone to interpretation errors [11]. To deal with these issues, a web-based registration and monitoring app for nursing home-associated infections (the Prevalence app) was developed.

To increase adherence it is important that technologies to support ASP, support its endusers in performing their tasks and should motivate them in doing so. Persuasive technology offers such support [12], to improve adherence with certain rules of conduct. Persuasive infection management is not focused on punishing bad compliance, as this has a demotivating effect, but on a positive, rewarding approach to behavioural change.

\section{Activities}

Scenario-based prototypes were evaluated by elderly care physicians (the intended endusers). For scenario-based user testing, a low-fidelity prototype of the Prevalence app was developed, incorporating elements of persuasive design. For example:

Primary Task Support was provided to simplify the tasks (e.g., reduction to shorten questions) and to make it less time consuming (e.g., tunnelling to omit irrelevant questions).

Dialogue Support was incorporated to avoid errors (e.g., suggestion to avoid typing errors, see Figure 4) and to make sure all relevant information is included (e.g., reminders to add laboratory test results as soon as they are available). 


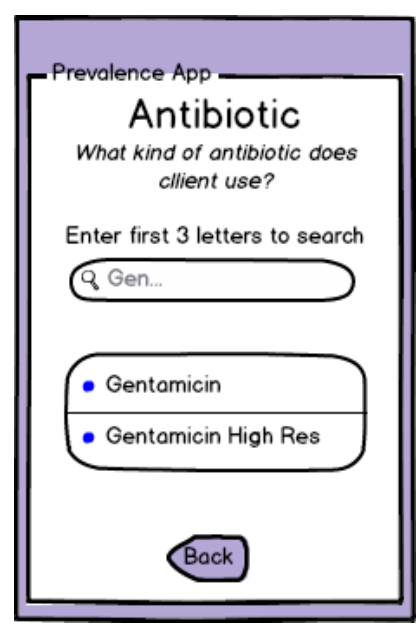

Figure 4. Example of Suggestion in a low-fidelity prototype

System Credibility Support was embedded to ensure its face validity (e.g., by clearly displaying the logo of the INTERREG IVa EurSafety Health-net project that it was developed in) and its reliability (e.g., by embedding the app in a larger website which provided additional information about the project and the experts who were involved in its development).

Social Support was used to allow nursing homes to reflect on their own infection rates (i.e., social Comparison to compare the own data that with a benchmark of the other participating nursing homes).

\section{Lessons learned}

- Persuasive technology matters. Preliminary evaluations showed that end-users performed (fictional, but real-life) tasks faster and with less mistakes than with the prior web-based system [20].

- Preliminary evaluations indicated that the perceived persuasiveness of the Prevalence app was higher than of the prior system [20].

- End-users expressed appreciation for the improved integration of working with the Prevalence app in their work processes. It enabled them to pause and continue registration at a later point in time, without losing data (as opposed to the prior system).

\section{Conclusion \& future work}

In this paper we have highlighted how technology can be used to contribute to fight antimicrobial resistance (AMR). AMR is a huge wicked problem on a global level, requiring innovative methods and models on a local level to empower our public and professionals to be proactive rather than reactive in a digitalized world. Technology supports the development of an integrated ASPs, crossing borders between healthcare and public health [5].

The CeHRes Roadmap provides the methodology to guide the ASP toolkit development process, providing methods for involving end-users (to create and test prototypes) and 
stakeholders (to define the added values and conditions for the implementation). The digitalization of information and the options for mobile technology facilitate an integrated and technology based Antimicrobial Stewardship Model [6]. Furthermore, ubiquitous digital networks offer facilities for continuous web-based education of the public, healthcare providers and infection prevention experts, making new knowledge easily available through eLearning modules [10].

\section{Future work}

The Centers for Disease Control and Prevention highlighted the key elements for ASP [3], we think technology can support the realization of the key elements by creating an infrastructure for infection prevention interventions and by continuous surveillance of antimicrobial use.

Automated surveillance systems have already been shown to be sensitive, specific and efficient to prevent the spread of Highly Resistant Micro Organisms (HRMO) and to reduce healthcare-associated infections [21-23]. However, current surveillance methods lack a systematic approach to detect changes or patterns in events that require acute interventions. Nor is surveillance currently used to predict antimicrobial resistance problems and upcoming outbreaks of diseases due to the lack of advanced analytics. Predictive analytics, using technologies to learn from historical data to make predictions about future health, are needed to enrich the current surveillance systems. Today an extensive growth in the amount of data in healthcare is digitally generated, collected, and stored via mobile and wearable technologies. These technologies provide opportunities to develop predictive, automated decision support systems, for public health and medical care settings. Using advanced algorithms in real-time modus and facilitating risk communication for proactive decision making in the prevention of HRMO outbreaks. We need to ensure there is a human standard in these developments, so that such information is not only available but also accessible and usable for health care workers, patients, citizens, and other stakeholders during their daily life or work. In current AMR projects (i.e., INTERREG Va Health-i-care, eZoon ZonMw) we use the ASP toolkit to develop an elearning environment and a web-based early warning decision support system to educate professionals and public, to reduce AMR. 


\section{References}

1. MacDougall, C. and Polk, R.E., Antimicrobial stewardship programs in health care systems, Clinical Microbiology Reviews, 2005, 18(4), p. 638-656.

2. When the drugs don't work, 2016, The Economist, Retrieved from: http://www.economist.com/news/leaders/216991 16-how-combat-dangerous-riseantibiotic-resistance-when-drugs-donu2019t-work.

3. Centers for Disease Control and Prevention, Core elements of hospital antibiotic stewardship programs. Atlanta, GA: CDC, US Department of Health and Human Services, 2014.

4. Centers for Disease Control and Prevention, Antibiotic resistance threats in the United States, 2013, 2013, Atlanta: CDC, US Department of Health and Human Services, 2013.

5. Van Woezik, A.F., Braakman-Jansen, L.M.A., Kulyk, O., et al., Tackling wicked problems in infection prevention and control: a guideline for co-creation with stakeholders.

Antimicrobial Resistance \& Infection Control, 2016, 5(20).

6. Dik, J.-W.H., Poelman, R., Friedrich, A.W., et al., An integrated stewardship model: Antimicrobial, infection prevention and diagnostic (AID), Future Microbiology, 2016, $11(1)$, p. 93-102.

7. Van Limburg, M., Wentzel, J., Sanderman, R., et al., Business modeling to implement an eHealth portal for infection control: a reflection on co-creation with stakeholders. JMIR Research Protocols, 2015, 4(3), p. e104.

8. Van Gemert-Pijnen, J.E., Nijland, N., Van Limburg, M., et al., A Holistic Framework to Improve the Uptake and Impact of eHealth Technologies. Journal of Medical Internet Research, 2011, 13(4), p. el11.

9. Van Gemert-Pijnen, J., O. Peters, and H.C. Ossebaard, Improving eHealth, 2013 : Eleven International Publishing Den Haag, The Netherlands.

10. Kelders, S., et al. eHealth: combining psychology, technology and health, 2016, cited 2016 08-04, Massive Open Online Course. Available from: https://www.futurelearn.com/courses/ehealth.

11. Beerlage-de Jong, N., Eikelenboom-Boskamp, A., Voss A., et al., Combining usercentered design with the persuasive systems design model; the development process of a web-based registration and monitoring system for healthcare-associated infections in nursing homes, International Journal on Advances in Life Science, 2014, 6(3\&4), p. 262 271.

12. Oinas-Kukkonen, $\mathrm{H}$. and Harjumaa, M., Persuasive systems design: Key issues, process model, and system features. Communications of the Association for Information Systems, 2009, 24(1), p. 28.

13. Beerlage-de Jong, N., Wentzel, J., Dik, J.-W., et al., Opening the black box of Clinical Decision Support Systems for Antimicrobial Stewardship - A scoping review, International Journal of Medical Informatics, Submitted.

14. Limburg, A.H.M., Implementing antibiotic stewardship: involving stakeholders in eHealth, 2016, University of Twente: Enschede, the Netherlands.

15. van Limburg, M., Sinha, B., Lo-Ten-foe, J., et al., Evaluation of early implementations of antibiotic stewardship program initiatives in nine Dutch hospitals. Antimicrobial Resistance and Infection Control, 2014, 3(33).

16. Limburg, M.V., et al. Co-creating with stakeholders: ideating eHealth applications to support antibiotic stewardship in hospitals. The 8th International Conference on eHealth, Telemedicine, and Social Medicine (eTELEMED 2016), IARIA, 2016.

17. Wentzel, J., Van Velsen, L., Van Limburg, M., et al., Participatory eHealth development to support nurses in antimicrobial stewardship. BMC medical informatics and decision making, 2014, 14(45). 
18. Wentzel, M.J., Keeping an eye on the context: participatory development of eHealth to support clinical practice, 2015: University of Twente, Enschede, the Netherlands.

19. Rosenfeld, L. and Morville, P., Information architecture for the World Wide Web: Designing large-scale web sites. 2002: O'Reilly Media, Inc.

20. Chaboksawar, T., Evaluatie van de gebruiksvriendelijkheid en persuasiviteit van een webbased applicatie Een vergelijking tussen het oude en het nieuwe systeem, in Psychology, Health \& Technology, 2014, Bachelor thesis, University of Twente: Enschede.

21. Streefkerk, R.H., Borsboom, G.J.J.M., Van Der Hoeven, C.P., et al., Evaluation of an algorithm for electronic surveillance of hospital-acquired infections yielding serial weekly point prevalence scores. Infection Control \& Hospital Epidemiology, 2014, 35(7), p. 888-890.

22. Streefkerk, R.H., Moorman, P.W., Parlevliet, G.A., et al., An automated algorithm to preselect patients to be assessed individually in point prevalence surveys for hospitalacquired infections in surgery. Infection Control \& Hospital Epidemiology, 2014, 35(7), p. 886-887.

23. Van Mourik, M.S., Troelstra, A., Van solinge, W.W., et al., Automated surveillance for healthcare-associated infections: opportunities for improvement. Clinical Infectious Diseases, 2013, 57(1), p. 85-93. 



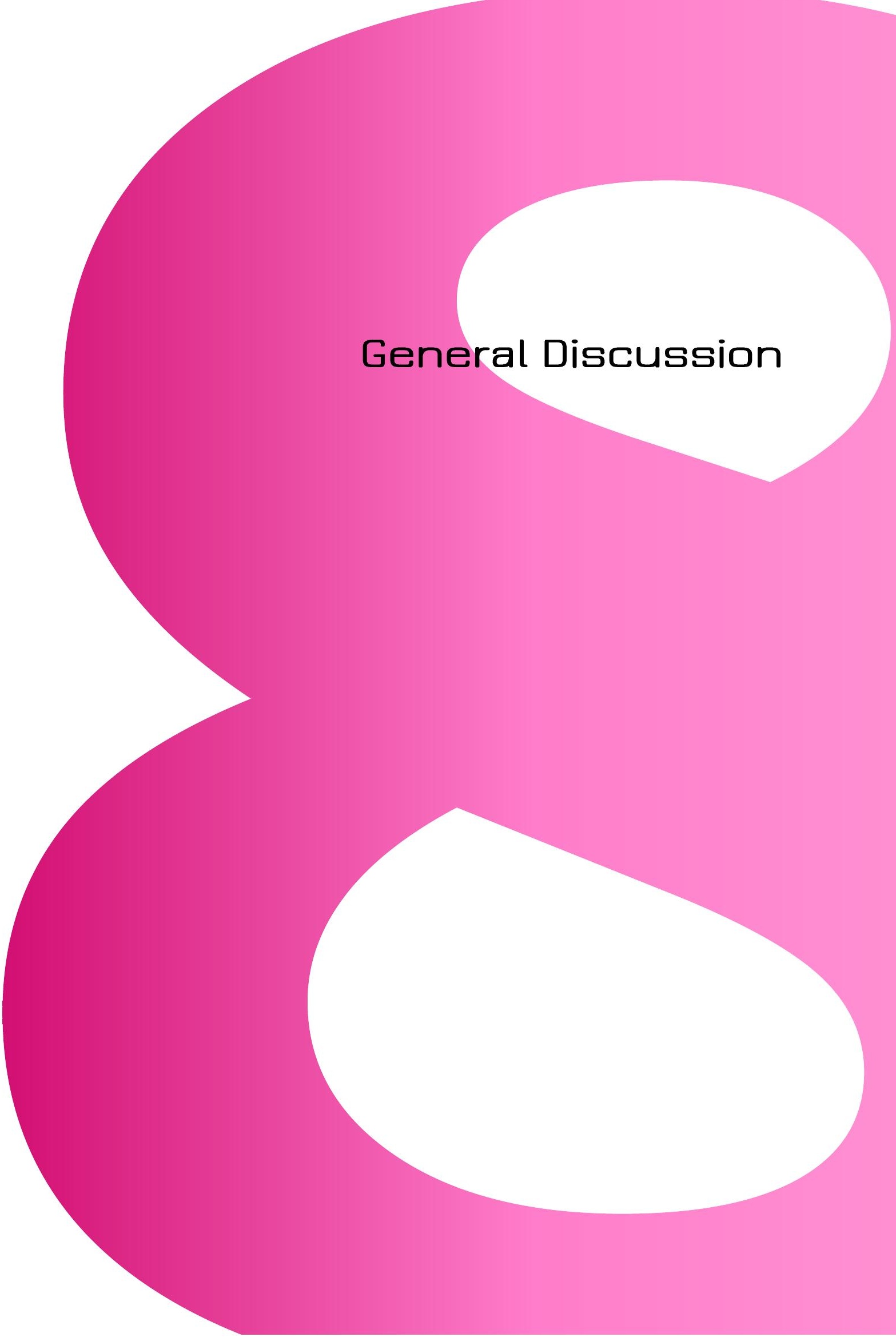




\section{Discussion}

The aim of this thesis was to investigate how eHealth technology can be best incorporated into ASPs to support healthcare professionals and stimulate safe care for their patients. Specifically, we focused on how participatory development and persuasive design can be applied for that purpose. In doing so, we aimed to contribute to both science and clinical practice. Our contribution to science consists of expanding the CeHRes roadmap with indepth knowledge about its methodologies. We contribute to clinical practice by developing eHealth technology that best fits the working and thinking processes of physicians in ASPs.

In this final chapter, the main findings and lessons learned from the different parts of this thesis are discussed. Based on these findings, the research questions that were central in this thesis are answered. Then, the strengths and limitations of the research are considered, and we (as well as this thesis) come to a conclusion.

\section{Main findings}

This thesis was divided into four parts, each focusing on a different aspect of technology: 1) participatory development, 2) persuasive design, 3) measuring persuasiveness, and 4) a reflection on our approach. In this section, we briefly describe each of these parts and their main findings.

\section{Part 1: Participatory development}

Part 1 has shown that a participatory development approach using scenario-based usertests yields valuable insights, not only into what a technology should look like and do, but also into the end-users and their working and thinking processes. Part 1 focused on participatory development, and some of its main findings were:

- Current Clinical Decision Support Systems (CCDSs) to support ASPs are expert- and policy-driven. Consequently, their supportiveness is limited, adherence suboptimal, and achieving a fit between CDSS and its use context remains challenging (Chapter 2).

- Performing prototype evaluations as part of participatory development allows potential end-users to verbalize their way of working and to think outside of their own reference framework (Chapter 3).

- Scenario-based prototype evaluations render insights into end-users' perceived needs and expectations. Cross-referencing them alongside other stakeholders can reveal unrecognized needs and provide novel into medical practice (Chapter 3).

- Such unrecognized needs and novel insights were also found for CDSSs to support ASPs. For example, end-users indicated not needing support for the selection of diagnostic tests. However, the urgency of such support was demonstrated through a 
lack of consensus (among experts and non-experts) regarding clinical practice, and the relevance of such support is subscribed in the literature [1,2] (Chapter 3).

In summary, Part 1 has taught us that involving end-users and other stakeholders during the development process of eHealth technology for ASPs provides insights into the needs and expectations that the technology has to meet as well as the (implicit) work practices in clinical care that prescribe its context.

\section{Part 2: Persuasive design}

Combining a participatory development approach with elements of the Persuasive Systems Design (PSD) model [3] increases the chances of sustained use of and adherence to the technology [4]. Part 2 of this thesis demonstrated how this combination can be achieved and the value of doing so by describing the development of a web-based registration and monitoring system for Healthcare-Associated Infections (HAls) in nursing homes. Part 2 evolved around the combination of participatory development and persuasive design in eHealth technology and taught us:

- Evaluating current clinical practice (via the PSD model) is a crucial first step to determining what elements of persuasion to apply in what situation.

- A clickable prototype of a registration system was developed based on contextual inquiry. The prototype incorporated elements of persuasion that were relevant for and capable of dealing with the determined prerequisites, needs and values. For example, registering clients was very laborious, requiring elderly care physicians to read and click through many redundant questions. Tunneling allowed us to omit questions that were redundant for the specific client based on previous answers, making registration much more efficient. Scenario-based user-tests were performed, to validate the decisions that were made about which persuasive elements to incorporate and how (Chapter 4).

- By applying persuasive design, physicians can be supported in performing their tasks faster, easier and with less chance of error (Chapter 4) [5].

In all, Part 2 demonstrates how participatory development can be combined with persuasive design and shows that the persuasive elements of a technology can and should be adapted to its specific end-users, context and aims. This adaptation can be achieved by thoroughly studying where problems are located and by incorporating persuasive elements during the early stages of the participatory development.

\section{Part 3: Measuring persuasiveness}

Besides the development of technology, its evaluation is also crucial to ensuring it remains usable [6]. Performing such evaluations in the field of eHealth technology is far from easy. Its success depends on a multitude of factors, such as persuasiveness, usability and reliability, each of which can contribute to eHealth success in its own way. The persuasive influence on users may also be impacted by whether end-users actually notice the 
persuasive elements. Part 3 focused on evaluating persuasiveness. It showed the relevance and potential of the Perceived Persuasiveness Questionnaire (PPQ) [7] as a tool to evaluate to what extent technology users experience its persuasive elements. Our findings showed:

- Results that are found via the PPQ are consistent with results that are found through other methods (expert evaluation and user-tests), indicating that it is a promising tool to evaluate perceived persuasiveness (Chapter 5).

- A card sort and user evaluation of an mHealth app indicated that whereas most PPQ constructs reached adequate construct validity (i.e., Perceived Dialogue Support, Perceived Social Support, Perceived Effort, Perceived Credibility, Use Continuance, Perceived Unobtrusiveness), some constructs should be adjusted. Cluster analysis revealed that Perceived Primary Task Support, Perceived Persuasiveness and Perceived Effectiveness actually represented a single cluster, perhaps best described as Perceived Goal Support (Chapter 6).

On the whole, we learned that the PPQ offers a very promising way to evaluate perceived persuasiveness. However, it also requires some additional work for it to reach its full potential because its construct validity can be improved, and the questionnaire should still be validated.

\section{Part 4: A reflection on our approach}

Finally, in Part 4 we looked back on some of our projects in which we applied the CeHRes Roadmap [8] and what lessons these projects taught us. Consequently, we expanded on the CDC report on core elements of ASPs [2] by elaborating on how technology can be developed and implemented to support these core elements. We found that:

- The CeHRes Roadmap provides a framework to guide the participatory development and implementation of a technology-supported integrated stewardship program by offering methods for the involvement of end-users (for prototype evaluations) and other stakeholders (for determining conditions for implementation).

To conclude, in Part 4, we reflected on our approach to eHealth development, offered practical guidance on how to apply the CeHRes Roadmap, and described what kind of knowledge that might possibly yield.

\section{Implications for participatory eHealth development for ASPs}

The main findings that are described in this thesis have consequences for eHealth technology for ASPs. In this section, we discuss the implications that our findings have for the participatory development of such technologies. Following that, we explore the implications for such technologies' persuasive design.

ASPs have a strong medical foundation and clinical focus [9]. This appears inherent, due to the very nature of such programs, but, at the same time, we have shown that such a 
strong medical and clinical focus might be the ASPs' most troublesome characteristic. A strong clinical focus implies a lack of attention to the contextual, social or behavioral aspects of ASPs. Consequently, the instructions and/or guidelines that are used for the execution of ASPs do not often fit the user or the use context, leading to suboptimal use of and adherence to ASP guidelines, which in turn results in suboptimal clinical effects. This consequence is not only found in prior research on the use of technology in ASPs [10-12], but is also confirmed by our own research [9]. Over the last decades, several studies have demonstrated a need for a more social or behavioral approach to ASPs [13, 14]. Confirmation is further demonstrated in the most recent report of the Centers for Disease Control and Prevention. In this report on hospital ASPs, numerous recommendations have been called for, such as leadership commitment, the appointment of an ASP physicianleader, and the feeding back of information about process measures to clinical staff [2]. In light of these findings and recommendations, we described a behavioral approach to developing eHealth technology for ASPs and then examined its added value.

The first sub-question of this thesis was: How can a participatory development process of eHealth technology contribute to supporting physicians in ASPs? Every ASP starts with the common aim to protect patient safety by limiting the occurrence of antimicrobial resistance along with ideas about what should be changed in the clinical practice to achieve this goal. However, the end-users (such as, the physicians and nurses) and the context (that is the focus, clinical setting, and stakeholders) of every ASP differ. Even the context of the interventions within a single ASP may differ from one another. Dealing with such diverse conditions leads to different prerequisites for ASPs. These prerequisites for an ASP are its basic foundation and consist of (among others) the needs of the end-users (e.g., physicians and nurses), their wishes and expectations, and the context in which the ASP is used. For example, earlier studies provided insights into the problems with infection control guideline adherence $[11,15]$. They showed that such guidelines must unite two functions that are almost impossible to unite - a 'regulation function' (i.e., rules and legislation) and a 'communication function' (i.e., offering a means of communication for individual healthcare providers) $[11,15]$. Without paying attention to such diverse needs and expectations, an ASP may be clinically relevant, but not clinically practical. Participatory development can reveal and deal with such differing needs and expectations.

Without adequate attention to an ASP's development process and without taking the right steps during such development, physicians and others who must use the ASP might not be willing or able to work with it, causing the ASP to not succeed. However, Chapter 2 has shown that currently, the development process of eHealth technology for ASPs is often overlooked [9]. Often no information at all is provided about the development of (in this case) CDSSs for ASPs. When information is provided, the systems are frequently based on a single type of input data provided by experts or based on guidelines, and consequently support for the technology that is used in the ASP remains limited. Elsewhere, other researchers have already suggested that low eHealth technology use and adherence rates were related to (a lack of) end-user involvement $[16,17]$. To overcome this problem, we demonstrated that end-users and other stakeholders should be involved in the 
(participatory) development process of the technology in order to increase its userfriendliness and engage the end-users' support. This is especially true since the topic of ASPs and the behavioral changes that they require are a 'wicked' problem, which means that it will not and cannot be solved by a single solution [18]. Moreover, such problems require close cooperation with stakeholders to be able to deal with them [18]. Chapter 3 has shown what a participatory development process may look like and its possible added value.

Based on our research, we propose a number of prerequisites for successful participatory development of eHealth to support ASPs as described in the following sections.

\subsection{Involve end-users from the development through the evaluation of technology}

Especially in healthcare, which is a highly complex work environment, it is not advisable to rely solely on experts to determine how ASPs can be designed to fit its end-users. Rather, why not ask the end-users themselves? The research in this thesis has shown that such an approach is paramount (Chapter 2 \& 3). But perhaps more importantly, we have demonstrated that, as long as end-users are given the opportunity and some support, they are very capable of visualizing and verbalizing their needs and expectations (Chapter 3 ). One way to aid them in this process is through the presentation of real-life scenarios and prototypes of an eHealth technology, which help stimulate the verbalization of end-users' own processes at work and promote their out-of-the-box thinking about the possibilities of working with the technology (Chapter 3).

The positive effects of such an approach are evident even with low-fidelity paper-based sketches of what a technology might look like, especially when combined with real-life scenarios. Applying such prototypes in Chapter 3 revealed gaps in the ASP's support of physicians who must prescribe antimicrobial therapies in their current clinical practice. For example, the prototype offered advice for the selection of an appropriate antimicrobial treatment with a software screen in the prototype displaying the kind of information that could be provided. This caused participants to realize and verbalize that they found it difficult (or even impossible) to find information about the appropriate duration of an antimicrobial therapy in the current sources. They indicated that the duration was often determined more or less 'at random'. This is a worrisome finding, as the duration of an antimicrobial therapy is a relevant aspect of ASPs. Fortunately, participants indicated that the technology that was proposed in the prototype was very useful in supporting them in selecting an appropriate duration.

\subsection{Involve multiple stakeholders from the development through the evaluation of technology}

Involving end-users in the development of eHealth technology to support ASPs provided us with a clear idea about what they wanted and needed. However, as Elsschot wrote more than a century ago: "In between dream and act, there are hindering laws and practical 
objections" [19]. Throughout the development of an ASP, practical objections and laws require the involvement of multiple stakeholders (i.e., experts in all kinds of fields) to crossreference the needs, wishes, and expectations of potential end-users and to make sure that their needs are translated into achievable and appropriate interventions. In their recent report on Antimicrobial Stewardship, the Centers for Disease Control and Prevention also emphasized the importance of involving various stakeholders in establishing ASPs in hospitals [2]. Some of the stakeholders that might be involved include: hospital administration for financial support as well as the legitimacy of the ASPs, experts in the field of medical microbiology for their indispensable knowledge about the required content of ASP interventions, and experts in the field of ICT for their understanding of the possibilities and limitations of the technology and of how to integrate it with any existing systems.

Chapter 3 demonstrated how such involvement can be realized and what kind of knowledge it yields. Within the research described in this chapter, end-users were able to clearly state their needs, wishes and expectations of the studied eHealth technology to support ASPs. They also indicated what kind of support they did not need. Specifically, end-users indicated they did not need a CDSS to support them in selecting appropriate diagnostic tests, which is a requirement for prescribing appropriate antimicrobial therapy. This opinion was then cross-referenced among a larger group of physicians and experts in the used case, revealing that, although end-users were consulted, some unrecognized needs could still and did exist. We found that hardly any consensus existed about what diagnostic tests to perform, both among prescribing physicians and among (lung) infection experts. From a scientific perspective, this finding clearly calls for the involvement of multiple stakeholders in the development of eHealth technology to support physicians in ASPs. In addition, from a clinical point of view, this implies that (persuasive) strategies have to be applied to pro-actively support physicians in selecting appropriate diagnostic tests.

\subsection{Keep a clear view on protocols and guidelines}

As briefly mentioned in Section 2.2, besides the needs, wishes and expectations of endusers, ASPs must also adhere to certain rules and regulations, initiated to ensure safety and the application of evidence-based medicine. If such rules and guidelines are not accounted for, an ASP can be clinically relevant but ultimately insufficient or even terminated because it does not fit the context in which it must be applied.

From the start of any ASP, its cornerstone is the protocols and guidelines that reflect the regulations and state-of-the-art knowledge about antimicrobial therapy. Consequently, in this thesis, protocols and guidelines appear as the cornerstone for the prototypes of eHealth technology (Chapters $3 \&$ \&). While it remains important to develop and implement user-friendly and usable eHealth technology to support ASPs, its content still must be based on medical protocols and guidelines. If the protocols and guidelines are neglected, it becomes impossible for physicians to trust and work with the system. Prior 
research has demonstrated that for existing protocols and guidelines to be applicable in practice, they must be "translated" to fit clinical practice [11, 12].

We further demonstrated that both procedural and declarative information are required and should be provided in protocols and guidelines (whether communicated through eHealth technology or not)[20]. Procedural information (i.e., information about the tasks to be performed) should be available at any time, while declarative information (i.e., information about why tasks should be performed) should be available and easily accessible upon request (see also Chapter 3). Chapters 3 and 4 have shown that eHealth technology can greatly contribute to communicating both procedural and declarative information in addition to aiding to differentiate between the two [21]. Chapters 3 and 4 demonstrated the need for point-of-care procedural information, such as steps to be taken to prudently prescribe antimicrobials. Chapter 3 has shown that physicians also needed easy access to additional in-depth (background) information and to information about the reasoning behind why a specific antimicrobial was suggested. Thus, an interesting challenge for eHealth technology in ASPs lies in alerting physicians in a timely fashion that they need additional information, but also in providing it to them in usable and relevant chunks.

Our research revealed some challenges for protocols and guidelines as well. Especially for physicians, their 'medical autonomy' allows them to customize the available medical standards for each individual patient, keeping in mind the patient's unique characteristics, medical history and other relevant circumstances (see also Chapter 3 ). Therefore, while protocols and guidelines should account for such medical autonomy by allowing physicians some degree of freedom, the same goes for successful eHealth technology as well. For example, for technologies such as CDSSs for ASPs, many factors play a role in physicians prescribing the optimal antimicrobial therapy. In such cases, technology should be used to guide and structure thought processes and to support physicians in thinking beyond the borders of their medical specialty [9, 22]. Potential end-users of CDSSs indicated that for them to be willing to work with the technology, it should always leave them some degree of freedom to account for their medical autonomy (Chapter 3 and 7). CDSSs should not and cannot be too directive since circumstances may require physicians to sometimes stray from the clinical paths that are described in the guidelines (see also Chapters 2 and 3). On the other hand, technologies such as the Prevalence app require physicians to closely follow guidelines without taking any liberties since that would harm the reliability of the prevalence measurements. This means that such technologies should be very directive and leave no room for interpretations by its end-users (see also Chapter 4).

\section{Implications for persuasive design in ASPs}

Besides all the planning and actions that are involved in the development processes of technology to support ASPs, the technologies themselves are pivotal for their success. Therefore, the second sub-question of this thesis was: How can persuasive design of 
eHealth technology contribute to supporting physicians in ASPs? One of the major threats to their (and ASPs') success are the physicians low use of and adherence rates to (eHealth technologies in) ASPs. A well-known and promising way to deal with this challenge is to apply persuasion in the eHealth technologies [23].

Specifically, we have drawn from the Persuasive Systems Design (PSD) model [3] to enrich eHealth technologies with persuasive elements. Doing so has consequences for both the development (see Section 3.1) and the evaluation (see Section 3.2) of eHealth technology.

\subsection{Developing persuasive technology}

Technologies that are developed to support physicians in ASPs aim at supporting or changing physicians' behavior. Such technologies are known as 'persuasive technology'. The PSD model offers a framework for the development of persuasive technologies. Based on our research, we have complemented the model with a more practical guideline for how the framework can be applied in practice, how it can be combined with a participatory development process, and what the added value thereof is.

In our research, we demonstrated that persuasive strategies can and should be selected based on an analysis of the context within which the technology is to be used (see Chapter 4). Based on the selected persuasive strategies, technical solutions can be sought. We have shown that the persuasive elements that are deemed relevant can and should already be incorporated during an early stage of technology development in order to check the elements' appropriateness. They can already be made visible in low-fidelity prototypes, as was shown in Chapter 4. Doing so allows for researchers and IT developers to ensure a fit between persuasive elements and technology users and the context. This early approach is of importance, since different people may in different settings, be susceptible to (or best supported through) different forms of persuasion. Scenario-based user-tests can provide insights into what works best for a specific technology. Within our research, different elements of the PSD model were applied, as follows:

- Primary Task Support is what makes it easier for end-users to work with a technology and achieve their goals (e.g., registering and monitoring Healthcare-Associated Infections). In the Prevalence app, Primary Task Support allowed us to simplify the tasks by shortening the questions, and to make each task less time consuming by omitting irrelevant questions (see Chapter 4).

- Dialogue Support is how the technology and its users interact with one another. Within the Prevalence app, we used Dialogue Support to make the system less likely to enter errors (e.g., by avoiding typing errors when inputting a complicated antimicrobial name, such as "Phenoxymethylpenicillin"; see Chapter 4).

- System Credibility Support concerns how reliable a system looks and feels, based on the end-users' first impressions or on the (authoritative) references and sources upon which it is based. The participants who tested the CDSS prototype indicated that System Credibility Support should entail administration to endorse the system (see Chapter 3). 
- Offering Social Support allows physicians to interact with and learn from peers and experts. For example, the Prevalence app offered insights into the infection rates at the physician's institution as compared to a benchmark of other participating nursing homes (see Chapter 4).

In general, we found that all these kinds of persuasive support elements are pivotal for technologies in ASPs and that they sometimes serve different purposes in different situations. For example, in the Prevalence app (aimed at monitoring infections) Dialogue Support is mainly used to support end-users in avoiding (e.g., typing) errors. This support can also be so for CDSSs (aimed at prescribing antimicrobials), but in such technologies Dialogue Support is mainly appreciated as a means to create a fit between a technology and clinical practice, by providing point-of-care reminders and alerts for using the system or performing certain tasks. Social Support was used in the Prevalence app to enable benchmark comparisons of infection (and risk factor) rates between participating nursing homes. This support can also be applied similarly in CDSSs (e.g., for audit and feedback), but then Social Support is mainly used to enable easy and fast communication between prescribing physicians and ASP experts (e.g., pharmacy and microbiology experts).

\subsection{Evaluating persuasive technology}

An important aspect of the CeHRes Roadmap concerns the ongoing formative evaluation of eHealth technology [6]. Evaluation is not only relevant for the technology as a whole, but also for its persuasiveness in particular. Therefore, the third research question of this thesis was: How can the success of persuasive eHealth technology be evaluated?

As eHealth technology is a very broad term [24], so is its success. Measuring the success of eHealth technology depends on its aims, users and context. Subsequently, the same is true for eHealth technology that is used within ASPs. Although technologies for ASPs have some prerequisites for success in common (e.g., reliability, speed), clear distinctions remain. For example, success for the Prevalence app [21] requires the technology to leave absolutely no 'wiggle room,' meaning no room for end-user interpretations. On the other hand, the CDSSs also studied in this thesis $[9,22]$ require some 'wiggle room' to be successful. Therefore, each technology requires different measures to evaluate its success, such as supporting thought processes about choices to be made and/or easy access to background information. Besides technology-specific keys for success, all technologies share the requirement that they support and motivate their users (Chapter 7). The support and motivation of end-users that a technology provides is also known as 'persuasion' [3]. To date, the influence that the persuasiveness of eHealth technology has on its success has hardly been evaluated. Yet such an evaluation is a pivotal part of opening the 'black box' of eHealth [23]. It provides insights into the working mechanisms that determine the users' experience while working with the technology and that contribute to the technology's sustained use.

Our research has shown that the Perceived Persuasiveness Questionnaire (PPQ) offers a promising way to evaluate the persuasiveness of eHealth technology in a relatively 
undemanding way (Chapters 5 and 6). Results that were rendered through the PPQ were in line with results that were found via alternative methods, i.e., expert evaluations and usability testing (Chapter 5). This finding indicates that, while the PPQ is a promising tool for measuring perceived persuasiveness, some room for improvement remains. In-depth analysis of PPQ constructs and items via a card sort analysis revealed its internal consistency and construct validity (Chapter 6). This is especially useful in the explorative stage of questionnaire evaluation, when it is not always possible to include enough participants for a factor analysis. Based on a cluster analysis with the data, we have suggested some changes in the PPQ. The constructs Perceived Dialogue Support, Perceived Credibility, Unobtrusiveness, Use Continuance, Perceived Effort and Perceived Social Support should remain more or less unchanged. On the other hand, participants struggled with sorting the items of the following constructs: Perceived Primary Task Support, Perceived Effectiveness and Perceived Persuasiveness. According to cluster analysis, these constructs should be merged into a single construct, as all the items of these three constructs revolve around how well a technology supports its users in reaching their goals. Consequently, we suggest labeling this single, merged construct as Perceived Goal Support. These changes also increase the internal consistency of the constructs.

Additionally, during the PPQ analysis, we found that its constructs do not measure the same kind of entities. For example, some are outcome-oriented, such as Perceived Effectiveness while others are for example usability-oriented, such as Perceived Dialogue Support. It remains to be seen and requires further analysis to determine whether it is desirable to measure such various entities in a single questionnaire. For example, perhaps persuasiveness inherently entails such differing entities, which also appears more or less true for the items within each construct. Currently, the items measure different aspects from the scope of a construct. However, it is almost impossible to cover the entire range of a construct, especially for a generic questionnaire that must be applicable for various technologies. For example, a technology that aims at encouraging healthy sleep patterns may require a different kind of Dialogue Support as compared to a CDSS for ASPs. While the former would not provide too much information (after all you want to go to sleep!), the latter would require adequate information to enable evidence-based medicine. Nevertheless, all technologies do benefit from some form of Dialogue Support. Thus based on the findings of Chapter 6, we suggest adapting the PPQ to measure each PSD construct through two or three very similar items. By doing so, a PPQ item can inquire about a construct as a whole instead of only a small aspect of it. A secondary benefit of this approach is that the PPQ might decrease in length, making it even less demanding for the respondents.

\section{Strengths and limitations}

Our research has several strengths and limitations. The field of Antimicrobial Stewardship still is a relatively new field, providing many possibilities to be explored. This thesis bundles several studies within a single research project, enabling us to investigate and study the challenges surrounding ASPs from several perspectives and within several contexts. Such 
a comprehensive project enabled us to check whether our assumptions about the added value of participatory development and persuasive design held true for multiple settings. However, one limitation of this method is that it did prevent us from executing a single project that follows the CeHRes Roadmap from start until "finish," even though we do not eHealth technology is ever finished [6].

Consistent with the explorative nature of this research project, longitudinal analysis of the effects of the proposed development and design of eHealth technology was not performed. While this might be viewed as a limitation, in the overall field of ASPs, such analyses are scarce. Effects on the eventual aim of ASPs, such as diminishing antimicrobial resistance, are especially under evaluated, due to the fact that measuring such aims is highly complex and requires long-term measurements [25]. Future research should definitely study the effects of applying eHealth technology to aid in reaching such goals.

Moreover, it would be worth studying the effects of applying a participatory development approach. In our research, we have shown that involving multiple stakeholders can yield more information than cooperating with end-users only. Different stakeholders offer diverse perspectives regarding work processes and how eHealth technology can support them. However, we did not compare the effects of participatory development with, for example, user-centered development (wherein only end-users are involved). However, prior research has suggested that the 'mere' participation of stakeholders during its development may already have a positive effect on their use and acceptance of the technology [17]. This finding makes a participatory development process (via the CeHRes Roadmap) a pivotal part of the implementation of the eventual technology [6]. Researching such compelling questions requires a close cooperation between science (for planning of and reporting on the study), healthcare (as a research setting) and business (for processing data). Such cooperation is currently shaping the successor of the EurSafety Health-net project, namely the INTERREG Va Health-i-Care project.

As previously mentioned, in addition to incorporating the entire process of the CeHRes Roadmap into the development of eHealth technology for ASPs, protocols and guidelines remain the cornerstone of ASPs. Unfortunately, within the scope of this thesis, we were unable to extensively focus on the development of the protocols and guidelines themselves. However, from prior research, we already realize that much remains to be accomplished by research in that field as well $[26,27]$. First of all, we already mentioned that the fit between protocols and guidelines with clinical practice is paramount and requires more attention to reach their full potential $[11,12]$. To ensure such a fit, end-users should be involved in the development of such protocols. Moreover, we believe that protocols and guidelines should not only contain clear information about what tasks should be performed and why, but also about who is responsible for performing those tasks. This additional information, in combination with involving end-users in their development, can contribute to creating more 'problem ownership' among healthcare professionals, which increases their awareness of the need for changing their own behavior. 
Another strength of our research is that it uses mixed-methods to answer each of its research questions. This allowed us to immediately cross-reference our findings, leading to more robust results. Although the focal points of this thesis were thus examined from multiple perspectives, we also omitted some aspects of the CeHRes Roadmap. For our research, the CeHRes Roadmap provided a framework for a structured focus on and integration of participatory development and persuasive design. As a consequence of this focus, performing business modeling received less attention than might have been desired according to the Roadmap. However, the business part of ASPs was more elaborately studied in an adjacent research project [10]. The chosen focus for the research in this thesis may have contributed to some of the challenges that persisted for the sustainability (e.g., maintenance) of the Prevalence app. The sustainability challenges, however, correspond with those found in prior research of eHealth technology in ASPs [28]. Such barriers may be illustrative of our assumption that "eHealth is never finished" [6], since it can always be improved and should continuously be adapted to changing circumstances.

\section{Implications for future research and practice}

As we have stated, this thesis incorporated two main pillars of the CeHRes Roadmap participatory development and persuasive design - allowing us to provide in-depth knowledge about some aspects of the Roadmap (e.g., how can participatory development be approached). Our chosen focus automatically meant that a third pillar (i.e., business modeling) was omitted in our research. This also goes for some of the stages of the Roadmap itself, such as the implementation stage. It would be interesting for the Roadmap to reach its full potential by applying it as a whole for the development of an eHealth technology to support ASPs. This would result in a well-grounded, tested, and evaluated sustainable technology to successfully support ASPs while simultaneously allowing researchers to thoroughly test and discover the strengths and limitations of the CeHRes Roadmap.

We also strongly believe that our approach is of great added value for supporting the sustained use of and adherence to eHealth technology as suggested by prior studies [4, 10-12]. Nevertheless, the long-term effects of our approach towards eHealth development have yet to be evaluated in a longitudinal study. It would be very interesting to further explore this phenomenon. For example, future research might compare commitment and adherence to top-down developed expert- or guideline-driven eHealth technology with that of eHealth technology that was developed following the Roadmap. Additionally, it would be interesting to explore (e.g., via MOST design [29, 30]) the actual added value of applying persuasive design. In this case, it would be worthwhile to differentiate between three kinds of eHealth technology: (1) technology that does not explicitly make use of PSD; (2) technology that was developed by experts, who also determined what persuasive elements to use; and (3) technology incorporating elements of persuasion that were deemed relevant according to stakeholders. Besides revealing the effects of persuasion, 
such a study would also provide insights into "what works, when, and for whom," when it comes to persuasion.

In addition, more remains to be explored about persuasion, specifically, the way it can be evaluated through the PPQ. Our research has demonstrated that the development and validation of such a questionnaire is very much needed and worthwhile [31]. Our work also showed that some additional research is required, and follow-up steps are suggested. Fortunately, the end of this Ph.D. project by no means ends this research project. Many of the opportunities that were uncovered in this thesis are currently being applied and developed further in projects that are closely allied to the current one. For example, a reevaluation is being conducted on how to best represent the PSD model [23] in a PPQ [32] and how a PPQ can account for the differences in their frame of reference. Of course, after taking a step back and reconsidering the PPQ, future research should also perform a factor analysis to adequately validate the questionnaire.

\section{To conclude}

Let us recall the very first question of this thesis. Remember that wise scientist sitting behind his desk, pondering how he might make the world a safer place for patients? Based on what we have learned in this research project, we have sadly come to doubt his wisdom. Unless, of course, he is sitting there thinking about the best or most innovative way to include stakeholders in the development of eHealth technology to support physicians in ASPs.

This thesis has shown that, although it is far from having reached its full potential, persuasive technology holds great promise for supporting healthcare professionals in ASPs. To ensure that a technology is as supportive as it can be, end-users can and should be involved in its development so that it might better fit their needs, wishes and expectations. Merely asking about these needs, wishes and expectations does not suffice, as visualizing and verbalizing their way of working and what it would be like to work with technology is close to impossible for most end-users. Instead, scenario-based prototype evaluations, even low-fidelity (paper-based) prototypes, can provide valuable insights for eHealth technology as end-users think about their work processes and the possible opportunities for technological support. To make sure their needs, wishes and expectations are reconcilable with their clinical practice, other stakeholders (e.g., hospital administration, IT specialists and clinical microbiologists) must continuously be involved to cross-reference the findings with practical application. Finally, protocols and guidelines must always be kept in mind, as they are the cornerstone of the content that should be communicated through any technology.

Applying persuasion in ASP technology is a valuable means of surmounting the relatively low use and adherence rates by end-users that limit the success of the ASPs' technology. However, the kind of persuasion should be tailored to the technology's users and the context. Finding the best fit between persuasion, user, and context can also be achieved via scenario-based prototype evaluations. This thesis has demonstrated how different kinds 
of persuasion can be successfully applied and evaluated in low-fidelity prototypes. For the evaluation of the effects of persuasion, the PPQ offers a promising means to study the effects without requiring much effort from participants.

For clinical practice, this thesis has confirmed our strong belief that ASPs require a 'network' of technologies to support the entire care process. This network of technologies ranges from supporting diagnostics, prescribing antimicrobials, administrating antimicrobial therapy, and applying infection control measures. It is important to note that all these technologies must support people working in a highly complex healthcare environment, with ever changing conditions. This requires that each technology and their entire network provide the necessary information or decision support without undermining or disabling medical autonomy.

In summary, the knowledge and insights described in this thesis were gained from closely cooperating with end-users and other stakeholders in ASPs. As one final thought, may I suggest that the next time you think about the wise scientist who wants to make the world a safer place, you picture a young, brown-haired female who has a desk, but merely uses it to analyze what she has learned in practice! 


\section{References}

1. Dik, J.-W.H., Poelman, R., Friedrich, A.W., et al., An integrated stewardship model: Antimicrobial, infection prevention and diagnostic (AID), Future Microbiology, 2016, $11(1)$, p. 93-102.

2. Centers for Disease Control and Prevention, Core elements of hospital antibiotic stewardship programs. Atlanta, GA: CDC, US Department of Health and Human Services, 2014.

3. Oinas-Kukkonen, H. and Harjumaa, M., Persuasive systems design: Key issues, process model, and system features. Communications of the Association for Information Systems, 2009, 24(1), p. 28.

4. Kelders, S.M., Understanding adherence to web-based interventions, 2012, Universiteit Twente: Enschede, the Netherlands.

5. Chaboksawar, T., Evaluatie van de gebruiksvriendelijkheid en persuasiviteit van een webbased applicatie Een vergelijking tussen het oude en het nieuwe systeem, in Psychology, Health \& Technology, 2014, Bachelor thesis, University of Twente: Enschede.

6. Van Gemert-Pijnen, J.E., Niiland, N., Van Limburg, M., et al., A Holistic Framework to Improve the Uptake and Impact of eHealth Technologies. Journal of Medical Internet Research, 2011, 13(4), p. e111.

7. Lehto, T., Oinas-Kukkonen, H., and Drozd, F., Factors Affecting Perceived Persuasiveness of a Behavior Change Support System. In: '33th International Conference on Information Systems, 2012, Association for Information Systems, Orlando, Florida.

8. Beerlage-de Jong, N., Van Gemert-Pijnen, J.E.W.C., Wentzel, J., et al., Technology to support integrated Animicrobial Stewardship Programs; A user centered and stakeholder driven development approach. Infectious Disease Reports, Submitted.

9. Beerlage-de Jong, N., Wentzel, J., Dik, J.-W., et al., Opening the black box of Clinical Decision Support Systems for Antimicrobial Stewardship - A scoping review, International Journal of Medical Informatics, Submitted.

10. Limburg, A.H.M., Implementing antibiotic stewardship: involving stakeholders in eHealth, 2016, University of Twente: Enschede, the Netherlands.

11. Verhoeven, F., When staff handle staph: user-driven versus expert-driven communication of infection control guidelines, 2009, University of Twente: Enschede, the Netherlands.

12. Wentzel, M.J., Keeping an eye on the context: participatory development of eHealth to support clinical practice, 2015, Universiteit Twente: Enschede, the Netherlands.

13. Hulscher, M.E., Grol, R.P., and Van Der Meer J.W., Antibiotic prescribing in hospitals: a social and behavioural scientific approach. The Lancet Infectious Diseases, 2010, 10(3), p. 167-175.

14. Janes, C.R., Corbett, K.K., Jones, J.H., et al., Emerging infectious diseases: the role of social sciences. The Lancet, 2012, 380(9857), p. 1884-1886.

15. Gemert-Pijnen, J., Het totstandkomen en functioneren van infectiepreventieprotocollen. Een onderzoek naar communicatie gestuurd door wet-en regelgeving, 2003, University of Twente: Enschede, the Netherlands.

16. Hum, R.S., Cato, K., Sheehan, B., et al., Developing clinical decision support within a commercial electronic health record system to improve antimicrobial prescribing in the neonatal ICU. Applied Clinical Informatics, 2014, 5(2), p. 368-387.

17. Nachtigall, I., Tafelski, S., Deja, M., et al., Long-term effect of computer-assisted decision support for antibiotic treatment in critically ill patients: A prospective 'before/after' cohort study, BMJ Open, 2014, 4(12), p. e005370.

18. Van Woezik, A.F., Braakman-Jansen, L.M.A., Kulyk, O., et al., Tackling wicked problems in infection prevention and control: a guideline for co-creation with stakeholders.

Antimicrobial Resistance \& Infection Control, 2016, 5(20).

19. Elsschot, W., The marriage (het huwelijk), 1910. 
20. Karreman, J., Ummelen, N., and Steehouder, M., Procedural and declarative information in user instructions: What we do and don't know about these information types. In: Proceedings of the International Professional Communication Conference, 2005, IEEE International, 2005, p. 328-333.

21. Beerlage-de Jong, N., Eikelenboom-Boskamp, A., Voss, A., et al., Combining usercentered design with the persuasive systems design model; the development process of a web-based registration and monitoring system for healthcare-associated infections in nursing homes. International journal on advances in life sciences, 2014. 6, p. 262-271.

22. Beerlage-de Jong, N., Wentzel, M.J., Hendrix, R., et al., The value of Participatory Development to support Antimicrobial Stewardship with a Clinical Decision Support system. American Journal of Infection Control, In review.

23. Kelders, S.M., Kok, R.N., Ossebaard, H.C., et al., Persuasive system design does matter: a systematic review of adherence to web-based interventions. Journal of medical Internet research, 2012. 14(6), p. e152.

24. Eysenbach, G., What is e-health? Journal of medical Internet research, 2001. 3(2), p. e20.

25. Dik, J.-W., Hendrix, R., Poelman, R., et al., Measuring the impact of Antimicrobial Stewardship Programs. Expert Review of Anti-infective Therapy, 2016.

26. Jong, N. and Gemert-Piinen, J.E.W.C., Gebruikersgerichte basishygiëneprotocollen: de toekomst voor veilige zorg. Infectieziekten bulletin, 2013. 8(24), p. 258-262.

27. Jong, N., Intervention Mapping:: bevorderen van de naleving van basishygiëneprotocollen door medewerkers van Medisch Spectrum Twente, 2010, University of Twente.

28. Wentzel, M. and Nijdam, L., Understanding eHealth use from a Persuasive System Design perspective: an Antibiotic Information Application for Nurses. International journal on advances in life sciences, 2014. 6, p. 210-219.

29. Collins, L.M., Murphy, S.A., and Strecher, V., The Multiphase Optimization Strategy (MOST) and the Sequential Multiple Assignment Randomized Trial (SMART): New Methods for More Potent eHealth Interventions. American journal of preventive medicine, 2007. 32(5 Suppl), p. S112-S118.

30. Collins, L.M., Murphy, S.A., Nair, V.N., et al., A strategy for optimizing and evaluating behavioral interventions. Annals of Behavioral Medicine, 2005. 30(1), p. 65-73.

31. Beerlage-de Jong, N., Kulyk, O.A., Kuonanoja, L., et al., Evaluation of the Perceived Persuasiveness Questionnaire. International Journal of Human-Computer Studies, Submitted.

32. Lehto, T., Oinas-Kukkonen, H., and Drozd, F., Factors Affecting Perceived Persuasiveness of a Behavior Change Support System. in In: 'Thirty Third International Conference on Information Systems (ICIS2012). 2012. Orlando, Florida, USA, Association for Information Systems. 



\section{Samenvatting}

[Summary in Dutch] 


\section{Samenvatting [Summary in Dutch]}

In Hoofdstuk 1 is één van de grootste bedreigingen van de gezondheid(szorg) beschreven: antimicrobiële resistentie. Elke keer wanneer iemand een antimicrobieel middel (zoals een antibioticum) gebruikt, worden de ziekteverwekkers (bacterie, virus, schimmel) waartegen het middel gebruikt wordt er een beetje meer resistent tegen. Als dit proces niet vertraagd of gestopt wordt kunnen alledaagse aandoeningen die nu eenvoudig te behandelen zijn, zeer ernstig of zelfs fataal worden. Het is daarom van groot belang dat antimicrobiële middelen op de juiste manier worden voorgeschreven.

Door een toename in resistente ziekteverwekkers neemt ook het risico op zorggerelateerde infecties toe. Dit zijn infecties met een resistente ziekteverwekker, die opgelopen kunnen worden tijdens een opname in een zorginstelling. Behalve dat dergelijke infecties moeilijk te behandelen zijn, verspreiden ze zich ook razendsnel onder zowel patiënten als zorgverleners.

Om dergelijke processen tegen te gaan worden Antimicrobial Stewardship Programs (ASPs) ontwikkeld en ingezet. Dergelijke programma's beogen zorgverleners te ondersteunen bij het monitoren van infecties en het voorschrijven en toepassen van het juiste antimicrobiële middel, in de juiste hoeveelheid, voor de juiste periode, bij de behandeling van patiënten. Echter, door de (begrijpelijke) klinische focus gaan APSs vaak voorbii aan de gedrags- en attitudeveranderingen die zorgverleners moeten ondergaan als ze de richtlijnen willen naleven. ASPs sluiten dus niet aan bij de mensen die de interventies uit moeten voeren (de 'gebruikers') en de kliniek (de 'context'). Bovendien zijn de negatieve gevolgen van het gedrag (zoals het verkeerd voorschrijven van antibiotica) niet direct zichtbaar waardoor zorgverleners de ernst van de problematiek en hun eigen invloed hierop vaak onderschatten. Zulke attitudes en percepties van zorgverleners jegens de ernst van antimicrobiële resistentie kunnen het succes van ASPs sterk beïnvloeden.

De inzet van eHealth technologie is veelbelovend voor het omgaan met complexe situaties waarbii gedrag, attitudes en percepties van betrokkenen een belangrijke rol spelen. Dit vereist een socio-technische benadering, waarin niet de technologie centraal staat, maar juist de samenhang tussen de technologie, de mens (zorgverlener, patiënt) en de sociale en technische context (zoals werkprocessen en sociale interacties) in ogenschouw wordt genomen. Hoewel eHealth technologieën veel en vaak ingezet worden in allerlei situaties, is er nog maar weinig bekend over wat een technologie succesvol maakt. Een aantal voorwaarden zijn bekend vit eerder onderzoek. Zo is het van belang om stakeholders (belanghebbenden) actief te betrekken bij de ontwikkeling van een nieuwe technologie door middel van een participatief ontwikkelproces. Daarnaast kunnen, persuasieve elementen in de technologie de gebruikers ondersteunen en motiveren en zo een bijdrage leveren aan duurzaam gebruik van technologie. Deze kennis is toegepast in de CeHRes Roadmap, die een raamwerk biedt voor de participatieve ontwikkeling van persuasieve eHealth technologie, bijvoorbeeld door het toepassen van het Persuasive Systems Design (PSD) model.

Het onderzoek in dit proefschrift is gericht op de vraag hoe eHealth technologie zorgverleners in ASPs kan ondersteunen en daarmee de veiligheid in de gezondheidszorg kan vergroten. Daarbij ligt de focus voornamelijk op (1) hoe een participatief ontwikkelproces hieraan bij kan dragen, (2) hoe persuasief design hieraan bij kan dragen, (3) hoe het succes van persuasieve eHealth technologie geëvalueerd kan worden en (4) 
wat onze ervaringen zijn met het toepassen van de CeHRes Roadmap voor het ontwikkelen en implementeren van technologie om ASPs te ondersteunen.

In Hoofdstuk 2 zijn we als onderdeel van de Contextual Inquiry dieper ingegaan op de redenen waarom beslisondersteunende systemen in ASPs (technologie die zorgverleners ondersteunt bij het naleven van de richtlijnen voor veilig en verantwoord gebruik van antimicrobiele middelen) vaak niet nageleefd worden. Een scoping review heeft laten zien dat de huidige beslisondersteunende technologieën expert- of richtlijn-gedreven zijn. Daarnaast is gebleken dat eindgebruikers en andere stakeholders nauwelijks betrokken worden bij de ontwikkeling van de beslisondersteunende technologieën. Ook wordt weinig aandacht besteed aan het viterlijk van de technologieën. Als gevolg van het feit dat stakeholders nauwelijks betrokken waren en dat weinig aandacht wordt besteed aan het viterlijk, zijn de bestaande technologieën beperkt ondersteunend, is de adherentie suboptimaal en de implementatie daardoor problematisch.

In Hoofdstuk 3 is aangetoond hoe een participatief ontwikkelproces van een beslisondersteunende technologie voor ASP vormgegeven kan worden en op welke manier scenario-gebaseerde prototype evaluaties en een online vragenlijst hierbii van toegevoegde waarde kunnen zijn. De prototypes zijn gebaseerd op de bevindingen uit de scoping review en laten de mogelijkheden van technologie voor het ondersteunen van het voorschrijven van antimicrobiële middelen zien. Dit is gedaan als onderdeel van Contextual Inquiry en Value Specification fasen van de CeHRes Roadmap. Het uitvoeren van prototype evaluaties als onderdeel van een participatief ontwikkelingsproces stelt potentiele eindgebruikers in staat om hun manier van werken en denken te verbaliseren en om buiten hun eigen denkkaders te treden bij het formuleren van values voor nieuwe technologie. De evaluaties geven inzicht in de ervaren behoeften en verwachtingen van eindgebruikers (zoals artsen) met betrekking tot het gebruiken van technologie als ondersteuning bij het voorschrijven van antimicrobiële middelen. Eindgebruikers gaven bijvoorbeeld aan dat ze geen behoefte hebben aan ondersteuning bij de selectie van diagnostische testen. Door deze behoeften en verwachtingen ook voor te leggen aan andere stakeholders (zoals medisch-microbiologen, maar ook andere voorschrijvende artsen) kunnen ook onbekende behoeften en nieuwe inzichten in de medische praktijk aan het licht komen. Zo bleek tijdens dit onderzoek dat ondersteuning bij de selectie van diagnostische tests wel degelijk noodzakelijk was. Dit bleek vit een gebrek aan overeenstemming, zowel onder experts als onder niet-experts, over welke diagnostische testen uitgevoerd zouden moeten worden voor een casus uit de klinische praktijk.

In Hoofdstuk 4 beschrijven we dat naast het ontwikkelproces ook de karakteristieken van de technologie zelf van belang zijn voor het succes. Door de values en requirements, die zijn opgesteld tijdens een participatief ontwikkelproces, waar mogelijk te vertalen naar elementen uit het Persuasive Systems Design (PSD) model, wordt de duurzaamheid van het gebruik van en de adherentie aan technologie vergroot. In Hoofdstuk 4 is de Design fase van de CeHRes Roadmap beschreven, voor een online registratie en monitor systeem voor zorggerelateerde infecties in verpleeghuizen. Op basis van het huidige systeem voor registratie en op basis van voorwaarden, behoeften en waarden van eindgebruikers, is een doorklikbaar prototype ontwikkeld, waarin de elementen vit het PSD-model zijn opgenomen die aansluiten bij de gebruikers en de gebruikscontext. Zo is gevonden dat het registreren van cliënten veel werk was, waarbii specialisten ouderengeneeskunde veel overbodige vragen moesten doorlezen en doorklikken. Door het toepassen van Tunneling konden vragen worden overgeslagen als die voor een specifieke cliënt (op basis van 
gegeven antwoorden) overbodig bleken te zijn, waardoor registratie efficiënter is geworden. Scenario-gebaseerde evaluaties zijn uitgevoerd om in kaart te brengen welke persuasieve elementen op welke manier van toegevoegde waarde zijn. Deze manier van evalueren stelde de eindgebruikers in staat om aan te geven wat zij als prettig en ondersteunend ervaren. Bovendien bleek dat persuasieve technologie artsen kan ondersteunen bij het sneller, gemakkelijker en preciezer vitvoeren van hun taken.

In Hoofdstuk 5 beschrijven we dat naast de participatieve ontwikkeling ook een voortdurende evaluatie van belang is om de technologie continu te blijven verbeteren. Om te evalueren of en welke persuasieve elementen in persuasieve technologie een effect hebben op het succesvol ondersteunen van ASPs, zijn gevalideerde evaluatiemethoden nodig. Om te evalueren in hoeverre technologie als persuasief wordt ervaren, is de Perceived Persuasiveness Questionnaire (PPQ) ontwikkeld, maar deze is nog niet gevalideerd. Het lijkt een veelbelovende manier voor zowel Formatieve als Summatieve Evaluaties in de CeHRes Roadmap. Om het potentieel van de PPQ te onderzoeken, is de ervaren persuasiviteit van een eHealth technologie onderzocht met behulp van drie methodes (PPQ, expert evaluatie en gebruikersevaluaties m.b.v. een prototype) waarvan de resultaten achteraf vergeleken zijn. Uit deze vergelijking is gebleken dat de resultaten van de PPQ consistent zijn met de resultaten die via de andere methoden zijn verkregen: PSD-principes die volgens experts sterk aanwezig waren in de technologie, zijn ook ervaren door de potentiële eindgebruikers die de PPQ in hebben gevuld, en deze principes zijn ook herkend in het gebruikersonderzoek. Dit onderzoek liet echter ook zien dat er nog ruimte voor verbetering is voor een aantal PPQ-constructen en items. Een sterke overlap is gevonden tussen Primaire Taak Ondersteuning en Ervaren Effectiviteit. Verder onderzoek is nodig om te onderzoeken of deze en andere constructen samengevoegd of op een andere manier gewijzigd zouden moeten worden. Eerdere onderzoeken hebben laten zien dat de PPQ weliswaar nog niet gevalideerd maar wel veelbelovend is. Validatie van deze vragenlijst is dus van toegevoegde waarde.

In Hoofdstuk 6 zijn de eerste stappen voor de ontwikkeling van een gevalideerde vragenlijst voor de evaluatie van persuasiviteit gezet. Een card sort studie is gecombineerd met het invullen van de PPQ om de constructvaliditeit en de interne consistentie van de $P P Q$ te evalueren. Hoewel een card sort meestal gebruikt wordt voor het bepalen van de informatiestructuur van een systeem, is het in dit onderzoek succesvol toegepast voor de evaluatie van de structuur van een vragenlijst. De resultaten onderschrijven de relevantie en urgentie van het ontwikkelen van een gevalideerde methode om de persuasiviteit van een technologie te evalueren. De meeste PPQ-constructen zijn bevestigd door een cluster analyse. Echter, de resultaten van de analyse laten zien dat de interne consistentie van de vragenlijst wordt vergroot door het samenvoegen van de constructen Primary Task Support, Perceived Persuasiveness en Perceived Effectiveness in een enkel construct: Primary Goal Support. Vervolgstappen (zoals betere onderbouwing van de constructen en een factoranalyse) zijn nog nodig om de PPQ nader te valideren.

In Hoofdstuk 7 is teruggekeken op de ervaringen met het ontwikkelen en implementeren van eHealth technologie via de CeHRes Roadmap om ASPs te ondersteunen. We beschrijven hoe eHealth technologie ontwikkeld en geïmplementeerd kan worden om de kernelementen van een ASP te ondersteunen, zoals die beschreven staan in het rapport van de Centers for Disease Control and Prevention (CDC). In de Contextual Inquiry zijn een scoping review en scenario-based prototype evaluaties uitgevoerd met betrekking tot beslisondersteunende systemen (zie ook Hoofdstukken 2 en 3). Hieruit bleek dat huidige 
systemen weinig ondersteunend zijn en zijn manieren beschreven om deze ondersteuning te verbeteren (bijvoorbeeld door informatie te geven over geschikte antimicrobiële middelen in het geval van een allergie). De Value Specification en Operationalization fasen zijn beschreven met behulp van de casus van het Maturity model voor ASPtechnologieën. Via business modelling zijn de belangrijkste stakeholders geïdentificeerd en hun waarden in kaart gebracht. Op basis daarvan is een Maturity model opgesteld. Dit model geeft aan welke ASP-interventies de grootste toegevoegde waarde hebben bii het verbeteren van een ASP, op basis van de huidige status van het ASP in een instelling. De Design fase is geïllustreerd via twee verschillende technologieën. Tijdens de Designfase van de Verpleegkundigen Informatie app (waarvoor prototype evaluaties en card sorts vitgevoerd zijn) leerden we dat het van belang is om de werkprocessen van de eindgebruikers van een technologie te doorgronden en het system en de inhoud aan te laten sluiten binnen deze context. In dit geval betekende dit dat informatie gestructureerd en op een manier die past bij de werkprocessen van de verpleegkundigen (bijvoorbeeld voorbereiden, toedienen en monitoren van de therapie) aangeboden moet worden. Tijdens de Design-fase van de Prevalentie app (zie ook Hoofdstuk 4) zijn scenariogebaseerde prototype evaluaties uitgevoerd om te onderzoeken welke soorten persuasieve elementen ondersteuning kunnen bieden bij het uitvoeren van prevalentiemetingen door specialisten ouderengeneeskunde. Dit vereist dat de technologie hen ondersteunt om dit zo snel, betrouwbaar en eenvoudig mogelijk te kunnen doen. We hebben daarnaast ook geleerd dat prototype-evaluaties heldere inzichten geven in welke persuasieve elementen gewaardeerd worden door eindgebruikers. Onderdeel van het prototype was de mogelijkheid om de registratie te pauzeren en op een later moment te hervatten, zonder dat data verloren gingen. Eindgebruikers gaven aan dat dit het veel makkelijker maakte om deze taak in hun werkprocessen te integreren.

In Hoofdstuk 8 zijn de resultaten van de vorige hoofdstukken bediscussieerd en zijn implicaties voor ontwikkeling en ontwerp van eHealth technologie voor ASP besproken. Daarnaast zijn aanbevelingen gedaan voor vervolgonderzoek. De eerste onderzoeksvraag van dit proefschrift was: Hoe kan een participatief ontwikkelproces van eHealth technologie artsen ondersteunen in een ASP? In dit proefschrift hebben we laten zien dat een participatief ontwikkelproces (waarin gebruik wordt gemaakt van prototype evaluaties) inzicht geeft in de gebruikers en hun denk- en werkprocessen, waardevolle inzichten oplevert in wat een technologie zou moeten doen en hoe het er vit zou moeten zien. Daarnaast moeten ook richtlijnen en protocollen altijd scherp in het oog worden gehouden tijdens de ontwikkeling van technologie, omdat zij de medische/klinische inhoud vertegenwoordigen, die met de technologie gecommuniceerd moet worden. We bevelen dan ook aan om niet alleen eindgebruikers, maar ook andere stakeholders te betrekken en richtlijnen scherp in het vizier te houden bij de ontwikkeling van eHealth technologie om ASPs te ondersteunen.

De tweede onderzoeksvraag van dit proefschrift was: Hoe kan persuasief design van eHealth technologie artsen ondersteunen in een ASP? Dit proefschrift heeft laten zien dat het combineren van een participatief ontwikkelproces met elementen van het Persuasive Systems Design (PSD) model de kansen op duurzaam gebruik van en adherentie aan de technologie vergroot. Dit proefschrift heeft laten zien hoe zo'n combinatie vormgegeven kan worden en wat de toegevoegde waarde daarvan is. Dit is gedaan via de casus van een technologie voor web-gebaseerde registratie en monitoring van zorggerelateerde infecties in verpleeghuizen. Op basis van de resultaten in dit proefschrift raden we aan om de keuze en toepassing van persuasieve elementen af te laten hangen van het doel, 
de gebruiker en de context van een specifieke technologie. Daarnaast moet de gepastheid van de gekozen persuasieve elementen altijd getest worden bij stakeholders.

Naast de participatieve ontwikkeling van technologie is ook de evaluatie van groot belang om de technologie blijvend te kunnen innoveren en verbeteren. Daarom was de derde onderzoeksvraag van dit proefschrift: Hoe kan het succes van persuasieve eHealth technologie geëvalueerd worden? De PPQ bleek een veelbelovende methode te zijn om de ervaren persuasiviteit van technologie te evalueren, hoewel de vragenlijst nog aanpassingen behoeft om de validiteit te verbeteren. Eerder onderzoek heeft laten zien dat de constructen uit het PSD-model van toepassing zijn op veel verschillende technologieën, die gebruikt worden door verschillende eindgebruikers en in verschillende settings. Echter, de evaluatie in dit proefschrift heeft laten zien dat items van de PPQ niet altijd geschikt zijn om de elementen te meten die ze nu zouden moeten meten en dat daar soms overlap in zit. Met andere woorden, er is niet altijd een 1-op-1 relatie is tussen de elementen vit het PSD-model en de items van de vragenlijst. Een belangrijke aanbeveling op basis van dit proefschrift is daarom om de items van de PPQ en de samenhang met de elementen uit het PSD model te heroverwegen. Op dit moment wordt elk element uit het PSD-model bevraagd met behulp van meerdere items die elk een verschillend aspect van het desbetreffende element bevragen. Om een meer generieke en valide vragenlijst te ontwikkelen wordt aanbevolen om elk element te bevragen door middel van 2 of 3 items die hetzelfde aspect van het construct uitvragen en dus sterk overeenkomen.

De vierde en laatste onderzoeksvraag van dit proefschrift was: Wat hebben we geleerd van het toepassen van de CeHRes Roadmap bij het ontwikkelen en implementeren van eHealth technologie om ASPs te ondersteunen? In dit proefschrift hebben we laten zien dat onze aanpak bijdraagt aan de ontwikkeling van technologie die past bij de eindgebruiker, bii de gebruikscontext en bii de huidige status van de ASP in een instelling. Daarnaast levert onze aanpak nieuwe inzichten op in de werk- en denkprocessen van eindgebruikers en kunnen zij via onze aanpak gestimuleerd worden om buiten de eigen referentiekaders te denken over oplossingen.

Vervolgonderzoek is nodig om te bepalen wat de lange termijn en klinische effecten zijn van het toepassen van de geformuleerde aanbevelingen. Daarnaast is aanvullend onderzoek nodig om een 'nieuwe PPQ' te formuleren en verder te valideren voo r gebruik in een ontwikkelingscontext en voor summatieve evaluatie. 

Dankwoord 
De zin "ik doe promotieonderzoek" vind ik één van de vreemdste zinnen in de Nederlandse taal. Ten eerste omdat ik me nooit heb kunnen voorstellen dat er ooit een boekje zou bestaan waarin ik vier jaar onderzoek heb kunnen en mogen beschrijven. Ik vind het nog steeds ongelooflijk dat ik dat heb mogen doen. Wat een luxe om je vier jaar lang bezig te mogen houden met dat wat je interessant en leuk vindt (en daarnaast met nog een miljoen andere dingen, maar dat is weer een ander verhaal). Maar ik vind "ik doe promotieonderzoek" vooral een rare zin omdat de "ik" in deze zin vit een ongelooflijke hoeveelheid mensen bestaat. Zii hebben elk op zijn of haar eigen manier en in verschillende mate bijgedragen aan dit onderzoek en aan dit proefschrift. Het schrijven van het dankwoord is daarom het onderdeel waar ik me al vier jaar op verheug, maar waar ik ook al vier jaar tegenop zie. Want wat nou als ik iemand vergeet te noemen?! Nou ja, dan hoop ik maar dat ik 'live' mijn waardering voor uw/jouw hulp al voldoende heb laten blijken. Want dat die waardering groot is, voor alle hulp die ik heb gekregen bii de totstandkoming van 'mijn' boekje, staat buiten kijf.

Lisette, mijn promotor, hoe kan het ook anders dan dat ik dit dankwoord start met mijn waardering voor jou. Tijdens mijn studie Health Sciences kwam ik voor het eerst in aanraking met het vak eHealth, met het MRSA-net project en met het fenomeen Lisette van Gemert-Pijnen. In de loop der jaren heb ik onderzoeksopdrachten voor verschillende vakken in mijn opleidingen (Health Sciences en Health Psychology), twee afstudeeropdrachten, een studentassistentschap en viteindelijk mijn promotie onder jouw supervisie mogen doen. En super(veel)visie heb je zeker! De keren dat je enthousiast mijn kamer binnenstormde (je begint doorgaans tijdens het binnenlopen op de gang alvast te praten) met nieuwe ideeën en mogelijkheden zijn echt niet meer te tellen. En dan heb ik het nog niet eens over de keren dat je alleen even binnen kwam vallen om een dropje te halen. Doorgaans onder vermelding dat je 'binnenkort echt een zakje voor ons meeneemt'. Al die nieuwe ideeën betekenden ook dat het meer dan eens is voorgekomen dat we toch nog even ad hoc een artikel of boekhoofdstuk in elkaar moesten zetten. Wat een kick als dat dan toch weer lukte! En wat hebben we de afgelopen jaren met jouw eHealth club veel meegemaakt, plezier gehad en wijze lessen geleerd (op hoge hakken door Venetië is geen goed plan en als het $>30^{\circ} \mathrm{C}$ is kun je in Barcelona beter een taxi naar je hotel nemen als je koffers mee moet slepen...). Ik wil je bedanken voor de kansen, vrijheden en steun die je me hebt gegeven en blijtt geven!

Dan mijn copromotor Robbert Sanderman, zo ongeveer een tegenpool van Lisette en juist daardoor zo ontzettend waardevol voor mijn project. Je sloot wat later aan bij het project en bracht rust, structuur en overzicht. Ik zal nooit vergeten dat je ooit tegen me zei: "ga nou eens gewoon naar buiten zitten kijken". Toen ik dat dan maar eens probeerde, zat ik vooral te denken: "Tjonge die ramen moeten echt eens schoongemaakt worden... hé wat hoor ik daar? Och wat leuk, een eendje!"... Gelukkig bleek ook het bestuderen van die eendjes te helpen om ideeën te krijgen over hoe ik mijn verhaal kon vertellen. Ik wil je dan ook ontzettend bedanken voor je wijze raad, voor je humor en vooral dat je er goesting in had om me te begeleiden bij het afronden van mijn proefschrift.

Alex Friedrich, copromotor maar vooral MRSA-net, EurSafety en Health-i-care goeroe! Ontzettend bedankt voor je enthousiasme over het onderzoek dat we in Twente doen (toch een beetje de vreemde eend in de bijt bij de meeste projecten). Toen ik als student voor het eerst kennis met je maakte, was ik direct onder de indruk van je passie voor wat je doet. Je bezit een ongekend talent om die passie ook over te brengen op de mensen om je heen. Ik ben ervan overtuigd dat dit een grote bijdrage heeft geleverd aan het grote 
succes van de projecten. Ik hoop dat ik, als ik later groot ben, een beetje van dat talent heb mogen leren.

I am very grateful that the members of my graduation committee were willing to spend time and energy to read my thesis, therewith enabling my promotion. Prof. dr. Harri OinasKukkonen, Prof. dr. Jacques Scheres, Prof. dr. Bhanu Sinha, Dr. Ron Hendrix, Prof. dr. Hermie Hermens, Dr. Carine Doggen, thank you for being part of the committee. Special thanks to Prof. dr. Harri Oinas-Kukkonen for traveling all the way from Finland to attend and participate in my promotion.

De EurSafety bende uit Twente. 'Intens' is het woord dat me het eerst te binnen schiet als ik denk aan onze overleggen toen we nog compleet waren. Wat een interessante mix van mensen zijn we en wat maakt dat het samenwerken interessant. Lisette, die altijd 20 stappen verder was dan de rest (soms ook een totaal andere afslag had genomen, liefst zonder dat de rest dat wist). Ron, die altijd (altijd!) tegengas gaf en het belang van de kliniek scherp in het oog hield. Dan Jobke, Joyce, Maarten en ik die de chaos compleet maakten met onze ideeën en praktische bezwaren ("ja, hartstikke leuk, maar hoe gaan we dat dan doen?!"). Behalve dat we tijdens deze overleggen een nieuwe standaard voor het woord 'discussie' hebben gezet, hebben we ook veel van elkaar kunnen leren en veel gelachen. Ik wil jullie daar allemaal heel hartelijk voor danken. Lieve Jobke, onze onderzoeken sloten erg mooi bij elkaar aan, wij hebben dan ook behoorlijk intensief samengewerkt. Dat begon tijdens mijn afstudeeropdracht en daarna toen ik als studentassistent samen met jou de MRSA review mocht doen. Wat is het een genot om al die jaren samen te kunnen werken met iemand met wie ik niet alleen ontzettend fijn kan sparren over onderzoek (en oh wat zijn we fijn kritisch), maar met wie ik ook buiten het werk erg goed kan opschieten. Wat heerlijk om ook de vraagstukken uit ons privéleven (en de combinatie daarvan met werk) samen te kunnen bespreken en beleven. Toegegeven, niet al onze gezamenlijke beslissingen waren even succesvol (weggaan bij een bijeenkomst vlak voordat er een Michelinsterwaardig diner wordt geserveerd is geen goed idee. Oef, het doet nog steeds pijn). Maar daarvoor geldt: gedeelde smart is halve smart. Ik wil je niet alleen bedanken, maar onze samenwerking vooral ook voortzetten. Gelukkig hebben we afgesproken dat we minstens één keer per jaar een projectje samen oppakken. Nu al een goed plan!

Mocht $u$ mijn thesis daadwerkelijk gelezen hebben (waarvoor dank!), dan is het u vast opgevallen dat dit boekje niet mogelijk zou zijn geweest zonder een heleboel 'stakeholders' die deel hebben genomen aan mijn verschillende onderzoeken. Hun input, hun bereidheid om tijd te investeren, is van onschatbare waarde geweest voor dit project. Hierbii verdienen de verpleeghuisartsen van de verschillende verpleeghuizen in de regio Nijmegen, DIPpers (o.a. Lian, Ellen) en arts-assistenten van MST, de enthousiastelingen van de afdeling Medische Microbiologie van het UMCG (Alex, Bhanu, Jerome, JanWillem, Piet, Johan, e.v.a.) en alle studenten die deel hebben genomen aan het PPQ onderzoek een bijzondere vermelding.

Liisa Kuonanoja, you were willing to help me by collecting data for the PPQ research among Finnish students. Thanks so much for your enthusiasm and for our very smooth and pleasant cooperation in the PPQ research. We loved having you here and I loved how much you loved Dutch houses! I am sure we will be seeing you in the future. 
Andrea Eikelenboom-Boskamp, we hebben samen veel hordes overwonnen tijdens de ontwikkeling en implementatie van de Prevalentie app. Bedankt voor je inzet en voor je motivatie om alles tot een goed einde te brengen.

Ook mijn zeer gewaardeerde (oud)collega's van de eHealth groep verdienen een eervolle vermelding. Niet alleen door de inhoudelijke (en soms ook inhoudsloze) gesprekken en discussies tijdens onze traditioneel chaotische overleggen en congres bezoeken. Maar ook omdat we allemaal weten dat we gewoon even bij elkaar binnen kunnen lopen om iets te vragen, even te overleggen, overwinningen te vieren of dompers te delen. Wat geweldig dat we in een wetenschappelijke wereld, waar concurrentie vaak een grote rol speelt, toch zo goed met elkaar kunnen samenwerken, elkaar het succes gunnen en met elkaar meedenken. Lisette, Aniek, Anne, Annemarie, Aranka, Britta, Floor, Hanneke, Hans, Jobke, Joyce, Liseth, Maarten, Moniek, Olga, Saskia A, Saskia K heel erg bedankt en laten we dat vooral volhouden! Floor, iij en ik hebben binnen de eHealth groep nog wat meer met elkaar samen mogen werken, schrijven en leren. We hebben van alles ondernomen. Variërend van het begeleiden van studenten tot het volgen van een cursus programmeren voor vrouwen (want daar kwam het eigenlijk op neer) in Groningen, ook samen met Jobke. Dat volgen van die cursus ging overigens niet zonder slag of stoot. Ik zal geen namen noemen, maar één van ons drieën heeft in Groningen gewoond, dus Jobke en ik (ik laat het aan uw deducerend vermogen over om vast te stellen wie er dan in Groningen heeft gewoond) gingen er vanuit dat die persoon ons naar het Hampshire hotel zou navigeren. Dat is ook bijzonder soepel gelukt... ware het niet dat er dus 2 van die hotels zijn in Groningen en wij bij de verkeerde stonden. Nou ja, het is maar een voorbeeld. Maar wel een exemplarisch voorbeeld voor de (zelf gecreëerde) avonturen die we samen hebben beleefd. Ik wil je ontzettend bedanken voor de samenwerking, maar vooral voor de vriendschap die daar vit voort is gekomen. We hebben vele ups en downs beleefd in de afgelopen jaren, wat fijn dat we die altijd bij een grote mok thee of een heerlijke burger konden bespreken.

Rookies (a.k.a. broekies)!!! Wat fijn dat jullie er zijn! Tijdens onze onregelmatig regelmatige overleggen bespreken we alles wat met het aio-leven te maken heeft. Problemen worden besproken, artikelen geëvalueerd, posters beoordeeld. Maar er wordt vooral heel veel gedeeld: kennis en ervaring, maar ook oplossingen en plezier. Hoewel die kennis en oplossingen soms ver te zoeken lijken... het blijft toch best pijnlijk dat we gewoon collectief NIET ontsnapt zijn vit de Escape rooms (of, zoals Annemieke het zei: "Toen we hier naar binnen gingen dacht ik nog: nou, we zijn toch allemaal bovengemiddeld intelligent, dus dit moet goedkomen"... Tja!). (Oud)PGT rookies Aniek, Anne, Annemieke, Aranka, Carly, Farid, Floor, Ingrid, Jobke, Jochem, Laura S, Laura W, Maarten, Mareike, Marijke, Marion, Marit, Martijn, Melle, Moniek, Nadine, Noortje, Petra, Robin, Saskia A, Saskia K, Teuntje, Wouter, Youri heel erg bedankt voor alles!

Alle collega's van PGT wil ik bedanken voor de fijne samenwerking en sfeer. ledereen is altijd bereid om even mee te denken over onderzoek, over de begeleiding van studenten of om gewoon even een gezellig praatje te maken. Ik heb van al die vormen van samenwerking de afgelopen jaren dan ook dankbaar gebruik gemaakt. Speciale dank voor Ernst Bohlmeijer, omdat je me ook interessante en leuke kansen gaf om me naast mijn aioschap te ontplooien: de coördinatie van het PGT colloquium, het organiseren van het vakgroepsuitje (hoe vervelend was dat, Marloes en Marjolein!?), zitting in de IGS Instituutsraad, en nog veel meer... ik vond het allemaal even leerzaam (ok, het vakgroepsuitje misschien iets minder, maar dat was dan weer gewoon knetterleuk)! 
Robbert en Ernst: bedankt voor jullie vertrouwen dat we met het 'designteam' onze gangen in de Cubicus mochten pimpen. We (Marcel, Nadine en ik) vinden zelf in ieder geval dat het helemaal geslaagd is. Ik hoop dat we zo een kleine bijdrage kunnen leveren aan het nog beter vitdragen van het fantastische onderzoek dat we doen. Ook ons secretariaat (Marieke, Elize, Marja, Marion) heel erg bedankt voor jullie steun en hulp bij kleine en grote vragen. Marieke, het organiseren van een congres is toch een stuk leuker als iii je er mee bemoeit!

En daar zijn ze dan eindelijk: mijn buddies, mijn kamergenootjes, mijn paranimfen! Lieve Laura, je bent mijn super attente tegenpool. Wat is het toch vreemd als er vit jouw altijd vriendelijke mond ineens een Nienke-achtige sarcastische opmerking kwam. Laat je niet besmetten! :) Je aandacht voor details zie je niet alleen terug in je werk, maar ook in je privéleven. Waar ik in staat ben om iemands verjaardag te vergeten terwijl die zelfs (inclusief bijzonder persuasieve reminder) in mijn agenda staat, denk iij aan alle kleine en grote gebeurtenissen in het leven van de mensen om je heen. Altijd heb je dan een lief woord, een attent kaartje of een mooi gebaar. Toen ik een cursus Duits (jawel) ging doen in Enschede, heb ik wekenlang iedere dinsdag bij Frank en jou mogen eten. Ik heb veel van jouw open en gastvrije houding geleerd (dat hoop ik althans) en wil je daar heel erg voor bedanken! Lieve Nadine, mijn maatje, als ik later een scheefgegroeid oud dametje ben, weet ik in ieder geval waar het door komt. Alles, en dan bedoel ik ook alles, bespraken we de afgelopen jaren terwiil ik naar links hing en iii naar rechts, om elkaar langs onze pc-schermen aan te kunnen kijken. Waarom dat nooit langs de andere kant gebeurde snapten we zelf ook eigenlijk niet, maar toen we het eens vitprobeerden voelde het toch niet goed. Zouden we al scheef gegroeid zijn? lk vind het in ieder geval een prachtige metafoor voor onze vriendschap: hoe krom we soms ook redeneren, de ander begrijpt het moeiteloos. Who knew dat puppies en babies zo op elkaar leken?! Ik wil je ontzettend bedanken voor wie je bent en voor wie je bent voor mij. Ik ben heel blij dat we voorlopig nog roomies kunnen blijven! Meiden, zonder jullie steun had ik dit promotieavontuur niet zo fijn af kunnen ronden, ik vind het daarom een grote eer dat jullie ook tijdens de allerlaatste momenten van mijn promotietraject naast mij zullen staan als mijn paranimfen. Alles Liebe, Eure Nienke.

Dan zijn er nog een heleboel studenten die ik als eerste of tweede afstudeerbegeleider heb mogen volgen in hun traject van bachelorstudent naar Bachelor of Science, of van masterstudent naar Master of Science. Hun projecten hebben op directe of indirecte wijze een bijdrage geleverd aan dit proefschrift. Door data te verzamelen die hier zijn gebruikt of door een interessant zijpad te bewandelen, waar ik zelf tijdens dit project helaas geen tijd voor had. Marieke, Esther, Rianne, Charlie, Kiki, Lisa, Natalia, Noreen, Tina, Diana, Christian, Derk-Jan, Triston, Vivien, Felicity, Deike en Lieke bedankt voor jullie inzet en voor jullie harde werken.

Hebt $u$ de omslag van mijn proefschrift gezien? Tof gemaakt he!? Ik ben er in ieder geval ontzettend blij mee. Het is de heren van Buro Bam! op miraculeuze wijze gelukt om mijn enthousiaste maar warrige ideeën en kreten om te zetten naar een boekomslag die niet alleen mijn verhaal vertelt, maar die ook echt 'Nienke' is. Thijs en Rob, heel erg bedankt voor jullie geïnteresseerde en kritische vragen en voor de enorm kundige, fijne en gezellige manier waarop jullie dit voor mij tevoorschijn hebben getoverd!

Terwijl ik dit schrijf, zo tijdens de laatste weken voor de deadline van het indienen van mijn manuscript, zou ik het bijna vergeten... maar er is ook nog zoiets als een leven buiten het werk. Zonder de steun die ik daar kreeg, was alle steun op het werk ijdele hoop geweest. 
Er zijn een heleboel mensen in mijn leven geweest die hebben moeten wennen aan het feit dat er simpelweg geen hersencapaciteit meer over was voor niet-werkgerelateerde dingen. Zij verdienen daarom een ereplaatsje in dit dankwoord. Een aantal van hen wil ik in het bijzonder noemen.

Lieve meiden van de blije-eitjes club (Mirjam, Maaike en Mariska, voorzitter Irma en faciliterende Onnie), heel erg bedankt voor de uurtjes, appjes, zorgen, mokken thee, blunders en wijsheden die we (eerst noodgedwongen, later met heel veel plezier) met elkaar konden delen. Wat fijn dat jullie er zijn als niet-werk gerelateerde klankbordgroep. Het was en is telkens even een rustmomentje geweest in de hectische academische- en mama-werelden waarin we ons allemaal begeven. Gelukkig weet ik zeker dat er nog vele appjes, zorgen, mokken thee, blunders en wijsheden zullen volgen!

Lieve Hanneke en Marleen, mijn volleybuddies, mijn fröbelclubgenootjes. Wat zijn jullie ontzettend lief, streng, begripvol en meelevend geweest. De laatste (vele) weken had ik nauwelijks tijd om jullie te zien. Dat vonden jullie nooit een probleem en via appjes enz hielden jullie me op de hoogte van wat er in jullie levens gebeurde. Toch wisten jullie te onderhandelen om dan maar een keer 's avonds een ijsje te gaan eten samen ("en dan niet meer daarna nog de computer aanzetten Nien"). Tia, voor een ijsje ben ik natuurlijk altijd te porren. En lieve mooie Leen, wat een bijzonder ijsje is dat geworden. Het is het eerste ijsje in mijn leven dat ik nooit ga vergeten. Dex en ik hebben onze fiets al klaar staan voor de toekomst! Lieve Hannes, mijn zusje dat geen zusje is. Wat hebben wij al bijzondere momenten met elkaar mogen delen. Momenten die ik altijd bij me draag en waar ik vaak en met heel veel blijdschap aan terugdenk. Je bent geweldig omdat je super lief bent, maar ook omdat je gewoon tegen me zegt als ik iets belachelijks uitkraam (ook al is het dan natuurlijk helemaal niet belachelijk). Ik wil jullie bedanken voor jullie vriendschap, maar ook omdat we op het volleybalveld coping strategieën hebben ontwikkeld die ook voor promoveren bijzonder nuttig zijn gebleken. Op naar nog vele jaren in een happy thoughts bubble!

Lief dagboek 2.0... Freek, Hayo en Heike, samen hebben we onze studie Health Sciences doorlopen. Vele uren hebben we samen op projecten zitten ploeteren en voor tentamens zitten stampen. Maar ook daar buiten was het altijd gezellig met ons traditionele jaarlijkse bezoek aan een pretpark als bekroning. Mag ik daarbij overigens opmerken dat dat weer hoog tijd wordt! Bedankt voor alle jaren gedeeld 'studieleed' en voor alle gezelligheid.

Lieve Nadine, Laura, Floor en Jobke. Jullie zijn weliswaar al genoemd, maar ik verkeer in de heerlijke positie dat ik samen mag werken met een aantal collega's die inmiddels ook heel dierbare vriendinnen zijn geworden. Behalve mooie projecten op het professionele vlak, heeft dat ook juweeltjes van projecten opgeleverd in de privésfeer: Hoe gaan we dat telefoonnummer fixen zonder dat we hier nooit meer kunnen eten? Hoe krijg je een witte muur zo vlekkerig mogelijk aubergine kleur (of was dat nou juist niet te bedoeling Nadine)? Zijn er nog locaties waar we de high-tea nog niet hebben vitgeprobeerd? Overigens verstaan we ook de kunst van de grensoverschrijdende aanpak tot in de puntjes: samen hebben we heel wat congressen bezocht (Groningen, Amsterdam, Venetië, Londen, Barcelona en Berlijn schieten me zo te binnen) en georganiseerd. Daarbii wisten we altijd wel een mogelijkheid te vinden om nog even van de omgeving te genieten. Toppunt was wat mij betreft het congres in Barcelona, waar Laura en Nadine ook nog even een weekendje voor de gezelligheid kwamen. Ik wil jullie bedanken voor jullie dierbare vriendschap, maar ook omdat we samen de definitie zijn van 'het nuttige met het aangename verenigen'. 
En last maar zeker niet least mijn familie, mijn roots, mijn basis. Lieve allemaal, dankzij jullie ben ik geworden wie ik ben. Opgegroeid in onze kleine maar knettergekke familie. Dankjulliewel voor alle fijne momenten en voor alle steun.

Lieve opa, wat leef je toch altijd mee met mij en met al je kleinkinderen. Ik zal nooit vergeten dat ik mijn afstudeerscriptie bij jullie had laten liggen en dat ik het later terugzag met allerlei vragen in de kantlijn geschreven. Je had het helemaal gelezen! Dat wordt nu en met dit boekje wat lastiger, maar ik weet dat je altijd net zo geïnteresseerd en trots zult blijven. Dankjewel dat je me dit gevoel hebt gegeven, ik zal dat de rest van mijn leven bij me dragen als een dierbaar bezit. Lieve oma, ondanks je drukke leven vraag je me altijd hoe het gaat met mijn 'afstuderen'. Wat bijzonder dat je daar altijd oog voor hebt en houdt. Dankjewel dat je het altijd blijft vragen en dankjewel dat je altijd bezorgd over me bent (maar dat hoeft niet hoor!).

Lieve Marty en Wilhelmien, jullie zijn fantastische schoonouders. Dankjulliewel voor jullie niet-aflatende belangstelling voor alles in mijn leven, vanaf de eerste dag dat ik dit heb mogen delen met jullie zoon. Jullie zijn nu bijna de helft van mijn bestaan onderdeel van mijn leven. En zo voelt dat ook. Bedankt dat jullie me het gevoel geven dat ik niet alleen in naam bii de Beerlage-clan hoor.

Lieve Sander, Marloes, Noortje en Eefje. Jullie wonen helaas niet om de hoek, maar als we jullie zien genieten we optimaal van de vrolijke chaos die jullie met je meebrengen. Mooie, lieve Noortje en Eefje, nog even en dan kunnen jullie Dex ook alle knappe dingen leren die jullie al kunnen! Wat fijn dat Dex en wij zulke lieve grote nichtjes hebben.

Lieve stoere grote broer, lieve Pelle. Wat is het fijn dat ik met jou op heb mogen groeien. Samen hebben we menig boompje beklommen, rondje om het veld gerend (en gewonnen, maar dat terzijde!), robbertje geknokt (helaas standaard door mij verloren) en kikkervisje gevangen. We komen uit hetzelfde nest maar zijn toch zo verschillend. Samen zouden we een soort Iron (wo)Man kunnen zijn! Volgens jou heb ik de brains en volgens mij heb iii alle fysieke talenten... wat een team! Lieve Nina, wat fijn dat je er bent voor mijn grote broer en wat gezellig dat je deel bent geworden van onze familie. Bliif maar lekker zo eigenwijs als je altijd bent, heerlijk!

Lieve papa en mama. Ik weet niet zo goed wat ik hier voor jullie moet opschrijven. Ik ben onbeschrijfelijk gek op jullie en nog onbeschrijflijker dankbaar voor alle kansen en aanmoedigingen die jullie me hebben geboden. Het is een understatement om te zeggen dat dit me niet gelukt was zonder jullie steun. Maar dat geldt eigenlijk voor alle dingen in mijn leven waar ik trots op ben. Daarom beperk ik me er maar toe om uit de grond van mijn hart te zeggen: Dank Jullie Wel.

Dan mijn lieve, allerliefste mannen. Jullie zijn eerste en laatste auteur van iedere vezel van dit proefschrift. Lieve Jeroen, samen hebben we al heel wat hoogte en dieptepunten doorleefd. Ik geloof dat de totstandkoming van dit proefschrift daar een miniatuurversie van is geweest. De afgelopen jaren hebben mensen vaak tegen me gezegd dat promoveren vast niet makkelijk is en vast veel toewijding vergt. Dat is een waarheid als een koe. Maar ik geloof dat dit minstens net zozeer geldt voor getrouwd zijn met een promovenda. Wat ben ik een bofkont dat ik altiijd weet dat iij er bent als ik een luisterend oor, een dikke knuffel of gewoon even rust nodig heb. Wat ben ik een bofkont dat iij er bent. De afgelopen jaren zijn we naast de promotieavonturen nog heel wat andere grote avonturen aangegaan: we hebben een huis gekocht (in 2013), we zijn getrouwd (in 2014) en bovenal hebben we samen (in 2015) een prachtig kindje gekregen. In 2016 even 
promoveren en dan beloof ik dat we in 2017 je verjaardag eindelijk weer echt gaan vieren! (-) Dankjewel voor ons, ik heb zin in de toekomst. Lief sloopkogeltje, lief knuffelbeer(lage)tie, lieve Dex. Wat ben ik blii dat iii er bent en wat maak je het ons makkelijk met je altijd goede humeur, je stralende lach en je boevenoogies. Zonder dat je er ook maar iets van begreep, was je de eerste die mijn manuscript goedkeurde. Terwiil ik thuis op de bank de discussie zat te schriiven, speelde iii gierend van het lachen kiekeboe om het scherm van mijn laptop heen. Terwiil ik probeerde mijn manuscript door te lezen om zo veel mogelijk fouten eruit te halen, was het jouw persoonlijke missie om zoveel mogelijk bladzijden uit de map te scheuren en die zo goed mogelijk te verkreukelen. Ik kan me geen betere reden voorstellen om wat onvermijdelijke foutjes in het manuscript over het hoofd te zien. Ik ben benieuwd naar alle avonturen die nog op ons te wachten staan. Maar samen met je papa heb ik er ontzettend veel zin in!

Het is geluk(t)!!! 

Publications and other output 


\section{Publications and other output}

Journal articles [peer-reviewed]

Wentzel, M.J., Müller, F., De Jong, N., \& Van Gemert-Piinen, J.E.W.C., Card sorting to evaluate the robustness of the information architecture of a protocol website.

International Journal of Medical Informatics, 2016, 86, p. 71-81.

Beerlage-de Jong, N., Eikelenboom-Boskamp, A., Voss, A., Sanderman, R. \& Van

Gemert-Pijnen, J.E.W.C., Combining User-Centered Design with the Persuasive Systems

Design Model; The Development Process of a Web-Based Registration and Monitoring

System for Healthcare-Associated Infections in Nursing Homes. International Journal on Advances in Life Sciences, 2014, 6(3\&4), p. 262-271.

Wentzel, M.J., De Jong, N., Niidam, L., Van Drie-Pierik, R., \& Van Gemert-Pijnen, J.E.W.C., Understanding eHealth use from a Persuasive System Design perspective: an Antibiotic Information Application for Nurses. International Journal on Advances in Life Sciences, 2014, 6(3\&4), p. 210-219.

Wentzel, M.J. Van Velsen, L., Van Limburg, M., De Jong, N., Karreman, J., Hendrix, R. \& Van Gemert-Pijnen, J.E.W.C., Participatory eHealth development to support nurses in antimicrobial stewardship. BMC medical informatics and decision making, 2014, 14(45).

\section{Book chapters}

Wentzel, M.J., De Jong, N., Karreman, J. \& Van Gemert-Pijnen, J.E.W.C. Implementation of MRSA Infection Prevention and Control Measures - What Works in Practice? In:

Sudhakar, C., (Eds.), Infection Control - Updates. InTech, 2012, p. 93-114.

Wentzel, M.J., Beerlage-de Jong, N., Sieverink, F. \& Van Gemert-Piinen, J.E.W.C., Slimmer eHealth ontwikkelen en implementeren met de CeHRes Roadmap. In: Politiek, C., \& Hoogendijk, R., (Eds.), Co-creatie eHealthboek; eHealth, technisch kunstje of pure veranderkunde?, 2014, p. 242-250.

Journal articles [not peer-reviewed]

De Jong, N., \& Van Gemert-Pijnen, J.E.W.C., Gebruikersgerichte basishygiëneprotocollen: de toekomst voor veilige zorg. Infectieziekten bulletin, 2013, $8(24)$, p. $258-262$.

De Jong, N., Wentzel, M.J. and Gemert-Pijnen, J.E.W.C. van (2012) Patiëntenparticipatie: Wat moeten burgers weten over MRSA en antibioticagebruik? Infectieziekten bulletin, 23 (10), p. 343 - 348.

Wentzel, M.J., De Jong, N., Grensoverschrijdende aanpak infectieziekten hard nodig, Infectieziekten Platform, 2012. 


\section{Conference proceedings}

Wentzel, M.J., Beerlage-de Jong, N. \& Van Der Geest, Th.M., Redesign Based on Card Sorting: How Universally Applicable are Card Sort Results? In: Duffy, V., (Eds.), Digital Human Modeling: Applications in Health, Safety, Ergonomics and Risk Management, Springer, 2016, p. 381-388.

De Jong, N., Wentzel, M.J., Kelders, S., Oinas-Kukkonen, H. \& Van Gemert-Piinen, J.E.W.C., Evaluation of perceived persuasiveness constructs by combining user tests and expert assessments. In: Öörni, A., Kelders, S.M., Van Gemert-Piinen, J.E.W.C. \& OinasKukkonen, H., (Eds.), BCSS 2014: Behavior Change Support Systems, 2014, p. 7-15.

De Jong, N., Eikelenboom-Boskamp, A., Voss, A. \& Van Gemert-Piinen, J.E.W.C., Usercentered and persuasive design of a web-based registration and monitoring system for healthcare-associated infections in nursing homes. In: Van Gemert-Piinen, J.E.W.C., Hettinga, M.,\& Smedberg, A., (Eds.), The Sixth International Conference on eHealth, Telemedicine, and Social Medicine (eTELEMED 2014), IARIA, 2014, p. 152-157.

\section{Poster presentations}

Beerlage-de Jong, N., Kulyk, O., Wentzel, M.J., Oinas-Kukkonen, H. \& Van GemertPijnen, J.E.W.C., 'Sorting out' the PPQ: A mixed-methods approach to evaluate Perceived Persuasiveness Questionnaire constructs. The 10th International Conference on Persuasive Technology (PERSUASIVE 2015). Chicago, Illinois, USA (June 3-5), 2015.

De Jong, N., Eikelenboom-Boskamp, A., Voss, A. \& Van Gemert-Pijnen, J.E.W.C., Improving patient safety via a persuasive registration and monitoring system for infections in nursing homes. International Congress of Behavioral Medicine: Groningen, the Netherlands (August 20-23), 2014.

De Jong, N., Wentzel, M.J., Van Velsen, L., \& Van Gemert-Pijnen, J.E.W.C., From problem to eHealth: Benefits of early stage user-involvement. Medicine 2.0: London, UK (September 22-24), 2013.

\section{Other output}

Infection manager http://beta.infectionmanager.com

Prevalence app http://vps42232.public.cloudvps.com/432/prevalentie-metingverpleeghuizen/ 
188 | It always seems impossible, until it is done - Nelson Mandela 


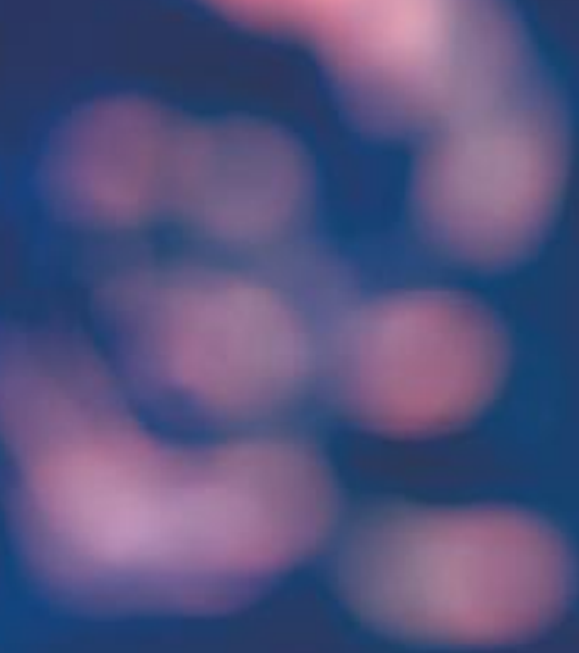

Nienke Beerlage-de Jong is a postdoctorat researcher at the Univereity of Twente and Univereity Medical Centre Groningen. Her PhD research was performed for the department of Peychology. Heatth \& Technology, at the Centre for eHealth and Wellbeing Reeserch of the Univereity of Twente, It was performed in the INTERREG IVa EurBafety Heatth-net project, which is aimed to improve patient safety in crose-border healthcare.

Her main research intereste include the participatory development. pereuasive design and evaluation of eHealth technology. 